Florida International University FIU Digital Commons

$11-14-2014$

\title{
Improved Criteria for Estimating Calibration Factors for Highway Safety Manual (HSM) Applications
}

Dibakar Saha

Florida International University, dsaha003@fiu.edu

DOI: $10.25148 /$ etd.FI14110774

Follow this and additional works at: https://digitalcommons.fiu.edu/etd

Part of the Civil and Environmental Engineering Commons

\section{Recommended Citation}

Saha, Dibakar, "Improved Criteria for Estimating Calibration Factors for Highway Safety Manual (HSM) Applications" (2014). FIU Electronic Theses and Dissertations. 1701.

https://digitalcommons.fiu.edu/etd/1701 


\section{FLORIDA INTERNATIONAL UNIVERSITY}

Miami, Florida

\section{IMPROVED CRITERIA FOR ESTIMATING CALIBRATION FACTORS FOR HIGHWAY SAFETY MANUAL (HSM) APPLICATIONS}

A dissertation submitted in partial fulfillment of

the requirements for the degree of

DOCTOR OF PHILOSOPHY

in

CIVIL ENGINEERING

by

Dibakar Saha

2014 
To: Dean Amir Mirmiran

College of Engineering and Computing

This dissertation, written by Dibakar Saha, and entitled Improved Criteria for Estimating Calibration Factors for Highway Safety Manual (HSM) Applications, having been approved in respect to style and intellectual content, is referred to you for judgment.

We have read this dissertation and recommend that it be approved.

Fabian Cevallos

Zhenmin Chen

Mohammed Hadi

L. David Shen

Priyanka Alluri

Albert Gan, Major Professor

Date of Defense: November 14, 2014

The dissertation of Dibakar Saha is approved.

$\begin{array}{r}\text { Dean Amir Mirmiran } \\ \text { College of Engineering and Computing } \\ \hline \begin{array}{r}\text { Dean Lakshmi N. Reddi } \\ \text { University Graduate School }\end{array}\end{array}$

Florida International University, 2014 
(C) Copyright 2014 by Dibakar Saha

All rights reserved. 


\section{DEDICATION}

I dedicate this dissertation to my parents, Dipak Kumar Saha and Suvra Saha, and to my wife, Tamalika Saha (Tithi), for their unconditional love and support. 


\section{ACKNOWLEDGMENTS}

I would like to take this opportunity to acknowledge everyone who helped me to reach my dream of achieving the doctoral degree. First and foremost, I would like to express my gratitude to my advisor and mentor, Dr. Albert Gan, for his continuous guidance, constructive criticism, and directive suggestions at every stage of the dissertation. I am thankful to him not only for his thoughtful insights provided in completing the dissertation, but also for his care in every other aspect during the time of my doctoral study at Florida International University. I consider myself fortunate enough to have been one of the students of Dr. Gan. I will be grateful to him throughout my life.

I am also grateful to Dr. Priyanka Alluri for giving me the privilege to work on her research project. She had been watchful from the very beginning of the research and guided me with critical problem-solving ideas till the completion of the processes. Without Dr. Alluri's consistent instructions in this research, the dissertation would become impossible. I am also thankful to Dr. Alluri for her rounds of edits and corrections in preparing the dissertation.

I would also like to thank all my other committee members, Dr. L. David Shen, Dr. Mohammed Hadi, Dr. Zhenmin Chen, and Dr. Fabian Cevallos for their interest in my research. I sincerely appreciate their invaluable time for reading the dissertation and providing comments and suggestions to improve it.

I am also thankful to Dr. Kaiyu Liu for developing and managing a data collection system for this research. No appreciation is enough for the efforts taken by the following graduate assistants of the Transportation Information Research Group to collect the data: Ms. Homa Fartash, Ms. Maria Guevara, Mr. Mohammad Lavasani, Ms. Katrina Meneses, 
Ms. Stephanie Miranda, Ms. Anita Pourji, Mr. Asif Raihan, and Mr. Eazaz Sadeghvaziri. I would also like to thank the following colleagues for collaborating with me and helping me pass a good time all of these years: Dr. Kirolos Haleem, Dr. Wanyang Wu, Dr. Jinyan Lu, Mr. Li Tang, and Ms. Nishat Zaman. Furthermore, I would like to acknowledge Florida Department of Transportation Research Center for providing financial support for this research.

I would like to convey a very special thanks to all my family members for their boundless love, support, and encouragement in pursuing my doctoral degree. 


\section{ABSTRACT OF THE DISSERTATION \\ IMPROVED CRITERIA FOR ESTIMATING CALIBRATION FACTORS FOR HIGHWAY SAFETY MANUAL (HSM) APPLICATIONS}

by

Dibakar Saha

Florida International University, 2014

Miami, Florida

\section{Professor Albert Gan, Major Professor}

The Highway Safety Manual (HSM) estimates roadway safety performance based on predictive models that were calibrated using national data. Calibration factors are then used to adjust these predictive models to local conditions for local applications. The HSM recommends that local calibration factors be estimated using 30 to 50 randomly selected sites that experienced at least a total of 100 crashes per year. It also recommends that the factors be updated every two to three years, preferably on an annual basis. However, these recommendations are primarily based on expert opinions rather than data-driven research findings. Furthermore, most agencies do not have data for many of the input variables recommended in the HSM. This dissertation is aimed at determining the best way to meet three major data needs affecting the estimation of calibration factors: (1) the required minimum sample sizes for different roadway facilities, (2) the required frequency for calibration factor updates, and (3) the influential variables affecting calibration factors.

In this dissertation, statewide segment and intersection data were first collected for most of the HSM recommended calibration variables using a Google Maps 
application. In addition, eight years (2005-2012) of traffic and crash data were retrieved from existing databases from the Florida Department of Transportation. With these data, the effect of sample size criterion on calibration factor estimates was first studied using a sensitivity analysis. The results showed that the minimum sample sizes not only vary across different roadway facilities, but they are also significantly higher than those recommended in the HSM. In addition, results from paired sample t-tests showed that calibration factors in Florida need to be updated annually.

To identify influential variables affecting the calibration factors for roadway segments, the variables were prioritized by combining the results from three different methods: negative binomial regression, random forests, and boosted regression trees. Only a few variables were found to explain most of the variation in the crash data. Traffic volume was consistently found to be the most influential. In addition, roadside object density, major and minor commercial driveway densities, and minor residential driveway density were also identified as influential variables. 


\section{TABLE OF CONTENTS}

CHAPTER

PAGE

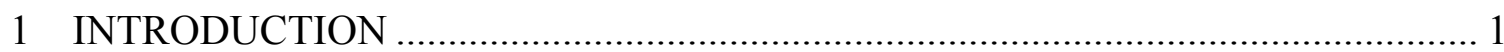

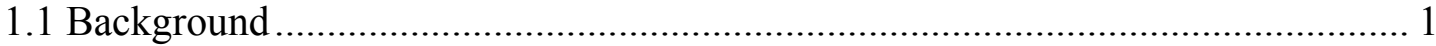

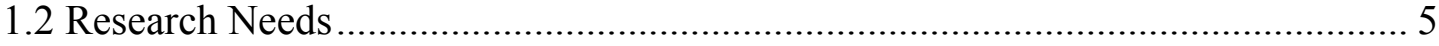

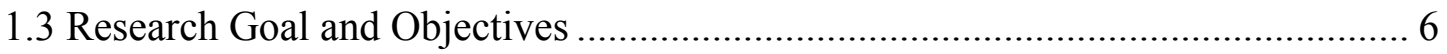

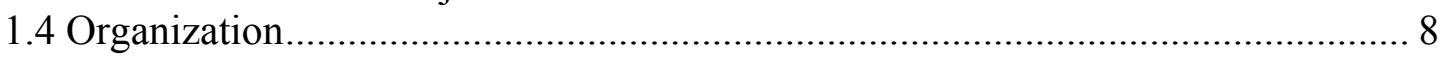

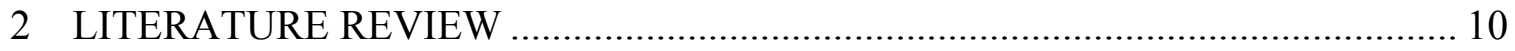

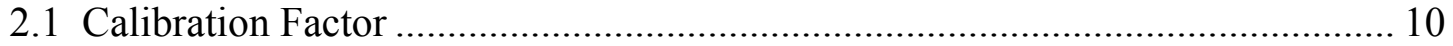

2.1.1 Need for Calibration Factor ................................................................... 10

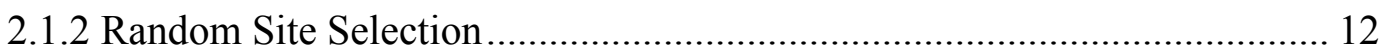

2.1.3 Sampling in States' HSM Calibration....................................................... 14

2.1.4 Evaluation of Calibration Sample Size Criteria.......................................... 23

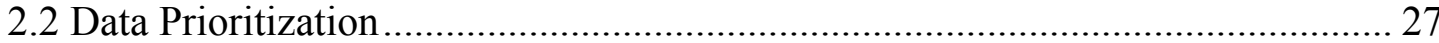

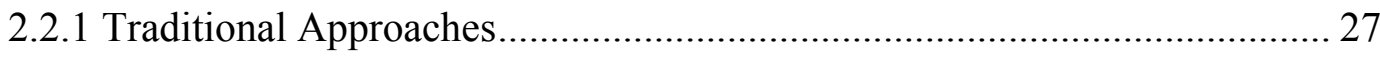

2.2.2 Data Mining Approaches …………….................................................. 30

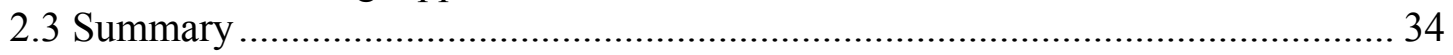

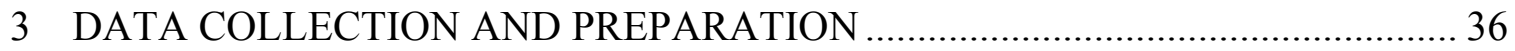

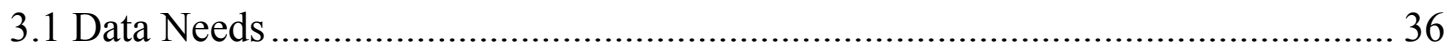

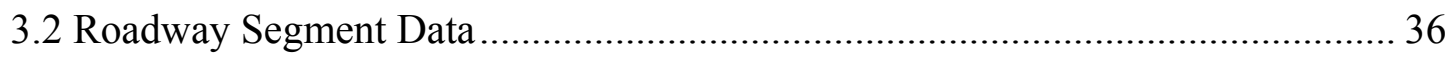

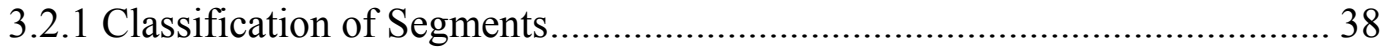

3.2.2 Generation of Homogeneous Segments ................................................... 42

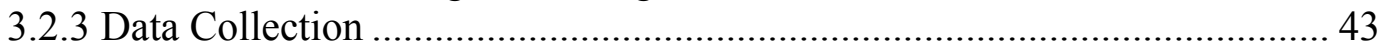

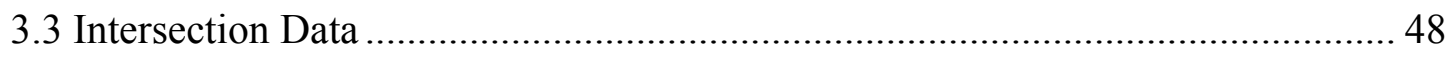

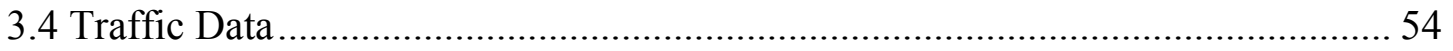

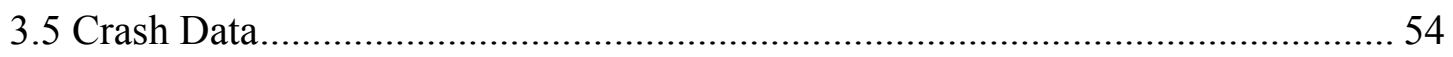

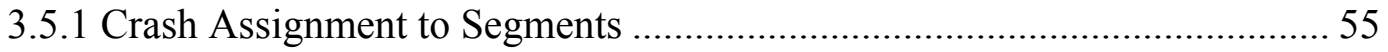

3.5.2 Crash Assignment to Intersections............................................................. 56

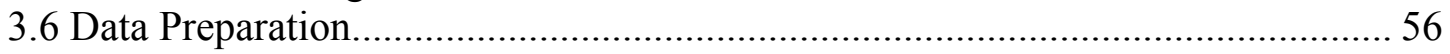

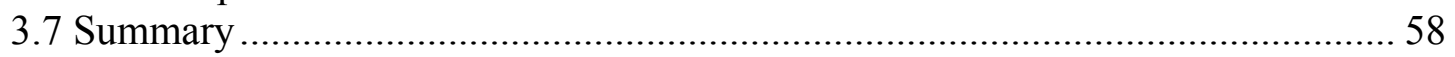

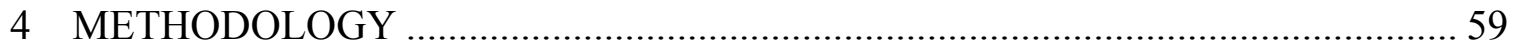

4.1 Methodological Framework......................................................................... 59

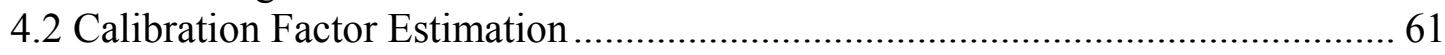

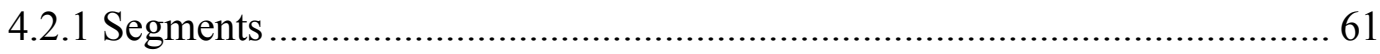

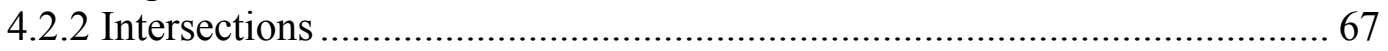

4.3 Sensitivity Analysis of Calibration Sample Size ……………………………..... 74

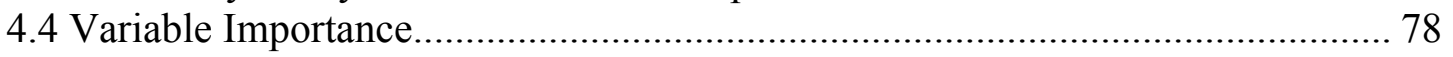

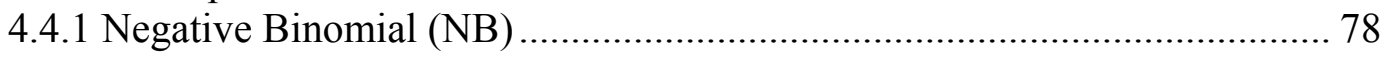

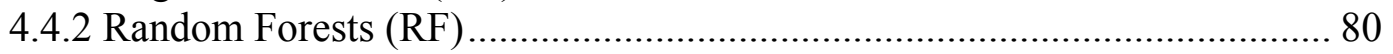

4.4.3 Boosted Regression Trees (BRT) …………….................................... 81 


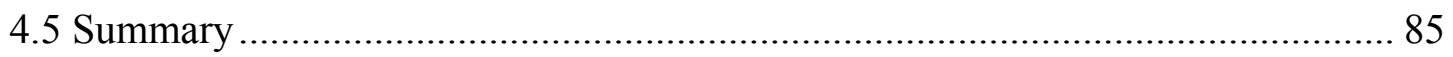

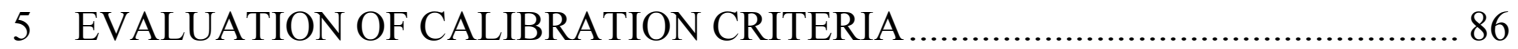

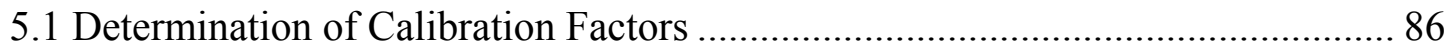

5.2 Effect of Sample Size on Calibration Factors.................................................... 89

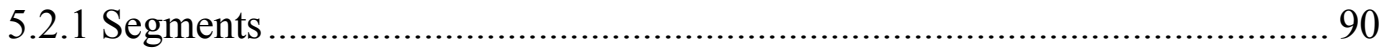

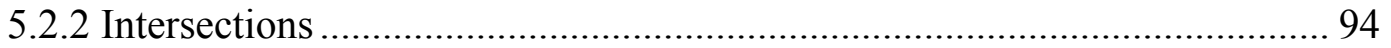

5.3 Recommendations on Minimum Sample Size ................................................... 94

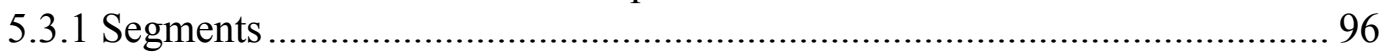

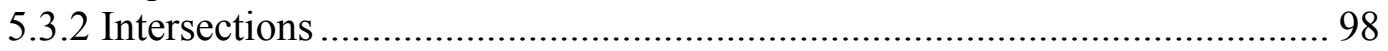

5.4 Validation of the Recommended Frequency for Calibration Factor Updates..... 100

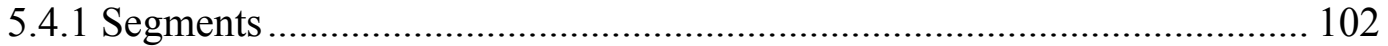

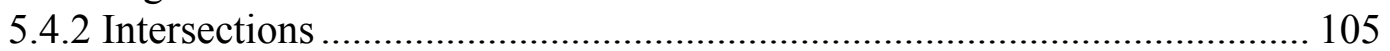

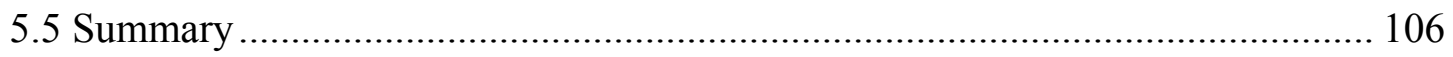

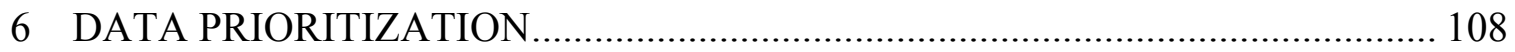

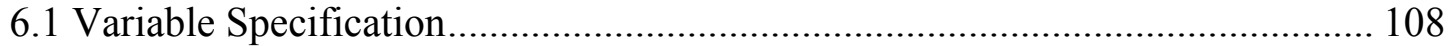

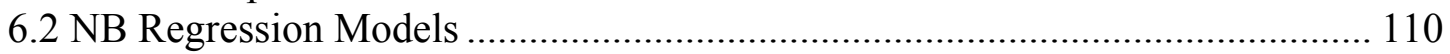

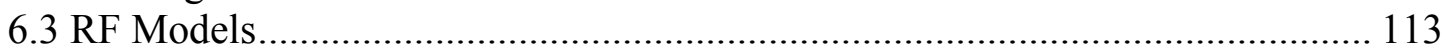

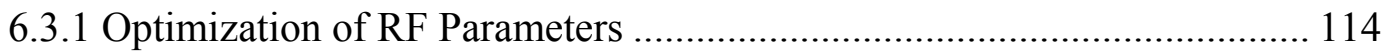

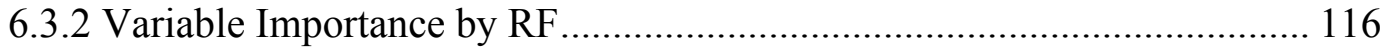

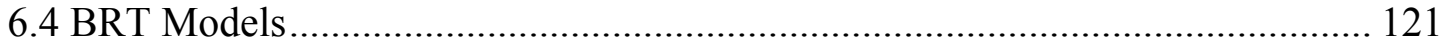

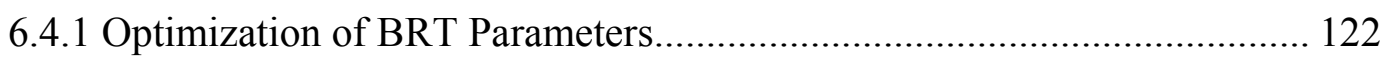

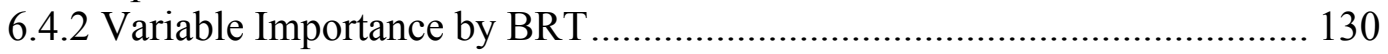

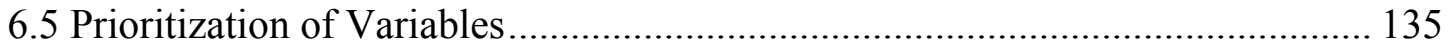

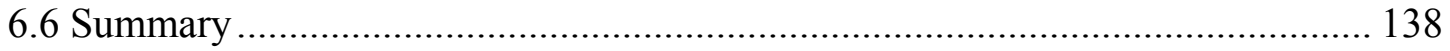

7 CONCLUSIONS AND RECOMMENDATIONS ................................................ 140

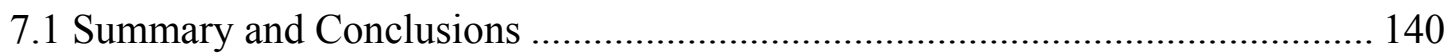

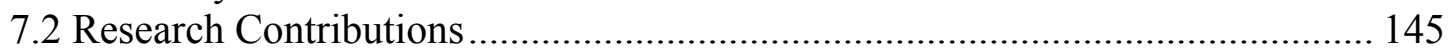

7.3 Recommendations for Future Research ........................................................... 146

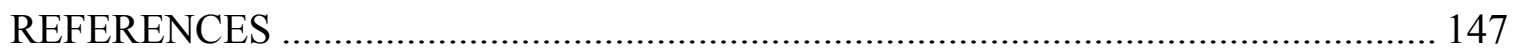

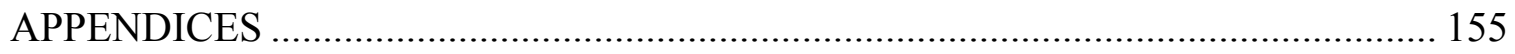

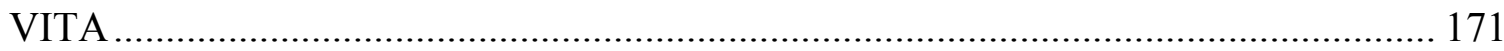




\section{LIST OF TABLES}

TABLE

PAGE

1-1 Segment and Intersection Types Covered in the HSM Predictive Method................. 2

1-2 Roadway Characteristics Data Requirements for Part C of the HSM......................... 7

2-1 Summary of Results Showing the Effect of Site Selection Types (Sun et al., 2006).13

2-2 Sample Sizes Used in Calibrating the HSM Prediction Models 14

3-1 Data Variables Identified in the HSM for Urban and Suburban Arterials

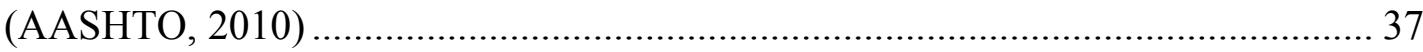

3-2 Summary Statistics of Urban and Suburban Arterial Segments................................ 57

3-3 Summary Statistics of Urban and Suburban Intersections ……………………….... 57

5-1 Calibration Factors Computed Using Single Year of Data for Urban and Suburban

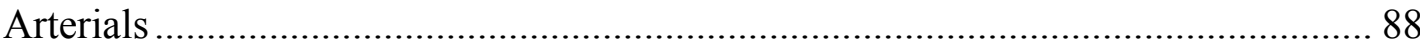

5-2 Calibration Factors Computed Using Three Years of Data for Urban and Suburban

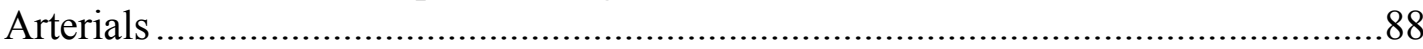

5-3 Sensitivity Analysis Results for Urban and Suburban Arterial Segments Using

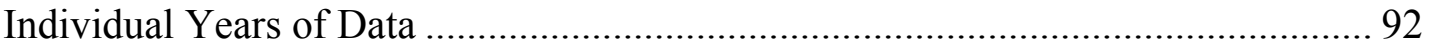

5-4 Sensitivity Analysis Results for Urban and Suburban Arterial Segments Using Three Consecutive Years of Data.......................................................................... 93

5-5 Sensitivity Analysis Results for Urban and Suburban Intersections Using Individual Years of Data

5-6 Sensitivity Analysis Results for Urban and Suburban Intersections Using Three

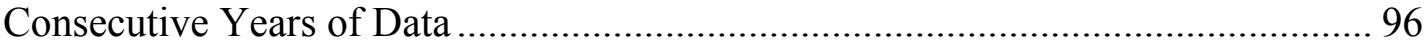

5-7 Paired t-Test Results for Urban and Suburban Arterial Segments for Scenario 1 .. 103

5-8 Paired t-Test Results for Urban and Suburban Arterial Segments for Scenario 2 .. 103

5-9 Paired t-Test Results for Urban and Suburban Arterial Segments for Scenario 3 .. 104

5-10 Paired t-Test Results for Urban and Suburban Intersections for Scenario 1 ................. 105

5-11 Paired t-Test Results for Urban and Suburban Intersections for Scenario 2 2................. 106

5-12 Paired t-Test Results for Urban and Suburban Intersections for Scenario 3.................. 106 
5-13 Recommended Sample Size for Urban and Suburban Arterial Segments. 107

5-14 Recommended Sample Size for Urban and Suburban Intersections ... 107

6-1 Variables in the HSM for Urban and Suburban Arterial Segments 109

6-2 NB Model Results for Urban and Suburban Two-Lane Undivided Arterials

6-3 NB Model Results for Urban and Suburban Three-Lane Arterials with a TWLTL 111

6-4 NB Model Results for Urban and Suburban Four-Lane Undivided Arterials 112

6-5 NB Model Results for Urban and Suburban Four-Lane Divided Arterials. 112

6-6 NB Model Results for Urban and Suburban Five-Lane Arterials with a TWLTL.. 113

6-7 Performance of RF Models Developed for Urban and Suburban Arterial Segments

6-8 Variable Importance by RF Model for Urban and Suburban Two-Lane Undivided Arterials

6-9 Variable Importance by RF Model for Urban and Suburban Three-Lane Arterials with a TWLTL.

6-10 Variable Importance by RF Model for Urban and Suburban Four-Lane Undivided Arterials ....

6-11 Variable Importance by RF Model for Urban and Suburban Four-Lane Divided Arterials

6-12 Variable Importance by RF Model for Urban and Suburban Five-Lane Arterials with a TWLTL

6-13 Minimum Poisson Deviance of BRT models for Urban and Suburban Arterial Segments 130

6-14 Variable Importance by BRT Model for Urban and Suburban Two-Lane Undivided Arterials .

6-15 Variable Importance by BRT Model for Urban and Suburban Three-Lane Arterials with a TWLTL

6-16 Variable Importance by BRT Model for Urban and Suburban Four-Lane Undivided Arterials .

6-17 Variable Importance by BRT Model for Urban and Suburban Four-Lane Divided Arterials 134 
6-18 Variable Importance by BRT Model for Urban and Suburban Five-Lane Arterials with a TWLTL

6-19 Variable Importance by NB, RF, and BRT Models for Urban and Suburban Two-Lane Undivided Arterials

6-20 Prioritization of Variables

A-1 Descriptive Statistics for Urban and Suburban Two-lane Undivided Arterials (Number of Segments $=1,791$ )

A-2 Descriptive Statistics for Urban and Suburban Three-lane Arterials with a TWLTL (Number of Segments $=359$ ).

A-3 Descriptive Statistics for Urban and Suburban Four-lane Undivided Arterials (Number of Segments $=266$ )

A-4 Descriptive Statistics for Urban and Suburban Four-lane Divided Arterials (Number of Segments $=4,969$ )

A-5 Descriptive Statistics for Urban and Suburban Five-lane Arterials with a TWLTL (Number of Segments $=1,084$ )

A-6 Descriptive Statistics for Urban and Suburban Three-Leg Stop-Controlled Intersections (Number of Intersections $=317$ )

A-7 Descriptive Statistics for Urban and Suburban Four-Leg Signalized Intersections (Number of Intersections $=397$ )

B-1 Coefficients of SPF for Multiple-Vehicle Non-Driveway Crashes on Urban and Suburban Arterial Segments

B-2 Coefficients of SPF for Single-Vehicle Crashes on Urban and Suburban Arterial Segments

B-3 Coefficients of SPF for Multiple-Vehicle Driveway-Related Crashes on Urban and Suburban Arterial Segments

B-4 On-Street Parking Factor $\left(f_{p k}\right)$ Used in Determining CMF for On-Street Parking.. 166

B-5 Fixed-Object Offset Factor $\left(f_{\text {offset }}\right)$ Used in Determining CMF for Roadside Fixed Objects

B-6 CMFs for Median Width on Urban and Suburban Four-Lane Divided Arterials ... 166

B-7 Coefficients of SPF for Multiple-Vehicle Crashes on Urban and Suburban Intersections 
B-8 Coefficients of SPF for Single-Vehicle Crashes on Urban and Suburban

Intersections.

B-9 Coefficients of SPF for Vehicle-Pedestrian Crashes on Urban and Suburban Intersections.....

C-1 Proportion of Total Crashes That Occurred at Night on Unlighted Segments $\left(p_{n r}\right)$ of Urban and Suburban Arterials.

C-2 Proportion of Total Nighttime Crashes That Involved a Fatality or Injury on Unlighted Segments $\left(p_{i n r}\right)$ of Urban and Suburban Arterials 169

C-3 Proportion of Total Nighttime Crashes That Involved PDO on Unlighted Segments $\left(p_{p n r}\right)$ of Urban and Suburban Arterials

C-4 Proportion of Total Crashes That Occurred at Night on Unlighted Intersections $\left(P_{n i}\right)$ on Urban and Suburban Arterials.

C-5 Bicycle Crash Adjustment Factors for Intersections $\left(f_{\text {bikei }}\right)$ on Urban and Suburban Arterials.

C-6 Pedestrian Crash Adjustment Factors for Intersections $\left(f_{\text {bikei }}\right)$ on Urban and Suburban Arterials

C-7 Proportion of Specific Crashes on Urban and Suburban Four-Leg Signalized Intersections 


\section{LIST OF FIGURES}

FIGURE

PAGE

2-1 Illustration of a Decision Tree (Kashani and Mohaymany, 2011) ........................... 31

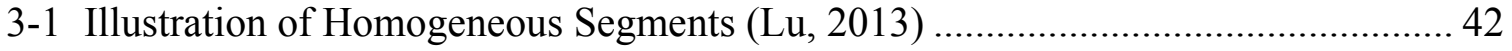

3-2 VRICS Application for Segment Data Collection ............................................ 45

3-3 VRICS Application for Intersection Data Collection......................................... 50

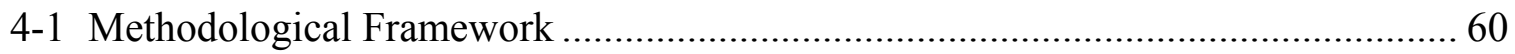

6-1 Performance of BRT Models for Urban and Suburban Two-Lane Undivided Arterials

6-2 Performance of BRT Models for Urban and Suburban Three-Lane Arterials with a TWLTL

6-3 Performance of BRT Models for Urban and Suburban Four-Lane Undivided Arterials

6-4 Performance of BRT Models for Urban and Suburban Four-Lane Divided Arterials

6-5 Performance of BRT Models for Urban and Suburban Five-Lane Arterials with a TWLTL 


\section{ABBREVIATIONS AND ACRONYMS}

\begin{tabular}{ll} 
AADT & Annual Average Daily Traffic \\
AASHTO & American Association of State Highway and Transportation Officials \\
ADT & Average Daily Traffic \\
ANOVA & Analysis of Variance \\
BRT & Boosted Regression Trees \\
CART & Classification and Regression Trees \\
CMF & Crash Modification Factor \\
CURE & Cumulative Residual \\
CV & Cross Validation \\
DOF & Degree of Freedom \\
EB & Empirical Bayes \\
FDOT & Florida Department of Transportation \\
FHWA & Federal Highway Administration \\
FI & Fatal and Injury \\
GIS & Geographic Information Systems \\
GLM & Generalized Linear Model \\
HCL & High Crash Location \\
HSM & Highway Safety Manual \\
HTBR & Hierarchical Tree-Based Regression \\
LaDOTD & Louisiana Department of Transportation and Development \\
MoDOT & Missouri Department of Transportation \\
MPB & Mean Prediction Bias \\
\hline
\end{tabular}




\begin{tabular}{|c|c|}
\hline MSE & Mean Squared Error \\
\hline MSPE & Mean Squared Prediction Error \\
\hline NB & Negative Binomial \\
\hline OOB & Out-of-Bag \\
\hline PDO & Property Damage Only \\
\hline PSI & Potential for Safety Improvement \\
\hline RCI & Roadway Characteristics Inventory \\
\hline RDWTBL & Roadway Table \\
\hline RF & Random Forests \\
\hline RTM & Regression-to-the-Mean \\
\hline RTOR & Right Turn On Red \\
\hline SHS & State Highway System \\
\hline SPF & Safety Performance Function \\
\hline SQL & Structured Query Language \\
\hline TWLTL & Two-Way Left-Turn Lane \\
\hline UBR & Unified Basemap Repository \\
\hline VDOT & Virginia Department of Transportation \\
\hline VRICS & Visual Roadway Inventory Collection System \\
\hline
\end{tabular}




\section{CHAPTER 1 \\ INTRODUCTION}

\subsection{Background}

The Highway Safety Manual (HSM), published by the American Association of State Highway and Transportation Officials (AASHTO) in 2010, is designed "to assist agencies in their effort to integrate safety into their decision-making processes" (AASHTO, 2010). The HSM provides methods to perform all the following six steps in the road safety management process: (1) network screening, (2) diagnosis, (3) countermeasure selection, (4) economic appraisal, (5) project prioritization, and (6) safety effectiveness evaluation. Of all these steps, network screening is considered the most critical step which involves reviewing a transportation road network, identifying high crash locations (HCLs), and prioritizing the locations based on their potential for safety improvement (PSI). The HSM recommends using the empirical Bayes (EB) method to screen locations for safety improvements. The EB method accounts for the effect of the traditional regression-to-the-mean (RTM) bias. The RTM bias occurs when sites for safety improvements are selected based on short-term observed crash frequency; thus, reducing the cost-effectiveness of safety programs. The EB method requires the use of predictive models that estimate the predicted average crash frequency of a site, facility, or network.

Part $\mathrm{C}$ of the HSM presents predictive models to estimate the average predicted crash frequencies for different roadway facilities, including rural two-lane two-way roads, rural multilane highways, and urban and suburban arterials. Table 1-1 lists the segment and intersection types discussed under each facility in the HSM. Although the 
models vary by each facility and site type, the general form of the predictive models can be expressed as follows:

$$
N_{\text {predicted }, x}=N_{s p f, x} \times\left(C M F_{1, x} \times C M F_{2, x} \times \ldots \times C M F_{n, x}\right) \times C_{x}
$$

where,

$$
\begin{aligned}
N_{\text {predicted }, i}= & \text { predicted average crash frequency for a specific year on site type } x, \\
N_{s p f, i}= & \text { predicted average crash frequency for a specific year for site type } x \\
& \text { for base conditions, } \\
C M F_{n, x}= & \text { crash modification factors for } n \text { geometric conditions or traffic } \\
& \text { control features for site type } x, \text { and } \\
= & \text { calibration factor to adjust for local conditions for site type } x .
\end{aligned}
$$

\begin{tabular}{|c|c|c|}
\hline $\begin{array}{l}\text { Rural Two-Lane, Two-Way } \\
\text { Roads }\end{array}$ & Rural Multilane Highways & Urban and Suburban Arterials \\
\hline \multicolumn{3}{|c|}{ Segments } \\
\hline - Two-lane undivided & $\begin{array}{l}\text { - Four-lane undivided } \\
\text { - Four-lane divided }\end{array}$ & $\begin{array}{l}\text { - Two-lane undivided } \\
\text { - Three-lane with a two-way } \\
\text { left-turn lane (TWLTL) } \\
\text { - Four-lane undivided } \\
\text { - Four-lane divided } \\
\text { - Five-lane with a TWLTL }\end{array}$ \\
\hline \multicolumn{3}{|c|}{ Intersections } \\
\hline $\begin{array}{l}\text { - Three-leg stop-controlled } \\
\text { - Four-leg stop-controlled } \\
\text { - Four-leg signalized }\end{array}$ & $\begin{array}{l}\text { - Three-leg stop-controlled } \\
\text { - Four-leg stop-controlled } \\
\text { - Four-leg signalized }\end{array}$ & $\begin{array}{l}\text { - Three-leg stop-controlled } \\
\text { - Four-leg stop-controlled } \\
\text { - Three-leg signalized } \\
\text { - Four-leg signalized }\end{array}$ \\
\hline
\end{tabular}

TABLE 1-1 Segment and Intersection Types Covered in the HSM Predictive Method

As can be observed from Equation (1-1), the three key components required to estimate predicted average crash frequency are:

- base safety performance functions (SPFs),

- crash modification factors (CMFs), and 
- calibration factor.

The base SPF is a statistical regression model that establishes a relationship between crash occurrence and the associated factors under specific base conditions. The base conditions in the HSM usually correspond to given geometric characteristics, roadway environment, and traffic control features of sites. For example, the base conditions for rural two-lane roadway segments are lane width of $12 \mathrm{ft}$ lanes, $6 \mathrm{ft}$ paved shoulder, roadside hazard rating of 3 , five driveways per mile, absence of horizontal and vertical curve, absence of centerline rumble strips, absence of passing lane, absence of two-way left-turn lane (TWLTL), absence of street lighting, and absence of automated speed enforcement. The base SPFs in the HSM estimate the predicted average crash frequency as a function of average annual daily traffic (AADT) and segment length for roadway segments, and AADT on major road and AADT on minor road approaches for intersections. Mathematically, the base SPF for segments can be expressed as:

$$
N_{s p f r s}=e^{\alpha_{0}} \times A A D T^{\alpha_{1}} \times L
$$

where,

$$
\begin{aligned}
N_{s p f r s}= & \text { predicted average crash frequency per year for a roadway segment with } \\
& \text { base conditions, } \\
A A D T= & \text { average annual daily traffic (vehicles per day) of a roadway segment, } \\
L \quad= & \text { segment length (miles) } \\
\alpha_{0} \quad= & \text { intercept of the model, and } \\
\alpha_{1} \quad= & \text { coefficient of AADT. }
\end{aligned}
$$

Similarly, the base SPF for intersections is expressed as: 


$$
N_{\text {spf int }}=e^{\beta_{0}} \times A A D T_{\text {major }}^{\beta_{1}} \times A A D T_{\text {minor }}^{\beta_{2}}
$$

where,

$$
\begin{aligned}
& N_{\text {spf int }}=\text { predicted average crash frequency per year for an intersection with } \\
& \text { base conditions, } \\
& A A D T_{\text {major }}=\text { average annual daily traffic at the major road of an intersection } \\
& \text { (vehicles per day), } \\
& A A D T_{\text {minor }}=\text { average annual daily traffic at the minor road of an intersection } \\
& \text { (vehicles per day), } \\
& \beta_{0} \quad=\text { intercept of the model, and } \\
& \beta_{1}, \beta_{2}=\text { coefficients of } A A D T_{\text {major }} \text { and } A A D T_{\text {minor }} \text {, respectively. }
\end{aligned}
$$

In cases when sites deviate from the pre-defined base conditions, crash modification factors (CMFs) are multiplied with the predicted crash frequency calculated using the base SPFs to account for the effects of non-base conditions on predicted crashes. The CMFs are calculated as the ratio of the effectiveness of one condition to that of another condition.

The coefficients of the base SPFs and the values of the CMFs in the HSM were developed based on data collected from few states: California, Michigan, Minnesota, New York State, Texas, and Washington State. Therefore, the HSM recommends for calibration of the default models "to account for differences between the jurisdiction and time period for which the predictive models were developed and the jurisdiction and time period to which they are applied by HSM users" (AASHTO, 2010). The differences between jurisdictions are reflected in factors related to geographic area, weather, roadway 
environment, drivers' characteristics, vulnerable road users, animal inhabitants, and crash reporting thresholds. Calibration factor is estimated as the ratio of the total number of observed crashes to the total number of predicted crashes calculated using the SPFs and CMFs provided in the HSM. The predictive models are most effective when calibrated to local conditions (Findley et al., 2012; Lu, 2013; Sun et al., 2006; and Young and Park, 2013).

\subsection{Research Needs}

The HSM outlines the following criteria for deriving calibration factors:

- a minimum of 30 to 50 sites should be selected through random sampling,

- selected sites should experience at least 100 crashes per year, and

- calibration factors be updated at least every two to three years.

These recommendations are primarily based on expert opinions rather than datadriven research findings. Given that roadway characteristics of different roadway types are likely to have different levels of homogeneity, the recommended sample size of 30 to 50 sites could be too many for some roadway types, yet too few for others. Also, the criterion of a minimum of 100 annual crashes is debatable given the fact that the number of crashes vary widely across different roadway types. For example, intersections would generally experience many more crashes than mid-block segments. Similarly, urban arterials usually experience many more crashes than rural counterparts. Therefore, the recommendation regarding sample size to produce a more reliable and accurate calibration factor needs to be verified.

Since the calibration factor estimation requires extensive efforts and roadway geometric features do not change substantially from year to year, it might not be 
necessary to update calibration factors frequently, say, annually or every two to three years. Specific guidance on how frequently calibration factors needs to be updated by validating the intervals recommended in the HSM would be helpful for the agencies.

Again, the calibration process involves the use of very detailed information regarding roadway geometry, traffic, and intersection characteristics to accurately represent the agency's safety performance (see Table 1-2). However, several variables are often unavailable in most of the states' roadway inventory databases. For example, many of these variables are currently unavailable in Florida's roadway characteristics inventory (RCI) database. Also, the process of collecting and maintaining all the data variables for the entire road network for the purpose of implementing the manual is not cost-feasible. Therefore, a process to streamline the data requirements that minimizes the potential impacts to the quality of analysis is quite desirable. Given that not all of the variables are likely to have the same impact on safety predictions, it becomes beneficial to assess and rank the impact of each variable on crash predictions. The ranking will help prioritize the data collection needs such that the benefit is greatest.

\subsection{Research Goal and Objectives}

The goal of this dissertation research is to determine the best way to meet the data needs affecting the calibration factor estimations for urban and suburban arterials on Florida's state highway system. Accordingly, the specific objectives of this dissertation are to:

1. evaluate the effect of sample size on the calibration factor estimates and determine minimum sample sizes, 
2. validate the recommended calibration factor update frequency, and

3. determine the influence of variables on crash predictions and to identify and prioritize the influential calibration variables.

TABLE 1-2 Roadway Characteristics Data Requirements for Part C of the HSM (AASHTO, 2010)

\begin{tabular}{|l|l|}
\hline Roadway Segment Characteristics Data & Intersection Characteristics Data \\
\hline - Area type & - Area type \\
- Functional class & - Number of lanes \\
- Number of lanes & - Average annual daily traffic (AADT) for \\
- Segment length & major road \\
- Average Annual Daily Traffic (AADT) & - AADT for minor road \\
- Lane width & - Number of legs \\
- Shoulder type and width & - Traffic control type \\
- Presence of center two-way left-turn lane & - Intersection skew angle \\
- Median width & - Number of approaches with left-turn \\
- Side-slope & lanes \\
- Length, and radius of horizontal curves & - Number of approaches with right-turn \\
- Length of tangents & lanes \\
- Presence of spiral transition for horizontal & - Presence of lighting \\
curves & - Presence of right-turn-on-red (RTOR) \\
- Superelevation variance for horizontal & signal operation \\
curves & - Presence and type of left-turn signal \\
- Vertical grade & phasing \\
- Presence of lighting & - Presence of red-light cameras \\
- Driveway density & - Number of bus stops within $1,000 \mathrm{ft}$ of \\
- Type of land-use & intersection \\
- Speed limit & - Presence of schools within 1,000 ft of \\
- Presence and type of on-street parking & intersection \\
- Curb length with on-street parking & - Number of alcohol sales establishments \\
- Presence of passing lane & within 1,000 ft of intersection \\
- Presence of short-four lane section & - Pedestrian activity level \\
- Presence of centerline rumble strip & - Maximum number of lanes crossed by \\
- Roadside hazard rating (RHR) & pedestrians \\
- Number of roadside fixed objects & \\
- Average offset to roadside fixed objects & \\
- Presence of automated speed enforcement & \\
\hline
\end{tabular}


This research focuses only on urban and suburban arterial roadway segments and intersections on state roads in Florida. As such, the scope of this research is limited to the following roadway segment and intersection types:

- urban and suburban two-lane undivided arterials,

- urban and suburban three-lane arterials with a center two-way left-turn lane (TWLTL),

- urban and suburban four-lane undivided arterials,

- urban and suburban four-lane divided arterials,

- urban and suburban five-lane arterials with a center TWLTL,

- urban and suburban three-leg intersections with stop control at the minor road, and

- urban and suburban four-leg signalized intersections.

\subsection{Organization}

The organization of the rest of the dissertation is as follows. Chapter 2 presents a comprehensive review of the studies that developed calibration factors for implementing the HSM. It also provides a review of the existing literature on computing variable importance. Chapter 3 describes the detailed data collection and preparation efforts undertaken in this dissertation. Chapter 4 discusses the methodology followed in this dissertation to accomplish the research objectives. Chapters 5 and 6 present the research results. Specifically, Chapter 5 discusses the effect of sample sizes on calibration factors for urban and suburban arterials in Florida, It also recommends the minimum sample size required to estimate reliable calibration factors. Chapter 6 focuses on the generation and application of different models for variable prioritization. It also provides the prioritized list of HSM calibration variables. Chapter 7 draws conclusions to this dissertation by 
summarizing the contribution of this research and providing recommendations for future research. 


\section{CHAPTER 2}

\section{LITERATURE REVIEW}

This chapter provides a comprehensive literature review on the calibration of the HSM predictive models and prioritization of data variables. It first presents the different approaches and the sampling techniques used to estimate calibration factors. An extensive review of the current practices for determining the influence of explanatory variables is provided next.

\subsection{Calibration Factor}

This section presents a review of the studies that focused on calibration of the HSM's predictive models. The review is divided into the following four subsections based on the purpose of the studies: studies reflecting the importance of calibration factor, studies validating the importance of random site selection, studies estimating calibration factor for different jurisdictions, and studies evaluating the sample size criteria recommended in the HSM.

\subsubsection{Need for Calibration Factor}

The importance of calibration factor in crash prediction models was evaluated by Sun et al. (2006), Findley et al. (2012), and Young and Park (2013). Each study used different approaches to justify the importance of calibration factor.

Sun et al. (2006) calibrated the safety prediction models presented in the draft version of the HSM for rural two-lane roadways in Louisiana. To demonstrate the importance of calibration factor, the authors measured the percentage difference between observed and predicted crashes for three scenarios. Scenario 1 included CMFs for lane 
width, and shoulder width and type; Scenario 2 included CMFs for lane width, shoulder width and type, and driveway density; and Scenario 3 included CMFs for lane width and shoulder width and type, and calibration factor. The scenario that produced the smallest percentage difference between observed and predicted crashes was regarded as the most suitable scenario for predicting crashes. The study found that Scenario 3 that included calibration factor yielded the lowest difference of $5.22 \%$ compared to the differences of $52.09 \%$ in Scenario 1 and $10.59 \%$ in Scenario 2. The results, therefore, justified the significance of calibrated model in predicting crashes.

Findley et al. (2012) conducted a paired sample t-test to determine whether or not there is any statistically significant difference between observed and predicted crashes. The test statistic was computed using data from a total of 51 rural two-lane curve sections in North Carolina. A statistically significant difference was found between observed and predicted crashes on curves-only and composite (curves and tangents) sections at 5\% significance level. This result indicated the need to use a calibration factor other than 1.00 .

In the study by Young and Park (2013), crash prediction performance was evaluated between calibrated and uncalibrated models for three-leg unsignalized intersections, four-leg unsignalized intersections, and signalized intersections (combining three-leg and four-leg together) in the city of Regina, Saskatchewan. Several quantitative goodness-of-fit tests such as mean squared prediction error (MSPE), mean squared error (MSE), mean prediction bias (MPB), and Freeman-Tukey R-Squared $\left(R_{F T}^{2}\right)$ were applied in comparing the performance of calibrated and uncalibrated crash predictions. All the test measures had better fitted values (i.e., lower values of MSPE, MSE, and MPB, and 
higher value of $R_{F T}^{2}$ ) for the calibrated models compared to those for the uncalibrated models at all severity levels (total, fatal and injury (FI), and property damage only (PDO)), indicating the merit of calibration. In addition, the cumulative residual (CURE) plot was used to demonstrate the distribution of cumulative residuals (cumulative difference between observed and predicted crashes) against major road AADT. The residuals for uncalibrated models had extreme deviations from the $95 \%$ confidence interval bounds almost throughout the entire range of AADT values for all severity levels. On the other hand, the residuals for calibrated models had only slight deviations from the $95 \%$ confidence interval bounds for a narrow range of AADT values. The graphical plots also demonstrated the superior performance of the calibrated models over the uncalibrated models.

The findings of these studies suggest that the calibration of the HSM predictive models is an imperative step for successful implementation of the HSM.

\subsubsection{Random Site Selection}

A few studies investigated the impact of site selection bias. Sun et al. (2006) and Findley et al. (2012) studied the impact of deliberately selecting high crash sites versus selecting random sites on crash predictions.

Sun et al. (2006) used two control groups - one consisting of 26 randomly selected sites and the other consisting of 16 high crash sites, and derived the percentage difference between observed and predicted crashes for each group for three scenarios. As mentioned earlier, Scenario 1 included CMFs for lane width, and shoulder width and type; Scenario 2 included CMFs for lane width, shoulder width and type, and driveway density; and Scenario 3 included CMFs for lane width and shoulder width and type, and 
calibration factor. Table 2-1 presents the results from this study. The negative values indicate under-prediction of crashes and the positive values indicate over-prediction of crashes. It is observed that the differences between predicted and observed crashes were extremely high for high-crash sites compared to those for randomly selected sites for all the three scenarios. Even the calibrated model (i.e., Scenario 3) could not make a good approximation of predicted crash frequency when high crash sites were considered. The results, therefore, provided the evidence that considering only high-crash sites is likely to induce bias in estimating crash predictions.

TABLE 2-1 Summary of Results Showing the Effect of Site Selection Types (Sun et al., 2006)

\begin{tabular}{|l|c|c|}
\hline \multirow{2}{*}{ Scenario } & \multicolumn{2}{|c|}{$\begin{array}{r}\text { Percentage Difference between Predicted } \\
\text { and Observed Crashes }\end{array}$} \\
\cline { 2 - 3 } & $\begin{array}{r}\text { Randomly Selected Sites } \\
\text { High-Crash Sites }\end{array}$ & -263.77 \\
\hline $\begin{array}{l}\text { 1 - included CMFs for lane width, and } \\
\text { shoulder width and type }\end{array}$ & -52.03 & -136.84 \\
\hline $\begin{array}{l}\text { 2 - included CMFs for lane width, shoulder } \\
\text { width and type, and driveway density }\end{array}$ & 10.59 & -141.31 \\
\hline $\begin{array}{l}3 \text { - included CMFs for lane width and } \\
\text { shoulder width and type, and calibration } \\
\text { factor }\end{array}$ & 5.22 & \\
\hline
\end{tabular}

Findley et al. (2012) experimented on the degree of variation in calibration factors between random and non-random sites for rural two-lane curve sections in North Carolina. A total of 51 locations were considered for estimating calibration factors, where 25 locations were randomly selected and another 26 sites were HCLs. The calibration factor estimated by considering all the locations was 2.82 , whereas it was 4.50 when only HCLs were considered and 1.33 when only randomly selected locations were considered. The calibration factors estimated using HCLs alone was found to be 2.1 to 3.4 times the calibration factors estimated from randomly selected sites. It is, therefore, evident from 
the study results that calibration factors estimated from non-randomly selected sites would not give reliable crash prediction estimates.

\subsubsection{Sampling in States' HSM Calibration}

Table 2-2 presents the sample sizes used in the studies that calibrated the crash prediction models in the HSM for local jurisdictions. Note that the studies listed in Table 2-2 follow the chronological order based on their publication date. A description of the sampling processes used in the studies is also provided here.

TABLE 2-2 Sample Sizes Used in Calibrating the HSM Prediction Models

\begin{tabular}{|c|c|c|c|c|}
\hline State & Facility & \begin{tabular}{|c|}
$\begin{array}{c}\text { Number } \\
\text { of } \\
\text { Sites }\end{array}$ \\
\end{tabular} & $\begin{array}{c}\text { Total } \\
\text { Length } \\
\text { (mi) }\end{array}$ & $\begin{array}{c}\text { Number } \\
\text { of Crashes } \\
\text { per Year }\end{array}$ \\
\hline $\begin{array}{l}\text { Arezzo, Italy } \\
\text { (Martinelli } \\
\text { et al., 2009) }\end{array}$ & Rural two-lane, two-way roads & 8,379 & 583.0 & 134 \\
\hline \multirow{13}{*}{$\begin{array}{l}\text { Florida } \\
\text { (Srinivasan } \\
\text { et al., 2010) }\end{array}$} & Rural two-lane, two-way roads & 4,811 & 2121.0 & 977 \\
\hline & Rural multilane divided highways & 1,351 & 546.2 & 577 \\
\hline & Urban and suburban two-lane undivided arterials & 5,076 & 628.4 & 924 \\
\hline & Urban and suburban three-lane arterials with a TWLTL & 709 & 66.3 & 122 \\
\hline & Urban and suburban four-lane undivided arterials & 1,251 & 96.1 & 330 \\
\hline & Urban and suburban four-lane divided arterials & 7,506 & 970.6 & 2,885 \\
\hline & Urban and suburban five-lane arterials with a TWLTL & 2,868 & 253.6 & 1,005 \\
\hline & Rural two-lane three-leg stop-controlled intersections & 39 & $\mathrm{~N} / \mathrm{A}$ & 27 \\
\hline & Rural two-lane four-leg stop-controlled intersections & 24 & N/A & 22 \\
\hline & Rural two-lane four-leg signalized intersections & 28 & $\mathrm{~N} / \mathrm{A}$ & 44 \\
\hline & Rural multilane four-leg signalized intersections & 25 & N/A & 48 \\
\hline & Urban and suburban three-leg signalized intersections & 45 & N/A & 107 \\
\hline & Urban and suburban four-leg signalized intersections & 121 & $\mathrm{~N} / \mathrm{A}$ & 737 \\
\hline \multirow{9}{*}{$\begin{array}{l}\text { North } \\
\text { Carolina } \\
\text { (Srinivasan } \\
\text { and Carter, } \\
2011 \text { ) }\end{array}$} & Rural multilane divided highways & $\mathrm{N} / \mathrm{P}$ & 49.8 & 142 \\
\hline & Urban and suburban two-lane undivided arterials & $\mathrm{N} / \mathrm{P}$ & 59.4 & 269 \\
\hline & Urban and suburban three-lane arterials with a TWLTL & $\mathrm{N} / \mathrm{P}$ & 7.6 & 89 \\
\hline & Urban and suburban four-lane undivided arterials & $\mathrm{N} / \mathrm{P}$ & 15.3 & 478 \\
\hline & Urban and suburban four-lane divided arterials & $\mathrm{N} / \mathrm{P}$ & 15.5 & 281 \\
\hline & Urban and suburban five-lane arterials with a TWLTL & $\mathrm{N} / \mathrm{P}$ & 12.5 & 214 \\
\hline & Rural two-lane three-leg stop-controlled intersections & 133 & $\mathrm{~N} / \mathrm{A}$ & 63 \\
\hline & Rural two-lane four-leg stop-controlled intersections & 59 & $\mathrm{~N} / \mathrm{A}$ & 57 \\
\hline & Rural two-lane four-leg signalized intersections & 19 & $\mathrm{~N} / \mathrm{A}$ & 101 \\
\hline
\end{tabular}




\begin{tabular}{|c|c|c|c|c|}
\hline State & Facility & $\begin{array}{c}\text { Number } \\
\text { of } \\
\text { Sites }\end{array}$ & \begin{tabular}{|c|} 
Total \\
Length \\
(mi)
\end{tabular} & $\begin{array}{c}\text { Number } \\
\text { of Crashes } \\
\text { per Year }\end{array}$ \\
\hline & Rural multilane four-leg signalized intersections & 23 & N/A & 152 \\
\hline & Urban and suburban three-leg stop-controlled intersections & 73 & N/A & 85 \\
\hline & Urban and suburban four-leg stop-controlled intersections & 20 & N/A & 34 \\
\hline & Urban and suburban three-leg signalized intersections & 31 & N/A & 132 \\
\hline & Urban and suburban four-leg signalized intersections & 122 & $\mathrm{~N} / \mathrm{A}$ & 977 \\
\hline \multirow{2}{*}{$\begin{array}{l}\text { Louisiana } \\
\text { (Sun et al., } \\
\text { 2011) }\end{array}$} & Rural multilane undivided highways & $145-197$ & $60-71$ & 153 \\
\hline & Rural multilane divided highways & $342-441$ & $454-604$ & 1,559 \\
\hline \multirow{18}{*}{$\begin{array}{l}\text { Oregon } \\
\text { (Dixon et al., } \\
2012 \text { ) }\end{array}$} & Rural two-lane, two-way roads & 75 & $\mathrm{~N} / \mathrm{P}$ & 131 \\
\hline & Rural multilane undivided highways & 50 & $\mathrm{~N} / \mathrm{P}$ & 121 \\
\hline & Rural multilane divided highways & 19 & $\mathrm{~N} / \mathrm{P}$ & 19 \\
\hline & Urban and suburban two-lane undivided arterials & 491 & $\mathrm{~N} / \mathrm{P}$ & 126 \\
\hline & Urban and suburban three-lane arterials with a TWLTL & 205 & $\mathrm{~N} / \mathrm{P}$ & 72 \\
\hline & Urban and suburban four-lane undivided arterials & 375 & $\mathrm{~N} / \mathrm{P}$ & 169 \\
\hline & Urban and suburban four-lane divided arterials & 86 & $\mathrm{~N} / \mathrm{P}$ & 54 \\
\hline & Urban and suburban five-lane arterials with a TWLTL & 323 & $\mathrm{~N} / \mathrm{P}$ & 257 \\
\hline & Rural two-lane three-leg stop-controlled intersections & 200 & $\mathrm{~N} / \mathrm{A}$ & 36 \\
\hline & Rural two-lane four-leg stop-controlled intersections & 200 & N/A & 68 \\
\hline & Rural two-lane four-leg signalized intersections & 25 & N/A & 47 \\
\hline & Rural multilane three-leg stop-controlled intersections & 100 & N/A & 12 \\
\hline & Rural multilane four-leg stop-controlled intersections & 107 & N/A & 59 \\
\hline & Rural multilane four-leg signalized intersections & 34 & N/A & 52 \\
\hline & Urban and suburban three-leg stop-controlled intersections & 73 & N/A & 34 \\
\hline & Urban and suburban four-leg stop-controlled intersections & 48 & N/A & 35 \\
\hline & Urban and suburban three-leg signalized intersections & 49 & N/A & 107 \\
\hline & Urban and suburban four-leg signalized intersections & 57 & N/A & 230 \\
\hline $\begin{array}{l}\text { Illinois } \\
\text { (Williamson } \\
\text { and Zhou, } \\
\text { 2012) }\end{array}$ & Rural two-lane, two-way roads & 30 & $\mathrm{~N} / \mathrm{P}$ & 31 \\
\hline $\begin{array}{l}\text { Kansas } \\
\text { (Lubliner } \\
\text { and Schrock, } \\
\text { 2012) }\end{array}$ & Rural two-lane, two-way roads & 239 & 190.0 & 146 \\
\hline $\begin{array}{l}\text { Utah } \\
\text { (Brimley } \\
\text { et al., 2012) }\end{array}$ & Rural two-lane, two-way roads & 157 & $\mathrm{~N} / \mathrm{P}$ & 142 \\
\hline \multirow{2}{*}{$\begin{array}{l}\text { Toronto, } \\
\text { Canada } \\
\text { (Persaud et } \\
\text { al., 2012) }\end{array}$} & Urban and suburban three-leg signalized intersections & 343 & $\mathrm{~N} / \mathrm{A}$ & $>1,300$ \\
\hline & Urban and suburban four-leg signalized intersections & 1,691 & $\mathrm{~N} / \mathrm{A}$ & $>19,000$ \\
\hline \multirow{2}{*}{$\begin{array}{l}\text { Alabama } \\
\text { (Mehta and } \\
\text { Lou, 2013) }\end{array}$} & Rural two-lane, two-way roads & 5,991 & $\mathrm{~N} / \mathrm{P}$ & $\mathrm{N} / \mathrm{P}$ \\
\hline & Rural multilane divided highways & 4,000 & $\mathrm{~N} / \mathrm{P}$ & $\mathrm{N} / \mathrm{P}$ \\
\hline
\end{tabular}




\begin{tabular}{|c|c|c|c|c|}
\hline State & Facility & $\begin{array}{c}\begin{array}{c}\text { Number } \\
\text { of } \\
\text { Sites }\end{array} \\
\end{array}$ & \begin{tabular}{|c|} 
Total \\
Length \\
(mi) \\
\end{tabular} & $\begin{array}{c}\text { Number } \\
\text { of Crashes } \\
\text { per Year }\end{array}$ \\
\hline \multirow{13}{*}{$\begin{array}{l}\text { Missouri } \\
\text { (Sun et al., } \\
\text { 2013) }\end{array}$} & Rural two-lane, two-way roads & 196 & 107.8 & 101 \\
\hline & Rural multilane divided highways & 37 & 96.2 & 238 \\
\hline & Urban and suburban two-lane undivided arterials & 73 & 59.1 & 86 \\
\hline & Urban and suburban four-lane divided arterials & 66 & 70.0 & 189 \\
\hline & Urban and suburban five-lane arterials with a TWLTL & 59 & 37.8 & 251 \\
\hline & Rural two-lane three-leg stop-controlled intersections & 70 & N/A & 8 \\
\hline & Rural two-lane four-leg stop-controlled intersections & 70 & $\mathrm{~N} / \mathrm{A}$ & 16 \\
\hline & Rural multilane three-leg stop-controlled intersections & 70 & $\mathrm{~N} / \mathrm{A}$ & 15 \\
\hline & Rural multilane four-leg stop-controlled intersections & 70 & N/A & 31 \\
\hline & Urban and suburban three-leg stop-controlled intersections & 70 & N/A & 17 \\
\hline & Urban and suburban four-leg stop-controlled intersections & 70 & N/A & 60 \\
\hline & Urban and suburban three-leg signalized intersections & 35 & $\mathrm{~N} / \mathrm{A}$ & 177 \\
\hline & Urban and suburban four-leg signalized intersections & 35 & N/A & 449 \\
\hline \multirow{2}{*}{$\begin{array}{l}\text { Virginia } \\
\text { (Kweon et } \\
\text { al., 2014) }\end{array}$} & Rural multilane divided highways & 1,401 & 858.0 & 3,124 \\
\hline & Rural multilane four-leg signalized intersections & 127 & N/A & 706 \\
\hline $\begin{array}{l}\text { South } \\
\text { Dakota } \\
\text { (Qin et al., } \\
2014 \text { ) }\end{array}$ & Rural two-lane, two-way roads & 657 & 787.0 & 30 \\
\hline \multirow{18}{*}{$\begin{array}{l}\text { Maryland } \\
\text { (Shin et al., } \\
\text { 2014) }\end{array}$} & Rural two-lane, two-way roads & 251 & $\mathrm{~N} / \mathrm{P}$ & 153 \\
\hline & Rural multilane undivided highways & 19 & $\mathrm{~N} / \mathrm{P}$ & 14 \\
\hline & Rural multilane divided highways & 160 & $\mathrm{~N} / \mathrm{P}$ & 105 \\
\hline & Urban and suburban two-lane undivided arterials & 252 & $\mathrm{~N} / \mathrm{P}$ & 120 \\
\hline & Urban and suburban three-lane arterials with a TWLTL & 138 & $\mathrm{~N} / \mathrm{P}$ & 110 \\
\hline & Urban and suburban four-lane undivided arterials & 145 & $\mathrm{~N} / \mathrm{P}$ & 197 \\
\hline & Urban and suburban four-lane divided arterials & 244 & $\mathrm{~N} / \mathrm{P}$ & 218 \\
\hline & Urban and suburban five-lane arterials with a TWLTL & 115 & $\mathrm{~N} / \mathrm{P}$ & 419 \\
\hline & Rural two-lane three-leg stop-controlled intersections & 162 & $\mathrm{~N} / \mathrm{A}$ & 34 \\
\hline & Rural two-lane four-leg stop-controlled intersections & 115 & $\mathrm{~N} / \mathrm{A}$ & 47 \\
\hline & Rural two-lane four-leg signalized intersections & 67 & $\mathrm{~N} / \mathrm{A}$ & 87 \\
\hline & Rural multilane three-leg stop-controlled intersections & 26 & $\mathrm{~N} / \mathrm{A}$ & 12 \\
\hline & Rural multilane four-leg stop-controlled intersections & 10 & N/A & 10 \\
\hline & Rural multilane four-leg signalized intersections & 35 & $\mathrm{~N} / \mathrm{A}$ & 77 \\
\hline & Urban and suburban three-leg stop-controlled intersections & 152 & N/A & 34 \\
\hline & Urban and suburban four-leg stop-controlled intersections & 90 & $\mathrm{~N} / \mathrm{A}$ & 58 \\
\hline & Urban and suburban three-leg signalized intersections & 167 & $\mathrm{~N} / \mathrm{A}$ & 263 \\
\hline & Urban and suburban four-leg signalized intersections & 244 & N/A & 588 \\
\hline
\end{tabular}

N/A - Information on roadway length is not applicable for intersections.

$\mathrm{N} / \mathrm{P}$ - Information on roadway length was not provided. 
Arezzo, Italy

Martinelli et al. (2009) evaluated the transferability of the crash prediction model for rural two-lane roads in the Arezzo province of Italy. The calibration process was carried out using three years (2002-2004) of crash data collected on about $938 \mathrm{~km}$ of road network. In particular, a total of 8,379 homogeneous sections were created with an average length of $367 \mathrm{ft}(0.07 \mathrm{mi})$. The observed crash frequency was 134 crashes per year.

Florida

Srinivasan et al. (2010) calibrated the SPFs in the HSM to Florida data for FI crashes. Calibration factors were derived for seven roadway segment types using four years (2005-2008) of crash data and for six intersection types using five years (20052009) of crash data. The study derived calibration factors by collecting data on state roads from the entire state rather than just conforming to the minimum sample size criteria. However, three-leg and four-leg stop-controlled intersections on rural two-lane roads and four-leg stop-controlled intersections on rural multilane highways did not meet the minimum sample size requirement of 30 to 50 sites with at least 100 crashes per year. The study also investigated whether segmentation by geographic area or by demographic characteristics would result in a large variation in calibration factors within the state of Florida. The segmentation of road network either by seven Florida Department of Transportation (FDOT) districts or by four groups of population density resulted in insufficient data to derive calibration factors for some facilities such as urban three-lane arterials with a TWLTL, urban four-lane divided roads, and urban five-lane arterials with a TWLTL. Although the study used a huge data set to estimate calibration factors, it did 
not investigate whether there was any difference in calibration factors developed using the entire data set and that using the minimum sample size.

\section{North Carolina}

Srinivasan and Carter (2011) calibrated the HSM predictive models for North Carolina. The study used three years (2007-2009) of traffic and crash data to develop calibration factors for six roadway segment types and eight intersection types. It was found that not all facilities had enough data to comply with the HSM calibration sample size criteria. For example, three-leg and four-leg stop-controlled intersections on rural two-lane highways and urban and suburban arterials did not satisfy the minimum threshold of 100 crashes per year. On the other hand, fewer than 30 four-leg signalized intersections on both rural two-lane and rural multilane facilities experienced a minimum

of 100 crashes per year. This indicates that the criteria recommended for calibration could be relaxed based on local characteristics.

\section{Louisiana}

Sun et al. (2011) derived calibration factors for rural four-lane undivided and divided facilities using five years (2003-2007) of data from the state of Louisiana. The study used the entire data maintained by the Louisiana Department of Transportation and Development (LaDOTD). Although the number of sites varied by years, the sample size used in the study exceeded the HSM's minimum recommended criteria.

\section{Oregon}

Dixon et al. (2012) developed calibration factors to apply the HSM predictive method in the state of Oregon. Calibration factors were determined for all the segment and intersection types discussed in the HSM, using three years (2004-2006) of crash data. 
The study adopted some rules of thumb to extract a sufficient number of sites from the entire state to ensure the minimum crash frequency of 100 crashes per year. For example, as many as 200 sites were selected for both three-leg and four-leg stop-controlled intersections on rural two-lane highways and a minimum of 100 sites were selected for both three-leg and four-leg stop-controlled intersections on rural multilane highways. The sample size for rural two-lane undivided roads comprised of 75 segments each approximately two-mile long and that for rural multilane divided roads comprised of 50 segments varying from 0.50 to approximately 2 miles. In most cases, samples with more than 200 sites were taken for urban and suburban arterials. Although sufficiently large samples were drawn for most of the facility types, the crash frequency threshold in several facilities were far below than 100 annual crashes. This indicates that sample size with a threshold of 100 crashes per year might not always be required; rather, a decent number of sites might work well to produce a good-quality calibration factor within the budget constraint.

Illinois

Williamson and Zhou (2012) calibrated two SPFs, the HSM-default SPF and the Illinois-specific SPF developed by Tegge et al. (2010), for rural two-lane, two-way roads in Illinois. The study used merely a sample of 30 randomly selected sites for the calibration process. These 30 sites experienced a very low annual crash frequency of 31 crashes, which is lower than the minimum threshold of 100 crashes per year.

\section{Kansas}

Lubliner and Schrock (2012) used a different approach to follow the sample size criteria for deriving calibration factor for rural two-lane, two-way roads in Kansas. The 
authors adopted a two-step approach for selecting sample size. First, a total of 41 ten-mile sections were randomly selected. Based on the distribution of annual crash frequency among these sections, 19 ten-mile sections were found to be required to meet the minimum threshold of 100 crashes per year at 90\% confidence level. Therefore, 19 tenmile sections were then randomly selected, yielding 239 homogenous segments with 146 crashes per year.

\section{Utah}

Brimley et al. (2012) calibrated the HSM-default SPFs for rural two-lane, twoway roads in Utah. The calibration data set consisted of 157 roadway segments that experienced 142 crashes per year based on three years (2005-2007) of crash data. Note that although the study fulfilled the sample size criteria, AADT values less than 10,000 veh/day and speed limits greater than $45 \mathrm{mph}$ were the pruning criteria used to select the segments for estimating the calibration factor.

\section{Toronto, Canada}

Persaud et al. (2012) calibrated the HSM-default SPFs and the locally developed SPFs for multi-vehicle and rear-end crashes at three-leg and four-leg signalized intersections in Toronto. The data set in the study consisted of 343 three-leg signalized intersections and 1,691 four-leg signalized intersections, where the observed crash frequencies were significantly higher than the minimum threshold of 100 crashes per year.

Alabama

Mehta and Lou (2013) collected a significant amount of data for calibrating the HSM-default SPFs to rural roadway facilities in the state of Alabama. Calibration factors 
were developed for rural two-lane, two-way roads using a data set consisting of 5,991 sites with segment lengths varying from 0.05 to 0.68 miles, and for four-lane divided highways using a data set consisting of 4,000 sites with segment lengths varying from 0.01 to 6.59 miles. Furthermore, the reliability of the calibration factors was tested by using a different sample that comprised of 3,000 sites for rural two-lane, two-way roads and 2,000 sites for four-lane divided highways. The study, however, did not verify the reliability of calibration factors with the minimum sample size criteria.

Missouri

Sun et al. (2013) developed calibration factors for five segment and eight intersection types for the state of Missouri. The study adopted different sampling strategies for rural and urban roadway facilities. For rural two-way, two-lane roads and rural multilane divided highways, the sampling was done by randomly extracting five samples with a minimum segment length of 0.50 miles from each of the seven Missouri Department of Transportation (MoDOT) districts. The resulting 35 samples for each facility were then divided into homogeneous segments. However, for urban and suburban site types, segments with a minimum length of 0.25 miles were selected. In the case of three-leg and four-leg stop-controlled intersections on both rural and urban facilities, the sampling process extracted 10 intersections from each of the seven MoDOT districts resulting in a total of 70 intersections. However, the observed crash frequency at all the stop-controlled intersection types was considerably less than 100 crashes per year. Similar to the several earlier discussed studies, the study also could not meet the minimum sample size criteria for some facilities. 


\section{Virginia}

Kweon et al. (2014) presented specific guidelines to derive calibration factors for the application of the HSM predictive method in Virginia. Two facilities, rural four-lane divided segments and four-leg signalized intersections in rural multilane highways, were studied to develop calibration factors for each district of the Virginia Department of Transportation (VDOT). The database maintained by the VDOT did not have adequate samples for intersections to meet the sample size criteria for a majority of the districts. Therefore, an alternative approach was used to estimate district-wide calibration factors. The approach involved calculating two calibration factors: (1) district-specific calibration factor, and (2) statewide calibration factor. The district-specific calibration factor was estimated for each district using five years of data and the statewide calibration factor was estimated for each year of the analysis period. The product of these two calibration factors was termed as district-wide calibration factor and considered to conform to the minimum sample size requirements.

\section{South Dakota}

Qin et al. (2014) calibrated crash prediction models for rural two-lane, two-way roads in South Dakota. The study used 657 homogeneous segments comprising 750 miles of roadways for calculating calibration factors. These 657 segments were found to experience only 30 crashes per year.

\section{Maryland}

Shin et al. (2014) developed calibration factors for all the segment and intersection types in the state of Maryland. All the segment and intersection types met the minimum sample size criteria except for rural four-lane undivided roads. Although the 
sample size for three-leg and four-leg stop-controlled intersections on rural two-lane and multilane highways was sufficiently large in most cases, the annual crash frequency was far below the recommended minimum criterion of 100 crashes per year. Note that the entire data set available in the state database was not used in the study; rather, samples were selected based on $90 \%$ statistical confidence level and $5 \%$ margin of errors from the population data set.

\subsubsection{Evaluation of Calibration Sample Size Criteria}

Since the release of the first edition of the HSM in 2010, several studies have focused on evaluating the HSM recommended sample size criteria. Banihashemi (2011) first verified the criterion of minimum crash frequency by randomly selecting sites that experienced 100,150,200, 250, and 300 crashes per year. The estimated probability that the calibration factor using the samples would be within a $10 \%$ difference from the overall calibration factor (calculated from the entire data set) was $47 \%, 60 \%, 63 \%, 69 \%$, and $75 \%$, respectively. The findings of the study, therefore, revealed that the minimum frequency of 100 crashes per year might not be adequate to estimate a reliable calibration factor.

More recently, Banihashemi (2012) and Alluri et al. (2014) conducted a sensitivity analysis to evaluate the recommended sample size criteria for calibrating the models using data from Washington State and Florida, respectively. While Banihashemi (2012) was restricted to only segments, Alluri et al. (2014) conducted the analysis for both segments and intersections. Both the studies compared the effects of different sample sizes on the reliability of the estimated calibration factor. Banihashemi (2012) selected random samples based on varying length percentages of total roadway miles. For 
example, samples for rural two-lane undivided roadways consisted of $5 \%, 10 \%, 20 \%$, $30 \%, 40 \%$, and $50 \%$ of the total 4,812 miles of rural two-lane undivided roadways. Alluri et al. (2014) selected random samples by number of sites. For example, samples for rural two-lane undivided roadways were comprised of 50,75, 100, 125, 150, 175, 200, 225, 250, 275, and 300 segments. The calibration factors developed with different sample sizes were compared with the calibration factor calculated using the complete data set and the variation was estimated in terms of probability. Based on the results of probability estimates, both the studies recommended different sample sizes for different site types.

Trieu et al. (2014) also performed a sensitivity analysis to justify the HSM criterion of selecting 30 to 50 sites for calibration process. The analysis was carried out based on six sets of sample data constituting $10 \%, 20 \%, 30 \%, 40 \%, 50 \%$, and $60 \%$ of total number of sites. For each percentage, 500 samples were generated by randomly resampling the data through Monte Carlo simulation. The sensitivity was measured in terms of percent error using the following equation:

$$
\text { Percent Error }=\frac{C_{r}-C_{r_{-} \text {target }}}{C_{r_{-} \text {target }}} \times 100 \%
$$

where $C_{r_{-} \text {target }}$ is the target calibration factor computed using the entire data set and $C_{r}$ is the calibration factor computed using data for each sample.

The distribution of percent error among 500 calibration factors for each percentage group showed that the percent error decreased with increasing number of sites. For example, a sample size consisting of $10 \%$ data from the entire data set produced $67 \%$ calibration factors within $10 \%$ error; whereas, almost all (i.e., $99.8 \%$ ) the calibration 
factors estimated using $50 \%$ of the entire data set were obtained within $10 \%$ error range from the target calibration factor. The study concluded that a sample size consisting of 30 to 50 sites might not be enough to produce reliable calibration factors and called for revision of this criterion.

Shin et al. (2014) employed the following four sampling strategies for extracting samples from the population:

- Scenario 1: Samples were drawn such that the HSM recommendations of a minimum of 30 segments with at least 100 crashes per year were met. Segments shorter than 0.1 miles on rural highways and segments shorter than 0.04 miles on urban and suburban arterials were not included.

- Scenario 2: Sample size was determined based on the following equations:

$$
n=\frac{n_{0} \times N}{n_{0}+N-1}
$$

with

$$
n_{0}=\frac{Z^{2} \times P \times(1-P)}{e^{2}}
$$

where,

$$
\begin{aligned}
n= & \text { minimum sample size } \\
N= & \text { population, } \\
Z= & \text { area under normal curve corresponding to the desired confidence } \\
& \text { level, } \\
P= & \text { true proportion of factor in the population, and } \\
e= & \text { margin of error. }
\end{aligned}
$$


A $90 \%$ confidence level, $50 \%$ true proportion of factor, and 5\% margin of error in the population were used to determine sample size. Samples were selected with the minimum segment length threshold given in Scenario 1.

- Scenario 3: Samples were drawn based on disproportionate stratified random sampling technique where data were stratified by segment length and crash frequency.

- Scenario 4: Sample size was determined by combining Scenarios 1 through 3.

It should be obvious that the sample size extracted for Scenario 4 was the largest as it combined all the unique segments from Scenarios 1, 2, and 3. Also, in most of the cases, the sample size in Scenario 2 was larger than the sample sizes in Scenarios 1 and 3. Note that the intersection data were collected for Scenarios 1 and 2. The study evaluated the four scenarios using the following methods:

- range of calibration factors;

- absolute value of difference of calibration factors from 1.00;

- coefficient of variation of normalized crashes; and

- ratio of coefficient of variation of normalized crashes in samples to coefficient of variation of normalized crashes in the entire population.

None of these methods could draw a straight-cut conclusion on the best scenario for estimating reliable calibration factors. The authors preferred Scenario 2 to other scenarios because samples in Scenario 2 were extracted based on minimum sample size, crash frequency, and segment length thresholds, and statistical confidence level. 


\subsection{Data Prioritization}

A review of the techniques used to determine variable importance is provided in this section. The techniques are broadly divided into two groups: traditional approaches and data mining approaches.

\subsubsection{Traditional Approaches}

Traditionally, the focus of crash analysis was to establish relationship between crash frequency and roadway geometry, traffic, and environmental factors for various roadway entities. Usually, a single "parsimonious" model that incorporates only a few statistically significant (say, at a level of significance of 0.05 ) variables is developed. The negative binomial (NB) regression technique is typically favored by researchers to develop crash frequency models (e.g., Shankar et al., 1995; Hadi et al., 1995; Poch and Mannering, 1996; Milton and Mannering, 1996; Abdel-Aty and Radwan, 2000; Sawalha and Sayed, 2001; Hauer et al., 2004; Caliendo et al., 2007; Montella et al., 2008; Easa and You, 2009; Cafiso et al., 2010; Tegge et al., 2010). However, limited attempts were made in the studies to determine the impact of each variable on the performance of crash prediction models. Montella et al. (2008) developed several single-variable models (including the intercept) using the NB method to investigate the possible influence of each explanatory variable on crash predictions. The effect of the individual predictor variables were determined by the goodness-of-fit measure of the models.

Recently, several studies conducted sensitivity analysis to determine the impact of variables on crash predictions. For example, Findley et al. (2012) performed sensitivity analysis to identify the variables that have the most significant effect on crashes on rural two-lane horizontal curves in North Carolina. Crashes were separately predicted by 
changing the values of one variable at a time at its maximum, minimum, mean, and actual field-measured values. The difference in predicted crashes using the maximum and minimum value of a variable was compared with the difference in predicted crashes using the mean and actual field-measured values of that variable. A comparative evaluation of these two differences found that AADT, curve length, and curve radius were the most important factors in predicting crash frequency on horizontal curve sections.

Alluri and Ogle (2012) also conducted sensitivity analysis to assess the influence of variations of one variable on the predicted crash frequency as a function of AADT on rural two-way two-lane roads in Georgia. The authors conducted three types of sensitivity analysis: (a) effect of variation of AADT on the predicted crash frequency; (b) effect of variations of each variable on predicted crash frequency if means of all other CMFs were considered; and (c) effect of variations of each variable on predicted crash frequency if all other variables were assumed to be base conditions.

Furthermore, Jalayer and Zhou (2013) examined the effect of data variables included in the HSM predictive method using both mathematical and graphical sensitivity analysis tools. Mathematically, the sensitivity of a variable was determined based on the following equation:

$$
\text { Sensitivity }_{i}=\frac{\text { Output }_{\text {max }, i}-\text { Output }_{\text {min }, i}}{\text { Output }_{\text {nominal }, i}}
$$

where,

$$
\begin{aligned}
& \text { Sensitivity }_{i}=\text { sensitivity of the variable } i, \\
& \text { Output }_{\text {max }, i}=\text { predicted crashes using maximum value of the variable } i, \\
& \text { Output }_{\text {min, } i} \quad=\text { predicted crashes using minimum value of the variable } i, \text { and }
\end{aligned}
$$


Output $_{\text {nominal, } i}=$ predicted crashes using nominal or base-case value of the variable $i$.

The graphical method measured the gradient of the line plot of predicted crashes against the gradual change in values of a variable. Both the methods provided similar ranking of the variables. The factors including AADT, major industrial driveway, and major commercial driveway were found to have significant effects on crash prediction for urban and suburban two-lane undivided arterials. On the other hand, the presence of onstreet parking, offset to roadside fixed objects, proportion of curb length with on-street parking were found to have the least effect on crash predictions.

Apart from the above methods, Akgüngör and Yıldız (2007) employed fractional factorial technique to evaluate sensitivity of the predictor variables included in the crash prediction model developed by Zegeer et al. (1987). The procedure determined both individual and two-way interaction effects of the predictor variables. This approach differs from the earlier discussed one-factor-at-a-time sensitivity analysis approach as it considers different combinations of maximum and minimum values of predictor variables at the same time. Based on the absolute value of the effects at the three- and twostandard deviation thresholds, variables were categorized into groups of primary importance (such as average daily traffic (ADT)) and of secondary importance (such as lane width, paved shoulder width, median roadside hazard rating, and their interactions with ADT), respectively. However, with increasingly more than two levels of variables, it becomes difficult to analyze the effects of variables using the fractional factorial method. 


\subsubsection{Data Mining Approaches}

Data mining is defined as "the science of extracting useful information from large data sets or databases" (Hand et al., 2001). Decision tree, commonly known as classification and regression tree (CART) (Breiman et al., 1984), is one of the most widely used data mining approaches (Williams, 2011). A decision tree develops prediction models based on 'binary recursive partitioning' of the data set. The process can be demonstrated using an inverted tree structure, with root at the top and leaves at the bottom, as shown in Figure 2-1. The root node represents the entire data set. The root node is then divided into two child nodes based on the value of an independent variable (splitter) that brings maximum homogeneity in the child nodes. Each of the child nodes then acts as parent node and the process is repeated until a pre-specified criterion is reached. Each successive node in the decision tree consists of smaller and smaller subsets of observations. The nodes where trees cease to grow are called terminal nodes, or leaves. The mean values provided by observations in the terminal nodes are the predictions (Chrysostomou et al., 2011; Freidl and Brodley, 1997; Kashani and Mohaymany, 2011; Williams, 2011). 


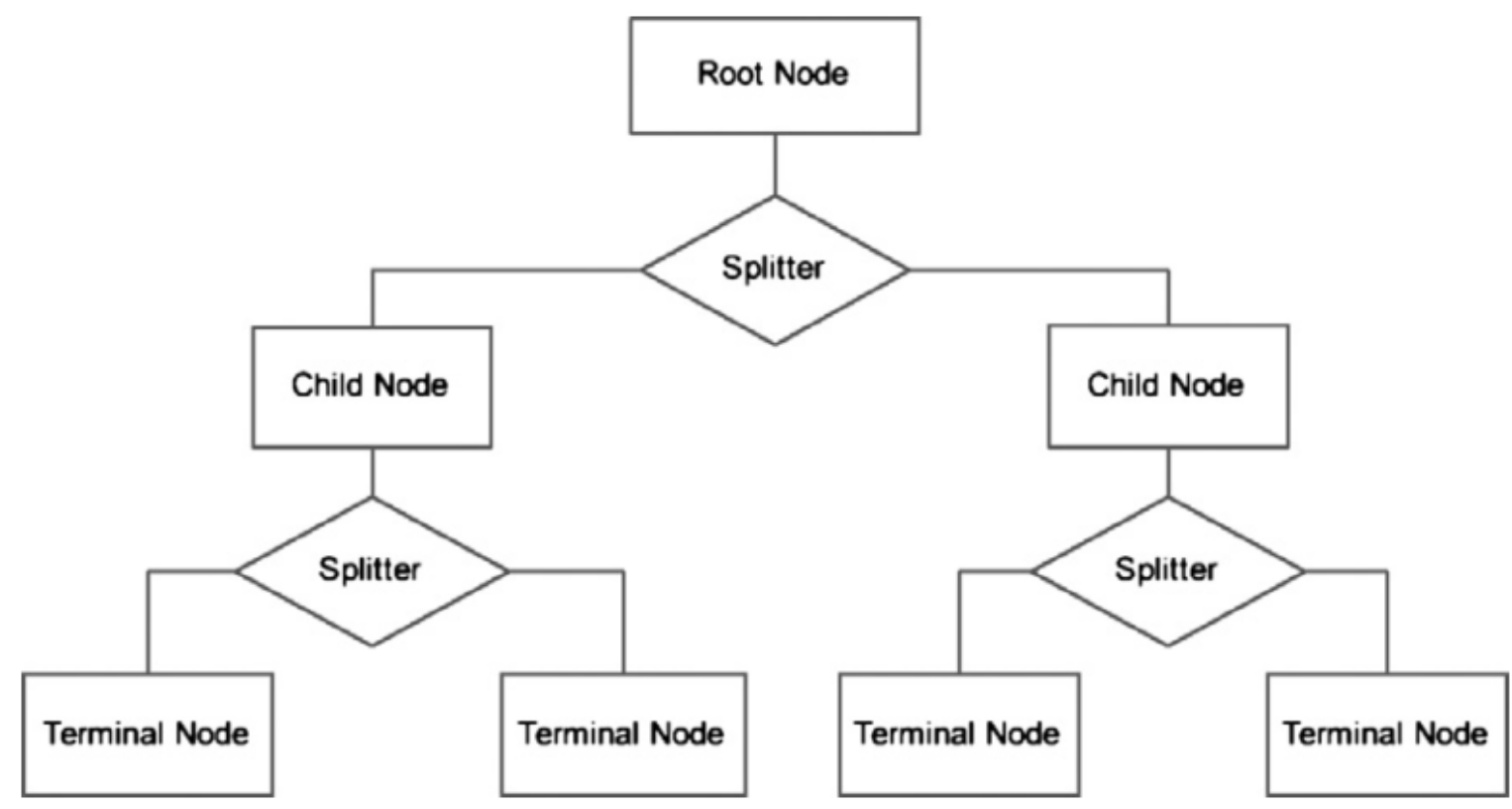

FIGURE 2-1 Illustration of a Decision Tree (Kashani and Mohaymany, 2011)

The decision tree method provides several advantages over regression analysis as follows (De'ath, 2007; Elith et al., 2008):

- Complex, non-linear relationship can be fitted very easily.

- The functional form between the predictor variables and the response variable does not need to be pre-specified.

- Missing data and outliers can be handled very well.

- No prior data transformation (e.g., logarithmic transformation of variables) is required.

- Influence of each predictor variable can readily be measured.

- Interactions between predictor variables can be automatically modeled.

A number of studies exploited the advantages of CART models in explaining the relation between crash characteristics and predictor variables. For example, Karlaftis and Golias (2002) developed a CART model, also referred to as hierarchical tree-based 
regression (HTBR) model, to estimate the relative contribution of variables on crash frequency for rural two-lane and multilane roads in Indiana. The study concluded that the HTBR models provided several advantages over traditional statistical models, which include variable importance measures, improved computational power, and an easy interpretation of the output. Yan and Radwan (2006) analyzed crashes involving two vehicles at signalized intersections by developing two classification tree models; one was formed to obtain the causal features resulting in rear-end crashes compared to non-rearend crashes, and the other was built to identify the factors attributed to at-fault drivers/vehicles versus not-at-fault drivers/vehicles for rear-end crashes. Chang and Wang (2006), and Kashani and Mohaymany (2011) applied the CART method to quantify the effects of vehicle, driver, and crash attributes on injury severity. Elmitiny et al. (2010) fitted a CART model to analyze the behavioral patterns of driver's stop-and-go situations and red-light running violations at an intersection.

A single decision tree model, however, can sometimes be unstable and produce output with high variance (Das et al., 2009; Zhang et al., 2005). The "ensemble of trees" approach is a step forward to reduce prediction error and exhibit more stability than the single decision tree model (Das et al., 2009; De'ath, 2007; Elith et al., 2008). In ensemble, many models are fitted based on some learning algorithms and their predictions are combined. Thus, more robust estimates of prediction are possible by the ensemble technique compared to relatively poor prediction by a single model (Strobol et al., 2009).

Random forests $(\mathrm{RF})$ and boosted regression trees (BRT) are the two common ensemble approaches. In RF, predictions are computed by fitting a number of trees 
(typically $50-1,000$ ) using a bootstrap sample of data and a subset of predictors. The RF algorithm has been increasingly applied in fields outside of transportation such as in mapping (Puissant et al., 2014), medicine (Özçift, 2011), ecology (Vincenzi et al., 2011) etc., to rank influential variables. In transportation, Harb et al. (2009) used RF technique to estimate the relative importance of variables on the binary outcome of drivers' crash avoidance maneuvers. Das et al. (2009) used a modified version of the RF method to measure variable importance and rank the variables affecting crash severity on urban arterials. Abdel-Aty and Haleem (2011) applied random forests method to determine the importance of the explanatory variables in predicting angle crash frequency at unsignalized intersections.

Alluri et al. (2014) prioritized the variables in the HSM for several segment and intersection subtypes using random forests algorithm. Based on the node purity values, the variables were ranked and grouped into three categories: variables of primary importance, variables of secondary importance, and variables of lesser importance. For each site type, the AADT variable (i.e., AADT for segments and major-road and minorroad AADT for intersections) had the highest node purity value and, therefore, was categorized as variables of primary importance. All other variables with node purity values within $15 \%$ of the highest node purity value were identified as the variables of secondary importance. The remaining variables (i.e., variables with node purity values less than $15 \%$ of the highest node purity value) were identified as the variables of lesser importance.

BRT is a relatively new and improved data mining technique applied in various fields including ecology (De'ath, 2007; Elith et al., 2008; Esther et al., 2014; Hale et al., 
2014), epidemiology (Cheong et al., 2014, Ellis et al., 2013; Neuman et al., 2004), soil mapping (Jafari et al., 2014; Lemercier et al., 2012), agriculture (Etter et al., 2006; Gellrich et al., 2008; Müller et al., 2013), and fisheries (Froeschke, 2011). Unlike random forests, the BRT method produces the assembly of trees with a slow learning rate and in a sequential manner to extract more variability in the data. Besides the advantages of other decision tree based models, one major advantage of the BRT approach is that it can also rigorously deal with different types of response variable (for example, binomial, count, normal, etc.) with the use of appropriate and robust loss function (Elith et al., 2008).

Chung (2013) explored the potentiality of the BRT approach in modeling complex, non-linear, and highly dispersed crash data. Several BRT models were developed to investigate the impact of the contributing factors on injury severity (i.e., fatal vs. non-fatal crashes) of single-vehicle motorcycle crashes in Taiwan. The output of the models provided variable importance and demonstrated marginal effects of the variables as well as interactions between them. The study concluded that since crash analysis usually involves a lot of explanatory variables and crash events are distinctive, the slow and sequential learning process of the BRT method would be able to give more emphasis on hard-to-fit observations for crash analysis.

\subsection{Summary}

A comprehensive review of studies on calibration factor estimation was presented. The review mainly focused on sample size used for estimating calibration factors. The HSM recommends developing calibration factors using 30 to 50 randomly selected sites that experienced at least 100 crashes per year. Most of the studies encountered the problem of inadequate sample size for calibration, either having fewer 
than 30 sites or having sites that experienced fewer than 100 crashes per year. A few studies proposed alternative ways to address the issue with limited sample size.

In addition, different techniques to measure variable importance were reviewed. The goal of this review was to explore the capabilities and limitations of the current practices in determining the impact of variables on crash predictions. The review included the conventional NB regression method, sensitivity analysis, and fractional factorial method, categorized as traditional approaches, and decision tree based methods, categorized as data mining approaches. The NB regression and sensitivity measures determined the influence of predictor variables by changing the value of one predictor variable at a time, whereas the fractional factorial method determined the effect of a variable with possible combinations of all other variables. However, fractional factorial would be difficult to apply when variables have more than two levels of categories, or when variables are continuous. Unlike the traditional approaches, the tree-based models provide an output of variable importance. 


\section{CHAPTER 3}

\section{DATA COLLECTION AND PREPARATION}

This chapter describes the data collection and preparation efforts undertaken in this dissertation research. The data needs for applying the HSM predictive models for urban and suburban arterials are discussed first. The data requirements comprise roadway and intersection characteristics, traffic control features, traffic volume, and crash frequency statistics for roadway segments and intersections. The following sections present the data set preparation efforts for both segments and intersections.

\subsection{Data Needs}

Table 3-1 presents the list of HSM variables with their specified level of data needs for both roadway segments and intersections on urban and suburban arterials in the HSM. The HSM categorizes each variable as either required or desirable. Required variables are those that are considered to have high impact on crash occurrence (Srinivasan et al., 2013). The HSM recommends using actual data for the required variables. Desirable variables are those that are considered as being not as sensitive to crash propensity (Srinivasan et al., 2013). When data for desirable variables are unavailable, the HSM suggests using default values based on agency's policy. In this research, data for all the variables were collected, either from the existing databases or from aerial images.

\subsection{Roadway Segment Data}

The RCI database maintained by the FDOT Transportation Statistics Office is the primary source of information for the variables identified in Table 3-1. The RCI is a 
comprehensive database that maintains over one million records on various physical and administrative data variables for the road network in Florida.

TABLE 3-1 Data Variables Identified in the HSM for Urban and Suburban Arterials (AASHTO, 2010)

\begin{tabular}{|c|c|c|}
\hline Facility Type & Data Variable & Data Needs \\
\hline \multirow{15}{*}{ Roadway Segments } & AADT & Required \\
\hline & Number of through traffic lanes & Required \\
\hline & Presence of TWLTL & Required \\
\hline & Presence of median & Required \\
\hline & Median width & Required \\
\hline & Number of driveways by land-use type & Required \\
\hline & Land use & Required \\
\hline & Speed limit & Required \\
\hline & Presence of on-street parking & Required \\
\hline & Type of on-street parking & Required \\
\hline & Curb length with on-street parking & Required \\
\hline & Roadside fixed object density & Desirable \\
\hline & Offset to fixed object & Desirable \\
\hline & Presence of lighting & Desirable \\
\hline & Use of automated speed enforcement & Desirable \\
\hline \multirow{17}{*}{ Intersections } & Area Type & Required \\
\hline & Type of intersection & Required \\
\hline & Type of traffic control & Required \\
\hline & AADT for major road & Required \\
\hline & AADT for minor road & Required \\
\hline & Number of approaches with left-turn lanes & Required \\
\hline & Number of approaches with right-turn lanes & Required \\
\hline & Presence of lighting & Required \\
\hline & Presence of left-turn signal phasing & Required \\
\hline & Type of left-turn signal phasing & Required \\
\hline & Presence of RTOR signal operation & Required \\
\hline & Presence of red-light cameras & Required \\
\hline & Number of bus stops within $1,000 \mathrm{ft}$ of intersection & Desirable \\
\hline & Presence of school within $1,000 \mathrm{ft}$ of intersection & Desirable \\
\hline & $\begin{array}{l}\text { Number of alcohol sales establishments within } 1,000 \mathrm{ft} \\
\text { of intersection }\end{array}$ & Desirable \\
\hline & Pedestrian activity level/ Pedestrian crossing volume & Desirable \\
\hline & Maximum number of lanes crossed by pedestrians & Desirable \\
\hline
\end{tabular}


The RCI includes data for roadway segments that are part of Florida's state highway system (SHS), segments that are currently being constructed and yet to be added as part of the SHS, segments that are physically removed, segments that are no longer maintained by the FDOT, county or city roads, historic roads, local roads, and exclusive roads (ramps, frontage roads etc.). Segments that are currently not part of the SHS do not have complete traffic and geometry data in the RCI. In addition, county and city roads, historic roads, and local roads do not conform to the specific roadway types described in the HSM. Therefore, all the analyses in this research were conducted by extracting data for the segments that are part of the SHS in Florida. Roadway geometric characteristics data were retrieved from RCI for the most recent year (i.e., 2012) and traffic data were extracted for a total of eight years from 2005 to 2012 from the corresponding year's RCI database.

After selecting the segments on SHS, the following steps were undertaken: (a) classify segments into the HSM-designated site types; (b) generate homogeneous segments for each site type; and (c) collect and process information for the data variables identified in the HSM. Each of these steps is described in the following subsections.

\subsubsection{Classification of Segments}

The HSM presents predictive models for the following urban and suburban arterial segment types (AASHTO, 2010):

- Two-lane undivided arterials: "a roadway consisting of two lanes with a continuous cross-section providing two directions of travel in which the lanes are not physically separated by either distance or a barrier." 
- Three-lane arterials with a center TWLTL: "a roadway consisting of three lanes with a continuous cross-section providing two directions of travel in which the center lane is a TWLTL."

- Four-lane undivided arterials: "a roadway consisting of four lanes with a continuous cross-section providing two directions of travel in which the lanes are not physically separated by either distance or a barrier."

- Four-lane divided arterials: "a roadway consisting of four lanes with a continuous cross-section providing two directions of travel in which the lanes are physically separated by either distance or a barrier."

- Five-lane arterials including a center TWLTL: "a roadway consisting of five lanes with a continuous cross-section providing two directions of travel in which the center lane is a TWLTL."

To categorize roadway segments into the particular urban and suburban arterial sections, the following five features in the RCI were used. Note that the description of the variable is provided in parentheses (FDOT, 2012).

- URBSIZE (area type),

- FUNCLASS (functional classification),

- NOLANES (number of lanes),

- TYPEROAD (type of road), and

- RDMEDIAN (type of median).

The URBSIZE variable in the RCI determines whether a roadway segment is located in an urban or a rural area. The variable has the following distinct levels to designate an area by population size (the one-digit codes are the RCI codes): 
- 1 - Rural

- 2 - Small urban (5,000 - 49,999 population)

- 3 - Small urbanized (50,000 - 199,999 population)

- 4 - Large urbanized (200,000 - 499,999 population)

- 5 - Metropolitan (500,000 or more population)

As such, segments within an area of at least 5,000 inhabitants (i.e., segments represented by codes "2", "3", "4", and "5") were designated as urban and suburban roadway segments.

The FUNCLASS feature of the RCI corresponds to the functional classification of roadway segments, which is in accordance with the highway functional classification system by Federal Highway Administration (FHWA) (FHWA, 2013). This RCI variable has the following categories of roadway functional class (the two-digit codes are the RCI codes):

- 01 - Rural principal arterial - interstate

- 02 - Rural principal arterial - other

- 06 - Rural minor arterial

- 07 - Rural major collector

- $\quad 08$ - Rural minor collector

- 09 - Rural local

- $\quad 11$ - Urban principal arterial-interstate

- 12 - Urban principal arterial-other freeways and expressways

- 14 - Urban principal arterial-other 
- 16 - Urban minor arterial

- 17 - Urban collector

- 19 - Urban local

Other urban principal arterials (code "14") and minor arterials (code "16") fall into the category of urban and suburban arterial segments.

The TYPEROAD feature class in the RCI determines whether a roadway segment is divided or undivided. This variable defines roadway type by the following categories (the one-digit codes are the RCI codes):

- 0 - Not divided

- 2 - Divided (painted or physical)

- 4 - One-way

Segments are undivided if roadway type is coded as " 0 " and are divided if roadway type is coded as " 2 ". The HSM does not provide guidelines for one-way streets. Therefore, one-way streets were excluded from further analysis.

The next step was to separate the undivided and divided urban and suburban arterials by number of through lanes and type of median. The NOLANES variable in the RCI represents the number of through lanes in a segment and the RDMEDIAN variable determines the type of median, if present. The number of lanes is assigned in the RCI database depending on whether a segment is undivided or divided. For undivided segments, the number of lanes with digit " 2 " indicates two lanes in both directions. On the other hand, the number of lanes with digit " 2 " for divided segments indicates two lanes in each direction. The presence of a center TWLTL can be identified by the painted TWLTL median type in the RCI. Three-lane and five-lane arterials with a center TWLTL 
were, therefore, identified as divided segments that have median type code " 01 " and the number of lanes with values 1 and 2, respectively.

\subsubsection{Generation of Homogeneous Segments}

For each of the five urban and suburban roadway segment types, a dynamic segmentation process was carried out to generate homogeneous segments. A homogeneous segment has uniform roadway geometry, traffic volume, and traffic control features. Figure 3-1 provides an example demonstrating how a roadway segment is divided by roadway design features (e.g., lane width, median width, and number of lanes) to create homogeneous segments. AADT, median width, and posted speed limit were used to divide each type of urban and suburban arterials into homogeneous segments.

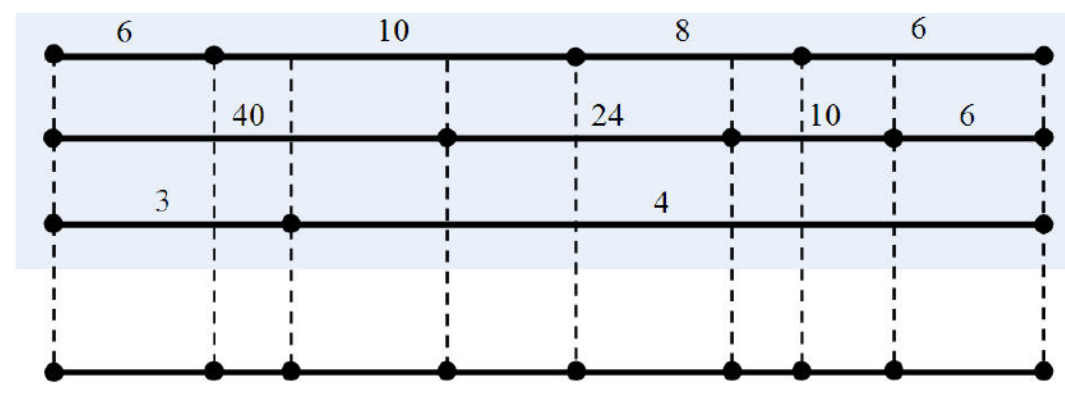

Lane width (ft)

Median width (ft)

Number of lanes

Homogeneous segments

\section{FIGURE 3-1 Illustration of Homogeneous Segments (Lu, 2013)}

A description of how the data variables were retrieved from the RCI and processed for the purpose of segmentation is provided herein. The SECTADT variable in the RCI provides the AADT data. Eight years (i.e., 2005 to 2012) of AADT data were retrieved from the corresponding year's RCI database and used in the segmentation process. The MAXSPEED RCI feature provides posted speed limit of a segment. Based on the values of the MAXSPEED variable, speed limits were categorized into two groups: (1) less than or equal to $30 \mathrm{mph}$, and (2) greater than $30 \mathrm{mph}$. The MEDWIDTH 
variable in the RCI provides information on median width. Note that median width is recorded to the nearest foot in the RCI database, whereas the HSM suggests rounding the median width to the nearest $10 \mathrm{ft}$. Therefore, the following adjustments were made prior to segmentation:

- $1 \mathrm{ft}$ to $14 \mathrm{ft}$ was rounded to $10 \mathrm{ft}$,

- $15 \mathrm{ft}$ to $24 \mathrm{ft}$ was rounded to $20 \mathrm{ft}$,

- $25 \mathrm{ft}$ to $34 \mathrm{ft}$ was rounded to $30 \mathrm{ft}$,

- $35 \mathrm{ft}$ to $44 \mathrm{ft}$ was rounded to $40 \mathrm{ft}$,

- $45 \mathrm{ft}$ to $54 \mathrm{ft}$ was rounded to $50 \mathrm{ft}$,

- $55 \mathrm{ft}$ to $64 \mathrm{ft}$ was rounded to $60 \mathrm{ft}$,

- $65 \mathrm{ft}$ to $74 \mathrm{ft}$ was rounded to $70 \mathrm{ft}$,

- $75 \mathrm{ft}$ to $84 \mathrm{ft}$ was rounded to $80 \mathrm{ft}$,

- $85 \mathrm{ft}$ to $94 \mathrm{ft}$ was rounded to $90 \mathrm{ft}$, and

- $95 \mathrm{ft}$ or more was rounded to $100 \mathrm{ft}$.

The HSM does not recommend any minimum segment length for data processing. Based on the other states' practices on the selection of minimum segment length (Srinivasan et al., 2011 and Dixon et al., 2012), segments shorter than 0.04 miles were not considered in the analysis.

\subsubsection{Data Collection}

The next step after generating homogeneous segments was to retrieve information for the variables identified in the HSM. Despite being an archive of more than 100 roadway data elements, most of the variables used in calibrating the HSM predictive models are not available in the RCI. As such, a major effort was undertaken to collect 
data for the variables that are not available in the RCI. To facilitate the data collection process, an in-house web-based application called Visual Roadway Inventory Collection System (VRICS) was customized. The application works as follows. It first reads a linear-referenced roadway segment shape file, converts its coordinates to the Google Maps projection, and then displays the segment in the system pinpointing the beginning and end mileposts. Figure 3-2 shows a screen capture of the main interface of the application. Similar to a typical video $\log$ system, VRICS provides the option to go forward and backward at a certain speed along the segment for smooth observation of roadway features using either Street view or Satellite view. An important component of the application is that it includes an input pane that allows the user to quickly specify and record the observed data. Data for categorical variables were recorded by selecting from a number of options provided in a drop-down list while data for continuous variables were entered directly in the box next to them. 


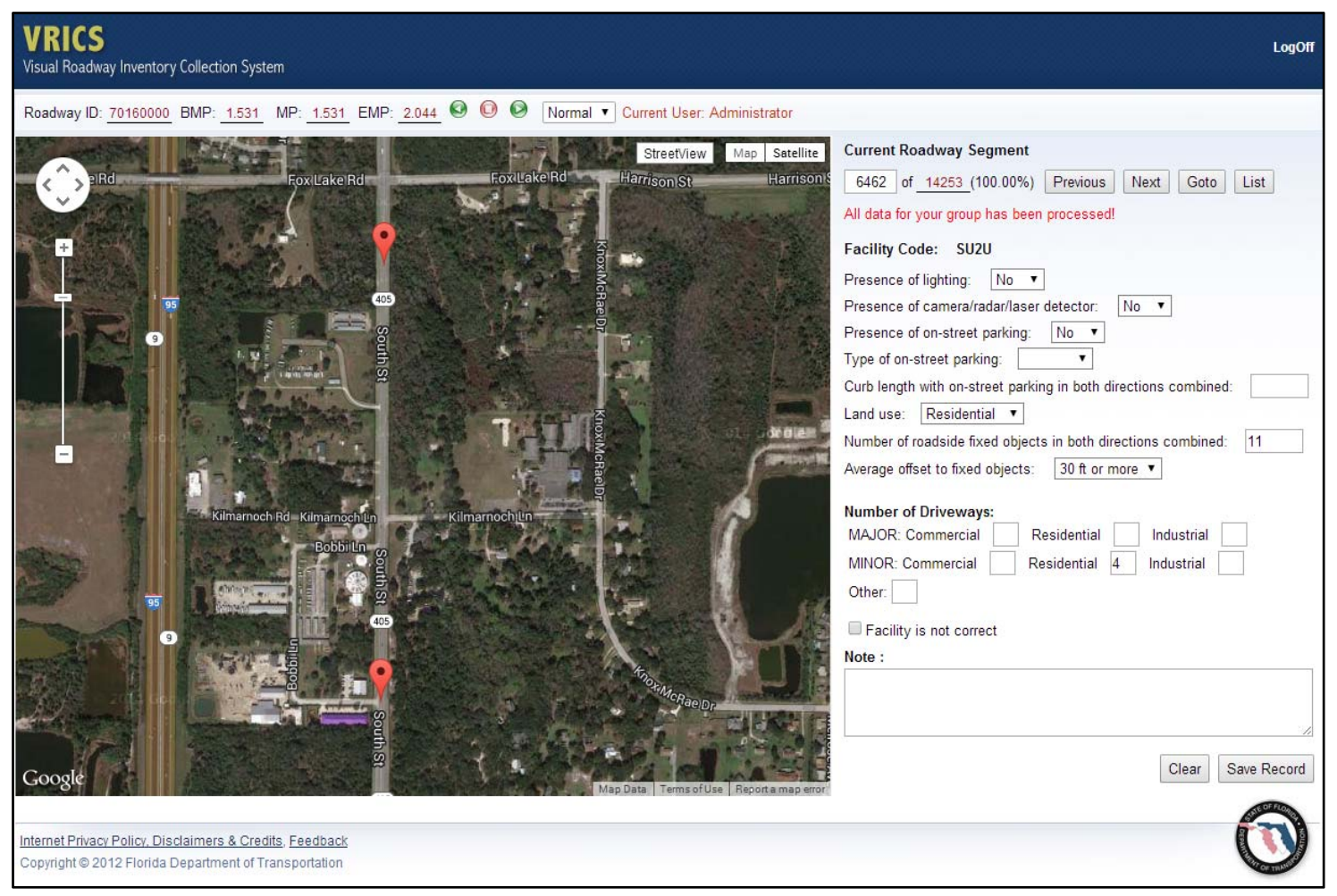

FIGURE 3-2 VRICS Application for Segment Data Collection

The variables for which data were collected for segments and the data collection process using VRICS are described below:

- Presence of lighting: Two options, "yes" or "no", were provided to collect information on presence of lighting. If lighting poles were present throughout a segment, "yes" was selected to indicate presence of lighting; otherwise, "no" was selected indicating absence of lighting along the segment. Since the minimum spacing between light poles is not specified in the HSM, subjective judgment was used to collect this information.

- Presence of automated speed enforcement: Two options, "yes" or "no", were provided to collect information on presence of automated speed enforcement. If any speeding enforcement device was found at any point along the segment, "yes" 
was selected to indicate presence of automated speed enforcement; otherwise, "no" was selected indicating absence of automated speed enforcement in the segment.

- Presence of on-street parking: Two options, "yes" or "no", were provided to collect information on presence of on-street parking. If there was delineation of designated parking space(s) along the segment on any side of the roadway, "yes" was selected to indicate presence of on-street parking; otherwise, "no" was selected indicating absence of on-street parking.

- Type of on-street parking: Two options, "angle" or "parallel", were provided to collect information on type of on-street parking. The specific type was selected based on the orientation of the designated parking space(s), which could easily be discernible from the pavement markings.

- Curb length with on-street parking: The length of on-street parking spaces on both sides of the roadway segment was measured by counting the number of parking spaces and then multiplying the number by $20 \mathrm{ft}$, which typically represents the length of a parking space for a standard vehicle.

- Land use: This variable is only applicable to the segments with on-street parking facilities. The most appropriate land use type for a segment was recorded from one of the following options: commercial, industrial, institutional, residential, or other.

- Number of driveways by land-use type: Driveways on both sides of the roadway segment were counted and recorded for the following seven driveway types: major commercial driveways, major residential driveways, major 
industrial/institutional driveways, minor commercial driveways, minor residential driveways, minor industrial/institutional driveways, and other driveways. The HSM defines major driveways as those that provide access to a minimum of 50 parking spaces and minor driveways as those that provide access to fewer than 50 parking spaces. A driveway, whether major or minor, could be identified from aerial images. At times, when it was difficult to count the number of parking spaces due to obstructed vision, subjective judgment was used to identify driveway type. In addition, the following suggestions in the HSM were adopted to determine whether a driveway serves commercial, residential, or industrial land use (AASHTO, 2010):

"Commercial driveways provide access to establishments that serve retail customers. Residential driveways serve single- and multiple-family dwellings. Industrial/institutional driveways serve factories, warehouses, schools, hospitals, churches, offices, public facilities, and other places of employment. Commercial sites with no restriction on access along an entire property frontage are generally counted as two driveways." Driveways that did not fall into any of the above six types were recorded as other driveways.

- Number of roadside fixed objects: Fixed objects such as signs, poles, trees, fences etc. were counted on both sides of the roadway segment. As recommended in the HSM, the following exceptions were made while counting the roadside fixed objects:

- Objects located close to each other such as within $70 \mathrm{ft}$ were counted as one object. 
- Continuous objects for every $70 \mathrm{ft}$ of length along the longitudinal direction of the roadway were counted as one object.

- Minute objects, i.e., objects likely smaller than 4 inches in diameter, were not counted.

- Objects located in the median on divided arterials were not counted as roadside objects.

- Offset to roadside fixed object: For each roadway segment, an average distance from the edge of the roadway to roadside objects was determined and input into one of the following categories: $2 \mathrm{ft}, 5 \mathrm{ft}, 10 \mathrm{ft}, 15 \mathrm{ft}, 20 \mathrm{ft}, 25 \mathrm{ft}$, and $\geq 30 \mathrm{ft}$.

\subsection{Intersection Data}

FDOT currently maintains a database of all nodes on the SHS in RDWTBL 25 (i.e., Roadway Table 25). However, it was not possible to accurately extract three-leg and four-leg signalized and unsignalized intersections. Therefore, alternative approaches to populate the intersection data set were explored. Several Geographic Information System (GIS) shape files available in the Transportation Statistics Office website were used to extract intersection data. The AADT GIS layer was used to extract all the intersections for which AADT data were available. Next, the State Roads GIS layer was overlaid on the extracted intersections layer to retrieve intersections on state roads alone. Finally, Signalized Intersections GIS layer was overlaid to identify signalized intersections. All the remaining intersections in the extracted intersections layer that were not identified as signalized intersections were recorded as unsignalized intersections. A total of 1,038 intersections were identified as signalized intersections and a total of 1,555 intersections were identified as unsignalized intersections using this approach. The 
URBSIZE variable in the RCI for the major approach roadway ID was used to determine whether the intersection is in rural or urban area.

Once the intersections were spatially identified, VRICS was used to collect information for the variables pertaining to intersections. Figure 3-3 shows the screen capture of the VRICS application customized to collect intersection data. The VRICS system also included a circle with a radius of $1,000 \mathrm{ft}$ around every intersection to facilitate data collection. Note that some variables are specific to the overall intersection, while some variables are specific to the approach of the intersection. The variables for which data were collected for intersections and the data collection process using VRICS are described below. 


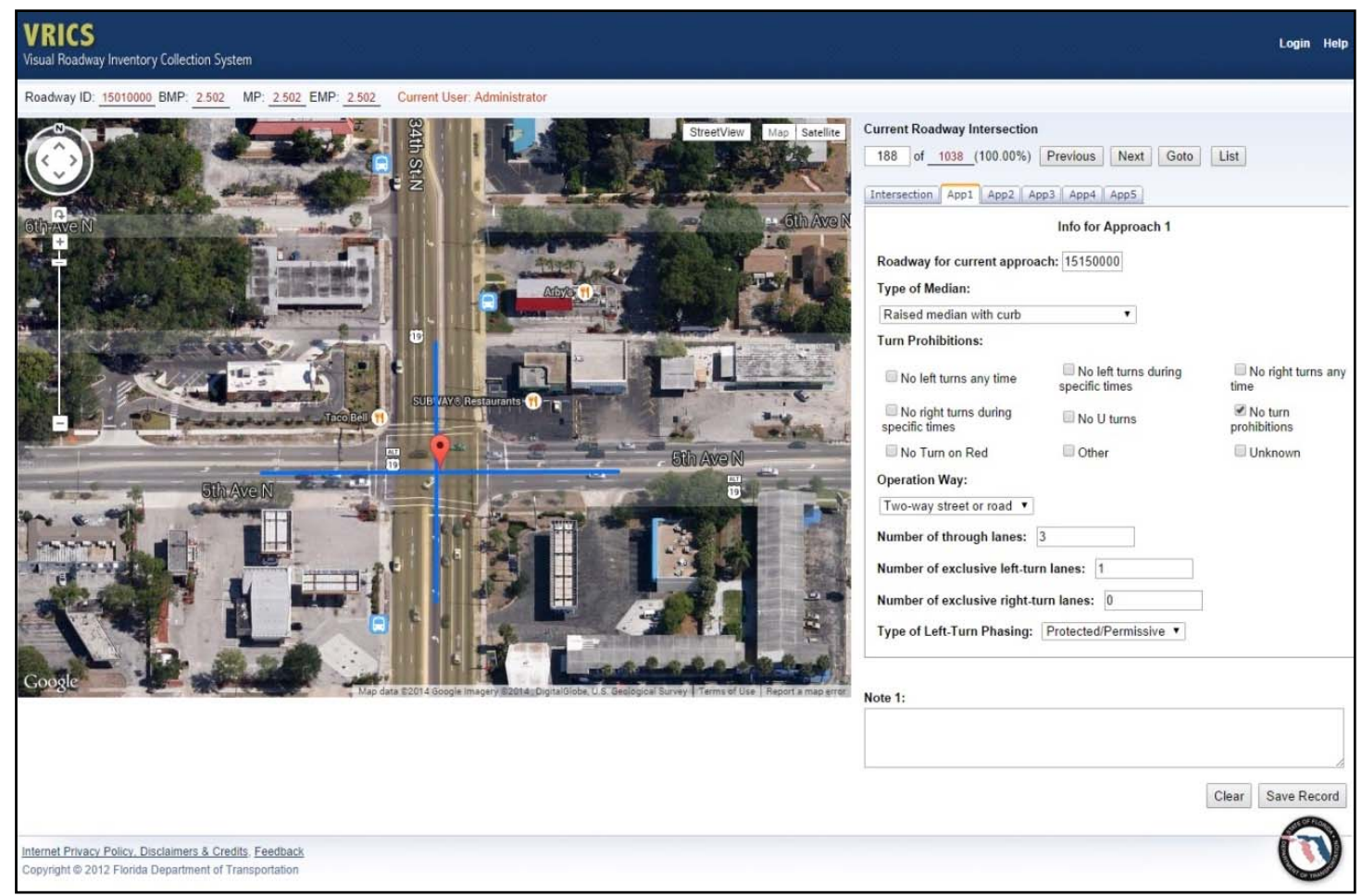

(a) Input Pane Showing Variables Specific to Overall Intersection

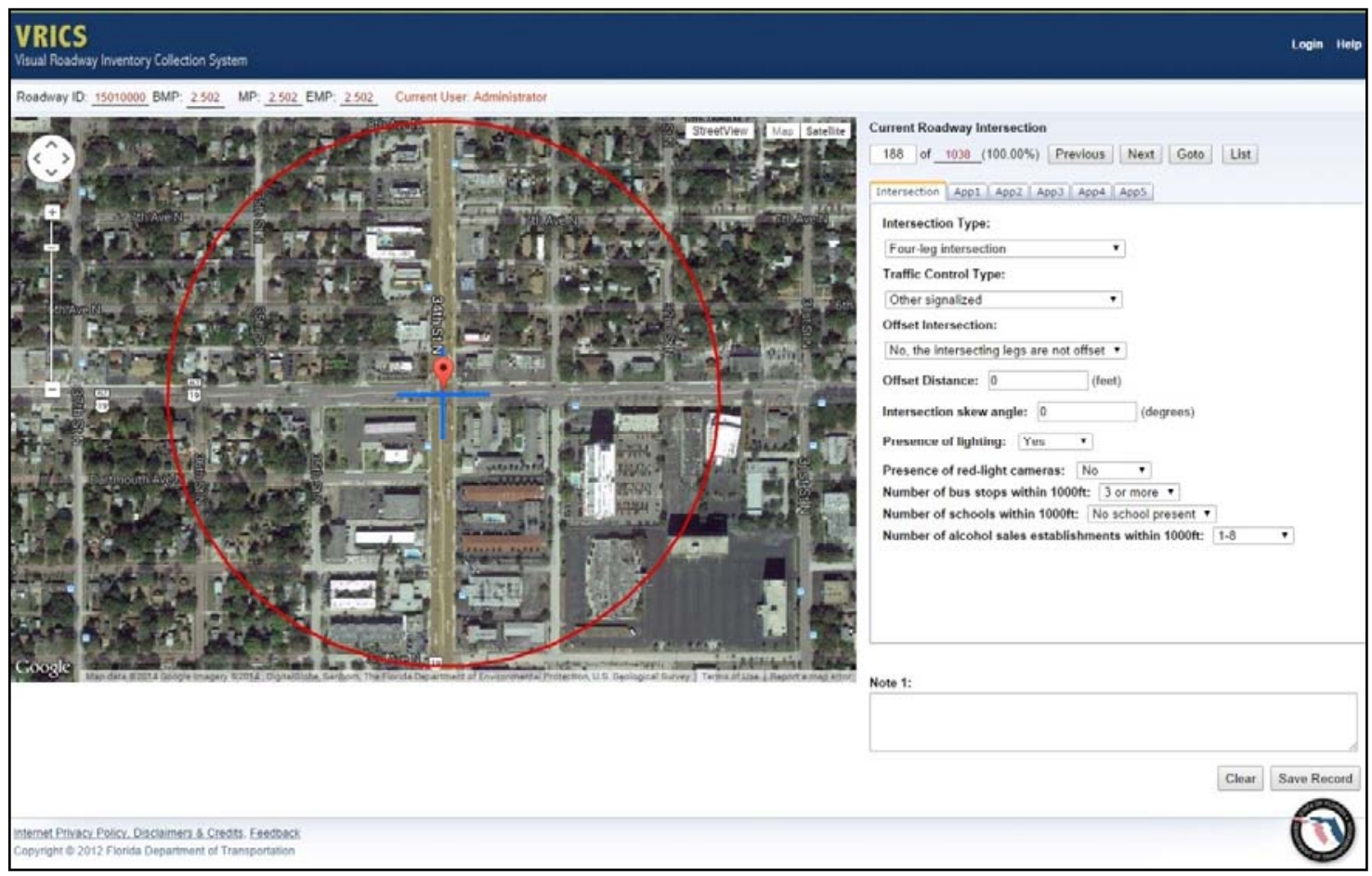

(b) Input Pane Showing Variables Specific to Intersection Approach FIGURE 3-3 VRICS Application for Intersection Data Collection 
- Type of intersection: The intersection type was selected from the following list:

- Four-leg intersection

- Tee-intersection

- Y-intersection

- Traffic circle or roundabout

- Multi-leg (5 or more) intersections

- Others

Note that only four-leg and three-leg intersections (Tee- and Y- intersections) were included in the analysis.

- Type of traffic control: The prevailing traffic control type was selected from the following list:

- No control

- Stop signs on cross street only

- Stop signs on mainline only

- All-way stop signs

- Two-way flasher (red on cross-street)

- Two-way flasher (red on mainline)

- All-way flasher (red on all approaches)

- Yield signs on cross street only

- Yield signs on mainline only

- Other non-signalized

- Other signalized

- Roundabout 
- Others (unknown)

Based on the recommendations in the HSM, intersections with "stop signs on cross street only" control were considered as stop-controlled intersections and intersections with "other signalized" control were considered as signalized intersections.

- Presence of lighting: Two options, "yes" or "no", were provided to record information on presence of lighting. If lighting poles were observed at the intersection, "yes" was selected indicating presence of lighting; otherwise, "no" was selected indicating absence of lighting.

- Presence of red-light cameras: Two options, "yes" or "no", were provided to collect information on presence of red-light cameras. If red-light cameras were installed on any of the signal mast arms or if "Red Light Photo Enforced" sign was mounted on poles upstream of the intersection, "yes" was selected indicating presence of red-light cameras; otherwise, "no" was selected indicating absence of red-light cameras.

- Number of bus stops within 1,000 ft of intersection: The number of bus stops within $1,000 \mathrm{ft}$ of the intersection was counted by observing bus stop signs in aerial view. The information was then recorded by selecting one of the following three options: "0" if there are no bus stops; "1-2" if there are one or two bus stops; and " $\geq 3$ " if there are more than two bus stops.

- Presence of schools within 1,000 ft of intersection: The information on presence of school could be obtained from the school name(s) provided on aerial images in 
Google Maps. If there is any school within 1,000 ft of the intersection "yes" was selected; otherwise, "no" was selected.

- Number of alcohol sales establishments within 1,000 ft of intersection: Bars, restaurants, pharmacies, grocery stores, convenience stores, and liquor stores anywhere within $1,000 \mathrm{ft}$ of the intersection were considered as the alcohol sales establishments, and counted. The information was then recorded by selecting one of the following three options: "0" if there are no alcohol sales establishments; " 1 8 " if there are at least eight alcohol sales establishments; and " $\geq 9$ " if there are more than eight alcohol sales establishments.

- Intersection left-turn lanes: For each approach, the number of exclusive left-turn lanes was counted and recorded.

- Intersection right-turn lanes: For each approach, the number of exclusive rightturn lanes was counted and recorded.

- Type of left-turn phasing: The information on type of left-turn signal phasing pertains to signalized intersections. For an approach, the type of left-turn phasing (i.e., permissive, protected/permissive, or protected) was determined from the number of left-turn lanes and the signal head assembly.

- Use of right-turn-on-red (RTOR) signal operation: The information on prohibition of RTOR pertains to signalized intersections. For each approach, if a sign saying "No Right Turn On Red" was placed either on the signal mast arm or several feet upstream of the approach, "yes" was selected indicating prohibited operation of RTOR; otherwise, "no" was selected indicating permission of RTOR. 
- Maximum number of lanes crossed by pedestrians: For each approach, the total number of lanes including through lanes, left-turn lanes, and right-turn lanes on both directions was counted and recorded. The highest total number of lanes in any approach was determined as the maximum number of lanes crossed by pedestrians.

- Pedestrian activity level: The HSM identifies the following five levels of pedestrian activity by daily pedestrian crossing volumes: high, medium-high, medium, medium-low, and low. Because of limited pedestrian exposure data, medium pedestrian activity level was assumed for all the locations.

\subsection{Traffic Data}

The HSM identifies AADT as the most critical variable in predicting crashes. As such, the manual requires AADT data for segments and for both major and minor road approaches of intersections in applying the SPFs to predict segment and intersection crashes, respectively. AADT data for the entire study period (2005-2012) were retrieved from the corresponding year's RCI database based on roadway ID and milepost information. As aforementioned, the SECTADT variable in the RCI provides AADT data.

\subsection{Crash Data}

Eight years of crash data from 2005 to 2012 were retrieved from FDOT's Unified Basemap Repository (UBR) system. Note that only crashes that occurred on the state roads were included in the analysis. The procedures followed to assign crashes to segments and intersections are discussed below. 


\subsubsection{Crash Assignment to Segments}

The SITELOCA variable in the UBR was used to retrieve the location of crashes. The variable uses the following two-digit codes to determine the facilities where the crashes occurred (FDOT, 2013):

- 00 - Unknown

- 01 - Not at intersection, railroad crossing, or bridge

- $02-$ At intersection

- 03 - Influenced by intersection

- 04 - Driveway access

- 05 - Railroad

- 06 - Bridge

- 07 - Entrance ramp

- 08 - Exit ramp

- 09 - Private parking lot

- 10 - Public parking lot

- 11 - Private property

- 12 - Toll booth

- 13 - Public bus stop zone

- $\quad 77$ - All other (explained in narrative)

Crashes that occurred at intersections (code "02”), within intersection influence areas (code “03"), and at entrance or exit ramps (codes “07" and "08") were not considered as segment-related crashes. The remaining crashes were assigned to the 
segments based on roadway ID and the milepost at which the crash occurred. Crashes that occurred on the intersecting point between two roadway segments were consistently assigned to the beginning segment. The number and types of crashes in each segment were counted using structured query language (SQL), implemented in the sqldf package of the statistical software $\boldsymbol{R}$ (R core team, 2014).

\subsubsection{Crash Assignment to Intersections}

Crashes that occurred within $250 \mathrm{ft}$ from the center of intersection are typically considered as intersection-related crashes (AASHTO, 2010). The spatial tool in ArcGIS was used to identify intersection-related crashes. First, an intersection location data layer was imported into ArcGIS. A new layer was then created by drawing a 250 -ft buffer around each intersection. The shape file thus formed was merged with crash data shape files of each study year in ArcGIS. The output of the merged file comprised intersection characteristics data and associated crash records at intersections and on all approaches within $250 \mathrm{ft}$ from the center of the intersections. Crashes observed in the overlapping portion of two or more buffers were assigned to the nearest intersection. The process was

repeated for each year of crash data. Similar to segments, the number and types of crashes in each intersection were counted using SQL, implemented in the sqldf package of the statistical software $\boldsymbol{R}$ (R core team, 2014).

\subsection{Data Preparation}

In this step, the roadway geometry, traffic, and crash data were thoroughly checked for possible outliers and inconsistencies. Several considerations were made while preparing the final data set, including: 
- Sites identified with any type of construction work were not included in the analysis.

- Intersections where any of the approaches were observed to carry one-directional traffic were not included in the analysis.

- Sites with extremely high or extremely low AADT values were considered as outliers and, therefore, were excluded from the analysis.

- Individual sites with a huge difference in AADT between consecutive years were also excluded from the analysis.

After making these adjustments, the final data set was created by merging site characteristics data, crash data, and traffic data for each roadway segment and intersection type. Tables 3-2 and 3-3 give summary statistics of the roadway segment and intersection types considered in this study, respectively.

TABLE 3-2 Summary Statistics of Urban and Suburban Arterial Segments

\begin{tabular}{|l|c|c|c|c|}
\hline Segment Type & $\begin{array}{c}\text { Number of } \\
\text { Segments }\end{array}$ & $\begin{array}{c}\text { Total } \\
\text { Length } \\
\text { (miles) }\end{array}$ & $\begin{array}{c}\text { Total } \\
\text { Number } \\
\text { of Crashes } \\
\text { (2005-2012) }\end{array}$ & $\begin{array}{c}\text { Number of } \\
\text { Crashes per } \\
\text { Mile per Year }\end{array}$ \\
\hline Two-lane undivided & 1,791 & 616.7 & 9,453 & 1.92 \\
\hline Three-lane with a TWLTL & 359 & 67.0 & 1,769 & 3.30 \\
\hline Four-lane undivided & 266 & 52.2 & 1,900 & 4.55 \\
\hline Four-lane divided & 4,969 & 1400.7 & 48,151 & 4.30 \\
\hline Five-lane with a TWLTL & 1,084 & 269.2 & 12,367 & 5.74 \\
\hline
\end{tabular}

TABLE 3-3 Summary Statistics of Urban and Suburban Intersections

\begin{tabular}{|l|c|c|c|}
\hline Intersection Type & $\begin{array}{c}\text { Number of } \\
\text { Intersections }\end{array}$ & $\begin{array}{c}\text { Total Number } \\
\text { of Crashes } \\
\text { (2005-2012) }\end{array}$ & $\begin{array}{c}\text { Number of } \\
\text { Crashes per Year } \\
\text { per Intersection }\end{array}$ \\
\hline Three-leg stop-controlled & 317 & 4,588 & 1.81 \\
\hline Four-leg signalized & 397 & 54,496 & 17.16 \\
\hline
\end{tabular}




\subsection{Summary}

This chapter presented the data collection efforts undertaken in this research with regard to the data needs in the HSM. The RCI database, the UBR database, RDWTBL 25, AADT GIS layer, state roads GIS layer, and signalized intersections GIS layer were used to extract the data variables. Traffic volume and crash records for a total of eight years (2005-2012) were extracted from the RCI and the UBR databases. However, several variables pertaining to roadway geometry and intersection characteristics are currently unavailable in the FDOT databases. Information for those variables was collected using aerial images. Note that data collection was limited to state roads in urban and suburban areas. Tables A-1 through A-7 in Appendix A provide detailed descriptive statistics of data variables for each segment and intersection type. 


\section{CHAPTER 4}

\section{METHODOLOGY}

This chapter describes the methodological processes applied in this dissertation research to accomplish the stated objectives. The first section gives an overview of the entire methodology. A detailed description of the procedures is then presented in the subsequent sections.

\subsection{Methodological Framework}

Figure 4-1 presents the methodological framework representing the processes carried out to accomplish the objectives of this research. All the processes were based on the data set constituting roadway geometry, traffic, and crash data. As noted in Chapter 3, the final data set was built for roadway segments and intersections for the urban and suburban facilities in Florida.

First, the complete data set was used to determine the calibration factors. A sensitivity analysis was then conducted to evaluate the sample size criteria recommended in the HSM. In the sensitivity analysis, a number of samples were randomly generated with different sample sizes and calibration factors were again calculated for each sample size. Based on the sensitivity between calibration factors for various sample sizes, a reliable minimum sample size was recommended for calibrating the HSM predictive models for urban and suburban roadway segment and intersection types.

Next, several paired t-tests were performed to determine whether or not there is any statistically significant difference in calibration factors computed between every three years, every two years, or every year. The minimum sample size determined in the 
previous step was used to derive calibration factors and validate the recommended intervals (.i.e., one to three years) in the HSM for updating the calibration factor for each site type.

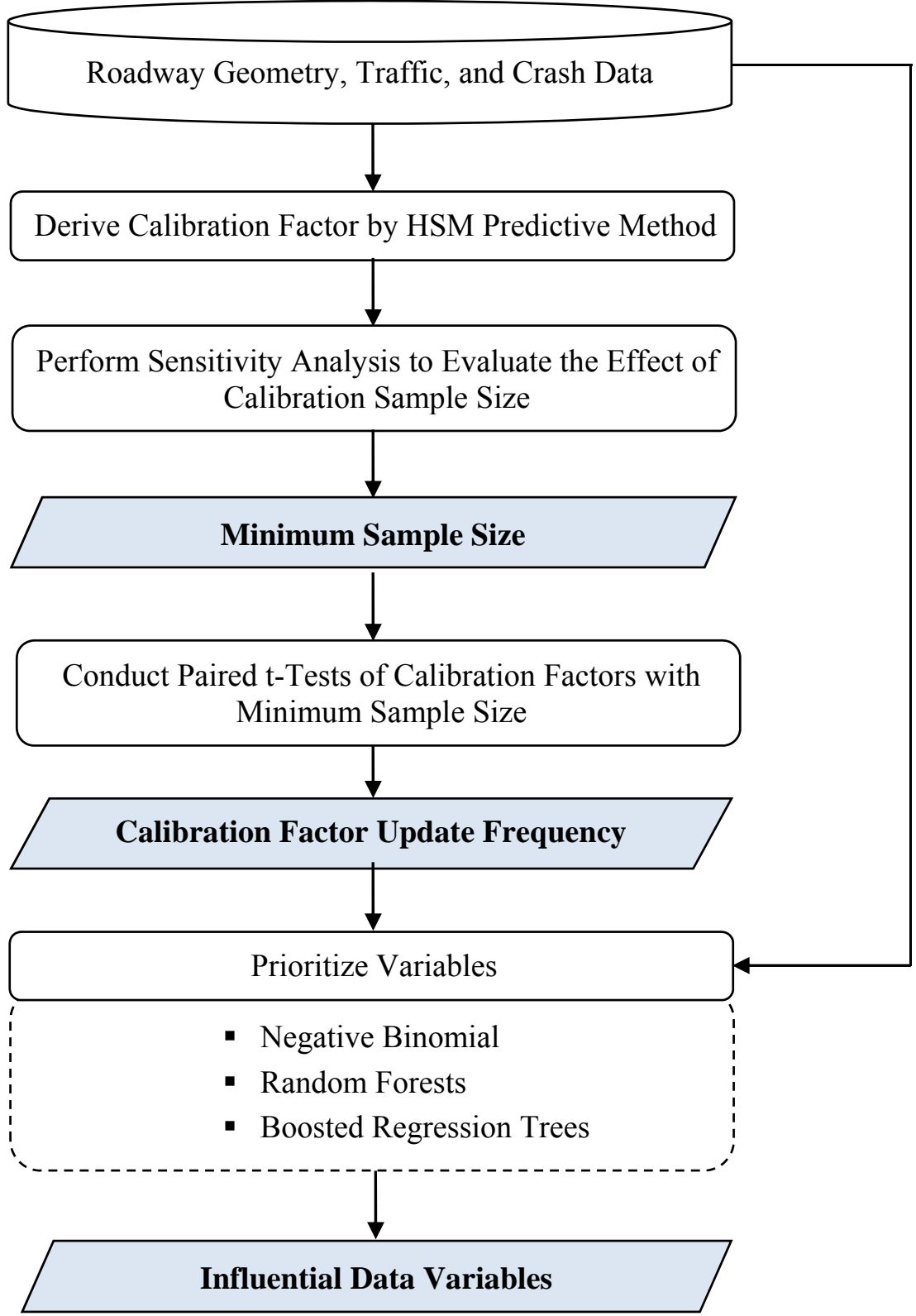

FIGURE 4-1 Methodological Framework 
In order to achieve the third objective, i.e., to identify and prioritize influential variables for calibration process, variable importance was determined. Three methods including negative binomial (NB), random forests (RF), and boosted regression trees (BRT) were used to rank and prioritize the HSM variables based on their effect on crash predictions. The following sections discuss in detail the methodological steps presented in Figure 4-1.

\subsection{Calibration Factor Estimation}

The calibration factor for a particular site type is defined as the ratio of the total number of observed crashes to the total number of predicted crashes. Thus, calibration factor $\left(C_{x}\right)$ can be expressed as follows (AASHTO, 2010):

$$
C_{x}=\frac{\sum_{\text {All Sites }} \text { Observed Crashes }}{\sum_{\text {All Sites }} \text { Predicted Crashes }}
$$

In this research, the observed crashes were retrieved from the UBR crash database and the number of predicted crashes was determined using the HSM predictive method for urban and suburban arterial segments and intersections. The procedure to determine the predicted number of crashes is briefly explained in the following subsections for urban and suburban arterial segments and intersections, respectively.

\subsubsection{Segments}

The predictive models in the HSM for urban and suburban arterial segments are based on the following equations:

$$
\begin{gathered}
N_{s p f r s}=N_{b r m v}+N_{b r s v}+N_{b r d w y} \\
N_{b r}=N_{s p f r s} \times\left(C M F_{1 r} \times C M F_{2 r} \times \ldots \times C M F_{n r}\right) \\
N_{\text {predicted } r s}=N_{b r}+N_{\text {pedr }}+N_{\text {biker }}
\end{gathered}
$$


where,

$$
\begin{aligned}
& N_{\text {predicted } r s}=\text { predicted average crash frequency of an individual roadway } \\
& N_{b r} \quad=\text { predicted average crash frequency of an individual roadway } \\
& \text { segment (excluding vehicle-pedestrian and vehicle-bicycle } \\
& \text { crashes), } \\
& N_{s p f r s} \quad=\text { predicted average crash frequency of an individual roadway } \\
& \text { segment for base conditions (excluding vehicle-pedestrian and } \\
& \text { vehicle-bicycle crashes), } \\
& N_{\text {pedr }} \quad=\text { predicted average crash frequency of vehicle-pedestrian crashes, } \\
& N_{\text {biker }}=\text { predicted average crash frequency of vehicle-bicycle crashes, } \\
& N_{b r m v} \quad=\text { predicted average crash frequency of multiple-vehicle non- } \\
& \text { driveway crashes for base conditions, } \\
& N_{\text {brsv }} \quad=\text { predicted average crash frequency of single-vehicle crashes for } \\
& \text { base conditions, } \\
& N_{b r d w y} \quad=\text { predicted average crash frequency of multiple-vehicle driveway- } \\
& \text { related crashes for base conditions, } \\
& C M F_{1 r}, \ldots, C M F_{n r} \quad=\text { crash modification factors for each of the } n \text { geometric conditions } \\
& \text { of an individual roadway segment. }
\end{aligned}
$$

The SPF components of the predictive models in Equations (4-2) through (4-4) correspond to five crash types including multiple-vehicle non-driveway crashes $\left(N_{b r m v}\right)$, single-vehicle crashes $\left(N_{b r s v}\right)$, multiple-vehicle driveway-related crashes $\left(N_{b r d w y}\right)$, 
vehicle-pedestrian crashes $\left(N_{\text {pedr }}\right)$, and vehicle-bicycle crashes $\left(N_{\text {biker }}\right)$. The base conditions for the SPFs are as follows:

- no on-street parking,

- no roadside fixed objects,

- median width of $15 \mathrm{ft}$,

- no lighting, and

- no automated speed enforcement.

First, $N_{b r m v}, N_{b r s v}$, and $N_{b r d w y}$ were estimated using Equations (4-5) through (4-7), respectively, as shown below:

$$
\begin{gathered}
N_{\text {brmv }}=\exp \left(\alpha_{0}+\alpha_{1} \times \ln (A A D T)+\ln (L)\right) \\
N_{\text {brsv }}=\exp \left(\beta_{0}+\beta_{1} \times \ln (A A D T)+\ln (L)\right) \\
N_{\text {brdwy }}=\sum_{\substack{\text { All Driveway } \\
\text { Types }}} n_{j} \times N_{j} \times\left(\frac{A A D T}{15,000}\right)^{t}
\end{gathered}
$$

where,

$$
\begin{aligned}
A A D T= & \text { annual average daily traffic volume on roadway segment (vehicles per } \\
& \text { day) } \\
L= & \text { length of roadway segment (miles), } \\
\alpha_{0}, \alpha_{1}= & \text { coefficients of SPF for multiple-vehicle non-driveway crashes (values } \\
& \text { are given in Table B-1 in Appendix B), } \\
\beta_{0}, \beta_{1}= & \text { coefficients of SPF for single-vehicle crashes (values are given in Table } \\
& \text { B-2 in Appendix B), }
\end{aligned}
$$




$$
\begin{aligned}
n_{j}= & \text { number of driveways within roadway segment of driveway type } j \\
& \text { including all driveways on both sides of the road, } \\
N_{j}= & \text { number of driveway-related crashes per driveway per year for driveway } \\
& \text { type } j \text { (values are given in Table B-3 in Appendix B), and } \\
= & \text { coefficient of traffic volume adjustment (values are given in Table B-3 } \\
& \text { in Appendix B). }
\end{aligned}
$$

Next, the sum of $N_{b r m v}, N_{b r s v}$, and $N_{b r d w y}$ was calculated to obtain $N_{s p f r s}$, which was then multiplied with CMFs to account for the effects of prevailing (i.e., non-base) conditions on predicted crash frequency of roadway segments. A total of five CMFs were applied, as described below $\left(C M F_{1 r}\right.$ through $\left.C M F_{5 r}\right)$ :

\section{$C M F_{1 r}-$ On-street Parking}

The base condition for $C M F_{1 r}$ is the absence of on-street parking on a roadway segment (i.e., $C M F_{1 r}=1.00$ ). The effect of presence of on-street parking on crash predictions was determined as:

$$
C M F_{1 r}=1.0+p_{p k} \times\left(f_{p k}-1.0\right)
$$

where,

$$
\begin{aligned}
f_{p k}= & \text { factor for on-street parking (values are given in Table B-4 in Appendix B), } \\
p_{p k}= & \text { proportion of curb length with on-street parking measured as }\left(0.5 \times \frac{L_{p k}}{L}\right), \\
L_{p k}= & \text { sum of curb length with on-street parking combined for both sides of the } \\
& \quad \text { road (miles), and } \\
L= & \text { length of roadway segment (miles). }
\end{aligned}
$$




\section{$\mathrm{CMF}_{2 r}-$ Roadside Fixed Objects}

The base condition for $C M F_{2 r}$ is the absence of roadside objects on a roadway segment (i.e., $C M F_{2 r}=1.00$ ). The effect of presence of roadside fixed objects on crash predictions was determined as follows:

$$
C M F_{2 r}=f_{\text {offset }} \times D_{f o} \times p_{f o}+\left(1.0-p_{f o}\right)
$$

where,

$$
\begin{aligned}
f_{o f f s e t}= & \text { fixed-object offset factor (values are given in Table B-5 in Appendix B), } \\
D_{f o}= & \text { fixed-object density for both sides of the road combined (number of } \\
& \text { fixed objects per mile), and } \\
p_{f o} \quad= & \text { fixed-object crashes as a proportion of total crashes. }
\end{aligned}
$$

When the $C M F_{2 r}$ was less than 1.00 (for sites with low density of roadside fixed objects), a minimum value of 1.00 was assigned to $C M F_{2 r}$.

$C M F_{3 r}-$ Median Width

This CMF for median width is applicable only to four-lane divided arterial segments. The base condition is a median width of $15 \mathrm{ft}$ (i.e., $C M F_{3 r}=1.00$ ). The value of $C M F_{3 r}$ for medians wider than $15 \mathrm{ft}$ is less than 1.00 and for medians narrower than 15 $\mathrm{ft}$, it is greater than 1.00. Table B-6 in Appendix B provides the value of $C M F_{3 r}$ for the effect of different median widths.

\section{$C M F_{4 r}-$ Lighting}

The base condition for $C M F_{4 r}$ is the absence of lighting along the roadway segment (i.e., $C M F_{4 r}=1.00$ ). The effect of lighting on crash predictions was determined 
as follows:

$$
C M F_{4 r}=1.0-\left(p_{n r} \times\left(1.0-0.72 \times p_{i n r}-0.83 \times p_{p n r}\right)\right)
$$

where,

$$
\begin{aligned}
& p_{n r}=\text { proportion of total crashes for unlighted segments that occur at night, } \\
& p_{\text {inr }}=\text { proportion of total nighttime crashes for unlighted segments that involve } \\
& \quad \text { a fatality or injury, and } \\
& p_{p n r}=\quad \text { proportion of total nighttime crashes for unlighted segments that involve } \\
& \quad \text { PDO. }
\end{aligned}
$$

\section{$C M F_{5 r}-$ Automated Speed Enforcement}

The base condition for $C M F_{5 r}$ is the absence of automated speed enforcement on a roadway segment (i.e., $\left.C M F_{5 r}=1.00\right)$. A value of 0.95 was used for $C M F_{5 r}$ on segments where there was any camera, radar, or laser detector installed to detect speeding vehicles.

After determining $N_{b r}$, which is a product of $N_{s p f r s}$ and CMFs (i.e., $C M F_{1 r}$ through $C M F_{5 r}$ ), the other two SPF components (i.e., $N_{p e d r}$ and $N_{b i k e r}$ ) were calculated using Equations (4-11) and (4-12), as shown below:

$$
\begin{gathered}
N_{\text {pedr }}=N_{b r} \times f_{\text {pedr }} \\
N_{\text {biker }}=N_{b r} \times f_{\text {biker }}
\end{gathered}
$$

where,

$$
\begin{aligned}
f_{\text {pedr }}= & \text { pedestrian crash adjustment factor calculated as the ratio of total crashes } \\
& \text { (excluding vehicle-pedestrian and vehicle-bicycle crashes) to vehicle- } \\
& \text { pedestrian crashes for roadway segments, and }
\end{aligned}
$$


$f_{\text {biker }}=$ bicycle crash adjustment factor calculated as the ratio of total crashes (excluding vehicle-pedestrian and vehicle-bicycle crashes) to vehiclebicycle crashes for roadway segments.

\subsubsection{Intersections}

The predictive models in the HSM for urban and suburban arterial intersections are based on the following equations:

$$
\begin{gathered}
N_{\text {predicted int }}=N_{b i}+N_{\text {pedi }}+N_{\text {bikei }} \\
N_{b i}=N_{\text {spfint }} \times\left(C M F_{1 i} \times C M F_{2 i} \times \ldots \times C M F_{n i}\right) \\
N_{\text {spfint }}=N_{\text {bimv }}+N_{\text {bisv }}
\end{gathered}
$$

where,

$$
\begin{aligned}
N_{\text {predicted int }}= & \text { predicted average crash frequency at an intersection for the } \\
& \text { selected year, } \\
N_{\text {bi }} & \text { predicted average crash frequency at an intersection (excluding } \\
& \text { vehicle-pedestrian and vehicle-bicycle collisions) }, \\
N_{\text {spfint }} & \text { predicted average crash frequency of intersection-related } \\
& \text { crashes for base conditions (excluding vehicle-pedestrian and } \\
& \text { vehicle-bicycle crashes) }, \\
= & \text { predicted average crash frequency of vehicle-pedestrian crashes } \\
& \text { for an intersection, } \\
N_{\text {pedi }} & \text { predicted average crash frequency of vehicle-bicycle crashes for } \\
& \text { an intersection, }
\end{aligned}
$$




$$
\begin{aligned}
N_{\text {bimv }} \quad & \text { predicted average crash frequency of multiple-vehicle crashes } \\
& \text { for base conditions } \\
N_{\text {bisv }} & \text { predicted average crash frequency of single-vehicle crashes for } \\
& \text { base conditions, and } \\
C M F_{1 i}, \ldots, C M F_{n i}= & \text { crash modification factors for each of the } n \text { geometric } \\
& \text { conditions or traffic control features of an intersection. }
\end{aligned}
$$

The SPF components of the predictive models in Equations (4-13) through (4-15) correspond to four crash types including multiple-vehicle crashes $\left(N_{b i m v}\right)$, single-vehicle crashes $\left(N_{b i s v}\right)$, vehicle-pedestrian crashes $\left(N_{\text {pedi }}\right)$, and vehicle-bicycle crashes $\left(N_{b i k e i}\right)$. The base conditions for calibrating the SPFs for multiple- and single-vehicle crashes at intersections are as follows:

- no left-turn lanes on intersection approaches,

- no right-turn lanes on intersection approaches,

- no lighting,

- permissive left-turn signal phasing for signalized intersections,

- no prohibition of RTOR maneuver for signalized intersections, and

- no red light cameras for signalized intersections.

In addition, the base conditions for applying the SPFs for vehicle-pedestrian crashes at signalized intersections include:

- no bus stops within $1,000 \mathrm{ft}$ from the center of the intersection,

- no schools within 1,000 ft from the center of the intersection, and

- no alcohol sales establishments within $1,000 \mathrm{ft}$ from the center of the intersection. 
First, $N_{\text {bimv }}$ and $N_{\text {bisv }}$ were estimated using Equations (4-16) and (4-17), respectively, as shown below:

$$
\begin{aligned}
& N_{\text {bimv }}=\exp \left[\gamma_{0}+\gamma_{1} \times \ln \left(A A D T_{\text {maj }}\right)+\gamma_{2} \times \ln \left(A A D T_{\text {min }}\right)\right] \\
& N_{\text {bisv }}=\exp \left[\delta_{0}+\delta_{1} \times \ln \left(A A D T_{\text {maj }}\right)+\delta_{2} \times \ln \left(A A D T_{\text {min }}\right)\right]
\end{aligned}
$$

where,

$A A D T_{m a j}=$ annual average daily traffic volume for major road (vehicles per day),

$A A D T_{\min }=$ annual average daily traffic volume for minor road (vehicles per day),

$\gamma_{0}, \gamma_{1}, \gamma_{2}=$ coefficients of SPF for multiple-vehicle crashes (values are given in Table B-7 in Appendix B), and

$\delta_{0}, \delta_{1}, \delta_{2}=$ coefficients of SPF for single-vehicle crashes (values are given in Table B-8 in Appendix B).

Next, the sum of $N_{b i m v}$ and $N_{\text {bisv }}$ was calculated to obtain $N_{\text {spfint }}$, which was then multiplied with CMFs to account for the effects of prevailing (i.e., non-base) conditions on predicted crash frequency at intersections. The CMFs $\left(C M F_{1 i}\right.$ through $\left.C M F_{6 i}\right)$ are discussed below:

\section{$C M F_{1 i}-$ Intersection Left-Turn Lanes}

The base condition for this CMF is the absence of left-turn lanes on the intersection approaches (i.e., $\left.C M F_{1 i}=1.00\right)$. The effect of presence of left-turn lanes on crash predictions was determined as:

- For three-leg stop-controlled intersections: 


$$
C M F_{1 i}=0.67^{\left(n_{L T-s t o p}\right)}
$$

- For four-leg signalized intersections:

$$
C M F_{1 i}=0.90^{\left(n_{L T-s i g n a l}\right)}
$$

where $n_{L T-s t o p}$ is the number of major-road approaches with left-turn lanes at stopcontrolled intersections and $n_{L T \text {-signal }}$ is the number of total approaches with leftturn lanes at signalized intersections.

\section{$C M F_{2 i}$ - Intersection Left-Turn Signal Phasing}

This CMF is applicable only to signalized intersections. There are three types of left-turn signal phasing at intersections: permissive only, protected only, and protected/permissive or permissive/protected. The base condition for this CMF is permissive only left-turn signal phasing (i.e., $C M F_{2 i}=1.00$ ). The effect of other left-turn signal phases on crash predictions was determined as follows:

- For protected/permissive or permissive/protected left-turn phasing:

$$
C M F_{2 i}=0.99^{\left(n_{p p}\right)}
$$

- For protected only left-turn phasing:

$$
C M F_{2 i}=0.94^{\left(n_{p r}\right)}
$$

where $n_{p p}$ is the number of approaches with protected/permissive or permissive/protected left-turn signal phasing and $n_{p r}$ is the number of approaches with protected only left-turn phasing. 


\section{$C M F_{3 i}-$ Intersection Right-Turn Lanes}

The base condition for this CMF is the absence of right-turn lanes on the intersection approaches (i.e., $C M F_{3 i}=1.00$ ). The effect of presence of right-turn lanes on intersection crashes was determined as:

- For three-leg stop-controlled intersections:

$$
C M F_{3 i}=0.86^{\left(n_{R T-s t o p}\right)}
$$

- For four-leg signalized intersections:

$$
C M F_{3 i}=0.96^{\left(n_{R T-\text { signal }}\right)}
$$

where $n_{R T \text {-stop }}$ is the number of major-road approaches with right-turn lanes at stop-controlled major street $n_{R T \text {-signal }}$ is the total number of approaches with rightturn lanes at signalized intersections.

\section{$C M F_{4 i}-$ Right Turn on Red (RTOR)}

This CMF is applicable only to signalized intersections. The base condition is permitting a RTOR at all approaches of a signalized intersection (i.e., $C M F_{4 i}=1.00$ ). The effect of prohibiting RTOR on crash predictions was determined as:

$$
C M F_{4 i}=0.98^{\left(n_{\text {prohib }}\right)}
$$

where $n_{\text {prohib }}$ is the number of approaches with prohibiting RTOR operation at signalized intersections.

$C M F_{5 i}-$ Lighting

The base condition for this CMF is the absence of lighting at an intersection (i.e., $\left.C M F_{5 i}=1.00\right)$. The effect of lighting on crash predictions was determined as follows: 


$$
C M F_{5 i}=1.0-0.38 \times p_{n i}
$$

where $p_{n i}$ is the proportion of total crashes at unlighted intersections that occur at night.

\section{$C M F_{6 i}-$ Red Light Camera}

The base condition for this $\mathrm{CMF}$ is the absence of red light cameras (i.e., $C M F_{6 i}=$ 1.00). The effect of presence of red light camera on crash predictions was determined as follows:

$$
C M F_{6 i}=1.0-0.26 \times p_{r a}+0.18 \times p_{r e}
$$

where $p_{r a}$ is the proportion of crashes that are multiple-vehicle, right-angle crashes and $p_{r e}$ is the proportion of crashes that are multiple-vehicle, rear-end crashes.

After determining $N_{b i}$, which is a product of $N_{s p f \text { int }}$ and CMFs (i.e., $C M F_{1 i}$ through $\left.C M F_{6 i}\right), N_{p e d i}$ was estimated using the following equations:

- For signalized intersections:

$$
N_{\text {pedi }}=N_{\text {pedbase }} \times C M F_{1 p} \times C M F_{2 p} \times C M F_{3 p}
$$

with

$$
\begin{array}{r}
N_{\text {pedbase }}=\exp \left[a+b \times \ln \left(A A D T_{\text {tot }}\right)+c \times \ln \left(\frac{A A D T_{\text {min }}}{A A D T_{\text {maj }}}\right)\right. \\
\left.+d \times \ln (\text { PedVol })+e \times n_{\text {laness }}\right]
\end{array}
$$

- For stop-controlled intersections:

$$
N_{\text {pedi }}=N_{b i} \times f_{\text {pedi }}
$$

where,

$$
\begin{aligned}
N_{\text {pedbase }} \quad & \text { predicted number of vehicle-pedestrian crashes per year } \\
& \text { for base conditions at signalized intersections, }
\end{aligned}
$$


$A A D T_{\text {tot }} \quad=$ sum of annual average daily traffic volumes for the major and minor roads (vehicles per day),

PedVol= sum of daily pedestrian volumes crossing all intersection legs (pedestrians per day),

$n_{\text {lanesx }}=$ maximum number of traffic lanes crossed by a pedestrian in any crossing maneuver at the intersection considering the presence of refuge islands,

$a, b, c, d, e=$ coefficients of SPF for vehicle-pedestrian crashes at signalized intersections (values are given in Table B-9 in Appendix B), $C M F_{1 p}, \ldots, C M F_{3 p}=$ crash modification factors for vehicle-pedestrian crashes at signalized intersections, and

$f_{\text {pedi }} \quad=$ pedestrian crash adjustment factor calculated as the ratio of total crashes (excluding vehicle-pedestrian and vehiclebicycle crashes) to vehicle-pedestrian crashes at stopcontrolled intersections.

Three CMFs (i.e., $C M F_{1 p}, C M F_{2 p}$, and $C M F_{3 p}$ ) were applied to account for the effects of bus stops, schools, and alcohol sales establishments on vehicle-pedestrian crashes at signalized intersections, as described below:

$C M F_{1 p}-$ Bus stops

The base condition for bus stops is the absence of bus stops within 1,000 $\mathrm{ft}$ of the intersection (i.e., $C M F_{1 p}=1.00$ ). The effect of presence of bus stops in the vicinity of the 
signalized intersection was determined as follows:

$$
C M F_{1 p}= \begin{cases}2.78, & \text { for } 1 \text { or } 2 \text { bus stops } \\ 4.75, & \text { for } 3 \text { or more bus stops }\end{cases}
$$

$C M F_{2 p}-$ Schools

The base condition for schools is the absence of a school within $1,000 \mathrm{ft}$ of the intersection (i.e., $C M F_{2 p}=1.00$ ). A value of 1.35 was used for $C M F_{2 p}$ to account for the effect of presence of school in the vicinity of the signalized intersection. $C M F_{3 p}-$ Alcohol sales establishments

The base condition for alcohol sales establishments is their absence within 1,000 $\mathrm{ft}$ of the intersection (i.e., $C M F_{3 p}=1.00$ ). The effect of alcohol sales establishments present in the vicinity of the signalized intersection was determined as follows:

$$
C M F_{3 p}= \begin{cases}1.12, & \text { for } 1 \text { to } 8 \text { alcohol sales establishments } \\ 1.56, & \text { for } 9 \text { or more alcohol sales establishments }\end{cases}
$$

In addition, $N_{\text {bikei }}$ was estimated using Equation (4-32) that applies to both stopcontrolled and signalized intersections, as shown below:

$$
N_{b i k e i}=N_{b i} \times f_{b i k e i}
$$

where $f_{\text {bikei }}$ is bicycle crash adjustment factor calculated as the ratio of total crashes (excluding vehicle-pedestrian and vehicle-bicycle crashes) to vehicle-bicycle crashes at intersections.

\subsection{Sensitivity Analysis of Calibration Sample Size}

The methodology used to determine minimum sample size for calibration of the HSM-default SPFs was based on the procedure used in the studies conducted by Banihashemi (2012) and Alluri et al. (2014) for sample size determination. In particular, 
a sensitivity analysis was performed to determine the effect of various sample sizes on calibration factors. For each site type, different sample sizes were selected. For each sample size, a number of iterations were taken to obtain a set of calibration factors. An acceptable limit was set so as to determine the degree of deviation of the calibration factors from its ideal value for each sample size. Based on the results of the sensitivity analysis, recommendations were provided on minimum sample size for estimating reliable calibration factor. The steps involved in the sensitivity analysis are described below:

Step 1 - Determine ideal calibration factor

For each roadway segment and intersection type, calibration factors were calculated using the entire data set. These calibration factors are regarded as ideal calibration factors. These ideal factors were developed based on the HSM procedures presented in the previous section.

Step 2 - Extract random samples

For all the segment types, samples were randomly extracted by taking the number of segments as follows: $(50+50 \times n)$, where $n$ takes the values from 0 to 9 . This indicates that a sample size consisting of 50 roadway segments was initially chosen and the size was increased by adding 50 segments in each subsequent sample. For two-lane undivided roads, four-lane divided arterials, and five-lane arterials with a TWLTL, the maximum sample size for the sensitivity measure consisted of 500 segments. However, three-lane arterials with a TWLTL and four-lane undivided arterials had an inventory of less than 500 segments. In this case, the sensitivity was measured with the maximum sample size of 300 segments for three-lane arterials with a TWLTL and 200 segments for four-lane 
undivided arterials. For intersections, random samples were extracted by taking number of intersections as follows: $(50+10 \times n)$, where $n$ takes the values from 0 to 10 . Similar to the segments, the initial sample size for intersections was 50; however, the size was increased by adding 10 intersections randomly in each successive sample. The maximum sample size comprised of 150 intersections for all the intersection types.

Step 3 - Determine calibration factors using random samples

For each specific sample size, 100 sets of data were randomly selected through simple random sampling process. This means, for example, when the sample size is 50 , each of the 100 data sets comprised 50 sites and, at each iteration, the sites were selected randomly from the entire data set. Calibration factors were then calculated for each of these 100 sets of data.

The mean and standard deviation of these calibration factors were used in the sensitivity analysis based on the assumption that the set of 100 calibration factors for each sample size followed a normal distribution.

Step 4 - Determine the Z-statistic

Following the assumption that the calibration factors are normally distributed, the probability of obtaining a calibration factor within acceptable limits (i.e., within $10 \%$ of ideal calibration factors) was determined. Therefore, the value that is equal to 0.90 times or $90 \%$ of the ideal calibration factor defines the minimum acceptable limit, and the value that is equal to 1.10 times or $110 \%$ of the ideal calibration factor is the maximum acceptable limit. For each sample size, these two values were transformed to the standardized values of the standard normal distribution curve (i.e., normal distribution curve with mean 0 and standard deviation 1), as given below: 


$$
\begin{aligned}
& Z_{\text {min }}=\frac{X_{\text {min }}-\mu}{\sigma} \\
& Z_{\text {max }}=\frac{X_{\text {max }}-\mu}{\sigma}
\end{aligned}
$$

where,

$$
\begin{aligned}
Z_{\text {min }}= & \text { value on the standard normal curve associated with } X_{\min }, \\
Z_{\max }= & \text { value on the standard normal curve associated with } X_{\max }, \\
X_{\min }= & \text { minimum acceptable limit of estimated calibration factor, } \\
X_{\max }= & \text { maximum acceptable limit of estimated calibration factor, } \\
\mu \quad & \text { mean of the normally distributed calibration factors for each sample size, } \\
& \text { and } \\
\sigma \quad & \text { standard deviation of the normally distributed calibration factors for each } \\
& \text { sample size. }
\end{aligned}
$$

\section{Step 5 - Determine the probability}

Two probabilities were calculated based on the $Z_{\min }$ and $Z_{\max }$ values under the standard normal curve area; one is the probability that the calibration factor is less than $90 \%$ of (or, 0.90 times) ideal calibration factor (i.e., $P\left(Z<Z_{\text {min }}\right)$, where, $Z$ is the value on the standard normal curve) and the other is the probability that the calibration factor is less than $110 \%$ of (or, 1.10 times) ideal calibration factor (i.e., $P\left(Z<Z_{\max }\right)$ ). The difference between the two probabilities (i.e., $\left.P\left(Z<Z_{\max }\right)-P\left(Z<Z_{\min }\right)\right)$ is the probability that the calibration factor would lie within $10 \%$ of the ideal calibration factor.

\section{Step 6-Recommend minimum sample size}

For each site type, the effect of sample size on calibration factor was studied by means of probability values computed in the previous step. A higher probability indicates 
a higher chance of obtaining a reliable calibration factor that would have less deviation from the ideal calibration factor. For example, a $95 \%$ probability value for a given sample size indicates that a data set with this particular size would produce a calibration factor with a high $95 \%$ chance that it would be within $10 \%$ of the ideal calibration factor. Based on high probability values, a minimum sample size is recommended for each segment and intersection type.

\subsection{Variable Importance}

This research applied the following three methods to rank the variables based on their relative effect on crash predictions:

- Negative Binomial (NB)

- Random Forests (RF)

- Boosted Regression Trees (BRT)

The detailed procedure of each of these methods is presented in the following subsections.

\subsubsection{Negative Binomial (NB)}

NB regression is the most widely used technique in modeling crash frequency data. The HSM-default SPFs were also developed using the NB regression method. The NB model is a member of the generalized linear models (GLMs), where a non-linear link function (e.g., logarithm) is used to establish the relationship between predictor variables and response variable (McCullagh and Nelder, 1989). The NB model formulates the relationship between expected crash frequency and various factors using the following equation: 


$$
\mu_{i}=\exp \left(\beta X_{i}+\varepsilon_{i}\right)
$$

where,

$$
\begin{aligned}
& \mu_{i}=\text { expected crash frequency at site } i \\
& X_{i}=\text { set of predictor variables for site } i \\
& \beta=\text { parameter or coefficient to be estimated, and } \\
& \varepsilon_{i}=\text { error term. }
\end{aligned}
$$

The probability distribution of the NB model is then shown as (Vogt and Bared, 1998; Hilbe, 2011):

$$
\mathrm{P}\left(Y_{i}=y_{i}\right)=\frac{\Gamma\left(y_{i}+(1 / k)\right)}{\Gamma\left(y_{i}+1\right) \Gamma(1 / k)}\left(\frac{k \mu_{i}}{1+k \mu_{i}}\right)^{y_{i}}\left(\frac{1}{1+k \mu_{i}}\right)^{1 / k}
$$

where,

$$
\begin{aligned}
& y_{i}=\text { number of crashes at site } i \text { over a time period, } \\
& k \quad=\text { overdispersion parameter of the NB model, and } \\
& \Gamma(.)=\text { a gamma function. }
\end{aligned}
$$

The likelihood function to estimate the NB model is shown below (Hilbe, 2011):

$$
L\left(\lambda_{i}\right)=\prod_{i} \frac{\Gamma\left(y_{i}+(1 / k)\right)}{\Gamma\left(y_{i}+1\right) \Gamma(1 / k)}\left(\frac{k \mu_{i}}{1+k \mu_{i}}\right)^{\mathrm{y}_{\mathrm{i}}}\left(\frac{1}{1+k \mu_{i}}\right)^{1 / k}
$$

This structure gives the variance of the NB model as follows (Vogt and Bared, 1998):

$$
V\left(y_{i}\right)=\mu_{i}\left(1+k \mu_{i}\right)
$$

\section{Variable Importance}

In order to investigate the relative effect of each explanatory variable using the NB regression technique, a number of NB models was developed. First, an intercept-only model was developed, which included only the constant term with no predictor variable. 
Then, separate models were developed with one predictor variable (including the intercept) in each model. The models are referred to as single-variable models. The

dispersion-based goodness-of-fit measure $\left(R_{k}^{2}\right)$ proposed by Miaou et al. (1996), which incorporates the effect of over-dispersion in crash frequency data, was determined for each of the models (i.e., intercept-only model and single-variable models). The value of $R_{k}^{2}$ was determined as follows:

$$
R_{k}^{2}=1-\left(k / \kappa_{\max }\right)
$$

where $\kappa_{\max }$ is the overdispersion parameter of the intercept-only model, and $\kappa$ is the overdispersion parameter of the single-variable model.

The higher the value of $R_{k}^{2}$, the better is the model fit. Since the models have only one predictor, the order of the $R_{k}^{2}$ values indicates the effect of that particular variable on overall crash predictions (Montella et al., 2008). The variables were, therefore, ranked by decreasing value of $R_{k}^{2}$.

\subsubsection{Random Forests $(\mathrm{RF})$}

The RF technique (Breiman, 2001) is developed based on two concepts: bagging (Breiman, 1996) and random feature subspace (Ho, 1995). The term "bagging" refers to the formation of trees (i.e., models) using a bootstrapping sample of the data set. In bagging, trees are grown independent of one another; successive trees do not carry information from the previously grown trees. The term "random feature subspace" refers to the predictor space formed by a subset of the randomly selected variables. At each node, the best split is determined from randomly selecting a few (subset) predictor 
variables rather than selecting all the variables. Note that a different set of predictor variables is selected for every node to examine over for the optimal split.

Algorithm

The algorithm of building a RF model is given below (Breiman, 2001; Liaw and Wiener, 2002; Blackwell, 2012):

1. Create bootstrap samples from the given data set. Bootstrapping means randomly selecting $n$ observations from the data set with replacement.

2. Fit a tree to the bootstrap sample. At each node, select a random subset of predictor variables $\left(m_{\text {try }}\right)$. Search over the subset $m_{\text {try }}$ to find the variable that produces maximum homogeneity at that node. Follow the procedure for the resulting nodes until a minimum specified criterion is reached.

3. Repeat Steps 1 and 2 for $n_{\text {tree }}$ trees.

4. Aggregate the results of the $n_{\text {tree }}$ trees and average out for predictions.

\section{Variable Importance}

The variable importance in an RF model is measured by increase in node purity values. The node purity of a variable corresponds to the summation of improvements due to splits by the variable in a tree. The cumulative improvements over all the trees are then averaged to give an overall increase in node purity or decrease in node impurity. The improvements are measured by mean decrease in residual sum of squares.

\subsubsection{Boosted Regression Trees (BRT)}

The BRT method is based on two powerful procedures: regression trees and boosting (Elith et al., 2008). Regression trees are decision-tree based models where the type of response variable is continuous. The term "boosting" refers to an ensemble 
approach that fits a number of trees in a sequential process. The basic idea is to give more emphasis to poorly fitted observations (i.e., the observations that highly deviate from the mean) based on the results from the previous tree alone rather than from all the other previously fitted trees (Bühlmann and Horton, 2007). The boosting procedure combines predictions from many weak models to produce a strong prediction and improve model accuracy (De'ath, 2007; Elith et al., 2008; Hastie et al., 2009). Therefore, unlike the RF method where trees are grown independently and no information is carried between trees, the BRT method builds trees sequentially by empowering the poor predictions from previous trees.

Algorithm

In BRT, once the first tree is fitted to the training data, the residuals based on predicted output from the tree are calculated. The observations with high residual values indicate poor fit and, therefore, are assigned more weight to fit by the next tree. In the next and subsequent steps, trees are fitted to the residuals of the previous tree, and so on. The BRT procedure is thus a forward and stage-wise procedure where "the existing trees are left unchanged; only the residual for each observation is re-estimated to reflect the contribution of the newly added tree" (Elith et al., 2008). The algorithm for the BRT model can be described as follows (De'ath, 2007; Hastie et al., 2009):

Let $x$ be a set of predictor variables and a function $f(x)$ be an approximation of the response variable $y$. The BRT model estimates the function as an additive expansion of basis functions, $b\left(x ; \gamma_{m}\right)$, as follows:

$$
f(x)=\sum_{m} f_{m}(x)=\sum_{m} \beta_{m} b\left(x ; \gamma_{m}\right)
$$


where $\beta_{m}(m=1,2, \ldots, M)$ are the expansion coefficients and $b\left(x ; \gamma_{m}\right)$ are single regression trees with the parameter $\gamma_{m}$ representing the split variables, their values at the splitting nodes, and the predicted values at the terminal nodes. The $\beta_{m}$ coefficients represent weights given to the nodes of each tree and determine how predictions from each of the trees are combined (De'ath, 2007). The $\beta_{m}$ and $\gamma_{m}$ parameters are estimated by minimizing a specified loss function, $L(y, f(x))$, which indicates a measure of prediction performance (e.g., deviance). Friedman (2001) formulated a numerical optimization technique called 'functional gradient descent' that approximates the solution of loss function minimization by the method of steepest descent for a forward stage-wise BRT model. The procedure can be summarized in the following steps (De'ath, 2007):

1. Initialize $f_{0}(x)$

2. For $m=1$ to $M$ (number of trees)

a) For $i=1$ to $N$ (number of observations), calculate the residuals,

$$
r_{i m}=-\left[\frac{\partial L\left(y_{i}, f\left(x_{i}\right)\right)}{\partial f\left(x_{i}\right)}\right]_{f(x)=f_{m-1}(x)}
$$

b) Fit a regression tree to $r_{i m}$ to estimate $\gamma_{m}$ of $b\left(x ; \gamma_{m}\right)$

c) Obtain the estimate $\beta_{m}$ by minimizing $L\left(y, f_{m-l}\left(x_{i}\right)+\beta b\left(x ; \gamma_{m}\right)\right)$

d) Update $f_{m}(x)=f_{m-1}\left(x_{i}\right)+\beta_{m} b\left(x ; \gamma_{m}\right)$

3. Calculate $f(x)=\sum_{m} f_{m}(x)$

Freidman (2002) also purported to bring in some randomness to the gradient boosting procedure in order to improve prediction performance and to reduce overfitting and computation time. This is attained by extracting a portion of the training data (usually $50 \%-75 \%$ ) without replacement at each iteration. 


\section{Regularization Parameters}

The sequential tree building process continues to add trees until all the observations are perfectly fit, which leads to overfitting of training data (Elith et al., 2008). Therefore, regularization of the BRT parameters is essential to fit a balanced model that reduces overfitting and improves prediction accuracy (Hastie et al., 2009). Regularization process usually involves optimizing three parameters including shrinkage, tree complexity, and number of trees to obtain a balance between bias and variance. Shrinkage, also called learning rate, is used to reduce the contribution of each tree in the model. It is introduced in Step 2(d) of the above described algorithm, as follows:

$$
f_{m}(x)=f_{m-1}\left(x_{i}\right)+\nu \beta_{m} b\left(x ; \gamma_{m}\right)
$$

where $v$ is the shrinkage parameter. A smaller shrinkage value (from 0.1 to as low as 0.0001 ) is typically applied to slower the learning process and better minimize the loss function; however, it requires more trees to be added to the model. For example, a 10fold reduction in shrinkage requires approximately 10 times more trees (De'ath, 2007) to be fitted in the boosting procedure. Tree complexity represents the depth of a tree implying interaction among predictor variables. A tree complexity of 1 corresponds to the main effect of predictor variables, and generates each tree with only two terminal nodes (having no internal nodes) called single decision stumps (Hastie et al., 2009). A tree complexity of 2 develops models with up to two-way interactions between variables (i.e., a maximum of two splits in each branch), and so on (Hastie et al., 2009). Elith et al. (2008) recommended fitting models with at least 1,000 trees. The success of a BRT model depends on the optimal settings of these regularization parameters.

\section{Variable Importance}


The variable importance in BRT is measured by the relative influence of predictor variables on the outcome. The influence of a predictor variable in a single tree is estimated by the number of times the variable is used to split the nodes and the squared improvement attributed to the tree due to the splits by the variable. The influence of the variable is summed over the ensemble of trees and the average value of the summation is regarded as the measure of variable importance in a BRT model (Friedman and Meulman, 2003). The sum of variable importance scores over all the variables is equal to 1 or $100 \%$. The variables are ranked with higher values of relative influence. The higher the variable importance score, the greater is the contribution of the variable on crash predictions.

\subsection{Summary}

In a nutshell, the following techniques were applied to accomplish the three objectives of this dissertation research:

- sensitivity analysis to evaluate the sample size criteria for determining calibration factor and to determine a minimum sample size required for segments and intersections;

- paired sample t-tests to validate the recommended intervals for updating the calibration factor; and

- negative binomial regression, random forests, and boosted regression trees methods to determine the influence of variables on crash predictions and to identify and prioritize the calibration variables. 


\section{CHAPTER 5}

\section{EVALUATION OF CALIBRATION CRITERIA}

This chapter presents the analytical results of evaluating the sample size criteria recommended in the HSM for the calibration process. It specifically discusses the effect of different sample sizes on the reliability of calibration factors. It further provides recommendations of the minimum sample size for calibrating the HSM-default SPFs for segment and intersection types on urban and suburban arterials in Florida. It then presents the results of a series of paired sample t-tests performed to determine how frequently agencies need to update calibration factors. Finally, the chapter summarizes the results related to the minimum sample size and calibration factor update frequency.

\subsection{Determination of Calibration Factors}

To determine the minimum sample size to estimate reliable calibration factors and to validate the recommendations on intervals for updating calibration factors, calibration factors were computed for each specific segment and intersection type(s) on urban and suburban arterials using the data set described in Chapter 3. The HSM-recommended process presented in Section 4.2 was used to derive calibration factors. Several steps in the calibration procedure called for using crash proportions either (a) provided in the HSM, or (b) derived from crash statistics specific to study locations. In this research, Florida-specific crash distributions were used where data were available; and in other instances, default proportions provided in the HSM were used. The following Floridaspecific crash proportions were used for deriving calibration factors: 
- proportion of total crashes that occurred at night on unlighted roadway segments $\left(p_{n r}\right)$

- proportion of total nighttime crashes that involved a fatality or injury on unlighted roadway segments $\left(p_{i n r}\right)$;

- proportion of total nighttime crashes that involved PDO on unlighted roadway segments $\left(p_{p n r}\right)$;

- pedestrian crash adjustment factors for intersections $\left(f_{\text {pedi }}\right)$;

- bicycle crash adjustment factors for intersections $\left(f_{b i k e i}\right)$;

- proportion of total crashes (excluding vehicle-pedestrian and vehicle-bicycle crashes) that occurred at night on unlighted intersections $\left(p_{n i}\right)$;

- proportion of total crashes (excluding vehicle-pedestrian and vehicle-bicycle crashes) that were multiple-vehicle, right-angle collisions on intersections $\left(p_{r a}\right)$; and

- proportion of total crashes (excluding vehicle-pedestrian and vehicle-bicycle crashes) that were multiple-vehicle, rear-end collisions on intersections ( $\left.p_{r e}\right)$.

Tables C-1 through C-7 in Appendix C provide the Florida-specific crash proportions. Based on the procedures in the HSM, two sets of calibration factors were calculated. The first set comprises calibration factors computed for each study year using that particular year of data (e.g., 2005, 2006, etc.). The second set comprises calibration factors computed using three consecutive years of data (e.g., data from 2005 to 2007, 2006 to 2008, etc.) The first set thus has eight calibration factors for each site type and the second set has six calibration factors for each site type, as shown in Tables 5-1 and 52 , respectively. 
Note that calibration factors using three years of data could be calculated using two approaches. One is to take the average of the yearly calibration factors and another is to divide the total number of observed crashes over three years by the total number of predicted crashes for the same period. The calibration factors obtained from these two approaches were almost identical (varied by as little as $0.1 \%$ in some cases). The calibration factors presented in Table 5-2 were calculated using the second approach.

TABLE 5-1 Calibration Factors Computed Using Single Year of Data for Urban and Suburban Arterials

\begin{tabular}{|c|c|c|c|c|c|c|c|c|}
\hline \multirow{2}{*}{ Site Type } & \multicolumn{8}{|c|}{ Calibration Factors } \\
\hline & 2005 & 2006 & 2007 & 2008 & 2009 & 2010 & 2011 & 2012 \\
\hline \multicolumn{9}{|c|}{ Segments } \\
\hline Two-lane undivided & 0.732 & 0.661 & 0.704 & 0.598 & 0.657 & 0.629 & 0.561 & 0.585 \\
\hline Three-lane with a TWLTL & 0.425 & 0.473 & 0.421 & 0.446 & 0.397 & 0.477 & 0.451 & 0.434 \\
\hline Four-lane undivided & 0.257 & 0.332 & 0.339 & 0.308 & 0.320 & 0.386 & 0.335 & 0.335 \\
\hline Four-lane divided & 0.741 & 0.702 & 0.710 & 0.673 & 0.664 & 0.680 & 0.583 & 0.673 \\
\hline Five-lane with a TWLTL & 0.366 & 0.352 & 0.358 & 0.350 & 0.376 & 0.431 & 0.366 & 0.394 \\
\hline \multicolumn{9}{|c|}{ Intersections } \\
\hline Three-leg stop-controlled & 0.863 & 0.817 & 0.797 & 0.821 & 0.733 & 0.837 & 0.830 & 1.017 \\
\hline Four-leg signalized & 2.574 & 2.433 & 2.412 & 2.429 & 2.516 & 2.760 & 2.440 & 2.799 \\
\hline
\end{tabular}

TABLE 5-2 Calibration Factors Computed Using Three Years of Data for Urban and Suburban Arterials

\begin{tabular}{|l|c|c|c|c|c|c|c|}
\hline \multirow{2}{*}{ Site Type } & \multicolumn{7}{|c|}{ Calibration Factors } \\
\cline { 2 - 8 } & 2005-2007 & 2006-2008 & 2007-2009 & 2008-2010 & 2009-2011 & 2010-2012 \\
\hline \multicolumn{7}{|c|}{ Segments } \\
\hline Two-lane undivided & 0.699 & 0.654 & 0.653 & 0.628 & 0.615 & 0.591 \\
\hline Three-lane with a TWLTL & 0.433 & 0.447 & 0.439 & 0.422 & 0.441 & 0.454 \\
\hline Four-lane undivided & 0.309 & 0.326 & 0.323 & 0.338 & 0.347 & 0.352 \\
\hline Four-lane divided & 0.718 & 0.695 & 0.682 & 0.672 & 0.642 & 0.645 \\
\hline Five-lane with a TWLTL & 0.358 & 0.353 & 0.361 & 0.385 & 0.391 & 0.397 \\
\hline \multicolumn{7}{|c|}{ Intersections } \\
\hline Three-leg stop-controlled & 0.826 & 0.812 & 0.784 & 0.796 & 0.798 & 0.891 \\
\hline Four-leg signalized & 2.472 & 2.425 & 2.452 & 2.565 & 2.572 & 2.666 \\
\hline
\end{tabular}




\subsection{Effect of Sample Size on Calibration Factors}

The HSM states that "for each facility type, the desirable minimum sample size for the calibration dataset is 30 to 50 sites" (AASHTO, 2010). Also, the selected calibration data set should represent a minimum of 100 crashes per year. A sensitivity analysis was performed to evaluate the minimum sample size criteria. The effect of sample size on calibration factors was measured by taking samples of different sizes and determining a normal probability that the calibration factors estimated using a sample size would be within $10 \%$ of calibration factors computed using the entire data set. The higher the probability, the greater is the reliability to determine calibration factors for a given sample size.

Different samples between 50 and 500 sites for segments and between 50 and 150 sites for intersections were randomly selected for analysis. For each sample size, 100 subsets each with equal number of sites were randomly generated and calibration factors were determined for each subset of data. Thus, a set of 100 calibration factors was estimated for each sample size. Calibration factors in each set were assumed to have a normal distribution. Based on the assumption of normal probability distribution, the mean and standard deviation of each set of calibration factors were used to determine the probability that the estimated calibration factor lies within $10 \%$ of the actual calibration factor calculated from the entire data set. Finally, the effect of sample size on calibration factor estimations was evaluated based on this probability.

Similar to Tables 5-1 and 5-2, two sets of calibration factors (i.e., one using single year of data and the other using three years of data) were calculated for each sample size. 
The purpose was to investigate whether or not any distinctive features are prevalent between these two sets related to sample size and calibration factors.

\subsubsection{Segments}

Tables 5-3 and 5-4 show the sensitivity analysis results for all the five types of urban and suburban arterial roadway segments. Table 5-3 provides the probability values when the sensitivity was measured by estimating calibration factors using individual years of data, and Table 5-4 provides the probability values when the sensitivity was measured by computing calibration factors using three consecutive years of data. Note that ideal calibration factor refers to calibration factor estimated using entire data set for either individual years or three consecutive years.

The results from Tables 5-3 and 5-4 show that for a given sample size, the probability that the calibration factor would be within $10 \%$ of ideal calibration factor is higher when calibration factors were estimated using three years of data compared to that when calibration factors were estimated using single year of data. For example, in case of two-lane undivided arterials, when calibration factors were estimated using single year of data, a sample size of 450 sites yielded probabilities varying between $86 \%$ and $91 \%$, whereas a reduced sample size of 350 sites resulted in probabilities between $88 \%$ and 91\% when calibration factors were estimated using three years of data. Similarly, for four-lane divided arterials, Table 5-3 shows that a sample size of 450 sites resulted in probabilities between $81 \%$ and $89 \%$, while Table 5-4 shows that a reduced sample size of 300 sites generated probabilities between $89 \%$ and $94 \%$. Similar results were also observed for other roadway types. This indicates that sample size would be smaller when multiple years of data are used to determine reliable calibration factors. 
The minimum sample size required to estimate reliable calibration factor was also found to vary by roadway type. An increased sample size is usually required for the facility that has large population of roadway miles. Two-lane undivided roads have an inventory of 616.7 miles of data and require more than 120 miles of roadway data ( $20 \%$ of entire roadway length) to produce a reliable calibration factor (i.e., there is an $80 \%$ probability that the estimated calibration factor is within $10 \%$ of ideal calibration factor). On the other hand, three-lane arterials with a TWLTL and four-lane undivided arterials have only 67.0 and 52.2 miles of roadway, respectively, and require less than 50 miles of roadway data to produce calibration factors with a high probability of being within $10 \%$ of the ideal calibration factor. Four-lane divided arterials have the largest inventory of roadway miles; however, only 85 miles of roadway data yielded reliable calibration factors for this site type. It shows that although four-lane divided arterials have larger inventory of roadway miles compared to two-lane undivided roads, they require a smaller sample size to generate a reliable calibration factor. A higher average annual crash frequency of 4.30 crashes per mile per year for four-lane divided highways might be attributed to the outcome of the smaller sample size requirement. This indicates that crash frequency also has an impact on the minimum required sample size. 
TABLE 5-3 Sensitivity Analysis Results for Urban and Suburban Arterial Segments Using Individual Years of Data

\begin{tabular}{|c|c|c|c|c|c|c|c|c|c|c|c|c|}
\hline \multirow{2}{*}{ Site Type } & \multirow{2}{*}{$\begin{array}{l}\text { Sample } \\
\text { Size }\end{array}$} & \multirow{2}{*}{$\begin{array}{c}\text { Average } \\
\text { Roadway } \\
\text { Length } \\
\text { (mi) }\end{array}$} & \multirow{2}{*}{$\begin{array}{c}\text { Percent } \\
\text { of Entire } \\
\text { Length } \\
(\%) \\
\end{array}$} & \multicolumn{9}{|c|}{$\begin{array}{l}\text { Probability that Estimated Calibration Factor Is } \\
\text { Within } 10 \% \text { of Ideal Calibration Factor (\%) }\end{array}$} \\
\hline & & & & 2005 & 2006 & 2007 & 2008 & 2009 & 2010 & 2011 & 2012 & Average \\
\hline \multirow{10}{*}{$\begin{array}{l}\text { Two-Lane } \\
\text { Undivided }\end{array}$} & 50 & 17.6 & 2.9 & 34 & 34 & 35 & 33 & 34 & 37 & 32 & 33 & 34 \\
\hline & 100 & 34.5 & 5.6 & 41 & 46 & 52 & 42 & 47 & 45 & 44 & 42 & 45 \\
\hline & 150 & 51.8 & 8.4 & 54 & 60 & 56 & 58 & 58 & 55 & 61 & 52 & 57 \\
\hline & 200 & 68.5 & 11.1 & 68 & 66 & 68 & 61 & 62 & 65 & 62 & 61 & 64 \\
\hline & 250 & 85.4 & 13.8 & 66 & 76 & 74 & 76 & 68 & 67 & 76 & 62 & 71 \\
\hline & 300 & 102.3 & 16.6 & 75 & 73 & 71 & 73 & 75 & 76 & 74 & 72 & 74 \\
\hline & 350 & 119.8 & 19.4 & 84 & 81 & 77 & 84 & 81 & 73 & 79 & 79 & 80 \\
\hline & 400 & 137.2 & 22.3 & 87 & 87 & 91 & 79 & 86 & 84 & 86 & 80 & 85 \\
\hline & 450 & 154.8 & 25.1 & 89 & 86 & 86 & 86 & 91 & 89 & 89 & 86 & 88 \\
\hline & 500 & 172.2 & 27.9 & 94 & 93 & 95 & 95 & 85 & 85 & 88 & 88 & 90 \\
\hline \multirow{6}{*}{$\begin{array}{c}\text { Three-Lane } \\
\text { with a } \\
\text { TWLTL }\end{array}$} & 50 & 9.5 & 14.2 & 29 & 33 & 31 & 32 & 28 & 33 & 37 & 43 & 33 \\
\hline & 100 & 18.9 & 28.2 & 46 & 53 & 45 & 49 & 50 & 52 & 56 & 56 & 51 \\
\hline & 150 & 27.9 & 41.6 & 63 & 62 & 57 & 63 & 67 & 69 & 69 & 65 & 64 \\
\hline & 200 & 37.5 & 55.9 & 74 & 77 & 75 & 77 & 79 & 80 & 82 & 82 & 78 \\
\hline & 250 & 46.5 & 69.3 & 91 & 90 & 89 & 91 & 86 & 92 & 92 & 94 & 91 \\
\hline & 300 & 56.1 & 83.7 & 98 & 97 & 99 & 97 & 99 & 99 & 99 & 99 & 98 \\
\hline \multirow{4}{*}{$\begin{array}{l}\text { Four-Lane } \\
\text { Undivided }\end{array}$} & 50 & 9.8 & 18.8 & 45 & 43 & 34 & 44 & 38 & 36 & 39 & 38 & 40 \\
\hline & 100 & 19.4 & 37.2 & 63 & 70 & 61 & 66 & 59 & 59 & 57 & 60 & 62 \\
\hline & 150 & 29.3 & 56.2 & 81 & 87 & 79 & 84 & 78 & 81 & 82 & 82 & 82 \\
\hline & 200 & 39.3 & 75.3 & 95 & 97 & 94 & 95 & 95 & 93 & 95 & 92 & 95 \\
\hline \multirow{10}{*}{$\begin{array}{l}\text { Four-Lane } \\
\text { Divided }\end{array}$} & 50 & 14.3 & 1.0 & 45 & 37 & 41 & 41 & 39 & 35 & 39 & 33 & 39 \\
\hline & 100 & 28.9 & 2.1 & 54 & 52 & 56 & 59 & 59 & 54 & 52 & 51 & 55 \\
\hline & 150 & 42.2 & 3.0 & 61 & 61 & 69 & 67 & 59 & 60 & 64 & 59 & 63 \\
\hline & 200 & 57.8 & 4.1 & 72 & 73 & 80 & 78 & 76 & 71 & 73 & 70 & 74 \\
\hline & 250 & 71.2 & 5.1 & 80 & 75 & 78 & 79 & 76 & 71 & 72 & 75 & 76 \\
\hline & 300 & 84.7 & 6.1 & 81 & 86 & 89 & 86 & 79 & 82 & 81 & 77 & 83 \\
\hline & 350 & 99.8 & 7.1 & 86 & 84 & 88 & 87 & 84 & 81 & 83 & 83 & 85 \\
\hline & 400 & 112.2 & 8.0 & 86 & 84 & 90 & 87 & 84 & 85 & 85 & 85 & 86 \\
\hline & 450 & 127.8 & 9.1 & 87 & 87 & 87 & 89 & 87 & 86 & 81 & 89 & 87 \\
\hline & 500 & 141.3 & 10.1 & 94 & 93 & 91 & 95 & 91 & 89 & 88 & 94 & 92 \\
\hline \multirow{10}{*}{$\begin{array}{c}\text { Five-Lane } \\
\text { with a } \\
\text { TWLTL }\end{array}$} & 50 & 12.7 & 4.7 & 45 & 53 & 47 & 46 & 46 & 45 & 43 & 47 & 47 \\
\hline & 100 & 24.5 & 9.1 & 64 & 66 & 64 & 66 & 57 & 57 & 64 & 57 & 62 \\
\hline & 150 & 37.1 & 13.8 & 69 & 77 & 72 & 75 & 67 & 64 & 69 & 59 & 69 \\
\hline & 200 & 49.3 & 18.3 & 79 & 84 & 81 & 75 & 71 & 74 & 79 & 71 & 77 \\
\hline & 250 & 62.0 & 23.0 & 88 & 92 & 86 & 87 & 87 & 87 & 82 & 76 & 86 \\
\hline & 300 & 74.9 & 27.8 & 90 & 95 & 93 & 90 & 92 & 89 & 95 & 82 & 91 \\
\hline & 350 & 87.0 & 32.3 & 95 & 95 & 95 & 93 & 88 & 90 & 92 & 82 & 91 \\
\hline & 400 & 99.0 & 36.8 & 95 & 96 & 98 & 93 & 95 & 92 & 93 & 87 & 94 \\
\hline & 450 & 111.8 & 41.6 & 98 & 97 & 97 & 99 & 98 & 94 & 98 & 95 & 97 \\
\hline & 500 & 124.1 & 46.1 & 99 & 100 & 99 & 99 & 99 & 97 & 98 & 95 & 98 \\
\hline
\end{tabular}


TABLE 5-4 Sensitivity Analysis Results for Urban and Suburban Arterial Segments Using Three Consecutive Years of Data

\begin{tabular}{|c|c|c|c|c|c|c|c|c|c|c|}
\hline \multirow{2}{*}{ Site Type } & \multirow{2}{*}{$\begin{array}{c}\text { Sample } \\
\text { Size }\end{array}$} & \multirow{2}{*}{$\begin{array}{c}\text { Average } \\
\text { Roadway } \\
\text { Length } \\
\text { (mi) }\end{array}$} & \multirow{2}{*}{\begin{tabular}{|c|} 
Percent \\
of Entire \\
Length \\
$(\%)$ \\
\end{tabular}} & \multicolumn{7}{|c|}{$\begin{array}{c}\text { Probability that Estimated Calibration Factor Is } \\
\text { Within 10\% of Ideal Calibration Factor (\%) }\end{array}$} \\
\hline & & & & $\begin{array}{c}2005- \\
2007\end{array}$ & $\begin{array}{l}2006- \\
2008\end{array}$ & $\begin{array}{l}2007- \\
2009\end{array}$ & $\begin{array}{l}2008- \\
2010\end{array}$ & $\begin{array}{c}2009- \\
2011\end{array}$ & $\begin{array}{l}2010- \\
2012\end{array}$ & Average \\
\hline \multirow{10}{*}{$\begin{array}{l}\text { Two-Lane } \\
\text { Undivided }\end{array}$} & 50 & 17.6 & 2.9 & 44 & 43 & 45 & 46 & 44 & 44 & 44 \\
\hline & 100 & 34.5 & 5.6 & 57 & 59 & 60 & 58 & 57 & 57 & 58 \\
\hline & 150 & 51.8 & 8.4 & 68 & 69 & 69 & 72 & 73 & 74 & 71 \\
\hline & 200 & 68.5 & 11.1 & 80 & 77 & 76 & 76 & 76 & 77 & 77 \\
\hline & 250 & 85.4 & 13.8 & 84 & 87 & 84 & 82 & 80 & 81 & 83 \\
\hline & 300 & 102.3 & 16.6 & 80 & 82 & 84 & 85 & 88 & 90 & 85 \\
\hline & 350 & 119.8 & 19.4 & 89 & 90 & 91 & 90 & 88 & 90 & 90 \\
\hline & 400 & 137.2 & 22.3 & 95 & 95 & 96 & 94 & 95 & 95 & 95 \\
\hline & 450 & 154.8 & 25.1 & 94 & 94 & 96 & 98 & 98 & 98 & 96 \\
\hline & 500 & 172.2 & 27.9 & 100 & 99 & 100 & 98 & 94 & 96 & 98 \\
\hline \multirow{6}{*}{$\begin{array}{c}\text { Three-Lane } \\
\text { with a } \\
\text { TWLTL }\end{array}$} & 50 & 9.5 & 14.2 & 38 & 35 & 38 & 40 & 43 & 47 & 40 \\
\hline & 100 & 18.9 & 28.2 & 49 & 55 & 56 & 60 & 62 & 67 & 58 \\
\hline & 150 & 27.9 & 41.6 & 70 & 71 & 71 & 80 & 83 & 83 & 76 \\
\hline & 200 & 37.5 & 55.9 & 85 & 86 & 85 & 88 & 89 & 90 & 87 \\
\hline & 250 & 46.5 & 69.3 & 95 & 95 & 94 & 96 & 96 & 98 & 96 \\
\hline & 300 & 56.1 & 83.7 & 100 & 99 & 100 & 100 & 100 & 100 & 100 \\
\hline \multirow{4}{*}{$\begin{array}{l}\text { Four-Lane } \\
\text { Undivided }\end{array}$} & 50 & 9.8 & 18.8 & 48 & 48 & 49 & 52 & 48 & 46 & 49 \\
\hline & 100 & 19.4 & 37.2 & 80 & 79 & 76 & 77 & 72 & 71 & 76 \\
\hline & 150 & 29.3 & 56.2 & 93 & 91 & 91 & 93 & 94 & 94 & 93 \\
\hline & 200 & 39.3 & 75.3 & 99 & 99 & 99 & 99 & 99 & 98 & 99 \\
\hline \multirow{10}{*}{$\begin{array}{l}\text { Four-Lane } \\
\text { Divided }\end{array}$} & 50 & 14.3 & 1.0 & 50 & 47 & 49 & 46 & 47 & 44 & 47 \\
\hline & 100 & 28.9 & 2.1 & 64 & 66 & 69 & 69 & 66 & 64 & 66 \\
\hline & 150 & 42.2 & 3.0 & 74 & 77 & 75 & 72 & 72 & 72 & 74 \\
\hline & 200 & 57.8 & 4.1 & 87 & 88 & 89 & 86 & 86 & 83 & 87 \\
\hline & 250 & 71.2 & 5.1 & 86 & 86 & 86 & 84 & 82 & 82 & 84 \\
\hline & 300 & 84.7 & 6.1 & 94 & 96 & 93 & 92 & 90 & 89 & 92 \\
\hline & 350 & 99.8 & 7.1 & 93 & 94 & 94 & 92 & 90 & 90 & 92 \\
\hline & 400 & 112.2 & 8.0 & 93 & 93 & 93 & 92 & 91 & 92 & 92 \\
\hline & 450 & 127.8 & 9.1 & 93 & 95 & 94 & 94 & 93 & 94 & 94 \\
\hline & 500 & 141.3 & 10.1 & 96 & 98 & 97 & 96 & 97 & 96 & 97 \\
\hline \multirow{10}{*}{$\begin{array}{c}\text { Five-Lane } \\
\text { with a } \\
\text { TWLTL }\end{array}$} & 50 & 12.7 & 4.7 & 59 & 59 & 55 & 52 & 52 & 56 & 56 \\
\hline & 100 & 24.5 & 9.1 & 78 & 77 & 77 & 74 & 71 & 73 & 75 \\
\hline & 150 & 37.1 & 13.8 & 86 & 87 & 82 & 77 & 75 & 74 & 80 \\
\hline & 200 & 49.3 & 18.3 & 91 & 89 & 84 & 81 & 82 & 85 & 85 \\
\hline & 250 & 62.0 & 23.0 & 96 & 96 & 95 & 94 & 92 & 90 & 94 \\
\hline & 300 & 74.9 & 27.8 & 98 & 98 & 97 & 96 & 96 & 95 & 97 \\
\hline & 350 & 87.0 & 32.3 & 99 & 99 & 98 & 95 & 95 & 94 & 97 \\
\hline & 400 & 99.0 & 36.8 & 99 & 99 & 99 & 97 & 97 & 97 & 98 \\
\hline & 450 & 111.8 & 41.6 & 100 & 100 & 100 & 99 & 99 & 99 & 100 \\
\hline & 500 & 124.1 & 46.1 & 100 & 100 & 100 & 100 & 100 & 100 & 100 \\
\hline
\end{tabular}




\subsubsection{Intersections}

Tables 5-5 and 5-6 show the sensitivity analysis results for urban and suburban intersection types when calibration factors were calculated using data from individual year and three consecutive years, respectively.

Similar to segments, on urban three-leg stop-controlled intersections, the probability that the estimated calibration factor would lie within $10 \%$ of ideal calibration factor is higher when calibration factors were estimated using three years of data rather than using one year of data. In the case of four-leg signalized intersections, the difference in probabilities for the same sample size between the two sets of calibration factors is between $3 \%$ and $4 \%$. An extremely high annual frequency of 17.16 crashes per intersection might be the reason for obtaining such low differences in probability values. This indicates that the number of years of data used for estimating calibration factors has less impact for the site types that experience high frequency of observed crashes. Furthermore, because of low observed crash frequency at three-leg stop-controlled intersections, the minimum required sample size considered about $50 \%$ of the total intersections. On the contrary, less than $20 \%$ of the total number of four-leg signalized intersections yielded calibration factors with greater than $80 \%$ probability to be within $10 \%$ of the ideal calibration factor.

\subsection{Recommendations on Minimum Sample Size}

The recommendations on minimum sample size for deriving reliable calibration factors are based on the results from Tables 5-3 and 5-4 for segments and the results from Tables 5-5 and 5-6 for intersections. The recommendations for each segment and intersection type are provided in the following subsections. 
TABLE 5-5 Sensitivity Analysis Results for Urban and Suburban Intersections Using Individual Years of Data

\begin{tabular}{|c|c|c|c|c|c|c|c|c|c|c|c|}
\hline \multirow{2}{*}{ Site Type } & \multirow{2}{*}{$\begin{array}{c}\text { Sample } \\
\text { Size }\end{array}$} & \multirow{2}{*}{$\begin{array}{c}\text { Percent of } \\
\text { Intersections } \\
(\%)\end{array}$} & \multicolumn{9}{|c|}{$\begin{array}{c}\text { Probability that Estimated Calibration Factor Is Within } \\
10 \% \text { of Ideal Calibration Factor (\%) }\end{array}$} \\
\hline & & & 2005 & 2006 & 2007 & 2008 & 2009 & 2010 & 2011 & 2012 & Average \\
\hline \multirow{11}{*}{$\begin{array}{l}\text { Three-leg } \\
\text { stop- } \\
\text { controlled }\end{array}$} & 50 & 16 & 46 & 48 & 43 & 46 & 42 & 51 & 42 & 44 & 45 \\
\hline & 60 & 19 & 51 & 49 & 50 & 53 & 46 & 55 & 45 & 50 & 50 \\
\hline & 70 & 22 & 55 & 59 & 58 & 59 & 51 & 56 & 51 & 57 & 56 \\
\hline & 80 & 25 & 61 & 65 & 66 & 67 & 57 & 64 & 57 & 64 & 63 \\
\hline & 90 & 28 & 63 & 67 & 66 & 70 & 65 & 66 & 60 & 68 & 66 \\
\hline & 100 & 32 & 66 & 69 & 68 & 74 & 66 & 72 & 64 & 74 & 69 \\
\hline & 110 & 35 & 71 & 71 & 72 & 78 & 67 & 75 & 64 & 77 & 72 \\
\hline & 120 & 38 & 74 & 76 & 74 & 77 & 71 & 77 & 70 & 80 & 75 \\
\hline & 130 & 41 & 80 & 78 & 78 & 80 & 74 & 82 & 74 & 84 & 79 \\
\hline & 140 & 44 & 82 & 80 & 78 & 81 & 77 & 84 & 75 & 87 & 81 \\
\hline & 150 & 47 & 82 & 83 & 83 & 85 & 82 & 85 & 77 & 89 & 83 \\
\hline \multirow{11}{*}{$\begin{array}{l}\text { Four-leg } \\
\text { signalized }\end{array}$} & 50 & 13 & 71 & 72 & 71 & 72 & 71 & 69 & 63 & 67 & 70 \\
\hline & 60 & 15 & 78 & 79 & 77 & 79 & 76 & 74 & 68 & 70 & 75 \\
\hline & 70 & 17 & 85 & 85 & 82 & 85 & 80 & 80 & 73 & 77 & 81 \\
\hline & 80 & 20 & 88 & 88 & 85 & 88 & 85 & 84 & 74 & 76 & 84 \\
\hline & 90 & 22 & 92 & 92 & 90 & 91 & 87 & 87 & 75 & 77 & 86 \\
\hline & 100 & 25 & 93 & 93 & 93 & 92 & 89 & 89 & 80 & 79 & 89 \\
\hline & 110 & 27 & 94 & 94 & 95 & 94 & 91 & 92 & 82 & 83 & 91 \\
\hline & 120 & 30 & 96 & 95 & 97 & 96 & 95 & 94 & 86 & 87 & 93 \\
\hline & 130 & 32 & 97 & 98 & 98 & 98 & 97 & 96 & 89 & 90 & 95 \\
\hline & 140 & 35 & 98 & 99 & 99 & 99 & 98 & 97 & 92 & 93 & 97 \\
\hline & 150 & 37 & 99 & 99 & 99 & 99 & 99 & 98 & 94 & 94 & 98 \\
\hline
\end{tabular}


TABLE 5-6 Sensitivity Analysis Results for Urban and Suburban Intersections Using Three Consecutive Years of Data

\begin{tabular}{|c|c|c|c|c|c|c|c|c|c|}
\hline \multirow{2}{*}{ Site Type } & \multirow{2}{*}{$\begin{array}{c}\text { Sample } \\
\text { Size }\end{array}$} & \multirow{2}{*}{$\begin{array}{c}\text { Percent of } \\
\text { Intersections } \\
(\%)\end{array}$} & \multicolumn{7}{|c|}{$\begin{array}{l}\text { Probability that Estimated Calibration Factor Is Within } \\
10 \% \text { of Ideal Calibration Factor }(\%)\end{array}$} \\
\hline & & & $\begin{array}{l}2005- \\
2007\end{array}$ & $\begin{array}{c}2006- \\
2008\end{array}$ & $\begin{array}{c}2007- \\
2009\end{array}$ & $\begin{array}{c}2008- \\
2010\end{array}$ & $\begin{array}{c}2009- \\
2011\end{array}$ & $\begin{array}{c}2010- \\
2012\end{array}$ & Average \\
\hline \multirow{11}{*}{$\begin{array}{l}\text { Three-leg } \\
\text { stop- } \\
\text { controlled }\end{array}$} & 50 & 16 & 52 & 52 & 50 & 55 & 54 & 54 & 53 \\
\hline & 60 & 19 & 60 & 61 & 57 & 59 & 58 & 60 & 59 \\
\hline & 70 & 22 & 65 & 67 & 64 & 63 & 61 & 65 & 64 \\
\hline & 80 & 25 & 71 & 75 & 72 & 71 & 69 & 73 & 72 \\
\hline & 90 & 28 & 73 & 77 & 76 & 77 & 76 & 77 & 76 \\
\hline & 100 & 32 & 75 & 79 & 78 & 79 & 79 & 81 & 79 \\
\hline & 110 & 35 & 79 & 83 & 81 & 82 & 80 & 84 & 82 \\
\hline & 120 & 38 & 82 & 85 & 82 & 83 & 84 & 86 & 84 \\
\hline & 130 & 41 & 85 & 86 & 85 & 86 & 87 & 90 & 87 \\
\hline & 140 & 44 & 86 & 87 & 86 & 88 & 88 & 90 & 88 \\
\hline & 150 & 47 & 91 & 91 & 89 & 90 & 89 & 92 & 90 \\
\hline \multirow{11}{*}{$\begin{array}{l}\text { Four-leg } \\
\text { signalized }\end{array}$} & 50 & 13 & 75 & 76 & 76 & 74 & 73 & 71 & 74 \\
\hline & 60 & 15 & 81 & 82 & 82 & 80 & 77 & 75 & 80 \\
\hline & 70 & 17 & 87 & 88 & 87 & 85 & 82 & 81 & 85 \\
\hline & 80 & 20 & 90 & 91 & 90 & 88 & 85 & 82 & 88 \\
\hline & 90 & 22 & 94 & 94 & 93 & 91 & 86 & 83 & 90 \\
\hline & 100 & 25 & 95 & 95 & 94 & 92 & 89 & 86 & 92 \\
\hline & 110 & 27 & 96 & 97 & 96 & 94 & 92 & 89 & 94 \\
\hline & 120 & 30 & 98 & 98 & 98 & 96 & 94 & 92 & 96 \\
\hline & 130 & 32 & 99 & 99 & 99 & 98 & 96 & 94 & 98 \\
\hline & 140 & 35 & 99 & 99 & 99 & 99 & 97 & 96 & 98 \\
\hline & 150 & 37 & 100 & 100 & 100 & 99 & 98 & 97 & 99 \\
\hline
\end{tabular}

\subsubsection{Segments}

On urban and suburban two-lane undivided arterials, when calibration factors were calculated using individual years of data, samples consisting of 350 sites showed an $80 \%$ overall probability that the estimated calibration factor would lie within $10 \%$ of the ideal calibration factor. On the other hand, when calibration factors were calculated using three consecutive years of data, the probability increased to $90 \%$ with the sample size of 350 sites. Therefore, a minimum sample size of 350 sites is recommended for calibrating the HSM-default SPFs for urban and suburban two-lane undivided arterials in Florida. 
This corresponds to a road network of approximately 120 miles with an average crash frequency of 230 crashes per year.

On urban and suburban three-lane arterials with a center TWLTL, the calibration factor estimated with a sample size consisting of 200 sites was found to lie within $10 \%$ of the ideal calibration factor at an overall $78 \%$ probability when one year of data were used and at an $87 \%$ probability when three consecutive years of data were used. A minimum sample size of 200 segments is, therefore, recommended for calibrating the HSM-default SPFs for urban and suburban three-lane arterial sections with a center TWLTL in Florida. This sample size corresponds to approximately 40 miles of roadway with an average of 125 crashes per year.

For urban and suburban four-lane undivided arterial sections, a sample of 150 sites produced calibration factors within $10 \%$ of the ideal calibration factor at an overall $82 \%$ probability using individual years of data and at an overall $93 \%$ probability using three consecutive years of data. Therefore, a minimum sample size of 150 segments consisting of approximately 30 miles of roadway with an average of 135 crashes per year is recommended for urban and suburban four-lane undivided arterials. Note that four-lane undivided arterial sections have the smallest inventory in urban and suburban area in Florida and, therefore, the required sample size with fewer number of segments actually covers more than $50 \%$ of total roadway miles.

Urban and suburban four-lane divided arterials constitute the largest inventory of roadway data in urban Florida. Table 5-3 shows that when calibration factors were developed using individual years of data, a sample size consisting of 300 sites resulted in an $83 \%$ probability that the estimated calibration factor would lie within $10 \%$ of the ideal 
calibration factor. On the other hand, Table 5-4 shows that when calibration factors were estimated using three consecutive years of data, the same sample of 300 sites showed a high $92 \%$ probability that the estimated calibration factor would lie within $10 \%$ of the ideal calibration factor. Also, increasing the sample size from 300 to 450 segments hardly improved the probability that the calibration factors would lie within $10 \%$ of the ideal calibration factor. Therefore, 300 sites might be adequate to produce a reliable calibration factor for urban and suburban four-lane divided arterials. A sample of urban four-lane divided roads consisting of 300 sites covers approximately 85 miles of roadway and experiences an average frequency of 360 crashes per year in Florida.

On urban and suburban five-lane arterial sections with a center TWLTL, samples consisting of 250 segments resulted in an $86 \%$ probability on average when calibration factors were estimated using individual years of data and in a $94 \%$ probability when calibration factors were estimated using three consecutive years of data. It is obvious that a sample size of 250 sites could be able to produce a high-quality calibration factor that would lie within $10 \%$ of the ideal calibration factor. This sample size represents approximately 60 miles of urban and suburban five-lane arterial roadways with an average of 360 crashes per year in Florida.

\subsubsection{Intersections}

As can be seen in Tables 5-5 and 5-6, on urban and suburban three-leg stopcontrolled intersections with samples consisting of 110 to 150 intersections, the probability that the estimated calibration factor would lie within $10 \%$ of the ideal calibration factor improved only slightly. Therefore, a sample size that produced a probability of at least $80 \%$ might be adequate considering reliability and cost 
effectiveness. When three years of data were used, a sample size of 140 intersections resulted in an overall $88 \%$ probability that the estimated calibration factor would lie within $10 \%$ of the ideal calibration factor. On the other hand, when individual years of data were used, the sample of 140 intersections yielded an overall $81 \%$ probability that the estimated calibration factor would lie within $10 \%$ of the ideal calibration factor. Therefore, a sample size consisting of 140 urban and suburban three-leg stop-controlled intersections with an average of 250 annual crashes is recommended.

Four-leg signalized intersections are the most predominant intersection type in urban and suburban areas in Florida. Furthermore, these intersections experiences extremely high crash frequencies. Because of these prevailing crash statistics, a relatively small sample size might yield a reliable calibration factor. Tables 5-5 and 5-6 show that a sample size of 50 intersections could produce a calibration factor that would vary within $10 \%$ of the ideal calibration factor with a $70 \%$ probability using single year of data, and with a $74 \%$ probability using three years of data. However, the standard deviation of the calibration factor for a sample of 50 intersections was relatively high. It is found that a sample of 100 intersections might be adequate to have a reliable calibration factor with lower standard deviation. For a sample of 100 intersections, the probability that the estimated calibration factor lies within $10 \%$ of ideal calibration factor is $89 \%$ when calibration factors were estimated using individual years of data and $92 \%$ when calibration factors were estimated using three consecutive years of data. Therefore, a minimum sample size of 100 intersections with an average crash frequency of 1,700 annual crashes is recommended for urban and suburban four-leg signalized intersections in Florida. 


\subsection{Validation of the Recommended Frequency for Calibration Factor Updates}

According to the HSM, "new values of the calibration factors be derived at least every two to three years, and some HSM users may prefer to develop calibration factors on an annual basis" (AASHTO, 2010). In order to validate the recommendations on suggested intervals for estimating new calibration factors, the following three scenarios were considered:

- Scenario 1: whether calibration factors calculated every three years are different;

- Scenario 2: whether calibration factors calculated every two years are different; and

- Scenario 3: whether calibration factors calculated every year are different.

The scenarios were evaluated based on the calibration factors computed using three years of data. The calibration factors developed in the sensitivity analysis process were used in this regard. Thus, a total of six sets of calibration factors were available for evaluating the scenarios, as designated below:

- calibration factor computed using data from 2005 to $2007\left(C F_{1}\right)$

- calibration factor computed using data from 2006 to $2008\left(\mathrm{CF}_{2}\right)$

- calibration factor computed using data from 2007 to $2009\left(C_{3}\right)$

- calibration factor computed using data from 2008 to $2010\left(C F_{4}\right)$

- calibration factor computed using data from 2009 to $2011\left(C F_{5}\right)$

- calibration factor computed using data from 2010 to $2012\left(C F_{6}\right)$

Paired sample t-tests were conducted for each scenario with the following hypotheses:

Scenario 1: 
Null Hypothesis $\left(H_{o}\right)$ : There is no significant difference between the means of calibration factors estimated every three years

$$
\left(\mu_{C F_{1}}=\mu_{C F_{4}}, \mu_{C F_{2}}=\mu_{C F_{5}}, \mu_{C F_{3}}=\mu_{C F_{6}}\right)
$$

Alternative Hypothesis $\left(H_{a}\right)$ : There exists significant difference between any of the means of calibration factors estimated every three years

$$
\left(\mu_{C F_{1}} \neq \mu_{\mathrm{CF}_{4}}, \mu_{C F_{2}} \neq \mu_{C F_{5}} \text {, or } \mu_{C F_{3}} \neq \mu_{C F_{6}}\right)
$$

Scenario 2:

Null Hypothesis $\left(H_{o}\right)$ : There is no significant difference between the means of calibration factors estimated every two years

$$
\left(\mu_{C F_{1}}=\mu_{C F_{3}}, \mu_{C F_{2}}=\mu_{C F_{4}}, \mu_{C F_{3}}=\mu_{C F_{5}}, \mu_{C F_{4}}=\mu_{C F_{6}}\right)
$$

Alternative Hypothesis $\left(H_{a}\right)$ : There exists significant difference between any of the means of calibration factors estimated every two years

$$
\left(\mu_{C F_{1}} \neq \mu_{C F_{3}}, \mu_{C F_{2}} \neq \mu_{C F_{4}}, \mu_{C F_{3}} \neq \mu_{C F_{5}} \text {, or } \mu_{C F_{4}} \neq \mu_{C F_{6}}\right)
$$

Scenario 3:

Null Hypothesis $\left(H_{o}\right)$ : There is no significant difference between the means of calibration factors estimated every year

$$
\left(\mu_{C F_{1}}=\mu_{C F_{2}}, \mu_{C F_{2}}=\mu_{C F_{3}}, \mu_{C F_{3}}=\mu_{C F_{4}}, \mu_{C F_{4}}=\mu_{C F_{5}}, \mu_{C F_{5}}=\mu_{C F_{6}}\right)
$$

Alternative Hypothesis $\left(H_{a}\right)$ : There exists significant difference between any of the means of calibration factors estimated every year

$$
\left(\mu_{C F_{1}}=\mu_{C F_{2}}, \mu_{C F_{2}}=\mu_{C F_{3}}, \mu_{C F_{3}}=\mu_{C F_{4}}, \mu_{C F_{4}}=\mu_{C F_{5}}, \text { or } \mu_{C F_{5}}=\mu_{C F_{6}}\right)
$$

Tables 5-7 through 5-9 present the paired t-test results for urban and suburban arterial segments for Scenarios 1, 2, and 3, respectively, and Tables 5-10 through 5-12 
present the results for urban and suburban intersections for Scenarios 1, 2, and 3, respectively. The sample size in the tables refers to the recommended sample size for the site types determined in Section 5.3. For those specific sample sizes, the corresponding set of 100 calibration factors computed during the sensitivity analysis was used. The degree of freedom $(d o f)$ is determined by $(n-1)$, where $n$ is the number of calibration factors in each set. Therefore, the values of $n$ and $d o f$ are 100 and 99, respectively, in all the cases. The level of significance was chosen to be 0.05 . It means if $p$-value is less than 0.05 , the null hypothesis is to be rejected implying that there is statistically significant difference between means of calibration factors.

\subsubsection{Segments}

Table 5-7 shows the paired t-test results for Scenario 1 that compares the means

of calibration factors between every three years (i.e., between $\mu_{C F_{1}}$ and $\mu_{C F_{4}}, \mu_{C F_{2}}$ and $\mu_{C F_{5}}$, and $\mu_{C_{3}}$ and $\mu_{C F_{6}}$ ) for urban and suburban roadway segment types. A p-value of greater than 0.05 is observed for three-lane arterials with a TWLTL in two of the three instances indicating that there is no statistically significant difference in calibration factors. For other segment types, the p-values are consistently lower than 0.05 rejecting the null hypothesis that there is no difference in calibration factors if updated every three years.

Table 5-8 shows the paired t-test results for Scenario 2 that compares the means of calibration factors between every two years (i.e., between $\mu_{C F_{1}}$ and $\mu_{C_{3}}, \mu_{C_{2}}$ and $\mu_{C F_{4}}, \mu_{C F_{3}}$ and $\mu_{C F_{5}}$, and $\mu_{C F_{4}}$ and $\mu_{C F_{6}}$ ) for urban and suburban roadway segment types. 
A single instance of a p-value of greater than 0.05 is observed for five-lane arterials with

a TWLTL.

TABLE 5-7 Paired t-Test Results for Urban and Suburban Arterial Segments for Scenario 1

\begin{tabular}{|c|l|c|c|c|c|}
\hline Hypotheses & Segment Type & Sample Size & t-statistic & dof & p-value \\
\hline \multirow{4}{*}{$H_{o}: \mu_{C F_{1}}=\mu_{C F_{4}}$} & Two-lane undivided & 350 & 18.5522 & 99 & $<0.0001$ \\
\cline { 2 - 6 }$H_{a}: \mu_{C F_{1}} \neq \mu_{C F_{4}}$ & Three-lane with a TWLTL & 200 & 0.0870 & 99 & 0.9309 \\
\cline { 2 - 6 } & Four-lane undivided & 150 & 17.7493 & 99 & $<0.0001$ \\
\cline { 2 - 6 } & Four-lane divided & 300 & 13.1454 & 99 & $<0.0001$ \\
\hline \multirow{4}{*}{$H_{o}: \mu_{C F_{2}}=\mu_{C F_{5}}$} & Five-lane with a TWLTL & 250 & 13.8904 & 99 & $<0.0001$ \\
\cline { 2 - 6 }$H_{a}: \mu_{C F_{2}} \neq \mu_{C F_{5}}$ & Two-lane undivided & 350 & 11.2242 & 99 & $<0.0001$ \\
\cline { 2 - 6 } & Four-lane undivided & 200 & 2.2966 & 99 & 0.0238 \\
\cline { 2 - 6 } & Four-lane divided & 300 & 16.7807 & 99 & $<0.0001$ \\
\hline \multirow{3}{*}{$H_{o}: \mu_{C F_{3}}=\mu_{C F_{6}}$} & Five-lane with a TWLTL & 250 & 19.3848 & 99 & $<0.0001$ \\
\cline { 2 - 6 }$H_{a}: \mu_{C F_{3}} \neq \mu_{C F_{6}}$ & Two-lane undivided & 350 & 17.7592 & 99 & $<0.0001$ \\
\cline { 2 - 6 } & Four-lane undivided & 150 & 17.3338 & 99 & $<0.0001$ \\
\cline { 2 - 6 } & Four-lane divided & 300 & 9.7984 & 99 & $<0.0001$ \\
\cline { 2 - 6 } & Five-lane with a TWLTL & 250 & 19.6841 & 99 & $<0.0001$ \\
\hline
\end{tabular}

TABLE 5-8 Paired t-Test Results for Urban and Suburban Arterial Segments for Scenario 2

\begin{tabular}{|c|l|c|c|c|c|}
\hline Hypotheses & Segment Type & Sample Size & t-statistic & dof & p-value \\
\hline \multirow{4}{*}{$H_{o}: \mu_{C F_{1}}=\mu_{C F_{3}}$} & Two-lane undivided & 350 & 16.7954 & 99 & $<0.0001$ \\
\cline { 2 - 6 }$H_{a}: \mu_{C F_{1}} \neq \mu_{C F_{3}}$ & Three-lane with a TWLTL & 200 & 10.5452 & 99 & $<0.0001$ \\
\cline { 2 - 6 } & Four-lane undivided & 150 & 10.4203 & 99 & $<0.0001$ \\
\cline { 2 - 6 } & Four-lane divided & 300 & 12.2660 & 99 & $<0.0001$ \\
\cline { 2 - 6 } & Five-lane with a TWLTL & 250 & 1.1070 & 99 & 0.2710 \\
\hline \multirow{4}{*}{$H_{o}: \mu_{C F_{2}}=\mu_{C F_{4}}$} & Two-lane undivided & 350 & 8.3696 & 99 & $<0.0001$ \\
\cline { 2 - 6 }$H_{a}: \mu_{C F_{2}} \neq \mu_{C F_{4}}$ & Three-lane with a TWLTL & 200 & 3.3544 & 99 & 0.0011 \\
\cline { 2 - 6 } & Four-lane undivided & 150 & 6.2042 & 99 & $<0.0001$ \\
\cline { 2 - 6 } & Four-lane divided & 300 & 10.3368 & 99 & $<0.0001$ \\
\hline \multirow{4}{*}{$H_{o}: \mu_{C F_{3}}=\mu_{C F_{5}}$} & Five-lane with a TWLTL & 250 & 21.5045 & 99 & $<0.0001$ \\
\cline { 2 - 6 }$H_{a}: \mu_{C F_{3}} \neq \mu_{C F_{5}}$ & Two-lane undivided & 350 & 13.6029 & 99 & $<0.0001$ \\
\cline { 2 - 6 } & Four-lane undivided & 200 & 7.9114 & 99 & $<0.0001$ \\
\cline { 2 - 6 } & Four-lane divided & 300 & 15.6857 & 99 & $<0.0001$ \\
\hline \multirow{2}{*}{$H_{o}: \mu_{C F_{4}}=\mu_{C F_{6}}$} & Five-lane with a TWLTL & 250 & 20.3431 & 99 & $<0.0001$ \\
\cline { 2 - 6 }$H_{a}: \mu_{C F_{4}} \neq \mu_{C F_{6}}$ & Two-lane undivided & 350 & 13.3128 & 99 & $<0.0001$ \\
\cline { 2 - 6 } & Four-lane undivided & 150 & 11.7779 & 99 & $<0.0001$ \\
\hline
\end{tabular}




\begin{tabular}{|c|l|c|c|c|c|}
\hline Hypotheses & Segment Type & Sample Size & t-statistic & dof & p-value \\
\hline \multirow{2}{*}{} & Four-lane divided & 300 & 7.1753 & 99 & $<0.0001$ \\
\cline { 2 - 6 } & Five-lane with a TWLTL & 250 & 6.3586 & 99 & $<0.0001$ \\
\hline
\end{tabular}

Table 5-9 shows the paired t-test results for Scenario 3 which compares the means of calibration factors between two consecutive years (i.e., between $\mu_{C F_{1}}$ and $\mu_{C_{2}}, \mu_{C F_{2}}$ and $\mu_{C F_{3}}, \mu_{C F_{3}}$ and $\mu_{C F_{4}}, \mu_{C F_{4}}$ and $\mu_{C F_{5}}$, and $\mu_{C F_{5}}$ and $\mu_{C F_{6}}$ ) for urban and suburban roadway segment types. There are two instances in which p-values are greater than 0.05 , indicating that calibration factors were not significantly different. However, the null hypothesis was rejected for all the other instances with p-values less than 0.05 .

In summary, results from the paired t-tests for all three scenarios indicate that the calibration factors should be updated every year.

TABLE 5-9 Paired t-Test Results for Urban and Suburban Arterial Segments for Scenario 3

\begin{tabular}{|c|c|c|c|c|c|}
\hline Hypotheses & Segment Type & Sample Size & t-statistic & dof & p-value \\
\hline \multirow{5}{*}{$\begin{array}{l}H_{o}: \mu_{C F_{1}}=\mu_{C F_{2}} \\
H_{a}: \mu_{C F_{1}} \neq \mu_{C F_{2}}\end{array}$} & Two-lane undivided & 350 & 24.1479 & 99 & $<0.0001$ \\
\hline & Three-lane with a TWLTL & 200 & 5.6538 & 99 & $<0.0001$ \\
\hline & Four-lane undivided & 150 & 21.1443 & 99 & $<0.0001$ \\
\hline & Four-lane divided & 300 & 11.0638 & 99 & $<0.0001$ \\
\hline & Five-lane with a TWLTL & 250 & 7.5111 & 99 & $<0.0001$ \\
\hline \multirow{5}{*}{$\begin{array}{l}H_{o}: \mu_{C F_{2}}=\mu_{C F_{3}} \\
H_{a}: \mu_{C F_{2}} \neq \mu_{C F_{3}}\end{array}$} & Two-lane undivided & 350 & 0.8626 & 99 & 0.3904 \\
\hline & Three-lane with a TWLTL & 200 & 20.9502 & 99 & $<0.0001$ \\
\hline & Four-lane undivided & 150 & 5.6277 & 99 & $<0.0001$ \\
\hline & Four-lane divided & 300 & 8.0854 & 99 & $<0.0001$ \\
\hline & Five-lane with a TWLTL & 250 & 8.5945 & 99 & $<0.0001$ \\
\hline \multirow{5}{*}{$\begin{array}{l}H_{o}: \mu_{C F_{3}}=\mu_{C F_{4}} \\
H_{a}: \mu_{C F_{3}} \neq \mu_{C F_{4}}\end{array}$} & Two-lane undivided & 350 & 11.5880 & 99 & $<0.0001$ \\
\hline & Three-lane with a TWLTL & 200 & 12.0039 & 99 & $<0.0001$ \\
\hline & Four-lane undivided & 150 & 14.9484 & 99 & $<0.0001$ \\
\hline & Four-lane divided & 300 & 6.9709 & 99 & $<0.0001$ \\
\hline & Five-lane with a TWLTL & 250 & 25.6385 & 99 & $<0.0001$ \\
\hline \multirow{5}{*}{$\begin{array}{l}H_{o}: \mu_{C F_{4}}=\mu_{C F_{5}} \\
H_{a}: \mu_{C F_{4}} \neq \mu_{C F_{5}}\end{array}$} & Two-lane undivided & 350 & 8.3383 & 99 & $<0.0000$ \\
\hline & Three-lane with a TWLTL & 200 & 0.3104 & 99 & 0.7569 \\
\hline & Four-lane undivided & 150 & 10.5301 & 99 & $<0.0001$ \\
\hline & Four-lane divided & 300 & 15.0342 & 99 & $<0.0001$ \\
\hline & Five-lane with a TWLTL & 250 & 4.7879 & 99 & $<0.0001$ \\
\hline$H_{0}: \mu_{C E_{r}}=\mu_{C E_{r}}$ & Two-lane undivided & 350 & 12.4224 & 99 & $<0.0001$ \\
\hline
\end{tabular}




\begin{tabular}{|c|l|c|c|c|c|}
\hline Hypotheses & Segment Type & Sample Size & t-statistic & dof & p-value \\
\hline$H_{a}: \mu_{C F_{5}} \neq \mu_{C F_{6}}$ & Three-lane with a TWLTL & 200 & 11.2660 & 99 & $<0.0001$ \\
\cline { 2 - 6 } & Four-lane undivided & 150 & 6.2796 & 99 & $<0.0001$ \\
\cline { 2 - 6 } & Four-lane divided & 300 & 4.5539 & 99 & $<0.0001$ \\
\cline { 2 - 6 } & Five-lane with a TWLTL & 250 & 3.7286 & 99 & 0.0003 \\
\hline
\end{tabular}

\subsubsection{Intersections}

Tables 5-10 through 5-12 provide the paired t-test results for three-leg stopcontrolled and four-leg signalized intersections corresponding to Scenarios 1, 2, and 3, respectively. As can be observed from these tables, there is only one instance where the p-value is greater than 0.05 . This is observed when the mean of $C F_{4}$ (i.e., $\mu_{C F_{4}}$ ) was compared with that of $\mathrm{CF}_{5}$ (i.e., $\mu_{C_{5}}$ ) for three-leg stop-controlled intersections. There are no other instances where the null hypotheses cannot be rejected, i.e., there is significant difference in calibration factors between every three years, every two years, and every year. The results, therefore, indicate that it is reasonable to update calibration factors annually.

TABLE 5-10 Paired t-Test Results for Urban and Suburban Intersections for Scenario 1

\begin{tabular}{|c|l|c|c|c|c|}
\hline Hypotheses & Intersection Type & Sample Size & t-statistic & dof & p-value \\
\hline$H_{o}: \mu_{C F_{1}}=\mu_{C F_{4}}$ & Three-leg stop-controlled & 140 & 6.9376 & 99 & $<0.0001$ \\
\cline { 2 - 6 }$H_{a}: \mu_{C F_{1}} \neq \mu_{C F_{4}}$ & Four-leg signalized & 100 & 9.8072 & 99 & $<0.0001$ \\
\hline$H_{o}: \mu_{C F_{2}}=\mu_{C F_{5}}$ & Three-leg stop-controlled & 140 & 3.8793 & 99 & 0.0002 \\
\cline { 2 - 6 }$H_{a}: \mu_{C F_{2}} \neq \mu_{C F_{5}}$ & Four-leg signalized & 100 & 14.9669 & 99 & $<0.0001$ \\
\hline$H_{o}: \mu_{C F_{3}}=\mu_{C F_{6}}$ & Three-leg stop-controlled & 140 & 25.0998 & 99 & $<0.0001$ \\
\cline { 2 - 6 }$H_{a}: \mu_{C F_{3}} \neq \mu_{C F_{6}}$ & Four-leg signalized & 100 & 20.3219 & 99 & $<0.0001$ \\
\hline
\end{tabular}


TABLE 5-11 Paired t-Test Results for Urban and Suburban Intersections for Scenario 2

\begin{tabular}{|c|l|c|c|c|c|}
\hline Hypotheses & Intersection Type & Sample Size & t-statistic & dof & p-value \\
\hline$H_{o}: \mu_{C F_{1}}=\mu_{C F_{3}}$ & Three-leg stop-controlled & 140 & 13.0614 & 99 & $<0.0001$ \\
\cline { 2 - 6 }$H_{a}: \mu_{C F_{1}} \neq \mu_{C F_{3}}$ & Four-leg signalized & 100 & 3.5291 & 99 & 0.0006 \\
\hline$H_{o}: \mu_{C F_{2}}=\mu_{C F_{4}}$ & Three-leg stop-controlled & 140 & 4.9481 & 99 & $<0.0001$ \\
\cline { 2 - 6 }$H_{a}: \mu_{C F_{2}} \neq \mu_{C F_{4}}$ & Four-leg signalized & 100 & 18.3952 & 99 & $<0.0001$ \\
\hline$H_{o}: \mu_{C F_{3}}=\mu_{C F_{5}}$ & Three-leg stop-controlled & 140 & 4.2843 & 99 & $<0.0001$ \\
\cline { 2 - 7 }$H_{a}: \mu_{C F_{3}} \neq \mu_{C F_{5}}$ & Four-leg signalized & 100 & 15.7717 & 99 & $<0.0001$ \\
\hline$H_{o}: \mu_{C F_{4}}=\mu_{C F_{6}}$ & Three-leg stop-controlled & 140 & 24.7470 & 99 & $<0.0001$ \\
\cline { 2 - 7 }$H_{a}: \mu_{C F_{4}} \neq \mu_{C F_{6}}$ & Four-leg signalized & 100 & 12.6930 & 99 & $<0.0001$ \\
\hline
\end{tabular}

TABLE 5-12 Paired t-Test Results for Urban and Suburban Intersections for Scenario 3

\begin{tabular}{|c|l|c|c|c|c|}
\hline Hypotheses & Intersection Type & Sample Size & t-statistic & dof & p-value \\
\hline$H_{o}: \mu_{C F_{1}}=\mu_{C F_{2}}$ & Three-leg stop-controlled & 140 & 6.5028 & 99 & $<0.0001$ \\
\cline { 2 - 6 }$H_{a}: \mu_{C F_{1}} \neq \mu_{C F_{2}}$ & Four-leg signalized & 100 & 15.7696 & 99 & $<0.0001$ \\
\hline$H_{o}: \mu_{C F_{2}}=\mu_{C F_{3}}$ & Three-leg stop-controlled & 140 & 14.5076 & 99 & $<0.0001$ \\
\cline { 2 - 6 }$H_{a}: \mu_{C F_{2}} \neq \mu_{C F_{3}}$ & Four-leg signalized & 100 & 7.4210 & 99 & $<0.0001$ \\
\hline$H_{o}: \mu_{C F_{3}}=\mu_{C F_{4}}$ & Three-leg stop-controlled & 140 & 6.4931 & 99 & $<0.0001$ \\
\cline { 2 - 7 }$H_{a}: \mu_{C F_{3}} \neq \mu_{C F_{4}}$ & Four-leg signalized & 100 & 22.9308 & 99 & $<0.0001$ \\
\hline$H_{o}: \mu_{C F_{4}}=\mu_{C F_{5}}$ & Three-leg stop-controlled & 140 & 0.1994 & 99 & 0.8424 \\
\cline { 2 - 7 }$H_{a}: \mu_{C F_{4}} \neq \mu_{C F_{5}}$ & Four-leg signalized & 100 & 2.5648 & 99 & 0.0118 \\
\hline$H_{o}: \mu_{C F_{5}}=\mu_{C F_{6}}$ & Three-leg stop-controlled & 140 & 39.9057 & 99 & $<0.0001$ \\
\cline { 2 - 6 }$H_{a}: \mu_{C F_{5}} \neq \mu_{C F_{6}}$ & Four-leg signalized & 100 & 17.8326 & 99 & $<0.0001$ \\
\hline
\end{tabular}

\subsection{Summary}

The sensitivity analysis results show that the minimum sample size criteria specified in the HSM for calibration were not sufficient to produce reliable calibration factors. The calibration factor estimated from sample data was considered to be reliable if there is a high probability that the estimated calibration factor (i.e., calibration factor computed using a sample data set) would lie within $10 \%$ of the ideal calibration factor (i.e., calibration factor computed using the entire data set). 
Tables 5-13 and 5-14 give the required minimum sample sizes and the estimated probabilities to obtain reliable calibration factors for urban and suburban segment and intersection types, respectively. For each site type, the tables also give the corresponding percentage of total length for segments, percentage of total intersections for intersections, and the total number of crashes per year to yield a reliable calibration factor. The paired sample t-test results show that calibration factors need to be updated each year for all the urban and suburban arterial segment and intersection types.

TABLE 5-13 Recommended Sample Size for Urban and Suburban Arterial Segments

\begin{tabular}{|l|c|c|c|c|c|}
\hline Segment Type & $\begin{array}{c}\text { Number } \\
\text { of } \\
\text { Segments }\end{array}$ & $\begin{array}{c}\text { Roadway } \\
\text { Length } \\
\text { (miles) }\end{array}$ & $\begin{array}{c}\text { Percent } \\
\text { of Total } \\
\text { Length }\end{array}$ & $\begin{array}{c}\text { Number } \\
\text { of } \\
\text { Crashes } \\
\text { per Year }\end{array}$ & $\begin{array}{c}\text { P(Estimated CF } \\
\text { Is Within 10\% } \\
\text { of Ideal CF) } \\
\text { (\%) }\end{array}$ \\
\hline Two-lane undivided arterials & 350 & 120 & 19 & 230 & $80-90$ \\
\hline Three-lane arterials with a TWLTL & 200 & 40 & 56 & 125 & $78-87$ \\
\hline Four-lane undivided arterials & 150 & 30 & 56 & 135 & $82-93$ \\
\hline Four-lane divided arterials & 300 & 85 & 6 & 360 & $83-92$ \\
\hline Five-lane arterials with a TWLTL & 250 & 60 & 23 & 360 & $86-94$ \\
\hline
\end{tabular}

${ }^{*} \mathrm{CF}$ indicates calibration factor.

TABLE 5-14 Recommended Sample Size for Urban and Suburban Intersections

\begin{tabular}{|l|c|c|c|c|}
\hline Intersection Type & $\begin{array}{c}\text { Number } \\
\text { of } \\
\text { Intersections }\end{array}$ & $\begin{array}{c}\text { Percent of } \\
\text { Total } \\
\text { Intersections }\end{array}$ & $\begin{array}{c}\text { Number } \\
\text { of Crashes } \\
\text { per Year }\end{array}$ & $\begin{array}{c}\text { P(Estimated CF Is } \\
\text { Within 10\% of } \\
\text { Ideal CF) } \\
\text { (\%) }\end{array}$ \\
\hline Three-leg stop-controlled & 140 & 44 & 250 & $81-88$ \\
\hline Four-leg signalized & 100 & 25 & 1,700 & $89-92$ \\
\hline
\end{tabular}

${ }^{*} \mathrm{CF}$ indicates calibration factor. 


\section{CHAPTER 6}

\section{DATA PRIORITIZATION}

This chapter presents the variable prioritization results for urban and suburban arterial segments. To identify and prioritize the influential variables for calibrating the HSM predictive models, the following three methods were applied: negative binomial (NB) regression, random forests $(\mathrm{RF})$ and boosted regression trees (BRT). These three different methods are considered based on the assumption that each method might produce different results in determining the influence of each variable on crash predictions and is, therefore, likely to rank variables differently. The variables were ranked based on $R_{k}^{2}$ values using the NB method, by increase in node purity values using the RF method, and by percentage of relative influence using the BRT method. The results from the models developed using all the three methods were combined to identify and prioritize the influential variables for urban and suburban arterial segments. The first section of this chapter presents the variable specification for the analysis and the following three sections present the development of models and the outcome of variable importance measures using the $\mathrm{NB}, \mathrm{RF}$, and $\mathrm{BRT}$ methods, respectively. The prioritization of variables is presented thereafter.

\subsection{Variable Specification}

This research developed models to identify influential variables for urban and suburban arterial segments. Table 6-1 shows the name and type of the HSM variables for calibrating the default SPFs. Note that not all the HSM variables were considered in this research for developing models due to obvious correlation between the variables. For 
example, type of on-street parking, parking by land use type, and curb length with onstreet parking are only applicable for locations with on-street parking facility. It is clearly discernible that these variables would be highly correlated to the presence of on-street parking variable. Similarly, offset to roadside objects is only applicable for locations with roadside objects. To avoid the known effect of correlation, these variables (i.e., type of on-street parking, parking by land use type, curb length with on-street parking, and offset to roadside objects) were not considered in developing the models. Additionally, driveways were included in the models in terms of driveway density, i.e., driveways per mile.

TABLE 6-1 Variables in the HSM for Urban and Suburban Arterial Segments

\begin{tabular}{|c|c|}
\hline Variable & Type \\
\hline AADT & Continuous \\
\hline Segment length & Continuous \\
\hline Median width ${ }^{*}$ & $\begin{array}{l}\text { Categorical (10 levels: } 10^{\prime}, 20^{\prime}, 30^{\prime}, 40^{\prime}, 50^{\prime}, 60^{\prime}, 70^{\prime}, 80^{\prime} \text {, } \\
\left.90^{\prime}, 100^{\prime}\right)\end{array}$ \\
\hline Major commercial driveways & Continuous \\
\hline Major residential driveways & Continuous \\
\hline Major industrial driveways & Continuous \\
\hline Minor commercial driveways & Continuous \\
\hline Minor residential driveways & Continuous \\
\hline Minor industrial driveways & Continuous \\
\hline Other driveways & Continuous \\
\hline Roadside object density & Continuous \\
\hline Speed limit & Categorical (Two levels: $\leq 30 \mathrm{mph},>30 \mathrm{mph}$ ) \\
\hline Presence of on-street parking & Categorical (Two levels: absent, present) \\
\hline Presence of lighting & Categorical (Two levels: absent, present) \\
\hline Presence of automated enforcement & Categorical (Two levels: absent, present) \\
\hline Type of on-street parking & Categorical (Two levels: angle, parallel) \\
\hline Parking by land use type & $\begin{array}{l}\text { Categorical (Two levels: residential/other, commercial } \\
\text { or industrial/institutional) }\end{array}$ \\
\hline Curb length with on-street parking & Continuous \\
\hline Offset to roadside objects & Categorical (Seven levels: 2', 5', 10', 15', 20', 25', $\geq 30^{\prime}$ ) \\
\hline
\end{tabular}

* Median width is applicable only for four-lane divided arterials. 
The five most recent years (i.e., from 2008 to 2012) of crash data were used to develop the models using the three methods. Therefore, the dependent variable is the total number of crashes in five years. The logarithm of the product of segment length and number of years used in the analysis (i.e., five) is included as an offset factor in the model formulation to obtain the output (i.e., crash predictions) in crashes per mile per year.

\subsection{NB Regression Models}

Tables 6-2 through 6-6 show the goodness-of-fit measures of the single-variable NB models for two-lane undivided arterials, three-lane arterials with a TWLTL, four-lane undivided arterials, four-lane divided arterials, and five-lane arterials with a TWLTL, respectively. The models are referred by the corresponding variable in the tables. The overdispersion parameter of the intercept-only models is shown first and then the variables are ranked by increasing values of overdispersion parameter $(k)$ and decreasing values of $R_{k}^{2}$. The p-value of each variable is also provided to identify significant variables. A significance level of 0.05 was set to determine whether or not variables are significantly different from zero. As such, the variables with the p-values less than 0.05 are statistically significant at $95 \%$ confidence level. The statistical significance of categorical variables was measured by analysis of variance (ANOVA) test. The ANOVA test was conducted between the intercept-only model and the model that included the categorical variable. Note that the logarithm of AADT was used in the NB models to determine the effect of AADT. As can be observed from the tables, AADT is the most influential variable. However, the rank of other variables differs in each of the five models. It is observed from the tables that there are a total of seven significant variables in two-lane undivided arterials, four significant variables in three-lane arterials with a 
TWLTL, three significant variables in four-lane undivided arterials, nine significant variables in four-lane divided arterials, and six significant variables in five-lane arterials with a TWLTL. Besides AADT (specifically, natural logarithm of AADT), minor commercial driveway density is another variable that is significant in all the five segment types.

TABLE 6-2 NB Model Results for Urban and Suburban Two-Lane Undivided Arterials

\begin{tabular}{|l|c|c|c|}
\hline Variable & $\boldsymbol{k}$ & $\boldsymbol{R}_{\boldsymbol{k}}^{2}$ & p-value \\
\hline Intercept-only & 0.8520 & 0.0000 & $<0.0001$ \\
\hline Ln(AADT) & 0.6039 & 0.2912 & $<0.0001$ \\
\hline Minor commercial driveway density & 0.8012 & 0.0596 & $<0.0001$ \\
\hline Roadside object density & 0.8031 & 0.0574 & $<0.0001$ \\
\hline Major commercial driveway density & 0.8236 & 0.0333 & $<0.0001$ \\
\hline Speed limit & 0.8366 & 0.0181 & $<0.0001$ \\
\hline Presence of on-street parking & 0.8424 & 0.0113 & 0.0001 \\
\hline Presence of lighting & 0.8435 & 0.0100 & $<0.0001$ \\
\hline Minor residential driveway density & 0.8490 & 0.0035 & 0.1240 \\
\hline Minor industrial driveway density & 0.8492 & 0.0033 & 0.1830 \\
\hline Major industrial driveway density & 0.8500 & 0.0023 & 0.2800 \\
\hline Use of automated speed enforcement & 0.8517 & 0.0004 & 0.5160 \\
\hline Other driveway density & 0.8520 & 0.0000 & 0.4420 \\
\hline Major residential driveway density & 0.8520 & 0.0000 & 0.5950 \\
\hline
\end{tabular}

TABLE 6-3 NB Model Results for Urban and Suburban Three-Lane Arterials with a TWLTL

\begin{tabular}{|l|c|c|c|}
\hline Variable & $\boldsymbol{k}$ & $\boldsymbol{R}_{\boldsymbol{k}}^{2}$ & p-value \\
\hline Intercept-only & 0.9432 & 0.0000 & $<0.0001$ \\
\hline Ln(AADT) & 0.6662 & 0.2937 & $<0.0001$ \\
\hline Minor commercial driveway density & 0.7988 & 0.1531 & $<0.0001$ \\
\hline Roadside object density & 0.9065 & 0.0389 & 0.0027 \\
\hline Major commercial driveway density & 0.9093 & 0.0359 & 0.0212 \\
\hline Presence of on-street parking & 0.9285 & 0.0156 & 0.1360 \\
\hline Major residential driveway density & 0.9289 & 0.0152 & 0.0627 \\
\hline Presence of lighting & 0.9378 & 0.0057 & 0.1370 \\
\hline Minor residential driveway density & 0.9424 & 0.0008 & 0.5570 \\
\hline Minor industrial driveway density & 0.9425 & 0.0007 & 0.7400 \\
\hline Major industrial driveway density & 0.9428 & 0.0004 & 0.5700 \\
\hline Other driveway density & 0.9429 & 0.0003 & 0.3020 \\
\hline
\end{tabular}




\begin{tabular}{|l|c|c|c|}
\hline Variable & $\boldsymbol{k}$ & $\boldsymbol{R}_{\boldsymbol{k}}^{\mathbf{2}}$ & p-value \\
\hline Speed limit & 0.9433 & -0.0001 & 0.9020 \\
\hline Use of automated speed enforcement $^{*}$ & -- & -- & -- \\
\hline
\end{tabular}

${ }^{*}$ None of the segments have automated speed enforcement.

TABLE 6-4 NB Model Results for Urban and Suburban Four-Lane Undivided Arterials

\begin{tabular}{|l|c|c|c|}
\hline Variable & $\boldsymbol{k}$ & $\boldsymbol{R}_{\boldsymbol{k}}^{2}$ & p-value \\
\hline Intercept-only & 0.7090 & 0.0000 & $<0.0001$ \\
\hline Ln(AADT) & 0.5579 & 0.2131 & $<0.0001$ \\
\hline Major residential driveway density & 0.6638 & 0.0638 & 0.0049 \\
\hline Minor commercial driveway density & 0.6842 & 0.0350 & 0.0216 \\
\hline Presence of on-street parking & 0.7005 & 0.0120 & 0.1220 \\
\hline Major commercial driveway density & 0.7025 & 0.0092 & 0.2260 \\
\hline Roadside object density & 0.7047 & 0.0061 & 0.3650 \\
\hline Minor industrial driveway density & 0.7064 & 0.0037 & 0.4770 \\
\hline Presence of lighting & 0.7069 & 0.0030 & 0.6180 \\
\hline Speed limit & 0.7086 & 0.0006 & 0.7080 \\
\hline Major industrial driveway density & 0.7088 & 0.0003 & 0.5830 \\
\hline Minor residential driveway density & 0.7088 & 0.0003 & 0.5890 \\
\hline Other driveway density & 0.7089 & 0.0001 & 0.8110 \\
\hline Use of automated speed enforcement & -- & -- & - \\
\hline
\end{tabular}

${ }^{*}$ None of the segments have automated speed enforcement.

TABLE 6-5 NB Model Results for Urban and Suburban Four-Lane Divided Arterials

\begin{tabular}{|l|c|c|c|}
\hline Variable & $\boldsymbol{k}$ & $\boldsymbol{R}_{\boldsymbol{k}}^{\mathbf{2}}$ & p-value \\
\hline Intercept-only & 0.9223 & 0.0000 & $<0.0001$ \\
\hline Ln(AADT) & 0.5998 & 0.3497 & $<0.0001$ \\
\hline Major commercial driveway density & 0.8574 & 0.0704 & $<0.0001$ \\
\hline Median width & 0.8787 & 0.0473 & $<0.0001$ \\
\hline Minor commercial driveway density & 0.8798 & 0.0461 & $<0.0001$ \\
\hline Roadside object density & 0.9091 & 0.0143 & $<0.0001$ \\
\hline Other driveway density & 0.9183 & 0.0043 & $<0.0001$ \\
\hline Presence of lighting & 0.9195 & 0.0030 & 0.0036 \\
\hline Use of automated speed enforcement & 0.9202 & 0.0023 & 0.0072 \\
\hline Major industrial driveway density & 0.9210 & 0.0014 & 0.0298 \\
\hline Speed limit & 0.9215 & 0.0009 & 0.0637 \\
\hline Major residential driveway density & 0.9218 & 0.0005 & 0.2630 \\
\hline Presence of on-street parking & 0.9218 & 0.0005 & 0.2600 \\
\hline Minor residential driveway density & 0.9223 & 0.0000 & 0.8640 \\
\hline Minor industrial driveway density & 0.9223 & 0.0000 & 0.7920 \\
\hline
\end{tabular}


TABLE 6-6 NB Model Results for Urban and Suburban Five-Lane Arterials with a TWLTL

\begin{tabular}{|l|c|c|c|}
\hline Variable & $\boldsymbol{k}$ & $\boldsymbol{R}_{\boldsymbol{k}}^{2}$ & p-value \\
\hline Intercept-only & 0.7977 & 0.0000 & $<0.0001$ \\
\hline Ln(AADT) & 0.5640 & 0.2930 & $<0.0001$ \\
\hline Major commercial driveway density & 0.7118 & 0.1077 & $<0.0001$ \\
\hline Minor commercial driveway density & 0.7556 & 0.0528 & $<0.0001$ \\
\hline Minor residential driveway density & 0.7823 & 0.0193 & $<0.0001$ \\
\hline Presence of lighting & 0.7882 & 0.0119 & 0.0012 \\
\hline Roadside object density & 0.7894 & 0.0104 & 0.0028 \\
\hline Use of automated speed enforcement & 0.7951 & 0.0033 & 0.0624 \\
\hline Speed limit & 0.7955 & 0.0028 & 0.0822 \\
\hline Minor industrial driveway density & 0.7962 & 0.0019 & 0.1670 \\
\hline Major industrial driveway density & 0.7968 & 0.0011 & 0.1890 \\
\hline Major residential driveway density & 0.7969 & 0.0010 & 0.2640 \\
\hline Other driveway density & 0.7977 & 0.0000 & 0.9540 \\
\hline Presence of on-street parking ${ }^{*}$ & -- & -- & -- \\
\hline
\end{tabular}

${ }^{*}$ None of the segments have on-street parking.

\subsection{RF Models}

In order to develop an RF model that exhibits a better performance, the following two parameters were investigated with different sets of trials: the number of trees in the forest $\left(n_{\text {tree }}\right)$ and the number of predictor variables randomly selected for splitting each node $\left(m_{\text {try }}\right)$. Liaw and Wiener $(2002)$ suggested fitting a good number of trees $\left(n_{\text {tree }}\right)$ to obtain stable estimates of variable importance. Breiman (2002) suggested choosing the best $m_{\text {try }}$ setting from the following three different settings:

(a) $m_{\text {try }}$ equal to one-third of the number of predictor variables,

(b) $m_{\text {try }}$ equal to half of $m_{\text {try }}$ at (a), or

(c) $m_{\text {try }}$ equal to two times $m_{\text {try }}$ at (a).

The RF models for each roadway type were, therefore, developed with three values of $m_{\text {try }}$ for each of the $n_{\text {tree }}$ values equal to $500 ; 1,000 ; 5,000$; and 10,000 . The randomForest package of the statistical software $\boldsymbol{R}$ was used to build the RF models ( $\mathrm{R}$ 
Core Team, 2014). Other parameters were set as default, which included bootstrapping of data with test data size equal to 0.368 times the total number of observations, and allowing trees to grow with maximum possible number of terminal nodes.

\subsubsection{Optimization of RF Parameters}

Table 6-7 shows the performance measures of the RF models for different combinations of $n_{\text {tree }}$ and $m_{\text {try }}$ parameters. The performance is based on mean of squared residuals and percent variance explained. The measures were computed using test data set, typically referred to as out-of-bag (OOB) sample. An OOB sample is comprised of those observations that are not included in the bootstrap training data. The mean of squared residuals and the percent variance explained were estimated using the following equations (Liaw and Wiener, 2002):

$$
\begin{gathered}
M S R_{O O B}=\frac{1}{n} \sum_{i \in O O B}\left(y_{i}-\hat{y}_{i}\right)^{2} \\
\text { Percent Variance Explained }=1-\frac{M S R_{O O B}}{\hat{\sigma}_{y}^{2}}
\end{gathered}
$$

where,

$M S R_{O O B}=$ mean of squared residuals using OOB sample for observation $i$,

$y_{i}=$ observed value of the $i$ th observation in the OOB sample,

$\hat{y}_{i} \quad=$ predicted value of the $i$ th observation in the OOB sample,

$n \quad=$ number of observations in the OOB sample, and

$\hat{\sigma}_{y}^{2} \quad=$ variance of the response variable $y$ computed as $\frac{1}{n} \sum_{i \in O O B}\left(y_{i}-\bar{y}\right)^{2}$.

A lower value of mean squared residuals and, at the same time, a higher value of percent variance explained are attributed to a better performance of the RF models. As 
can be observed from Table 6-7, the value of $n_{\text {tree }}$ equal to 10,000 and $m_{\text {try }}$ equal to 4 had the lowest value of mean squared residuals and the highest value of percent variance explained for models of two-lane undivided arterials, four-lane undivided roads, four-lane divided roads, and five-lane arterials with a TWLTL. For three-lane arterials with a TWLTL, the model with $n_{\text {tree }}$ equal to 10,000 and $m_{\text {try }}$ equal to 8 was observed to perform better than other model settings. The importance of the variables was, therefore, estimated using the above mentioned values of the parameters for each corresponding roadway segment type.

TABLE 6-7 Performance of RF Models Developed for Urban and Suburban Arterial Segments

\begin{tabular}{|c|c|c|c|c|}
\hline Site Type & $\begin{array}{l}\text { Number of } \\
\text { Trees } \\
\left(n_{\text {tree }}\right)\end{array}$ & $\begin{array}{c}\text { Number of } \\
\text { Variables } \\
\text { Randomly Selected } \\
\left(m_{t r y}\right)\end{array}$ & $\begin{array}{l}\text { Mean of } \\
\text { Squared } \\
\text { Residuals }\end{array}$ & $\begin{array}{l}\text { Percent of } \\
\text { Variance } \\
\text { Explained }\end{array}$ \\
\hline \multirow{12}{*}{$\begin{array}{l}\text { Two-lane } \\
\text { undivided }\end{array}$} & \multirow{3}{*}{500} & 4 & 8.15 & 19.00 \\
\hline & & 2 & 8.31 & 17.36 \\
\hline & & 8 & 8.22 & 18.30 \\
\hline & \multirow{3}{*}{1,000} & 4 & 8.18 & 18.74 \\
\hline & & 2 & 8.31 & 17.42 \\
\hline & & 8 & 8.18 & 18.67 \\
\hline & \multirow{3}{*}{5,000} & 4 & 8.15 & 18.97 \\
\hline & & 2 & 8.27 & 17.76 \\
\hline & & 8 & 8.16 & 18.86 \\
\hline & \multirow{3}{*}{10,000} & 4 & 8.14 & 19.09 \\
\hline & & 2 & 8.29 & 17.65 \\
\hline & & 8 & 8.18 & 18.69 \\
\hline \multirow{12}{*}{$\begin{array}{c}\text { Three-lane with a } \\
\text { TWLTL }\end{array}$} & \multirow{3}{*}{500} & 4 & 16.72 & 28.37 \\
\hline & & 2 & 17.52 & 24.91 \\
\hline & & 8 & 16.98 & 27.24 \\
\hline & \multirow{3}{*}{1,000} & 4 & 16.64 & 28.68 \\
\hline & & 2 & 17.38 & 25.52 \\
\hline & & 8 & 16.81 & 27.99 \\
\hline & \multirow{3}{*}{5,000} & 4 & 16.64 & 28.69 \\
\hline & & 2 & 17.39 & 25.47 \\
\hline & & 8 & 16.55 & 29.08 \\
\hline & \multirow{3}{*}{10,000} & 4 & 16.59 & 28.93 \\
\hline & & 2 & 17.35 & 25.65 \\
\hline & & 8 & 16.51 & 29.25 \\
\hline
\end{tabular}




\begin{tabular}{|c|c|c|c|c|}
\hline Site Type & $\begin{array}{l}\text { Number of } \\
\text { Trees } \\
\left(n_{\text {tree }}\right)\end{array}$ & $\begin{array}{c}\text { Number of } \\
\text { Variables } \\
\text { Randomly Selected } \\
\left(m_{t r y}\right)\end{array}$ & $\begin{array}{l}\text { Mean of } \\
\text { Squared } \\
\text { Residuals }\end{array}$ & $\begin{array}{l}\text { Percent of } \\
\text { Variance } \\
\text { Explained }\end{array}$ \\
\hline \multirow{12}{*}{$\begin{array}{l}\text { Four-lane } \\
\text { undivided }\end{array}$} & \multirow{3}{*}{500} & 4 & 21.54 & 17.18 \\
\hline & & 2 & 22.18 & 14.75 \\
\hline & & 8 & 21.49 & 17.40 \\
\hline & \multirow{3}{*}{1,000} & 4 & 21.48 & 17.43 \\
\hline & & 2 & 21.92 & 15.74 \\
\hline & & 8 & 21.35 & 17.93 \\
\hline & \multirow{3}{*}{5,000} & 4 & 21.11 & 18.85 \\
\hline & & 2 & 21.70 & 16.57 \\
\hline & & 8 & 21.43 & 17.63 \\
\hline & \multirow{3}{*}{10,000} & 4 & 21.09 & 18.92 \\
\hline & & 2 & 21.64 & 16.80 \\
\hline & & 8 & 21.43 & 17.63 \\
\hline \multirow{12}{*}{ Four-lane divided } & \multirow{3}{*}{500} & 4 & 38.07 & 22.83 \\
\hline & & 2 & 38.15 & 22.67 \\
\hline & & 9 & 39.39 & 20.16 \\
\hline & \multirow{3}{*}{1,000} & 4 & 38.04 & 22.88 \\
\hline & & 2 & 38.08 & 22.81 \\
\hline & & 9 & 39.33 & 20.28 \\
\hline & \multirow{3}{*}{5,000} & 4 & 38.00 & 22.97 \\
\hline & & 2 & 38.08 & 22.81 \\
\hline & & 9 & 39.11 & 20.72 \\
\hline & \multirow{3}{*}{10,000} & 4 & 37.98 & 23.02 \\
\hline & & 2 & 38.08 & 22.81 \\
\hline & & 8 & 39.10 & 20.74 \\
\hline \multirow{12}{*}{$\begin{array}{c}\text { Five-lane with a } \\
\text { TWLTL }\end{array}$} & \multirow{3}{*}{500} & 4 & 44.69 & 24.43 \\
\hline & & 2 & 45.11 & 23.71 \\
\hline & & 8 & 44.66 & 24.47 \\
\hline & \multirow{3}{*}{1,000} & 4 & 44.69 & 24.42 \\
\hline & & 2 & 45.13 & 23.67 \\
\hline & & 8 & 44.67 & 24.45 \\
\hline & \multirow{3}{*}{5,000} & 4 & 44.54 & 24.67 \\
\hline & & 2 & 44.96 & 23.97 \\
\hline & & 8 & 44.88 & 24.11 \\
\hline & \multirow{3}{*}{10,000} & 4 & 44.57 & 24.63 \\
\hline & & 2 & 44.89 & 24.08 \\
\hline & & 8 & 44.94 & 24.00 \\
\hline
\end{tabular}

\subsubsection{Variable Importance by $R F$}

The variable importance in the RF models was measured by an increase in node purity values, which corresponds to a mean decrease in the residual sum of squares. 
Tables 6-8 through 6-12 show the rank of variables by node purity values for two-lane undivided arterials, three-lane arterials with a TWLTL, four-lane undivided arterials, four-lane divided arterials, and five-lane arterials with a TWLTL, respectively. The relative contribution of a variable was computed by dividing the respective node purity value by the sum of node purity values for all the variables.

In all the RF models, AADT is observed as the most influential variable with the highest value of increase in node purity. The roadside object density variable is ranked as the second most influential variable for two-lane undivided arterials, four-lane undivided roads, and four-lane divided roads; while it is ranked third for three-lane and five-lane arterials with a TWLTL.

Table 6-8 shows that minor commercial driveway density and minor residential driveway density are the next two influential variables AADT and roadside object density for two-lane undivided arterials. Among the other variables, presence of on-street parking, presence of lighting, major commercial driveway density, and major industrial driveway density each contributed at least $3 \%$ to estimate crash predictions.

As can be observed from Table 6-9, minor commercial driveway density is the second most influential variable followed by roadside object density for three-lane arterials with a TWLTL. The next two influential variables are major commercial driveway density and minor residential driveway density, each contributing between 5\% and $10 \%$. Among the remaining variables, only minor industrial driveway density had over $3 \%$ contributions in crash predictions for three-lane arterials with a TWLTL.

Table 6-10 shows that major residential driveway density and minor commercial driveway density are ranked third and fourth in the list of variable importance, where 
AADT and roadside object density are the top two most influential variables for four-lane undivided arterials. Cumulatively, these four variables contributed approximately $75 \%$ to the model fit. The variables minor residential driveway density and minor commercial driveway density each had good contributions (i.e., between $5 \%$ and $10 \%$ ), while each of the remaining variables except other driveway density showed approximately $2 \%$ to $3 \%$ contributions in developing the RF model for four-lane undivided arterials.

For four-lane divided roads (see Table 6-11), in addition to AADT and roadside object density, both the major and minor commercial driveway densities played a significant role in crash frequency predictions. The contributions by major and minor commercial driveway densities were similar, together accounting for approximately $25 \%$ of the total contribution. Median width is another variable that is observed to have a good contribution (i.e., greater than 5\%) to the model fit. The remaining nine variables contributed a total of less than $10 \%$ in developing the RF model.

For five-lane arterials with a TWLTL (see Table 6-12), it is evident that AADT, major commercial driveway density, roadside object density, and minor commercial driveway density showed substantial contributions compared to other variables. Cumulatively, these four variables accounted for approximately $93 \%$ of the total contribution. 
TABLE 6-8 Variable Importance by RF Model for Urban and Suburban Two-Lane Undivided Arterials

\begin{tabular}{|l|c|c|c|}
\hline Variable & $\begin{array}{c}\text { Increase in } \\
\text { Node Purity }\end{array}$ & $\begin{array}{c}\text { Relative } \\
\text { Contribution } \\
\mathbf{( \% )}\end{array}$ & $\begin{array}{c}\text { Cumulative } \\
\text { Contribution } \\
\mathbf{( \% )}\end{array}$ \\
\hline AADT & 4598.65 & 32.48 & 32.48 \\
\hline Roadside object density & 3912.58 & 27.63 & 60.11 \\
\hline Minor commercial driveway density & 1663.85 & 11.75 & 71.86 \\
\hline Minor residential driveway density & 1267.80 & 8.95 & 80.81 \\
\hline Presence of on-street parking & 525.94 & 3.71 & 84.52 \\
\hline Presence of lighting & 476.21 & 3.36 & 87.88 \\
\hline Major commercial driveway density & 440.02 & 3.11 & 90.99 \\
\hline Minor industrial driveway density & 424.43 & 3.00 & 93.99 \\
\hline Speed limit & 396.96 & 2.80 & 96.79 \\
\hline Other driveway density & 207.31 & 1.46 & 98.25 \\
\hline Major industrial driveway density & 144.94 & 1.02 & 99.27 \\
\hline Major residential driveway density & 95.80 & 0.68 & 99.95 \\
\hline Use of automated speed enforcement & 3.19 & 0.02 & 99.97 \\
\hline
\end{tabular}

TABLE 6-9 Variable Importance by RF Model for Urban and Suburban ThreeLane Arterials with a TWLTL

\begin{tabular}{|l|c|c|c|}
\hline Variable & $\begin{array}{c}\text { Increase in } \\
\text { Node Purity }\end{array}$ & $\begin{array}{c}\text { Relative } \\
\text { Contribution } \\
\mathbf{( \% )}\end{array}$ & $\begin{array}{c}\text { Cumulative } \\
\text { Contribution } \\
\text { (\%) }\end{array}$ \\
\hline AADT & 3159.70 & 41.15 & 41.15 \\
\hline Minor commercial driveway density & 1535.15 & 19.99 & 61.14 \\
\hline Roadside object density & 1068.39 & 13.91 & 75.05 \\
\hline Major commercial driveway density & 576.33 & 7.51 & 82.56 \\
\hline Minor residential driveway density & 428.97 & 5.59 & 88.15 \\
\hline Minor industrial driveway density & 279.29 & 3.64 & 91.79 \\
\hline Major residential driveway density & 185.25 & 2.41 & 94.20 \\
\hline Presence of lighting & 177.97 & 2.32 & 96.52 \\
\hline Speed limit & 84.33 & 1.10 & 97.62 \\
\hline Major industrial driveway density & 80.11 & 1.04 & 98.66 \\
\hline Presence of on-street parking & 64.79 & 0.84 & 99.50 \\
\hline Other driveway density & 38.19 & 0.50 & 100.00 \\
\hline $\begin{array}{l}\text { Use of automated speed } \\
\text { enforcement }\end{array}$ & -- & -- & - \\
\hline
\end{tabular}

${ }^{*}$ None of the segments have automated speed enforcement. 
TABLE 6-10 Variable Importance by RF Model for Urban and Suburban FourLane Undivided Arterials

\begin{tabular}{|l|c|c|c|}
\hline Variable & $\begin{array}{c}\text { Increase in } \\
\text { Node Purity }\end{array}$ & $\begin{array}{c}\text { Relative } \\
\text { Contribution } \\
\mathbf{( \% )}\end{array}$ & $\begin{array}{c}\text { Cumulative } \\
\text { Contribution } \\
\text { (\%) }\end{array}$ \\
\hline AADT & 1675.65 & 27.67 & 27.67 \\
\hline Roadside object density & 1146.48 & 18.93 & 46.60 \\
\hline Major residential driveway density & 904.95 & 14.94 & 61.54 \\
\hline Minor commercial driveway density & 796.45 & 13.15 & 74.69 \\
\hline Minor residential driveway density & 452.64 & 7.47 & 82.16 \\
\hline Minor industrial driveway density & 337.62 & 5.57 & 87.73 \\
\hline Major commercial driveway density & 181.06 & 2.99 & 90.72 \\
\hline Presence of on-street parking & 180.30 & 2.98 & 93.70 \\
\hline Major industrial driveway density & 151.23 & 2.50 & 96.20 \\
\hline Speed limit & 125.10 & 2.07 & 98.27 \\
\hline Presence of lighting & 103.81 & 1.71 & 99.98 \\
\hline Other driveway density & 1.11 & 0.02 & 100.00 \\
\hline $\begin{array}{l}\text { Use of automated speed } \\
\text { enforcement }\end{array}$ & - & -- & - \\
\hline None of the segments & - & & \\
\hline
\end{tabular}

${ }^{*}$ None of the segments have automated speed enforcement.

TABLE 6-11 Variable Importance by RF Model for Urban and Suburban FourLane Divided Arterials

\begin{tabular}{|l|c|c|c|}
\hline Variable & $\begin{array}{c}\text { Increase in } \\
\text { Node Purity }\end{array}$ & $\begin{array}{c}\text { Relative } \\
\text { Contribution } \\
\mathbf{( \% )}\end{array}$ & $\begin{array}{c}\text { Cumulative } \\
\text { Contribution } \\
\mathbf{( \% )}\end{array}$ \\
\hline AADT & 75356.89 & 40.36 & 40.36 \\
\hline Roadside object density & 37712.31 & 20.20 & 60.56 \\
\hline Major commercial driveway density & 23661.59 & 12.67 & 73.23 \\
\hline Minor commercial driveway density & 23457.72 & 12.56 & 85.79 \\
\hline Median width & 10826.94 & 5.80 & 91.59 \\
\hline Minor residential driveway density & 4475.94 & 2.40 & 93.99 \\
\hline Presence of lighting & 3510.19 & 1.88 & 95.87 \\
\hline Major residential driveway density & 2306.78 & 1.24 & 97.11 \\
\hline Use of automated speed enforcement & 1618.85 & 0.87 & 97.98 \\
\hline Major industrial driveway density & 1410.97 & 0.76 & 98.74 \\
\hline Speed limit & 1104.50 & 0.59 & 99.33 \\
\hline Minor industrial driveway density & 478.04 & 0.26 & 99.59 \\
\hline Presence of on-street parking & 443.74 & 0.24 & 99.83 \\
\hline Other driveway density & 326.78 & 0.18 & 100.01 \\
\hline
\end{tabular}


TABLE 6-12 Variable Importance by RF Model for Urban and Suburban FiveLane Arterials with a TWLTL

\begin{tabular}{|l|c|c|c|}
\hline Variable & $\begin{array}{c}\text { Increase in } \\
\text { Node Purity }\end{array}$ & $\begin{array}{c}\text { Relative } \\
\text { Contribution (\%) }\end{array}$ & $\begin{array}{c}\text { Cumulative } \\
\text { Contribution } \\
\text { (\%) }\end{array}$ \\
\hline AADT & 18398.67 & 34.69 & 34.69 \\
\hline Major commercial driveway density & 12099.93 & 22.81 & 57.50 \\
\hline Roadside object density & 9902.51 & 18.67 & 76.17 \\
\hline Minor commercial driveway density & 8917.04 & 16.81 & 92.98 \\
\hline Minor residential driveway density & 1409.21 & 2.66 & 95.64 \\
\hline Major residential driveway density & 556.36 & 1.05 & 96.69 \\
\hline Speed limit & 546.70 & 1.03 & 97.72 \\
\hline Presence of lighting & 533.23 & 1.01 & 98.73 \\
\hline Minor industrial driveway density & 476.76 & 0.90 & 99.63 \\
\hline Major industrial driveway density & 142.83 & 0.27 & 99.90 \\
\hline Use of automated speed enforcement & 48.17 & 0.09 & 99.99 \\
\hline Other driveway density & 7.96 & 0.02 & 100.01 \\
\hline Presence of on-street parking & -- & -- & -- \\
\hline
\end{tabular}

${ }^{*}$ None of the segments have on-street parking.

\subsection{BRT Models}

A series of BRT models were generated with combinations of shrinkage $(0.05$, $0.01,0.005,0.001$, and 0.0005$)$ and tree complexity $(1,5,10$, and 15$)$ values by fitting a total of 20,000 trees for each of the five urban and suburban arterial segment types. The BRT models were developed using the $g b m$ package of the statistical software $\boldsymbol{R}$ (R Core Team, 2014). The $g b m$ package provides the option to specify the distribution type for dependent variable. Poisson distribution was specified to build the BRT models for crash predictions.

Cross-validation $(\mathrm{CV})$ procedure was adopted for developing the BRT models. The CV procedure divides the full data set into $n$ mutually exclusive subsets, and fits the data $n$ times. In each run, a different subset is chosen as test data set while the remaining $(n-1)$ subsets are used to train the model. The model can learn better from data since all 
the observations in the data set are being used at some stage for training as well as for validation (De'ath, 2007; Elith et al., 2008). A 10-fold CV was used for selecting the optimal settings of regularization parameters. In gbm, stochasticity in BRT models is controlled by the bag.fraction parameter. A value of 0.50 was set as bag.fraction for random extraction of $50 \%$ of the training data without replacement from the full training set at each iteration. The stopping criterion for a node from splitting further was set such that terminal nodes have at least 10 observations.

\subsubsection{Optimization of BRT Parameters}

Figures 6-1 through 6-5 show the performance of the BRT models for all the five types of urban and suburban arterial sections. The curves in the figure (represented in black, red, green, blue, and cyan colors) depict how Poisson deviance is reduced over the ensemble of 20,000 trees with different sets of shrinkage factors $(0.05,0.01,0.005,0.001$, and 0.0005$)$ and tree complexities $(1,5,10$, and 15). The minimum Poisson deviance among different shrinkage factors is shown using the grey line at each tree complexity level. It is evident from the figures that smaller shrinkage factors $(0.001$ and 0.0005$)$ would require several thousands of trees to gradually converge to the minimum loss in Poisson deviance, whereas larger shrinkage factors $(0.05,0.01$, and 0.005$)$ would require fewer trees. Also, models underwent very abrupt reduction in deviance at shrinkage factor of 0.05 . Note that among all the models developed for three-lane arterials with a TWLTL and four-lane undivided arterials, the minimum Poisson deviance was achieved at tree complexity 5 and shrinkage factor 0.05 (see Figures 6-2 and 6-3). However, because of the rapid decrease in deviance, models fitted with these parameters could not always assure a better prediction performance. 
In general, relatively fewer trees were required to fit models with increasing tree complexities for the same shrinkage value. Models fitted with only the main effect of the variables (tree complexity level 1) had the highest deviance compared to the models fitted with variable interactions (tree complexity levels 5, 10, and 15). It means that decision trees with only two terminal nodes (tree complexity level 1) could not be able to explain the variability of the data well, even while fitting more trees. Models fitted with interaction among variables would be more effective in capturing the complexity of crash frequency data.

Table 6-13 presents the corresponding minimum values of Poisson deviance of all the BRT models developed for each urban and suburban arterial segment type. The digits in the parentheses show the number of trees at which the minimum deviance is achieved for the given set of shrinkage and tree complexity values. Elith et al. (2008) suggested fitting models with a minimum of 1,000 trees for stability. Therefore, even if a model fitted with fewer than 1,000 trees achieved the lowest deviance, it was not selected as the optimized model mainly because such a model might be quite unstable. 


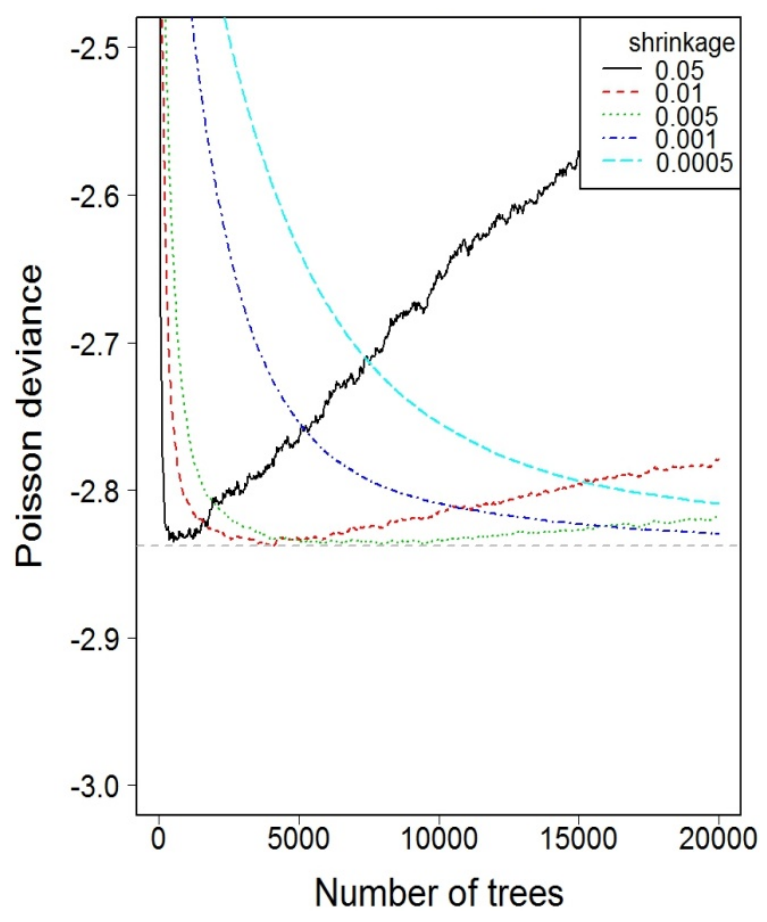

(a) Tree complexity: 1

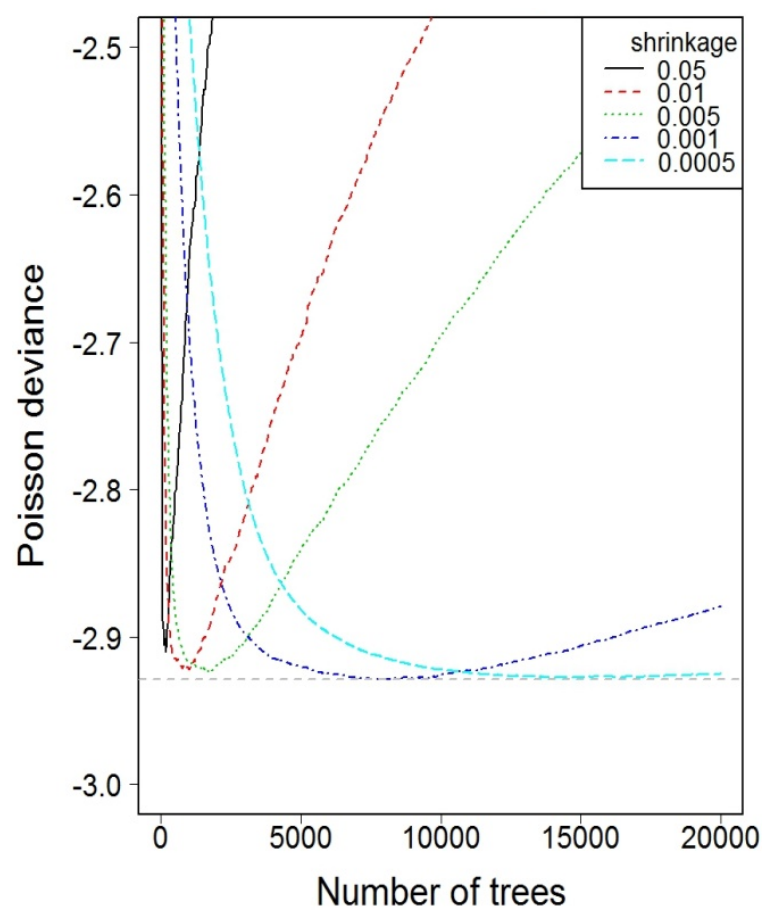

(c) Tree complexity: 10

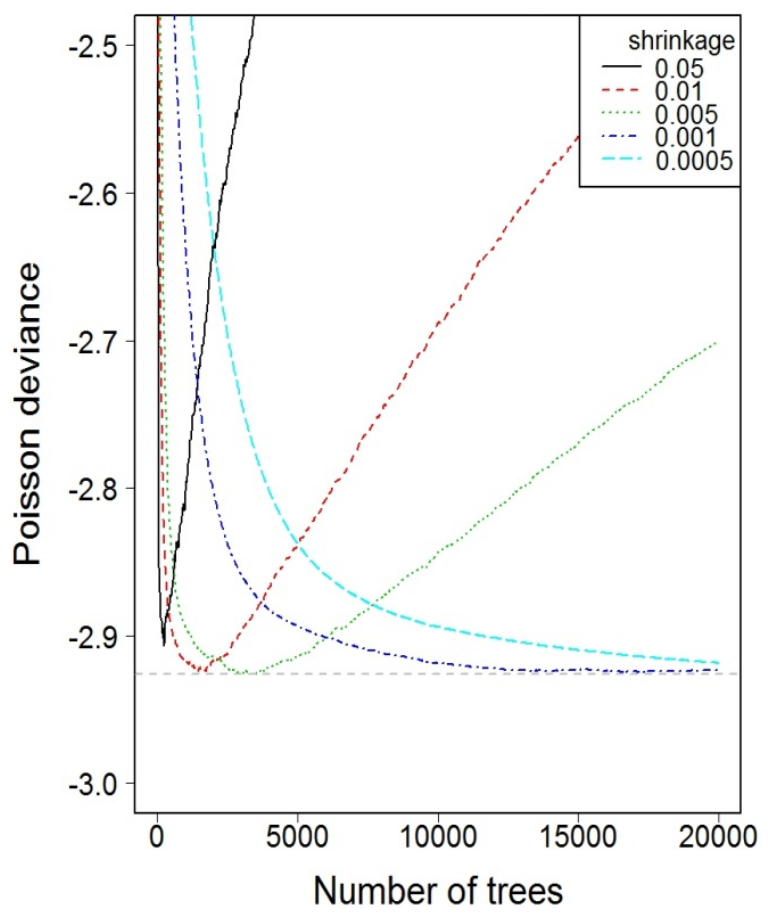

(b) Tree complexity: 5

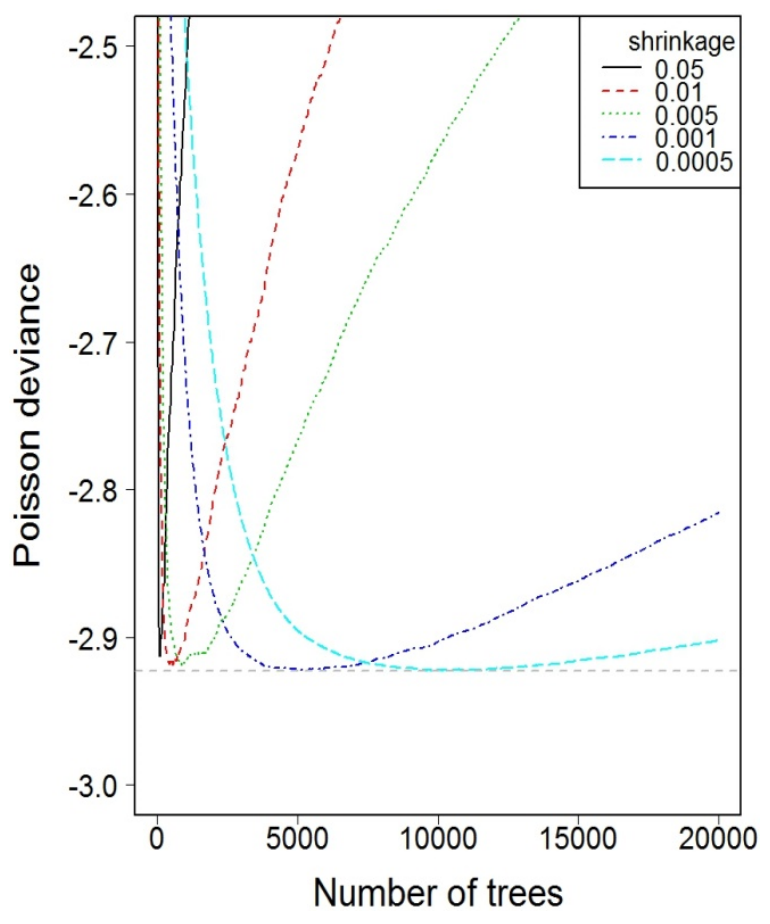

(d) Tree complexity: 15

FIGURE 6-1 Performance of BRT Models for Urban and Suburban Two-Lane Undivided Arterials 


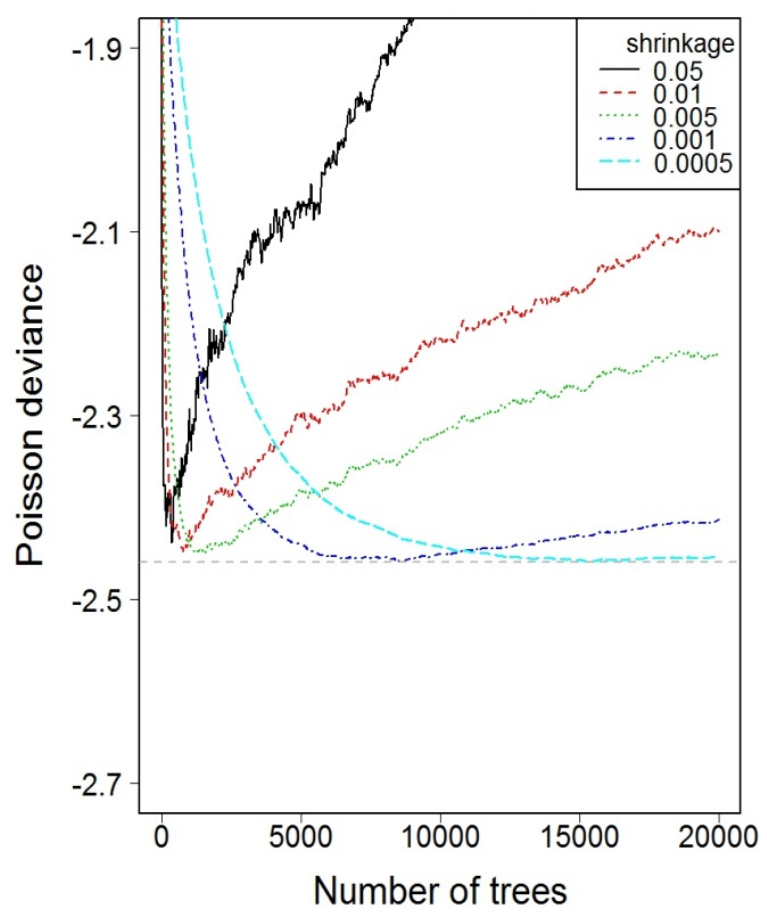

(a) Tree complexity: 1

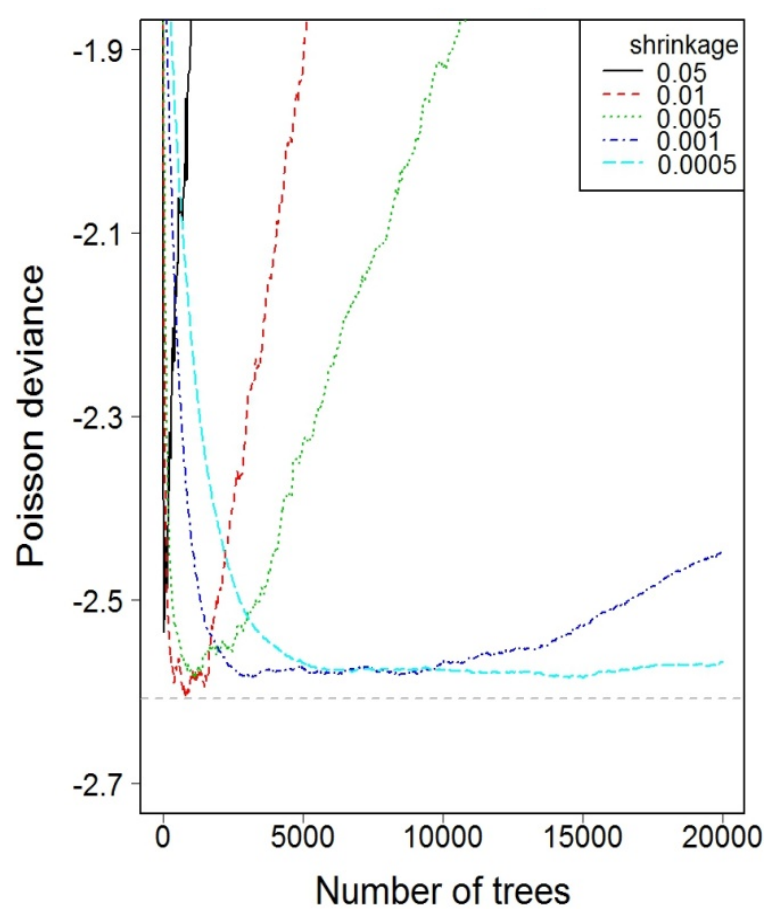

(c) Tree complexity: 10

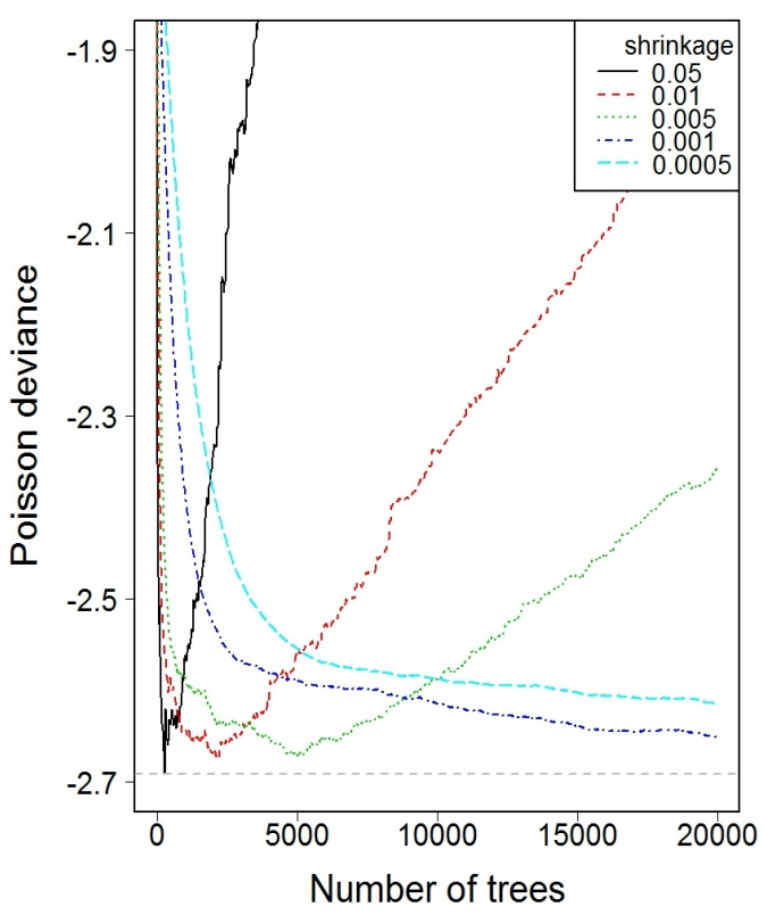

(b) Tree complexity: 5

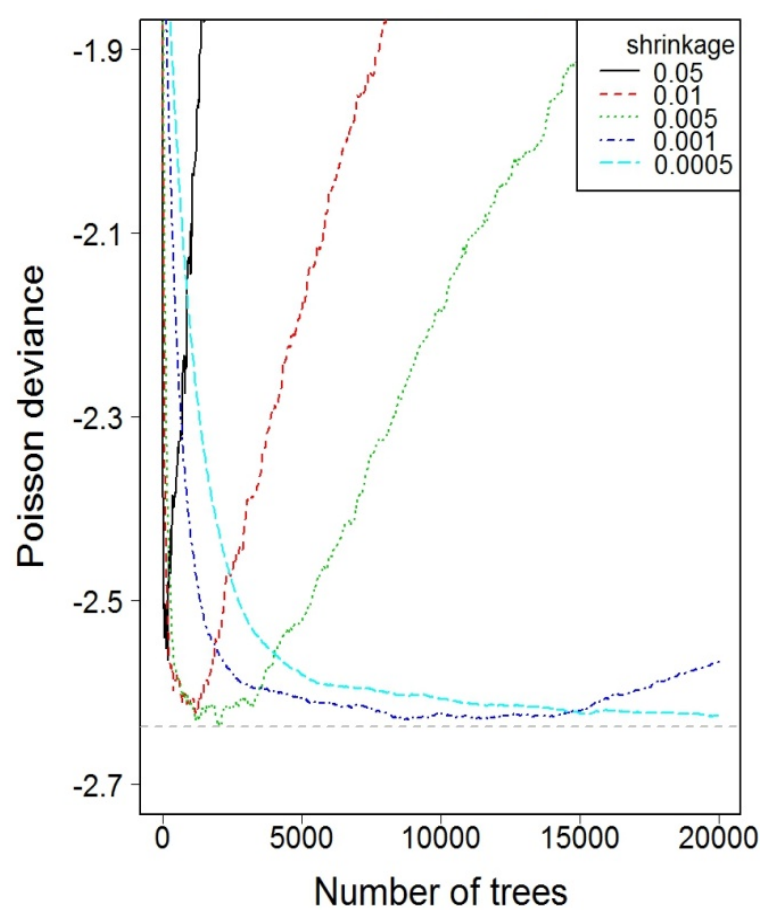

(d) Tree complexity: 15

FIGURE 6-2 Performance of BRT Models for Urban and Suburban Three-Lane Arterials with a TWLTL 


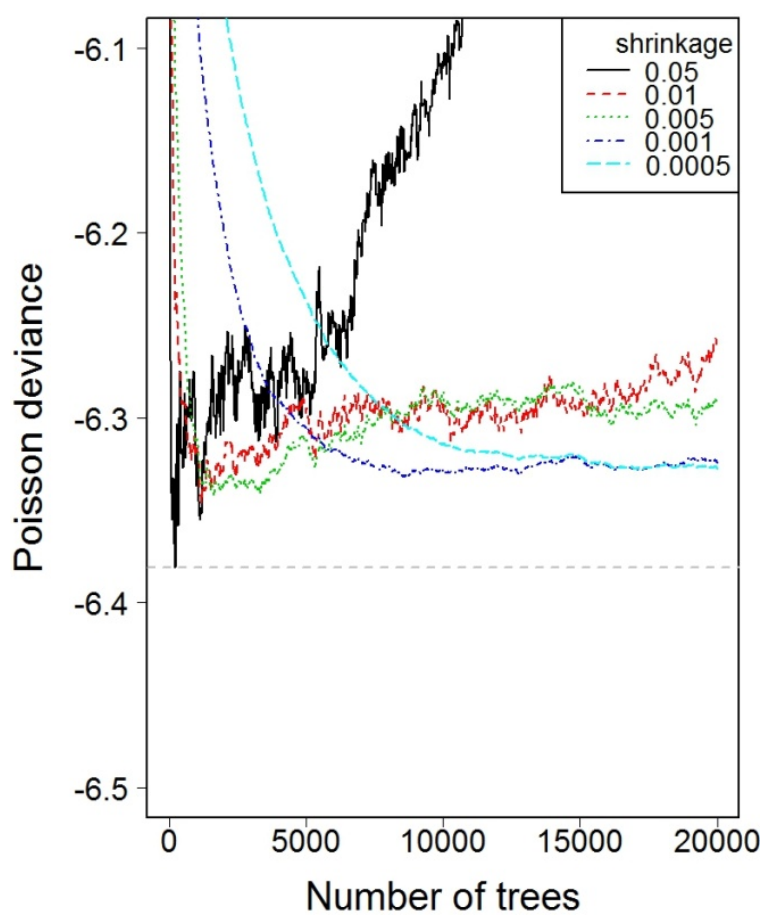

(a) Tree complexity: 1

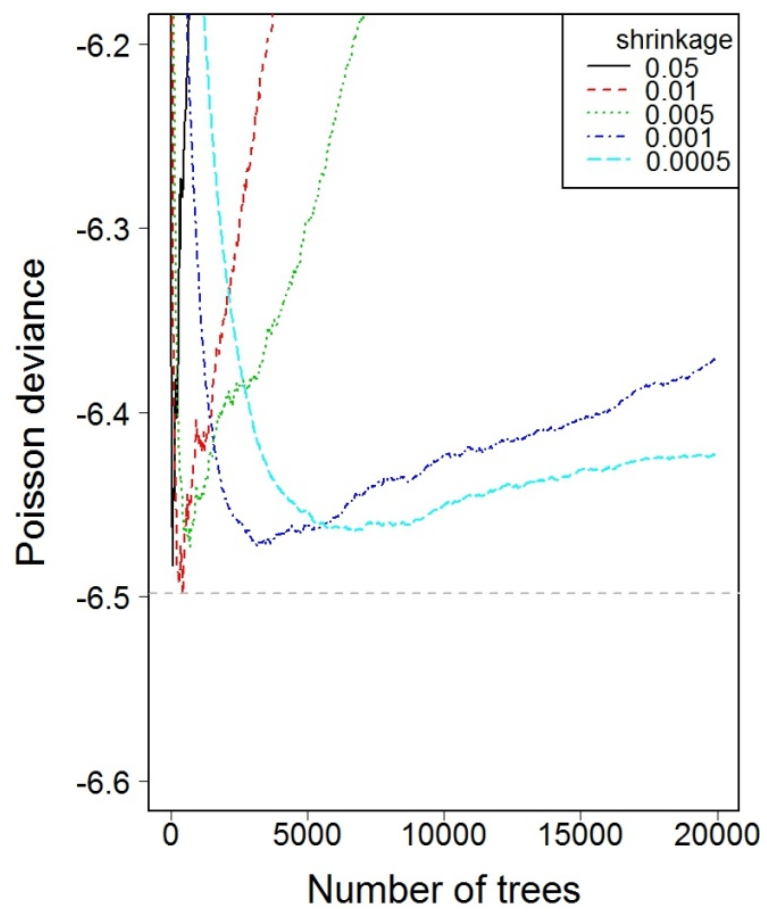

(c) Tree complexity: 10

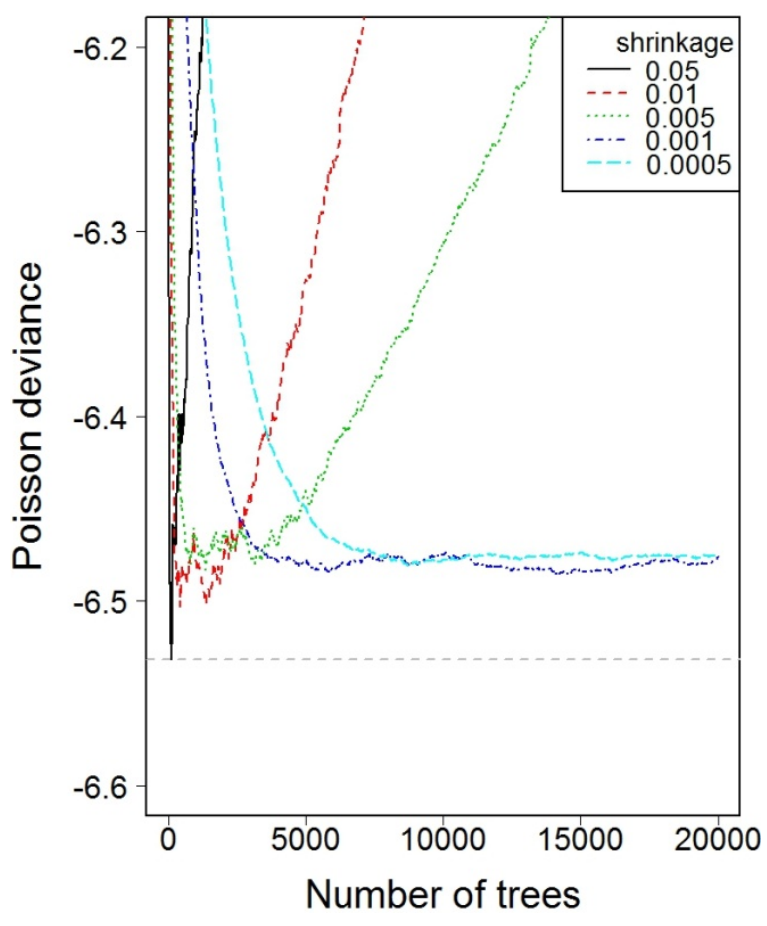

(b) Tree complexity: 5

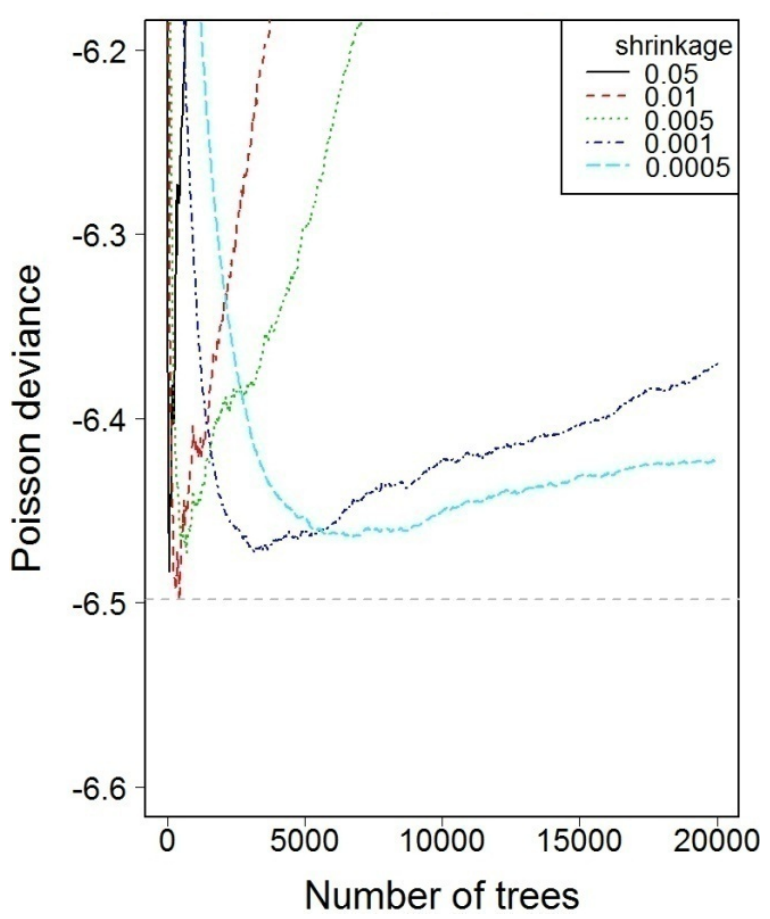

(d) Tree complexity: 15

FIGURE 6-3 Performance of BRT Models for Urban and Suburban Four-Lane Undivided Arterials 


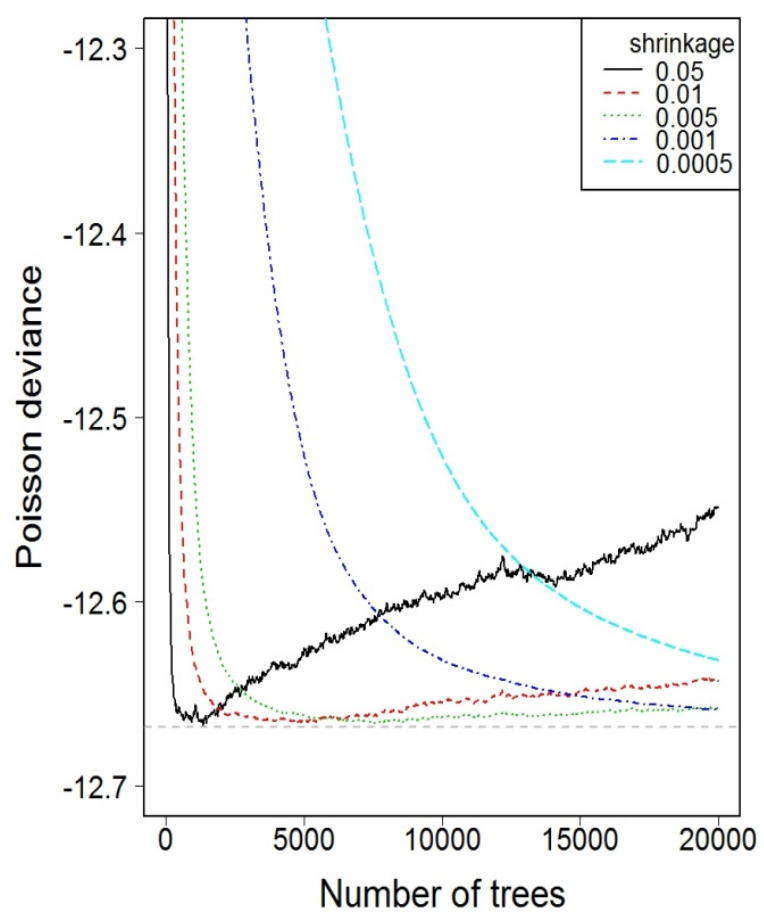

(a) Tree complexity: 1

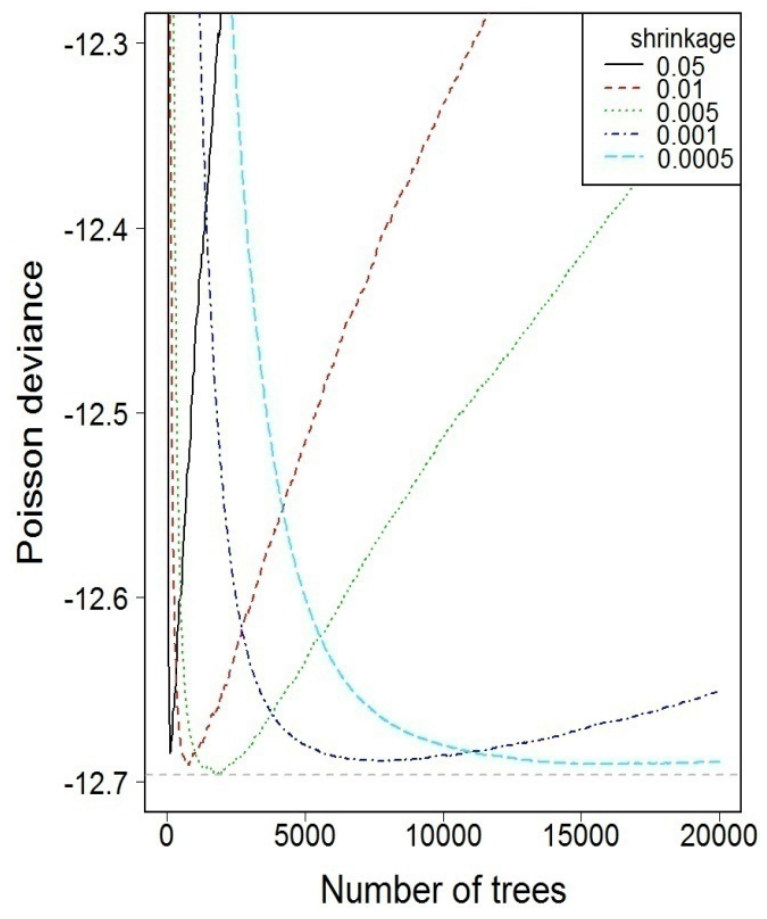

(c) Tree complexity: 10

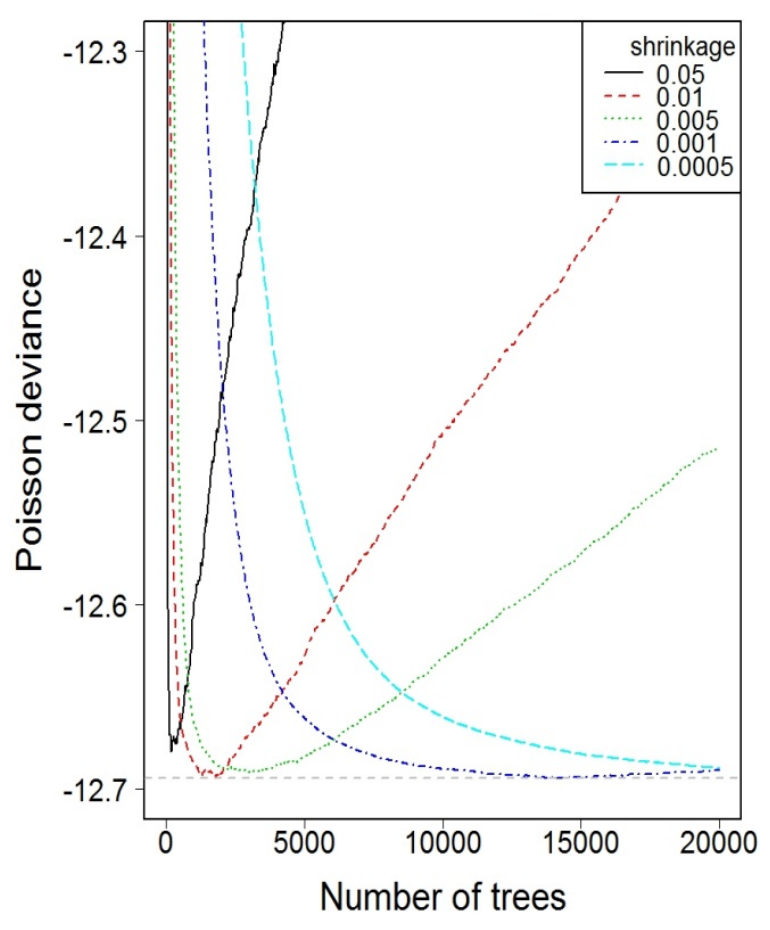

(b) Tree complexity: 5

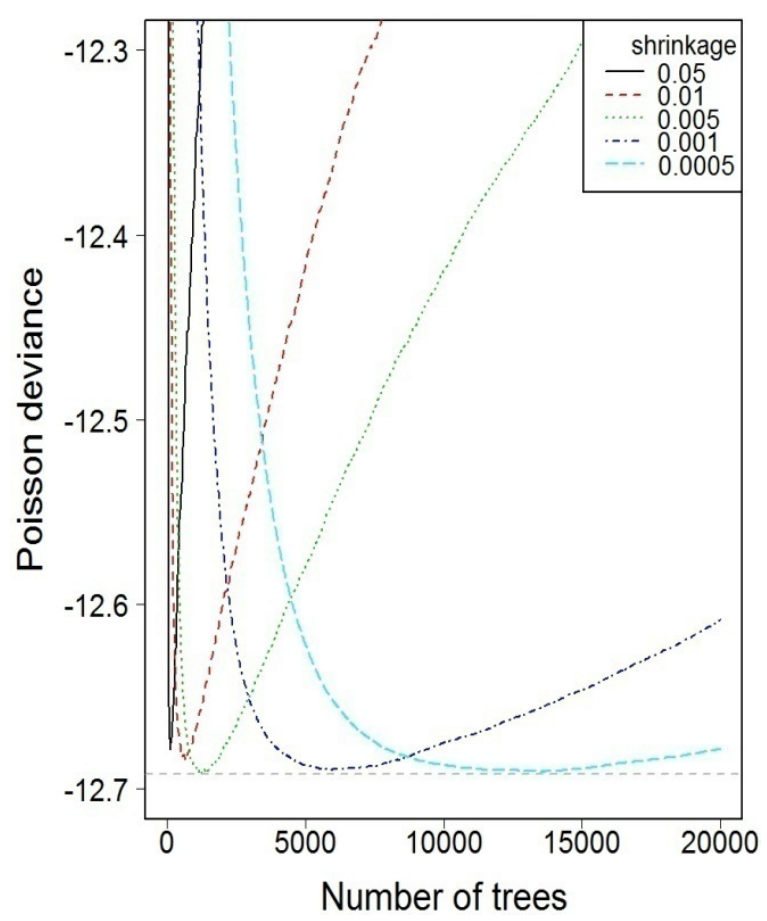

(d) Tree complexity: 15

FIGURE 6-4 Performance of BRT Models for Urban and Suburban Four-Lane Divided Arterials 


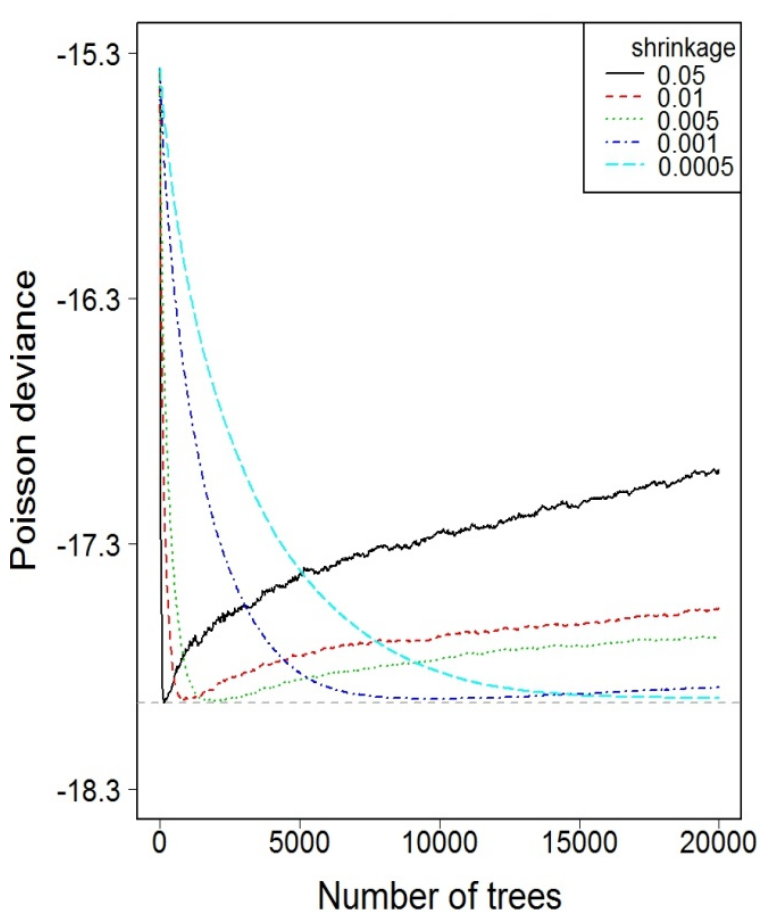

(a) Tree complexity: 1

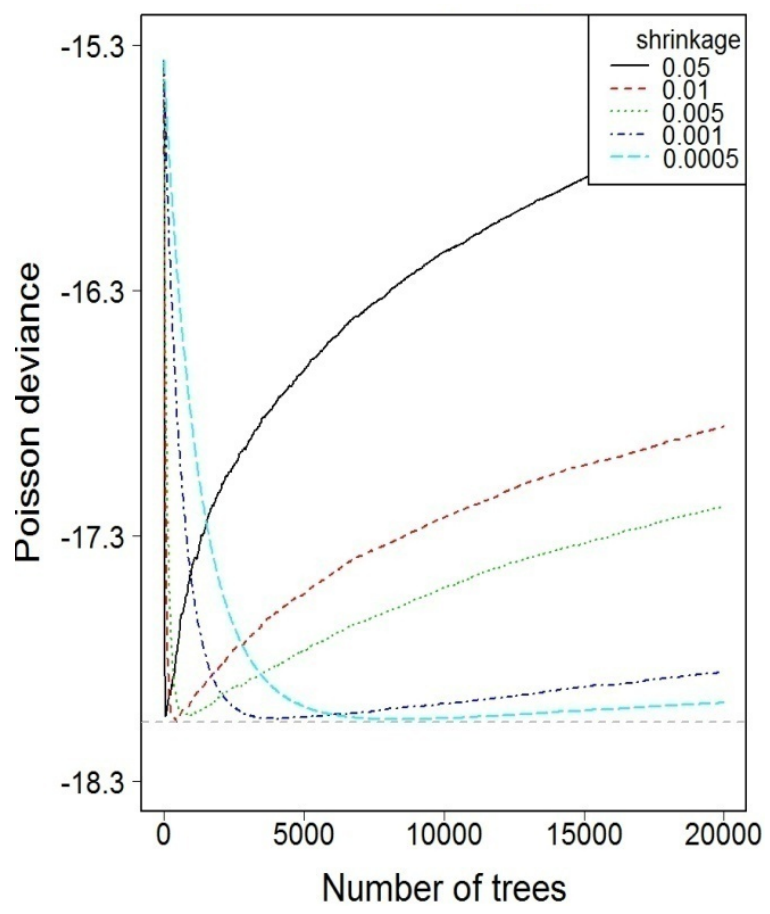

(c) Tree complexity: 10

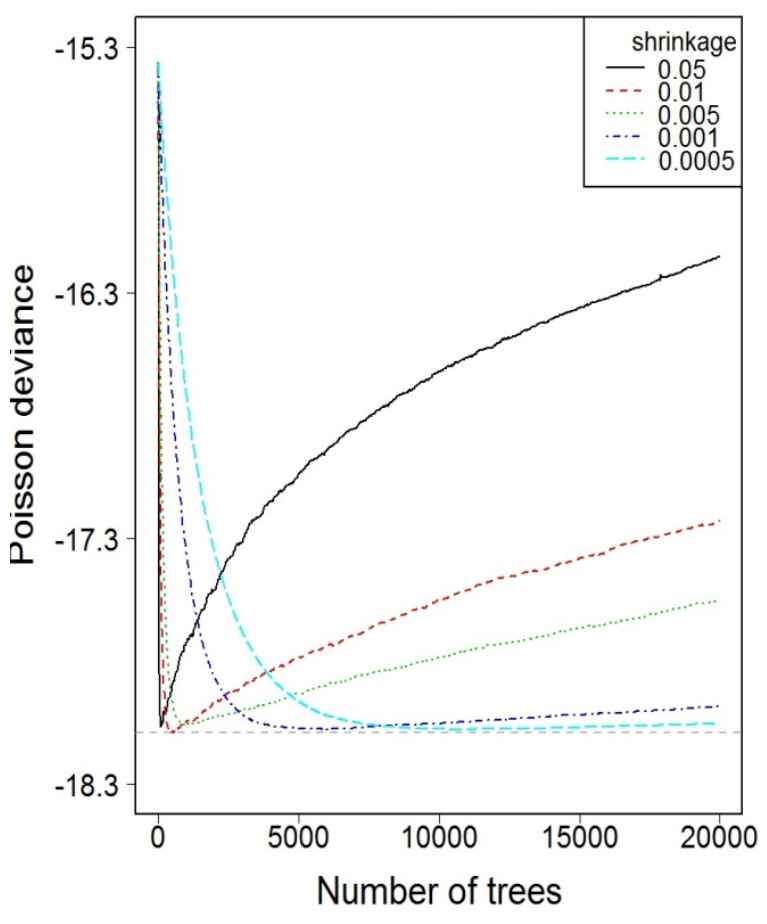

(b) Tree complexity: 5

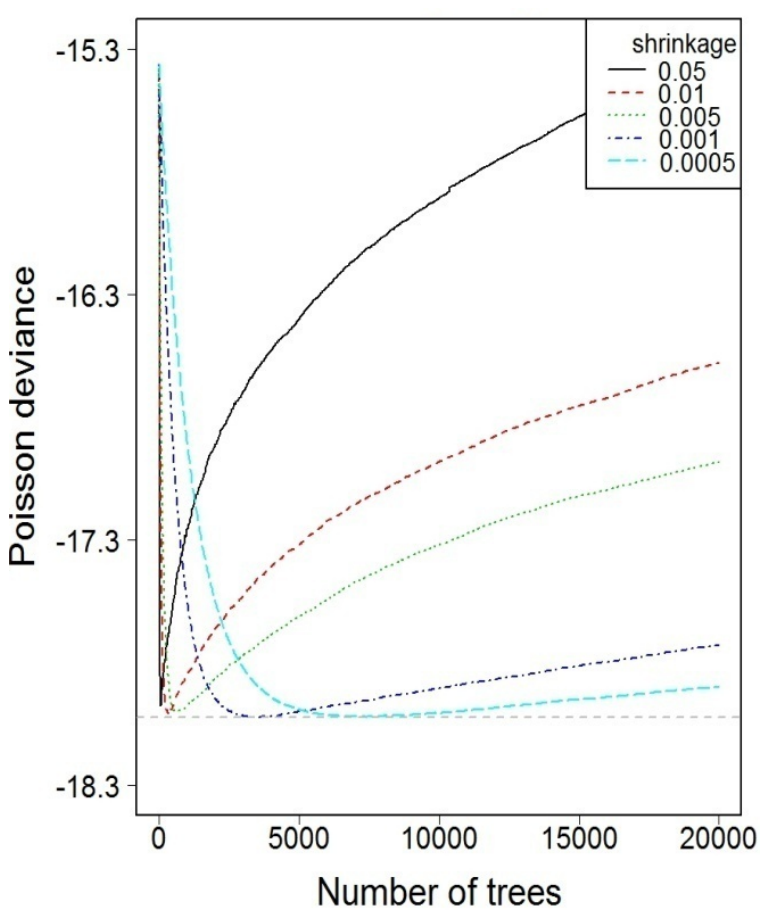

(d) Tree complexity: 15

FIGURE 6-5 Performance of BRT Models for Urban and Suburban Five-Lane Arterials with a TWLTL 
For two-lane undivided arterials, the minimum Poisson deviance is achieved at shrinkage factor of 0.001 , tree complexity of 10 and by fitting 7,983 trees. In the case of three-lane arterials with a TWLTL, models with tree complexity level 5 had significant reduction in deviance from models with tree complexity level 1 . The minimum deviation is observed at shrinkage factor of 0.05 and tree level 5. However, only 278 trees were fitted to obtain this deviance value illustrating a very sharp descend of the performance curve (see Figure 6-2). Therefore, the parameters were not considered to be optimized. Instead, it was found that lowering the shrinkage value to 0.01 at the same tree complexity could produce a stable model by fitting 2,180 trees. Again, models fitted for four-lane undivided arterials produced the lowest values of Poisson deviance while fitting fewer than 500 trees. When an ensemble of at least 1,000 trees is considered, the minimum loss in Poisson deviance is observed at shrinkage factor of 0.001 and tree complexity level 5. Note that higher level of tree complexity (i.e., $>10$ ) did not improve the model's performance for this roadway type. For four-lane divided roads, the lowest reduction in Poisson deviance occurred at tree complexity level 10 and shrinkage factor of 0.005 with an ensemble of 1,877 trees. The models for five-lane arterials with a TWLTL had the lowest values of Poisson deviance compared to models for other segment types. While the minimum value of Poisson deviance is found at shrinkage factor of 0.01 and tree complexity level 10, the model fitted fewer than 1,000 trees. A more stable model with lower deviance is obtained with an ensemble of 11,043 trees at shrinkage factor of 0.0005 and tree complexity level 5. It is also quite interesting to notice that none of the models with tree complexity level 15 performed better than models with tree complexity levels 5 and 10. 
TABLE 6-13 Minimum Poisson Deviance of BRT models for Urban and Suburban Arterial Segments

\begin{tabular}{|c|l|l|l|l|}
\hline \multirow{2}{*}{ Shrinkage Minimum Poisson Deviance (Optimum Number of Trees) } \\
\cline { 2 - 5 } & Tree complexity: 1 & Tree complexity: $\mathbf{5}$ & Tree complexity: 10 & Tree complexity: 15 \\
\hline \multicolumn{5}{|c|}{ Two-Lane Undivided } \\
\hline 0.05 & $-2.8352(515)$ & $-2.9065(233)$ & $-2.9103(176)$ & $-2.9125(97)$ \\
\hline 0.01 & $-2.8377(3,955)$ & $-2.9241(1,711)$ & $-2.9222(1,010)$ & $-2.9187(543)$ \\
\hline 0.005 & $-2.8366(7,946)$ & $-2.9262(3,317)$ & $-2.9239(1,734)$ & $-2.9194(884)$ \\
\hline 0.001 & $-2.8295(19,939)$ & $-2.9246(17,135)$ & $-2.9287(7,983)$ & $-2.9223(5,184)$ \\
\hline 0.0005 & $-2.8094(20,000)^{\mathrm{a}}$ & $-2.9185(19,998)^{\mathrm{a}}$ & $-2.9274(13,947)$ & $-2.9224(9,575)$ \\
\hline \multicolumn{5}{|c|}{ Three-Lane with a TWLTL } \\
\hline 0.05 & $-2.4376(373)$ & $-2.6910(278)$ & $-2.5643(193)$ & $-2.5349(39)$ \\
\hline 0.01 & $-2.4479(790)$ & $-2.6739(2,180)$ & $-2.6221(1,207)$ & $-2.6074(799)$ \\
\hline 0.005 & $-2.4508(1,309)$ & $-2.6709(5,015)$ & $-2.6369(2,035)$ & $-2.5859(1,138)$ \\
\hline 0.001 & $-2.4577(8,476)$ & $-2.6506(19,964)$ & $-2.6294(8,721)$ & $-2.5838(3,230)$ \\
\hline 0.0005 & $-2.4590(15,453)$ & $-2.6144(19,960)$ & $-2.6257(19,701)$ & $-2.5843(15,013)$ \\
\hline \multicolumn{5}{|c|}{ Four-Lane Undivided } \\
\hline 0.05 & $-6.3807(243)$ & $-6.5318(119)$ & $-6.4832(77)$ & $-6.4832(77)$ \\
\hline 0.01 & $-6.3466(1,187)$ & $-6.5030(422)$ & $-6.4981(426)$ & $-6.4981(426)$ \\
\hline 0.005 & $-6.3418(1,656)$ & $-6.4830(1,369)$ & $-6.4730(709)$ & $-6.4730(709)$ \\
\hline 0.001 & $-6.3324(8,717)$ & $-6.4852(14,509)$ & $-6.4725(3,200)$ & $-6.4725(3,200)$ \\
\hline 0.0005 & $-6.3280(17,272)$ & $-6.4809(8,725)$ & $-6.4643(6,697)$ & $-6.4643(6,697)$ \\
\hline \multicolumn{5}{|c|}{ Four-Lane Divided } \\
\hline 0.05 & $-12.6679(1,336)$ & $-12.6798(181)$ & $-12.6842(112)$ & $-12.6786(112)$ \\
\hline 0.01 & $-12.6661(4,723)$ & $-12.6936(1,915)$ & $-12.6906(786)$ & $-12.6836(650)$ \\
\hline 0.005 & $-12.6662(7,496)$ & $-12.6911(3,061)$ & $-12.6961(1,877)$ & $-12.6918(1,238)$ \\
\hline 0.001 & $-12.6588(19,988)^{*}$ & $-12.6940(14,118)$ & $-12.6889(7,596)$ & $-12.6896(5,870)$ \\
\hline 0.0005 & $-12.6317(20,000)^{*}$ & $-12.6885(19,996)^{*}$ & $-12.6904(15,307)$ & $-12.6906(13,627)$ \\
\hline \multicolumn{7}{|c|}{ Five-Lane with a TWLTL } \\
\hline 0.05 & $-17.9485(172)$ & $-18.0672(97)$ & $-18.0346(68)$ & $-17.9736(68)$ \\
\hline 0.01 & $-17.9374(810)$ & $-18.0901(479)$ & $-18.0568(443)$ & $-18.0073(328)$ \\
\hline 0.005 & $-17.9423(1,896)$ & $-18.0588(901)$ & $-18.0331(906)$ & $-17.9987(604)$ \\
\hline 0.001 & $-17.9325(9,580)$ & $-18.0771(5,936)$ & $-18.0456(4,064)$ & $-18.0239(3,437)$ \\
\hline 0.0005 & $-17.9274(19,691)$ & $-18.0774(11,043)$ & $-18.0476(8,962)$ & $-18.0210(7,411)$ \\
\hline
\end{tabular}

${ }^{*}$ Poisson deviance did not reach to its minimum value.

\subsubsection{Variable Importance by $B R T$}

The influence of a predictor variable in a BRT model was estimated by summing the squared improvements due to the splits by the variable over the ensemble of trees. The BRT models presented the influence of variables in a relative scale. Tables 6-14 through 6-18 show the influence of variables in the BRT models for two-lane undivided arterials, three-lane arterials with a TWLTL, four-lane undivided arterials, four-lane 
divided arterials, and five-lane arterials with a TWLTL, respectively. AADT, as expected, is the most influential variable in all the models. Among the other variables, roadside object density is observed to have greater than $10 \%$ contributions in all the models. It is also observed that the first three most influential variables in each model contributed to more than $75 \%$ of the total contribution.

Table 6-14 shows that in addition to AADT and roadside object density, minor residential driveway density had over $10 \%$ contributions to crash predictions for two-lane undivided arterials. The minor and major commercial driveway density and minor industrial driveway density are the next three influential variables, each with a contribution of $5 \%$ to $6 \%$ in fitting the BRT model. Major industrial driveway density, other driveway density, and presence of lighting had minimal influence (i.e., only slightly over $1 \%$ ) on crash predictions.

As shown in Table 6-15, minor commercial driveway density is the second most influential variable followed by roadside object density for three-lane arterials with a TWLTL. The variables minor residential driveway density, minor industrial driveway density, and major commercial driveway density each had approximately 5\% to $7 \%$ contributions in predicting crashes for this site type.

The three most influential variables for urban and suburban four-lane undivided roads (see Table 6-16) are AADT, roadside object density, and minor commercial driveway densities were similar, together accounting for approximately $14 \%$ of the total contribution. The remaining variables contributed a total of less than $10 \%$ of the total contribution. 
Table 6-17 shows that four variables including AADT, major commercial driveway density, roadside object density, and minor commercial driveway density each had more than $10 \%$ contributions on crash frequency predictions for four-lane divided arterials. Median width is another significant variable, with approximately $7.5 \%$ contributions. Minor residential driveway density had more than 3\% contributions while all the other variables together had less than $5 \%$ contributions.

Table 6-18 shows that the top four influential variables altogether contributed to greater than $95 \%$ in predicting crashes on five-lane arterials with a TWLTL. The variables are AADT, major commercial driveway density, roadside object density, and minor commercial driveway density. All the other variables together contributed less than $3 \%$ of the total contribution.

TABLE 6-14 Variable Importance by BRT Model for Urban and Suburban TwoLane Undivided Arterials

\begin{tabular}{|l|c|c|}
\hline Variable & $\begin{array}{c}\text { Relative Influence } \\
(\mathbf{\% )}\end{array}$ & $\begin{array}{c}\text { Cumulative Influence } \\
(\mathbf{\% )}\end{array}$ \\
\hline AADT & 44.62 & 44.62 \\
\hline Roadside object density & 22.10 & 66.72 \\
\hline Minor residential driveway density & 11.93 & 78.65 \\
\hline Minor commercial driveway density & 5.63 & 84.28 \\
\hline Major commercial driveway density & 5.07 & 89.35 \\
\hline Minor industrial driveway density & 4.76 & 94.11 \\
\hline Other driveway density & 1.64 & 95.75 \\
\hline Major industrial driveway density & 1.15 & 96.90 \\
\hline Presence of lighting & 1.00 & 97.90 \\
\hline Speed limit & 0.89 & 98.79 \\
\hline Presence of on-street parking & 0.86 & 99.65 \\
\hline Major residential driveway density & 0.35 & 100.00 \\
\hline Use of automated speed enforcement & 0.00 & 100.00 \\
\hline
\end{tabular}


TABLE 6-15 Variable Importance by BRT Model for Urban and Suburban ThreeLane Arterials with a TWLTL

\begin{tabular}{|l|c|c|}
\hline Variable & $\begin{array}{c}\text { Relative Influence } \\
(\mathbf{\% )}\end{array}$ & $\begin{array}{c}\text { Cumulative Influence } \\
\mathbf{( \% )}\end{array}$ \\
\hline AADT & 42.33 & 42.33 \\
\hline Minor commercial driveway density & 18.90 & 61.23 \\
\hline Roadside object density & 17.01 & 78.24 \\
\hline Minor residential driveway density & 7.03 & 85.27 \\
\hline Minor industrial driveway density & 6.33 & 91.60 \\
\hline Major commercial driveway density & 4.69 & 96.29 \\
\hline Presence of lighting & 1.84 & 98.13 \\
\hline Major residential driveway density & 1.16 & 99.29 \\
\hline Major industrial driveway density & 0.40 & 99.69 \\
\hline Speed limit & 0.31 & 100.00 \\
\hline Other driveway density & 0.00 & 100.00 \\
\hline Presence of on-street parking & 0.00 & 100.00 \\
\hline Use of automated speed enforcement & -- & --- \\
\hline
\end{tabular}

${ }^{*}$ None of the segments have automated speed enforcement.

TABLE 6-16 Variable Importance by BRT Model for Urban and Suburban FourLane Undivided Arterials

\begin{tabular}{|l|c|c|}
\hline Variable & $\begin{array}{c}\text { Relative Influence } \\
\text { (\%) }\end{array}$ & $\begin{array}{c}\text { Cumulative Influence } \\
\text { (\%) }\end{array}$ \\
\hline AADT & 37.27 & 37.27 \\
\hline Roadside object density & 27.57 & 64.84 \\
\hline Minor commercial driveway density & 12.51 & 77.35 \\
\hline Minor residential driveway density & 7.77 & 85.12 \\
\hline Minor industrial driveway density & 7.03 & 92.15 \\
\hline Presence of on-street parking & 2.42 & 94.57 \\
\hline Presence of lighting & 1.28 & 95.85 \\
\hline Major commercial driveway density & 1.18 & 97.03 \\
\hline Speed limit & 1.02 & 98.05 \\
\hline Major residential driveway density & 1.00 & 99.05 \\
\hline Major industrial driveway density & 0.94 & 99.99 \\
\hline Other driveway density & 0.00 & 99.00 \\
\hline Use of automated speed enforcement & -- & -- \\
\hline
\end{tabular}

None of the segments have automated speed enforcement. 
TABLE 6-17 Variable Importance by BRT Model for Urban and Suburban FourLane Divided Arterials

\begin{tabular}{|l|c|c|}
\hline Variable & $\begin{array}{c}\text { Relative Influence } \\
(\mathbf{\% )}\end{array}$ & $\begin{array}{c}\text { Cumulative Influence } \\
\text { (\%) }\end{array}$ \\
\hline AADT & 48.71 & 48.71 \\
\hline Major commercial driveway density & 13.57 & 62.28 \\
\hline Roadside object density & 12.07 & 74.35 \\
\hline Minor commercial driveway density & 10.29 & 84.64 \\
\hline Median width & 7.46 & 92.10 \\
\hline Minor residential driveway density & 3.52 & 95.62 \\
\hline Major residential driveway density & 1.79 & 97.41 \\
\hline Major industrial driveway density & 0.78 & 98.19 \\
\hline Minor industrial driveway density & 0.69 & 98.88 \\
\hline Presence of lighting & 0.61 & 99.49 \\
\hline Use of automated speed enforcement & 0.22 & 99.71 \\
\hline Presence of on-street parking & 0.16 & 99.87 \\
\hline Other driveway density & 0.09 & 99.96 \\
\hline Speed limit & 0.04 & 100.00 \\
\hline
\end{tabular}

TABLE 6-18 Variable Importance by BRT Model for Urban and Suburban FiveLane Arterials with a TWLTL

\begin{tabular}{|l|c|c|}
\hline Variable & $\begin{array}{c}\text { Relative Influence } \\
\text { (\%) }\end{array}$ & $\begin{array}{c}\text { Cumulative } \\
\text { Influence (\%) }\end{array}$ \\
\hline AADT & 39.14 & 39.14 \\
\hline Major commercial driveway density & 29.57 & 68.71 \\
\hline Roadside object density & 14.25 & 82.96 \\
\hline Minor commercial driveway density & 13.70 & 96.66 \\
\hline Minor residential driveway density & 1.15 & 97.81 \\
\hline Presence of lighting & 1.06 & 98.87 \\
\hline Minor industrial driveway density & 0.88 & 99.75 \\
\hline Major residential driveway density & 0.16 & 99.91 \\
\hline Major industrial driveway density & 0.08 & 99.99 \\
\hline Speed limit & 0.01 & 100.00 \\
\hline Other driveway density & 0.00 & 100.00 \\
\hline Use of automated speed enforcement & 0.00 & 100.00 \\
\hline Presence of on-street parking & -- & -- \\
\hline
\end{tabular}

${ }^{*}$ None of the segments have on-street parking. 


\subsection{Prioritization of Variables}

In this research, HSM calibration variables were prioritized using the following three methods: negative binomial (NB) regression, random forests (RF) and boosted regression trees (BRT). In the NB method, the influence of the variables was determined assuming a linear relationship between crash frequency and the associated factors, while in the RF and BRT methods, the variables were ranked with no such assumption of linearity between the dependent and independent variables. Although both the RF and BRT models determined variable importance by squared improvements due to the splits by a particular variable, the models had different processes including selecting a training data set, fitting the models, and combining the results of individual models. For example, the RF algorithm combines the results of individual models by giving equal weights to each model, while the BRT algorithm combines the results of individual models by giving more weights to weaker models. Again, the RF model randomly selects a subset of variables first and then determines the variable that could produce homogeneity for splitting a node, while the BRT model selects the variable for splitting a node from the full set of variables. Since the three methods use different approaches to prioritize variables, their results are given equal consideration in prioritizing the calibration variables.

Based on the empirical results of variable's influence on crash predictions, three criteria are set for grouping variables into variables of primary, secondary, or tertiary importance. The criteria are:

1. variables significant at 0.05 level in the NB model for crash predictions, 
2. variables with a minimum of $3 \%$ contributions in the $\mathrm{RF}$ model for crash predictions, and

3. variables with a minimum of $3 \%$ contributions in the BRT model for crash predictions.

The variables that satisfy all the above mentioned criteria are considered as variables of primary importance. The variables that satisfy only two of the three criteria are identified as variables of secondary importance. The variables that satisfy only one of the above criteria are considered as variables of tertiary importance. An example of how the variables are prioritized for two-lane undivided arterials is shown in Table 6-19. It is observed that AADT, major commercial driveway density, minor commercial driveway density, and roadside object density satisfy all the three criteria. Therefore, these variables are categorized as the variables of primary importance. Minor residential driveway density, minor industrial driveway density, presence of on-street parking, and presence of lighting are observed to fulfill two of the three criteria. Specifically, minor residential driveway density and minor industrial driveway density are found to have at least 3\% influence in both the RF and BRT models, presence of on-street parking and presence of lighting are found significant at $95 \%$ confidence level in the NB model and contributing more than $3 \%$ in the RF model. These four variables are, therefore, identified as variables of secondary importance. The speed limit variable satisfies only one of the three criteria and is, therefore, categorized as the variable of tertiary importance. All other variables in Table 6-19 show either relatively high p-values or relatively small contributions (i.e., approximately $3 \%$ or less) in both the RF and BRT models. 
TABLE 6-19 Variable Importance by NB, RF, and BRT Models for Urban and Suburban two-Lane Undivided Arterials

\begin{tabular}{|c|c|c|c|c|c|c|}
\hline \multirow{2}{*}{ Variable } & \multicolumn{2}{|c|}{ NB } & \multicolumn{2}{|l|}{ RF } & \multicolumn{2}{|c|}{ BRT } \\
\hline & p-value & Rank $^{1}$ & Rel. Cont. ${ }^{2}$ & Rank & Rel. Cont. ${ }^{2}$ & Rank \\
\hline $\mathrm{AADT}^{3}$ & $<0.0001$ & 1 & 32.48 & 1 & 44.62 & 1 \\
\hline $\begin{array}{l}\text { Major commercial driveway } \\
\text { density }^{3}\end{array}$ & $<0.0001$ & 4 & 3.11 & 7 & 5.07 & 5 \\
\hline Major residential driveway density & 0.5950 & 13 & 0.68 & 12 & 0.35 & 12 \\
\hline Major industrial driveway density & 0.1830 & 10 & 1.02 & 11 & 1.15 & 8 \\
\hline $\begin{array}{l}\text { Minor commercial driveway } \\
\text { density }^{3}\end{array}$ & $<0.0001$ & 2 & 11.75 & 3 & 5.63 & 4 \\
\hline Minor residential driveway density ${ }^{4}$ & 0.1240 & 8 & 8.95 & 4 & 11.93 & 3 \\
\hline Minor industrial driveway density ${ }^{4}$ & 0.1830 & 9 & 3.00 & 8 & 4.76 & 6 \\
\hline Other driveway density & 0.4420 & 11 & 1.46 & 10 & 1.64 & 7 \\
\hline Speed limit ${ }^{5}$ & $<0.0001$ & 5 & 2.80 & 9 & 0.89 & 10 \\
\hline Presence of on-street parking ${ }^{4}$ & 0.0001 & 7 & 3.71 & 5 & 0.86 & 11 \\
\hline Roadside object density $^{3}$ & $<0.0001$ & 3 & 27.63 & 2 & 22.10 & 2 \\
\hline Presence of lighting $^{4}$ & $<0.0001$ & 6 & 3.36 & 6 & 1.00 & 9 \\
\hline $\begin{array}{l}\text { Use of automated speed } \\
\text { enforcement }\end{array}$ & 0.5160 & 12 & 0.02 & 13 & 0.00 & 13 \\
\hline
\end{tabular}

${ }^{1}$ Rank is based on $R_{k}^{2}$ value from Table 6-2.

${ }^{2}$ Rel. Cont. indicates relative contribution in percentage.

${ }^{3}$ Variables are of primary importance.

${ }^{4}$ Variables are of secondary importance.

${ }^{5}$ Variables are of tertiary importance.

Table 6-20 lists the variables of primary, secondary, and tertiary importance for all the urban and suburban arterial segment types. It shows that only a few variables are likely to have significant impacts on crash predictions. The list of variables of primary importance includes five variables for four-lane divided roads, and only four variables for two-lane undivided arterials, three-lane arterials with a TWLTL, and five-lane arterials with a TWLTL. All types of minor driveways are categorized as variables of either primary or secondary importance for two-lane undivided, three-lane arterials, and fourlane undivided roads. In the case of four-lane divided roads, although none of the 
variables are categorized as the variables of secondary importance, several variables are identified as the variables of tertiary importance.

\subsection{Summary}

In summary, negative binomial (NB) regression, random forests (RF), and boosted regression trees (BRT) methods were used to determine the impact of calibration variables on crash predictions. The variables were ranked based on $R_{k}^{2}$ values in the NB models, by increase in node purity values in the RF models, by percentage of relative contribution in the BRT models. The results from the three models were combined to categorize variables into variables of primary, secondary, and tertiary importance. Only a few variables were identified as influential in safety predictions. Among them, AADT was the most influential variable in all the site types. Roadside object density, minor residential driveway density, and minor commercial driveway density were also identified as influential variables. 
TABLE 6-20 Prioritization of Variables

\begin{tabular}{|c|c|c|c|}
\hline Site Type & $\begin{array}{l}\text { Variables of Primary } \\
\text { Importance }\end{array}$ & $\begin{array}{l}\text { Variables of Secondary } \\
\text { Importance }\end{array}$ & $\begin{array}{l}\text { Variables of Tertiary } \\
\text { Importance }\end{array}$ \\
\hline $\begin{array}{l}\text { Two-lane } \\
\text { undivided }\end{array}$ & $\begin{array}{l}\text { - AADT } \\
\text { - Roadside object density } \\
\text { - Minor commercial } \\
\text { driveway density } \\
\text { - Major commercial } \\
\text { driveway density }\end{array}$ & $\begin{array}{l}\text { - Minor residential } \\
\text { driveway density } \\
\text { - Presence of on-street } \\
\text { parking } \\
\text { - Presence of lighting } \\
\text { - Minor industrial } \\
\text { driveway density }\end{array}$ & - Speed limit \\
\hline $\begin{array}{l}\text { Three-lane } \\
\text { with a } \\
\text { TWLTL }\end{array}$ & $\begin{array}{l}\text { - } \text { AADT } \\
\text { - Minor commercial } \\
\text { driveway } \\
\text { - Roadside object density } \\
\text { - Major commercial } \\
\text { driveway density }\end{array}$ & $\begin{array}{l}\text { Minor residential } \\
\text { driveway density } \\
\text { - Minor industrial } \\
\text { driveway density }\end{array}$ & \\
\hline $\begin{array}{l}\text { Four-lane } \\
\text { undivided }\end{array}$ & $\begin{array}{l}\text { - AADT } \\
\text { - Minor commercial } \\
\text { driveway density }\end{array}$ & $\begin{array}{l}\text { - Roadside object density } \\
\text { - Major residential } \\
\text { driveway density } \\
\text { - Minor residential } \\
\text { driveway density } \\
\text { - Minor industrial } \\
\text { driveway density }\end{array}$ & \\
\hline $\begin{array}{l}\text { Four-lane } \\
\text { divided }\end{array}$ & $\begin{array}{l}\text { - } \text { AADT } \\
\text { - Major commercial } \\
\text { driveway density } \\
\text { - Roadside object density } \\
\text { - Median width } \\
\text { - Minor commercial } \\
\text { driveway density }\end{array}$ & & $\begin{array}{l}\text { - Minor residential } \\
\text { driveway density } \\
\text { - Other driveway density } \\
\text { - Presence of lighting } \\
\text { - Use of automated speed } \\
\text { enforcement } \\
\text { - Major industrial } \\
\text { driveway density }\end{array}$ \\
\hline $\begin{array}{l}\text { Five-lane } \\
\text { with a } \\
\text { TWLTL }\end{array}$ & $\begin{array}{l}\text { - AADT } \\
\text { - Major commercial } \\
\text { driveway density } \\
\text { - Roadside object density } \\
\text { - Minor commercial } \\
\text { driveway density }\end{array}$ & & $\begin{array}{l}\text { - Minor residential } \\
\text { driveway density } \\
\text { - Presence of lighting }\end{array}$ \\
\hline
\end{tabular}




\section{CHAPTER 7}

\section{CONCLUSIONS AND RECOMMENDATIONS}

\subsection{Summary and Conclusions}

The Highway Safety Manual (HSM), published in 2010, is the result of decadelong efforts to provide quantitative statistical tools for crash analysis. The manual aims to provide transportation professionals with a single platform for integrating highway safety into decision-making processes in planning, design, construction, operations, and maintenance. The implementation of the HSM requires calibration of the HSM-default predictive models to reflect local conditions. The manual recommends deriving calibration factors using a data set consisting of 30 to 50 sites that experience a minimum of 100 crashes per year. Also, the manual emphasizes updating calibration factors at least every two to three years and preferably on an annual basis. These general recommendations are primarily based on expert opinions rather than specific research findings. Furthermore, most agencies do not have data for many of the input variables recommended in the HSM. As such, the goal of this research was to determine the best way to meet the data needs affecting the calibration factor estimations for urban and suburban arterials on Florida's state highway system. The specific objectives of this dissertation research were to:

- evaluate the effect of sample sizes on the calibration factor estimates and determine minimum sample sizes,

- validate the recommended calibration factor update frequency, and 
- determine the influence of variables on crash predictions and to identify and prioritize the influential calibration variables.

The scope of this research was limited to the following urban and suburban roadway segment and intersections in Florida:

- urban and suburban two-lane undivided arterials,

- urban and suburban three-lane arterials with a TWLTL,

- urban and suburban four-lane undivided roads,

- urban and suburban four-lane divided roads,

- urban and suburban five-lane arterials with a TWLTL,

- urban and suburban three-leg stop-controlled intersections, and

- urban and suburban four-leg signalized intersections.

The data collection and preparation process is the key component in accomplishing the research objectives. The major hindrance in the data preparation process was the unavailability of several HSM variables in the roadway characteristics inventory (RCI) database, the most comprehensive database maintained by the Florida Department of Transportation (FDOT). Therefore, extensive data collection efforts were undertaken to collect information on more than 20 variables representing roadway geometry and intersection characteristics. A Google Maps application called Visual Roadway Inventory Collection System (VRICS) was used to collect data from over 2,000 miles of road network and over 1,000 intersections in urban and suburban areas (i.e., areas with more than 5,000 population) in Florida. In addition, crash data for the most recent eight years (2005-2012) were retrieved from the Unified Basemap Repository (UBR) system, and used in the analyses. 
A sensitivity analysis was conducted to evaluate the effect of sample size on the reliability of estimated calibration factors. The sensitivity was measured based on the probability that the calibration factor estimated using a sample data set would vary within $10 \%$ of the calibration factor computed using the entire data set. Two sets of calibration factors, one computed using one year of crash data and the other computed using three consecutive years of crash data, were compared to determine whether or not the number of years of crash data used in deriving calibration factors affect their reliability. In general, calibration factors estimated using three consecutive years of crash data required a relatively small sample size to produce reliable calibration factors compared to the calibration factors estimated using one year of crash data.

The results of the sensitivity analysis show that the generalized one-size-fits-all approach of using a sample size of 30-50 sites is not appropriate as different facility types require different sample sizes depending on several factors, such as the extent of data variability, population size, crash experience, etc., to estimate reliable calibration factors. The sample size required to obtain a reliable calibration factor was significantly greater than the recommended criterion of 30 to 50 sites with at least 100 crashes per year. In particular, sample sizes with a minimum of 350, 200, 150, 300, and 250 sites were found to be required for urban and suburban two-lane undivided arterials, three-lane arterials with a TWLTL, four-lane undivided arterials, four-lane divided arterials, and five-lane arterials with a TWLTL, respectively, to estimate reliable calibration factors. A minimum of 140 intersections were required to estimate reliable calibration factors for urban and suburban three-leg stop controlled intersections, while 100 intersections were required for urban and suburban four-leg signalized intersections. 
Next, a series of paired sample t-tests were conducted to validate the recommended frequency for updating calibration factors. The objective was to compare whether or not there is any statistically significant difference among the calibration factors computed annually, every two years, and every three years. The results of the paired t-tests show that calibration factors need to be updated annually for all the urban and suburban arterial segment and intersection types.

Another major task performed in this research is prioritization of the HSM data variables based on their impact on crash predictions. The HSM lists a number of variables for deriving calibration factors. It is likely that not all of the variables have the same impact on safety predictions. Also, most of the variables are not available in the states' databases. The process of collecting and maintaining all the data variables on the entire road network for the purpose of implementing the HSM is cost-prohibitive. Therefore, this dissertation identified the influential variables by measuring the influence of each predictor on crash predictions. The following three methods were applied to determine variable importance for urban and suburban arterial segments: negative binomial (NB) regression, random forests $(\mathrm{RF})$, and boosted regression trees (BRT). Three different methods were considered based on the assumption that each method might produce different results in determining the influence of each variable on crash predictions and is, therefore, likely to rank variables differently.

The NB regression technique was used to develop single-variable models. The variables significant at 0.05 level were identified as the influential variables. The performance of the NB models was measured by $R_{k}^{2}$ values. A higher value of $R_{k}^{2}$ indicates a better fit. A number of models using both RF and BRT techniques were 
calibrated to determine the optimal values of regularizations parameters that indicate prediction performance of the models. In the RF model, variables were ranked based on the increase in node purity values that represent variable's involvement in building the model. The relative contribution of each variable was then estimated as the ratio of the corresponding increase in node purity value to the total sum of increase in node purity values. In the BRT model, variables were ranked based on the given relative contribution measure. In both RF and BRT models, the variables that showed at least $3 \%$ of relative contribution were identified as influential variables in both RF and BRT models.

The results from these three methods were combined to categorize variables into three groups: variables of primary importance, variables of secondary importance, and variables of tertiary importance. The variables identified as influential in all three models were categorized as variables of primary importance. The variables identified as influential in any two models were categorized as variables of secondary importance. The variables identified as influential in only one model were categorized as variables of tertiary importance. A total of four variables were identified as variables of primary importance for two-lane undivided arterials, three-lane arterials with a TWLTL, and fourlane divided arterials and five variables for five-lane arterials with a TWLTL, whereas only two variables were identified as variables of primary importance for four-lane undivided arterials. A total of four variables were identified as variables of secondary importance for two-lane undivided arterials and four-lane undivided arterials, and only two variables for three-lane arterials with a TWLTL. Although none of the variables were categorized as variables of secondary importance for four-lane divided arterials and fivelane arterials with a TWLTL, five variables belonged to the category of variables of 
tertiary importance for four-lane divided arterials and two variables for five-lane arterials with a TWLTL. Only one variable was found to be of tertiary importance for two-lane undivided arterials.

Among all the variables, AADT is observed as the most influential variable for all the roadway segment types. Besides AADT, roadside object density, minor commercial driveway density, and minor residential driveway density are also identified as influential in all the segment types. It is worth mentioning that the HSM identifies roadside object density as a desirable variable based on the assumption that it does not significantly affect crash predictions; however, the results presented in this dissertation justify re-evaluating the data needs for this variable.

\subsection{Research Contributions}

Since the release of the HSM in 2010, state and local agencies have been struggling with its implementation. Meeting the data requirements is one of the most challenging tasks in implementing the HSM. This dissertation provides improved processes in achieving better model results with minimum data collection efforts such that the potential impacts to the quality of analysis is not compromised. In particular, this research provides insights on the impacts of number of sites, observed crash frequency, and use of one versus three years of data on estimating reliable calibration factors. Furthermore, this dissertation provides measures for identifying the most influential variables by taking into consideration the complex nature of crash data.

The research results provide guidance on the minimum sample sizes required to calibrate reliable safety performance functions. The research results also help agencies determine how frequently calibration factors should be updated. The guideline on ranking 
of data variables helps agencies prioritize the additional data needs. The prioritized list of data variables would streamline the agencies' efforts for collecting and maintaining data for continued updates.

\subsection{Recommendations for Future Research}

Future studies to extend this dissertation research could include:

1. The effect of sample size on calibration factors was investigated based on total crash frequency. Agencies might be interested in developing calibration factors separately for fatal-and-injury crashes and property damage only crashes, or for some specific crash types (e.g., vehicle-animal crashes). It is very likely that sample size requirements would vary for different circumstances. A future research could apply the sensitivity analysis procedure to determine the required sample size for specific crash severity levels and crash types.

2. The variable importance measures used in the dissertation determine the impact of variables on total crashes. It is likely that some variables might have less impact on total crashes, nonetheless more impact on specific crash types (for example, presence of red light cameras at intersections has more influence on rear-end and right-angle crashes compared to total crashes). Future research can extend using the measures to determine the influential variables specific to particular crash types and for different facility types. 


\section{REFERENCES}

Abdel-Aty, M. A., and A. E. Radwan, "Modeling Traffic Accident Occurrence and Involvement," Accident Analysis and Prevention, vol. 32, no. 5, pp. 633-642, 2000.

Abdel-Aty, M., and K. Haleem, "Analyzing Angle Crashes at Unsignalized Intersections using Machine Learning Techniques," Accident Analysis and Prevention, vol. 43, no. 1, pp. 461-470, 2011.

Akgüngör, A. P., and O. Y1ldız, "Sensitivity Analysis of an Accident Prediction Model by the Fractional Factorial Method," Accident Analysis and Prevention, vol. 39, no. 1, pp. 63-68, 2007.

Alluri, P., and J. Ogle, "Effects of State-Specific SPFs, AADT Estimations, and Overdispersion Parameters on Crash Predictions Using SafetyAnalyst," in Proceedings of the 91st Annual Meeting of the Transportation Research Board (CD-ROM), Washington, D.C., 2012.

Alluri, P., D. Saha, K. Liu, and A. Gan, "Improved Processes for Meeting the Data Requirements for Implementing the Highway Safety Manual (HSM) and SafetyAnalyst in Florida," Lehman Center for Transportation Research, Florida International University, Contract BDK80-977-37, Final Report Prepared for Florida Department of Transportation, 2014.

American Association of State Highway and Transportation Officials (AASHTO), Highway Safety Manual. Washington, D.C.: Transportation Research Board of the National Academies, 2010.

Banihashemi, M., "Highway Safety Manual, New Model Parameters vs. Calibration of Crash Prediction Models," in Proceedings of the 90th Annual Meeting of the Transportation Research Board (CD-ROM), Washington, D.C., 2011.

Banihashemi, M., "Sensitivity Analysis of Data Set Sizes for Highway Safety Manual Calibration Factors," Transportation Research Record: Journal of Transportation Research Board, no. 2279, pp. 75-81, 2012.

Breiman, L., "Bagging Predictors,” Machine Learning, vol. 24, no. 2, pp.123-140, 1996.

Breiman, L., "Random Forests,” Machine Learning, vol. 45, no. 1, pp. 5-32, 2001.

Breiman, L., "Manual on Setting up, Using, and Understanding Random Forests v3.1," 2002. http://oz.berkeley.edu/users/breiman/Using_random_forests_V3.1.pdf.

Breiman, L., J. H. Friedman, R. A. Olshen, and C. J. Stone, Classification and Regression Trees. New York: Chapman and Hall, 1984. 
Brimley, B. K., M. Saito, and G. G. Schultz, "Calibration of Highway Safety Manual Safety Performance Function: Development of New Models for Rural Two-Lane TwoWay Highways," Transportation Research Record: Journal of Transportation Research Board, no. 2279, pp. 82-89, 2012.

Cafiso, S., A. Di Graziano, G. Di Silvestro, G. La Cava, and B. Persaud, "Development of Comprehensive Accident Models for Two-Lane Rural Highways Using Exposure, Geometry, Consistency and Context Variables," Accident Analysis and Prevention, vol. 42, no. 4, pp. 1072-1079, 2010.

Caliendo, C., M. Guida, and A. Parisi, "A Crash-Prediction Model for Multilane Roads," Accident Analysis and Prevention, vol. 39, no. 4, pp. 657-670, 2007.

Chang, L.-Y., and H.-W. Wang, "Analysis of Traffic Injury Severity: An Application of Non-Parametric Classification Tree Techniques," Accident Analysis and Prevention, vol. 38, no. 5, pp. 1019-1027, 2006.

Cheong, Y. L., P. J. Leitão, and T. Lakes, "Assessment of Land Use Factors Associated with Dengue Cases in Malaysia Using Boosted Regression Trees," Spatial and SpatioTemporal Epidemiology, vol. 10, pp. 75-84, 2014.

Chrysostomou, K., S. Y. Chen, and X. Liu, "Identifying User Preferences with WrapperBased Decision Trees," Expert Systems with Applications, vol. 38, no. 4, pp. 3294-3303, Apr. 2011.

Chung, Y.-S., "Factor Complexity of Crash Occurrence: An Empirical Demonstration Using Boosted Regression Trees," Accident Analysis and Prevention, vol. 61, 2013, pp. $107-118,2013$.

Das, A., M. Abdel-Aty, and A. Pande, "Using Conditional Inference Forests to Identify the Factors Affecting Crash Severity on Arterial Corridors," Journal of Safety Research, vol. 40, no. 4, pp. 317-327, 2009.

De'ath, G., "Boosted Trees for Ecological Modeling and Prediction," Ecology, vol. 88, no. 1, pp. 243-251, 2007.

Dixon, K., C. Monsere, F. Xie, and K. Gladhill, "Calibrating the Future Highway Safety Manual Predictive Methods for Oregon State Highways," Oregon State University and Portland State University, Report No. FHWA-OR-RD-12-07, Final Report Prepared for Oregon Department of Transportation, 2012.

Easa, S. M., and Q. C. You, "Collision Prediction Models for Three-Dimensional TwoLane Highways," Transportation Research Record: Journal of Transportation Research Board, no. 2092, pp. 48-56, 2009.

Elith, J., J. R. Leathwick, and T. Hastie, "A Working Guide to Boosted Regression Trees," Journal of Animal Ecology, vol. 77, no. 4, pp. 802-813, 2008. 
Ellis, A. R., S. B. Dusetzina, R. A. Hansen, B. N. Gaynes, J. F. Farley, and T. Stürmer, "Confounding Control in a Nonexperimental Study of STAR*D Data: Logistic Regression Balanced Covariates Better than Boosted CART," Annals of Epidemiology, vol. 23, no. 4, pp. 204-209, 2013.

Elmitiny, N., X. Yan, E. Radwan, C. Russo, and D. Nashar, "Classification Analysis of Driver's Stop/Go Decision and Red-Light Running Violation," Accident Analysis and Prevention, vol. 42, no. 1, pp. 101-111, 2010.

Esther, A., C. Imholt, J. Perner, J. Schumacher, and J. Jacob, "Correlations Between Weather Conditions and Common Vole (Microtus Arvalis) Densities Identified by Regression Tree Analysis," Basic and Applied Ecology, vol. 15, no. 1, pp. 75-84, 2014.

Etter, A., C. McAlpine, K. Wilson, S. Phinn, and H. Possingham, "Regional Patterns of Agricultural Land Use and Deforestation in Colombia," Agriculture, Ecosystems and Environment, vol. 114, no. 2-4, pp. 369-386, 2006.

Federal Highway Administration (FHWA), Highway Functional Classification: Concepts, Criteria and Procedures (2013 edition), U. S. Department of Transportation, 2013.

URL: https://www.fhwa.dot.gov/planning/processes/statewide/related/highway functional clas sifications/fcauab.pdf. Accessed on April 10, 2014.

Findley, D., C. Zegeer, C. Sundstrom, J. Hummer, and W. Rasdorf, "Applying the Highway Safety Manual to Two-Lane Road Curves," Transportation Research Forum, vol. 51, no. 3, pp. 25-38, 2012.

Florida Department of Transportation (FDOT), Transportation Statistics RCI Field Handbook, Tallahassee, FL, 2012.

FDOT, Crashes on Florida's State Highway System (SHS) Roadways, Tallahassee, FL, 2013.

Friedl, M. A., and C. E. Brodley, "Decision Tree Classification of Land Cover from Remotely Sensed Data," Remote Sensing of Environment, vol. 61, no. 3, pp. 399-409, 1997.

Friedman, J. H., "Greedy Function Approximation: A Gradient Boosting Machine," The Annals of Statistics, vol. 29, no. 5, pp. 1189-1232, 2001.

Friedman, J. H., "Stochastic Gradient Boosting," Computational Statistics and Data Analysis, vol. 38, no. 4, pp. 367-378, 2002.

Friedman, J. H., and J. J. Meulman, "Multiple Additive Regression Trees with Application in Epidemiology," Statistics in Medicine, vol. 22, no. 9, pp.1365-1381, 2003. 
Froeschke, J. T., and B. F. Froeschke, "Spatio-Temporal Predictive Model Based on Environmental Factors for Juvenile Spotted Seatrout in Texas Estuaries Using Boosted Regression Trees," Fisheries Research, vol. 111, no. 3, pp. 131-138, 2011.

Gellrich, M., P. Baur, B. H. Robinson, and P. Bebi, "Combining Classification Tree Analyses with Interviews to Study Why Sub-Alpine Grasslands Sometimes Revert to Forest: A Case Study from the Swiss Alps," Agricultural Systems, vol. 96, no. 1-3, pp. 124-138, 2008.

Hadi, M. A., J. Aruldhas, L.-F. Chow, and J. A. Wattleworth, "Estimating Safety Effects of Cross-Section Design for Various Highway Types Using Negative Binomial Regression," Transportation Research Record: Journal of Transportation Research Board, no. 1500, pp. 169-177, 1995.

Hale, R., S. Marshall, K. Jeppe, and V. Pettigrove, "Separating the Effects of Water Physicochemistry and Sediment Contamination on Chironomus Tepperi (Skuse) Survival, Growth and Development: A Boosted Regression Tree Approach," Aquatic Toxicology, vol. 152, pp. 66-73, 2014.

Hand, D. J., H. Mannila, and P. Smyth, Principles of Data Mining. Cambridge, MA: MIT Press, 2001.

Harb, R., X. Yan, E. Radwan, and X. Su, "Exploring Precrash Maneuvers Using Classification Trees and Random Forests," Accident Analysis and Prevention, vol. 41, no. 1, pp. 98-107, 2009.

Hastie, T., R. Tibshirani, and J. H. Friedman, The Elements of Statistical Learning: Data Mining, Inference, and Prediction. New York: Springer, 2009.

Hauer, E., F. M. Council, and Y. Mohammedshah, "Safety Models for Urban Four-Lane Undivided Road Segments," Transportation Research Record: Journal of Transportation Research Board, vol. 1897, pp. 96-105, 2004.

Hilbe, J. M., Negative Binomial Regression. New York: Cambridge University Press, 2011.

Ho, T. K., "Random Decision Forests," in Proceedings of the Third International Conference on Document Analysis and Recognition, vol.1, pp. 278-282, 1995.

Jafari, A., H. Khademi, P. A. Finke, J. Van de Wauw, and S. Ayoubi, "Spatial Prediction of Soil Great Groups by Boosted Regression Trees Using a Limited Point Dataset in an Arid Region, Southeastern Iran," Geoderma, vol. 232-234, pp. 148-163, 2014.

Jalayer, M., and H. Zhou, "A Sensitivity Analysis of Crash Prediction Models Input in the Highway Safety Manual," Paper presented at the ITE Midwestern District Meeting, Milwaukee, WI, 2013. 
Karlaftis, M. G., and I. Golias, "Effects of Road Geometry and Traffic Volumes on Rural Roadway Accident Rates," Accident Analysis and Prevention, vol. 34, no. 3, pp. 357-365, 2002.

Kashani, A. T., and A. S. Mohaymany, "Analysis of the Traffic Injury Severity on TwoLane, Two-Way Rural Roads Based on Classification Tree Models," Safety Science, vol. 49, no. 10, pp. 1314-1320, 2011.

Kweon, Y. J., I. K. Lim, T. L. Turpin, and S. W. Read, "Development and Application of Guidance to Determine the Best Way to Customize the Highway Safety Manual for Virginia," in Proceedings of the 93rd Annual Meeting of the Transportation Research Board, Washington, D.C., 2014.

Lemercier, B., M. Lacoste, M. Loum, and C. Walter, "Extrapolation at Regional Scale of Local Soil Knowledge Using Boosted Classification Trees: A Two-Step Approach," Geoderma, vol. 171-172, pp. 75-84, 2012.

Liaw, A., and M. Wiener, "Classification and Regression by randomForest," $R$ News, vol. 2, no. 3, pp. 18-22, 2002.

Lu, J., "Development of Safety Performance Functions for SafetyAnalyst Applications in Florida," Ph.D. dissertation, Department of Civil and Environmental Engineering, Florida International University, Miami, 2013.

Lubliner, H., and S. D. Schrock, "Calibration of the Highway Safety Manual Prediction Method for Rural Kansas Highways," in Proceedings of the 91st Annual Meeting of the Transportation Research Board (CD-ROM), Washington, D.C., 2012.

Martinelli, F., F. La Torre, and P. Vadi, "Calibration of the Highway Safety Manual's Accident Prediction Model for Italian Secondary Road Network," Transportation Research Record: Journal of Transportation Research Board, no. 2103, pp. 1-9, 2009.

McCullagh, P., and J. A. Nelder, Generalized Linear Models. London: Chapman and Hall, 1989.

Mehta, G., and Y. Lou, "Calibration and Development of Safety Performance Functions for Alabama: Two-Lane, Two-Way Rural Roads and Four-Lane Divided Highways," Transportation Research Record: Journal of Transportation Research Board, no. 2398, pp. 75-82, 2013.

Miaou, S., A. Lu, and H. S. Lum, "Pitfalls of Using $R^{2}$ to Evaluate Goodness of Fit of Accident Prediction Models," Transportation Research Record: Journal of Transportation Research Board, no. 1542, pp. 6-13, 1996.

Milton, J. C., and F. L. Mannering, "The Relationship between Highway Geometrics, Traffic Related Elements, and Motor Vehicle Accidents," Washington State 
Transportation Center (TRAC), Report No. WA-RD 403.1, Final Report Prepared for Washington Department of Transportation, 1996.

Montella, A., L. Colantuoni, and R. Lamberti, "Crash Prediction Models for Rural Motorways," Transportation Research Record: Journal of Transportation Research Board, no. 2083, pp. 180-189, 2008.

Müller, D., P. J. Leitão, and T. Sikor, "Comparing the Determinants of Cropland Abandonment in Albania and Romania Using Boosted Regression Trees," Agricultural Systems, vol. 117, pp. 66-77, 2013.

Neumann, A., J. Holstein, J.-R. Le Gall, and E. Lepage, "Measuring Performance in Health Care: Case-Mix Adjustment by Boosted Decision Trees," Artificial Intelligence in Medicine, vol. 32, no. 2, pp. 97-113, 2004.

Özçift, A., "Random Forests Ensemble Classifier Trained with Data Resampling Strategy to Improve Cardiac Arrhythmia Diagnosis," Computers in Biology and Medicine, vol. 41, no. 5, pp. 265-271, 2011.

Persaud, B., T. Saleem, S. Faisal, C. Lyon, Y. Chen, and A. Sabbaghi, "Adoption of Highway Safety Manual Predictive Methodologies for Canadian Highways," in Proceedings of the Transportation Association of Canada (TAC) Conference and Exhibition, vol. 2, pp. 1037-1053, Fredericton, New Brunswick, 2012.

Poch, M., and F. Mannering, "Negative Binomial Analysis of Intersection-Accident Frequencies," Journal of Transportation Engineering, vol. 122, no. 2, pp. 105-113, 1996.

Puissant, A., S. Rougier, and A. Stumpf, "Object-Oriented Mapping of Urban Trees Using Random Forest Classifiers," International Journal of Applied Earth Observation and Geoinformation, vol. 26, pp. 235-245, 2014.

Qin, X., Z. Chen, and K. Vachal, "Calibration of Highway Safety Manual Predictive Methods for Rural Local Roads," in Proceedings of the Transportation Research Board 93rd Annual Meeting (CD-ROM), Washington, D.C., 2014.

R Core Team, $R$ : A Language and Environment for Statistical Computing. R Foundation for Statistical Computing, Vienna, Austria, 2014. http://www.R-project.org/.

Sawalha, Z., and T. Sayed, "Evaluating Safety of Urban Arterial Roadways," Journal of Transportation Engineering, vol. 127, no. 2, pp. 151-158, 2001.

Shankar, V., F. Mannering, and W. Barfield, "Effect of Roadway Geometrics and Environmental Factors on Rural Freeway Accident Frequencies," Accident Analysis and Prevention, vol. 27, no. 3, pp. 371-389, 1995.

Shin, H., Y.-J. Lee, and S. Dadvar, "The Development of Local Calibration Factors for Implementing the Highway Safety Manual in Maryland," Morgan State University, 
Report No. MD-14-SP209B4J, Final Report Prepared for Maryland State Highway Administration, 2014.

Srinivasan, R., and D. Carter, "Development of Safety Performance Functions for North Carolina," University of North Carolina Highway Safety Research Center, Report No. FHWA/NC/2010-09, Final Report Prepared for North Carolina Department of Transportation, 2009.

Srinivasan, R., D. Carter, and K. Bauer, "Safety Performance Function Decision Guide: SPF Calibration vs. SPF Development," University of North Carolina Highway Safety Research Center, Report No. FHWA-SA-14-004, Final Report Prepared for Federal Highway Administration Office of Safety, 2013.

Srinivasan, S., P. Haas, N. S. Dhakar, R. Hormel, D. Torbic, and H. Douglas, "Development and Calibration of Highway Safety Manual Equations for Florida Conditions," Transportation Research Center, University of Florida, Contract FDOT BDK77 977-06, Final Report Prepared for Florida Department of Transportation Research Office, 2011.

Strobl, C., J. Malley, and G. Tutz, "An Introduction to Recursive Partitioning: Rationale, Application and Characteristics of Classification and Regression Trees, Bagging and Random Forests," Psychological Methods, vol. 14, no. 4, pp. 323-348, 2009.

Sun, C., P. Edara, H. Brown, B. Claros, and K. Nam, "Calibration of the Highway Safety Manual for Missouri," University of Missouri - Columbia, Report No. cmr14-007, Final Report Prepared for Missouri Department of Transportation, 2013.

Sun, X., D. Magri, H. H. Shirazi, S. Gillella, and L. Li, "Application of the Highway Safety Manual : Louisiana Experience with Rural Multilane Highways," in Proceedings of the 90th Annual Meeting of the Transportation Research Board (CD-ROM), Washington, D.C., 2011.

Sun, X., Y. Li, D. Magri, and H. H. Shirazi, "Application of the Highway Safety Manual Draft Chapter: Louisiana Experience," Transportation Research Record: Journal of Transportation Research Board, no. 1950, pp. 55-64, 2006.

Tegge, R. A., J.-H. Jo, and Y. Ouyang, "Development and Application of Safety Performance Functions for Illinois," University of Illinois at Urbana-Champaign, Report No. ICT-10-066, Final Report Prepared for Illinois Center for Transportation Research, 2010.

Trieu, V., S. Park, and J. Mcfadden, "Sensitivity Analysis of Highway Safety Manual Calibration Factors Using Monte-Carlo Simulation," in Proceedings of the 93rd Annual Meeting of the Transportation Research Board (CD-ROM), Washington, D.C., 2014. 
Vincenzi, S., M. Zucchetta, P. Franzoi, M. Pellizzato, F. Pranovi, G. A. De Leo, and P. Torricelli, "Application of a Random Forest Algorithm to Predict Spatial Distribution of the Potential Yield of Ruditapes Philippinarum in the Venice Lagoon, Italy," Ecological Modelling, vol. 222, no. 8, pp. 1471-1478, 2011.

Vogt, A., and J. G. Bared, "Accident Models for Two-Lane Rural Roads: Segments and Intersections," Publication No. FHWA-RD-98-133, Federal Highway Administration, 1998.

Williams, G., Data mining with Rattle and R: The Art of Excavating Data for Knowledge Discovery (Use R!). New York: Springer, 2011.

Yan, X., and E. Radwan, "Analyses of Rear-End Crashes Based on Classification Tree Models," Traffic Injury Prevention, vol. 7, no. 3, pp. 276-282, 2006.

Young, J., and P. Y. Park, "Benefits of Small Municipalities Using Jurisdiction-Specific Safety Performance Functions Rather than the Highway Safety Manual's Calibrated or Uncalibrated Safety Performance Functions," Canadian Journal of Civil Engineering, vol. 40, no. 6, pp. 517-527, 2013.

Zegeer, C.V., J. Hummer, D. Reinfurt, L. Herf, and W. Hunter, "Safety Effect of CrossSection Design for Two-Lane Roads," Goodell-Grivas, Inc., Southfield, MI, Report No. FHWA-RD-87/008, Final Report Prepared for Federal Highway Administration and Transportation Research Board, 1987.

Zhang, M. H, Q. S. Xu, F. Daeyaert, P. J. Lewi, and D. L. Massart, "Application of Boosting to Classification Problems in Chemometrics," Analytica Chimica Acta, vol. 544, no. 1-2, pp. 167-176, 2005. 
APPENDICES 


\section{APPENDIX A}

Descriptive Statistics of Urban and Suburban Arterial Segments and Intersections 
TABLE A-1 Descriptive Statistics for Urban and Suburban Two-lane Undivided Arterials (Number of Segments $=1,791$ )

\begin{tabular}{|c|c|c|c|c|c|}
\hline \multicolumn{2}{|l|}{ Numeric Variable } & \multirow{2}{*}{\begin{tabular}{|c|} 
Minimum \\
750 \\
\end{tabular}} & \multirow{2}{*}{\begin{tabular}{|c|} 
Maximum \\
34,000 \\
\end{tabular}} & \multirow{2}{*}{\begin{tabular}{|c|} 
Average \\
11,227 \\
\end{tabular}} & \multirow{2}{*}{$\begin{array}{c}\text { Standard } \\
\text { Deviation }\end{array}$} \\
\hline \multirow{8}{*}{ AADT (veh/day) } & 2005 & & & & \\
\hline & 2006 & 850 & 36,500 & 11,465 & 4,965 \\
\hline & 2007 & 800 & 34,500 & 11,481 & 5,141 \\
\hline & 2008 & 800 & 29,000 & 11,410 & 5,180 \\
\hline & 2009 & 850 & 30,500 & 10,709 & 5,058 \\
\hline & 2010 & 800 & 27,500 & 10,766 & 4,968 \\
\hline & 2011 & 550 & 27,500 & 10,589 & 4,799 \\
\hline & 2012 & 550 & 28,500 & 10,255 & 4,699 \\
\hline \multicolumn{2}{|l|}{ Segment length (miles) } & 0.04 & 2.00 & 0.34 & 0.35 \\
\hline \multicolumn{2}{|c|}{ Number of major commercial driveways } & 0 & 6 & 0.09 & 0.46 \\
\hline \multicolumn{2}{|c|}{ Number of major residential driveways } & 0 & 26 & 0.12 & 1.07 \\
\hline \multicolumn{2}{|c|}{ Number of major industrial/institutional driveways } & 0 & 9 & 0.11 & 0.53 \\
\hline \multicolumn{2}{|c|}{ Number of minor commercial driveways } & 0 & 43 & 0.71 & 2.74 \\
\hline \multicolumn{2}{|c|}{ Number of minor residential driveways } & 0 & 129 & 4.46 & 10.98 \\
\hline \multicolumn{2}{|c|}{ Number of minor industrial/institutional driveways } & 0 & 27 & 0.74 & 2.52 \\
\hline \multicolumn{2}{|c|}{ Number of other driveways } & 0 & 23 & 0.29 & 1.52 \\
\hline \multicolumn{2}{|c|}{ Roadside fixed object density (objects per mile) } & 0 & 560.98 & 52.25 & 46.50 \\
\hline \multicolumn{2}{|c|}{ Categorical Variable } & \multicolumn{4}{|c|}{ Number of Segments } \\
\hline \multirow{2}{*}{ Speed Category } & $\leq 30 \mathrm{mph}$ & \multicolumn{4}{|c|}{116} \\
\hline & $>30 \mathrm{mph}$ & \multicolumn{4}{|c|}{1,675} \\
\hline \multirow[t]{2}{*}{ Presence of on-street parking } & \begin{tabular}{|l|} 
Yes \\
\end{tabular} & \multicolumn{4}{|c|}{85} \\
\hline & No & \multicolumn{4}{|c|}{1,706} \\
\hline \multirow{2}{*}{ Presence of lighting } & Yes & \multicolumn{4}{|c|}{746} \\
\hline & No & \multicolumn{4}{|c|}{1,045} \\
\hline \multirow{2}{*}{$\begin{array}{l}\text { Presence of automated speed } \\
\text { enforcement }\end{array}$} & Yes & \multicolumn{4}{|c|}{4} \\
\hline & No & \multicolumn{4}{|c|}{1787} \\
\hline
\end{tabular}


TABLE A-2 Descriptive Statistics for Urban and Suburban Three-lane Arterials with a TWLTL (Number of Segments = 359)

\begin{tabular}{|c|c|c|c|c|c|}
\hline \multicolumn{2}{|l|}{ Numeric Variable } & Minimum & Maximum & Average & $\begin{array}{l}\text { Standard } \\
\text { Deviation }\end{array}$ \\
\hline \multirow{8}{*}{ AADT (veh/day) } & 2005 & 3,900 & 34,000 & 15,926 & 5,564 \\
\hline & 2006 & 4,100 & 32,500 & 16,162 & 5,455 \\
\hline & 2007 & 4,100 & 34,500 & 16,303 & 5,928 \\
\hline & 2008 & 4,600 & 30,000 & 15,938 & 5,421 \\
\hline & 2009 & 4,000 & 28,000 & 15,033 & 5,297 \\
\hline & 2010 & 4,000 & 34,500 & 14,577 & 5,086 \\
\hline & 2011 & 3,700 & 27,500 & 14,059 & 4,772 \\
\hline & 2012 & 3,000 & 26,500 & 13,132 & 5,444 \\
\hline \multicolumn{2}{|l|}{ Segment length (miles) } & 0.04 & 1.36 & 0.19 & 0.16 \\
\hline \multicolumn{2}{|c|}{ Number of major commercial driveways } & 0 & 5 & 0.23 & 0.67 \\
\hline \multicolumn{2}{|c|}{ Number of major residential driveways } & 0 & 22 & 0.62 & 1.96 \\
\hline \multicolumn{2}{|c|}{ Number of major industrial/institutional driveways } & 0 & 6 & 0.10 & 0.46 \\
\hline \multicolumn{2}{|c|}{ Number of minor commercial driveways } & 0 & 48 & 2.78 & 5.27 \\
\hline \multicolumn{2}{|c|}{ Number of minor residential driveways } & 0 & 44 & 2.44 & 4.77 \\
\hline \multicolumn{2}{|c|}{ Number of minor industrial/institutional driveways } & 0 & 22 & 0.73 & 1.95 \\
\hline \multicolumn{2}{|c|}{ Number of other driveways } & 0 & 1 & 0.02 & 0.14 \\
\hline \multicolumn{2}{|c|}{ Roadside fixed object density (objects per mile) } & 0 & 250 & 79.58 & 35.56 \\
\hline \multicolumn{2}{|c|}{ Categorical Variable } & \multicolumn{4}{|c|}{ Number of Segments } \\
\hline \multirow{2}{*}{ Speed Category } & $\leq 30 \mathrm{mph}$ & \multicolumn{4}{|c|}{30} \\
\hline & $>30 \mathrm{mph}$ & \multicolumn{4}{|c|}{329} \\
\hline \multirow{2}{*}{ Presence of on-street parking } & \begin{tabular}{|c|} 
Yes \\
\end{tabular} & \multicolumn{4}{|c|}{5} \\
\hline & No & \multicolumn{4}{|c|}{354} \\
\hline \multirow{2}{*}{ Presence of lighting } & Yes & \multicolumn{4}{|c|}{180} \\
\hline & No & \multicolumn{4}{|c|}{179} \\
\hline \multirow{2}{*}{$\begin{array}{l}\text { Presence of automated speed } \\
\text { enforcement }\end{array}$} & Yes & \multicolumn{4}{|c|}{0} \\
\hline & No & \multicolumn{4}{|c|}{359} \\
\hline
\end{tabular}


TABLE A-3 Descriptive Statistics for Urban and Suburban Four-lane Undivided Arterials (Number of Segments $=266$ )

\begin{tabular}{|c|c|c|c|c|c|}
\hline \multicolumn{2}{|l|}{ Numeric Variable } & Minimum & Maximum & Average & $\begin{array}{l}\text { Standard } \\
\text { Deviation } \\
\end{array}$ \\
\hline \multirow{8}{*}{ AADT (veh/day) } & 2005 & 3,600 & 51,500 & 21,538 & 8,984 \\
\hline & 2006 & 3,900 & 48,000 & 21,407 & 8,789 \\
\hline & 2007 & 3,700 & 51,000 & 21,316 & 9,024 \\
\hline & 2008 & 4,300 & 54,500 & 20,540 & 8,845 \\
\hline & 2009 & 3,300 & 46,000 & 19,739 & 8,547 \\
\hline & 2010 & 3,400 & 44,500 & 18,752 & 8,009 \\
\hline & 2011 & 3,900 & 44,500 & 18,555 & 8,226 \\
\hline & 2012 & 3,600 & 47,000 & 18,980 & 8,509 \\
\hline \multicolumn{2}{|c|}{ Segment length (miles) } & 0.04 & 1.87 & 0.20 & 0.18 \\
\hline \multicolumn{2}{|c|}{ Number of major commercial driveways } & 0 & 2 & 0.11 & 0.37 \\
\hline \multicolumn{2}{|c|}{ Number of major residential driveways } & 0 & 8 & 0.12 & 0.65 \\
\hline \multicolumn{2}{|c|}{ Number of major industrial/institutional driveways } & 0 & 4 & 0.15 & 0.52 \\
\hline \multicolumn{2}{|c|}{ Number of minor commercial driveways } & 0 & 28 & 3.17 & 4.58 \\
\hline \multicolumn{2}{|c|}{ Number of minor residential driveways } & 0 & 36 & 2.30 & 5.52 \\
\hline \multicolumn{2}{|c|}{ Number of minor industrial/institutional driveways } & 0 & 40 & 1.79 & 3.66 \\
\hline \multicolumn{2}{|c|}{ Number of other driveways } & 0 & 1 & 0.003 & 0.06 \\
\hline \multicolumn{2}{|c|}{ Roadside fixed object density (objects per mile) } & 0 & 291.67 & 74.67 & 40.22 \\
\hline \multicolumn{2}{|c|}{ Categorical Variable } & \multicolumn{4}{|c|}{ Number of Segments } \\
\hline \multirow{2}{*}{ Speed Category } & $\leq 30 \mathrm{mph}$ & \multicolumn{4}{|c|}{58} \\
\hline & $>30 \mathrm{mph}$ & \multicolumn{4}{|c|}{208} \\
\hline \multirow{2}{*}{ Presence of on-street parking } & Yes & \multicolumn{4}{|c|}{60} \\
\hline & No & \multicolumn{4}{|c|}{206} \\
\hline \multirow{2}{*}{ Presence of lighting } & Yes & \multicolumn{4}{|c|}{243} \\
\hline & No & \multicolumn{4}{|c|}{23} \\
\hline \multirow{2}{*}{$\begin{array}{l}\text { Presence of automated speed } \\
\text { enforcement }\end{array}$} & Yes & \multicolumn{4}{|c|}{0} \\
\hline & No & \multicolumn{4}{|c|}{266} \\
\hline
\end{tabular}


TABLE A-4 Descriptive Statistics for Urban and Suburban Four-lane Divided Arterials (Number of Segments $=4,969$ )

\begin{tabular}{|c|c|c|c|c|c|}
\hline \multicolumn{2}{|l|}{ Numeric Variable } & \multirow{2}{*}{\begin{tabular}{|c|} 
Minimum \\
1,700 \\
\end{tabular}} & \multirow{2}{*}{\begin{tabular}{|c|} 
Maximum \\
88,500 \\
\end{tabular}} & \multirow{2}{*}{\begin{tabular}{|c|} 
Average \\
27,434 \\
\end{tabular}} & \multirow{2}{*}{\begin{tabular}{|c|c|}
$\begin{array}{c}\text { Standard } \\
\text { Deviation }\end{array}$ \\
11,005 \\
\end{tabular}} \\
\hline \multirow{8}{*}{ AADT (veh/day) } & 2005 & & & & \\
\hline & 2006 & 2,100 & 87,500 & 27,899 & 10,966 \\
\hline & 2007 & 2,400 & 96,000 & 28,036 & 10,957 \\
\hline & 2008 & 1,900 & 81,500 & 27,723 & 10,778 \\
\hline & 2009 & 2,500 & 79,500 & 26,490 & 10,541 \\
\hline & 2010 & 1,200 & 88,500 & 25,973 & 10,190 \\
\hline & 2011 & 1,650 & 89,000 & 26,099 & 10,339 \\
\hline & 2012 & 950 & 86,000 & 25,613 & 10,078 \\
\hline \multicolumn{2}{|l|}{ Segment length (miles) } & 0.04 & 2.03 & 0.28 & 0.34 \\
\hline \multicolumn{2}{|c|}{ Number of major commercial driveways } & 0 & 2 & 0.09 & 1.20 \\
\hline \multicolumn{2}{|c|}{ Number of major residential driveways } & 0 & 3 & 0.05 & 0.60 \\
\hline \multicolumn{2}{|c|}{ Number of major industrial/institutional driveways } & 0 & 0 & 0 & 0.22 \\
\hline \multicolumn{2}{|c|}{ Number of minor commercial driveways } & 0 & 19 & 1.86 & 3.78 \\
\hline \multicolumn{2}{|c|}{ Number of minor residential driveways } & 0 & 67 & 1.43 & 2.72 \\
\hline \multicolumn{2}{|c|}{ Number of minor industrial/institutional driveways } & 0 & 0 & 0 & 0.46 \\
\hline \multicolumn{2}{|c|}{ Number of other driveways } & 0 & 0 & 0 & 0.56 \\
\hline \multicolumn{2}{|c|}{ Roadside fixed object density (objects per mile) } & 0 & 586.21 & 144.25 & 59.34 \\
\hline \multicolumn{2}{|c|}{ Categorical Variable } & \multicolumn{4}{|c|}{ Number of Segments } \\
\hline \multirow{2}{*}{ Speed Category } & $\leq 30 \mathrm{mph}$ & \multicolumn{4}{|c|}{97} \\
\hline & $>30 \mathrm{mph}$ & \multicolumn{4}{|c|}{4,872} \\
\hline \multirow{2}{*}{ Presence of on-street parking } & \begin{tabular}{|l|} 
Yes \\
\end{tabular} & \multicolumn{4}{|c|}{74} \\
\hline & No & \multicolumn{4}{|c|}{4,895} \\
\hline \multirow{2}{*}{ Presence of lighting } & Yes & \multicolumn{4}{|c|}{3,699} \\
\hline & No & \multicolumn{4}{|c|}{1,270} \\
\hline \multirow{2}{*}{$\begin{array}{l}\text { Presence of automated speed } \\
\text { enforcement }\end{array}$} & Yes & \multicolumn{4}{|c|}{80} \\
\hline & No & & 4,889 & & \\
\hline & $10 \mathrm{ft}$ & & 205 & & \\
\hline & $20 \mathrm{ft}$ & & 1,941 & & \\
\hline & $30 \mathrm{ft}$ & & 786 & & \\
\hline & $40 \mathrm{ft}$ & & 1,525 & & \\
\hline Median width & $50 \mathrm{ft}$ & & 260 & & \\
\hline snedan widn & $60 \mathrm{ft}$ & & 125 & & \\
\hline & $70 \mathrm{ft}$ & & 51 & & \\
\hline & $80 \mathrm{ft}$ & & 14 & & \\
\hline & $90 \mathrm{ft}$ & & 14 & & \\
\hline & $100 \mathrm{ft}$ & & 46 & & \\
\hline
\end{tabular}


TABLE A-5 Descriptive Statistics for Urban and Suburban Five-lane Arterials with a TWLTL (Number of Segments $=1,084$ )

\begin{tabular}{|c|c|c|c|c|c|}
\hline \multicolumn{2}{|l|}{ Numeric Variable } & Minimum & Maximum & Average & $\begin{array}{l}\text { Standard } \\
\text { Deviation }\end{array}$ \\
\hline \multirow{8}{*}{ AADT (veh/day) } & 2005 & 3,300 & 53,500 & 26,850 & 9,657 \\
\hline & 2006 & 3,800 & 49,500 & 27,123 & 9,513 \\
\hline & 2007 & 4,100 & 56,000 & 27,061 & 9,463 \\
\hline & 2008 & 4,000 & 55,500 & 26,747 & 9,317 \\
\hline & 2009 & 3,700 & 52,000 & 25,548 & 9,249 \\
\hline & 2010 & 4,100 & 55,500 & 24,930 & 8,763 \\
\hline & 2011 & 3,900 & 55,000 & 24,655 & 8,990 \\
\hline & 2012 & 3,800 & 55,000 & 24,128 & 8,682 \\
\hline \multicolumn{2}{|l|}{ Segment length (miles) } & 0.04 & 2.00 & 0.25 & 0.23 \\
\hline \multicolumn{2}{|c|}{ Number of major commercial driveways } & 0 & 13 & 1.10 & 1.85 \\
\hline \multicolumn{2}{|c|}{ Number of major residential driveways } & 0 & 16 & 0.13 & 0.75 \\
\hline \multicolumn{2}{|c|}{ Number of major industrial/institutional driveways } & 0 & 2 & 0.03 & 0.19 \\
\hline \multicolumn{2}{|c|}{ Number of minor commercial driveways } & 0 & 53 & 4.78 & 5.71 \\
\hline \multicolumn{2}{|c|}{ Number of minor residential driveways } & 0 & 43 & 1.36 & 4.09 \\
\hline \multicolumn{2}{|c|}{ Number of minor industrial/institutional driveways } & 0 & 10 & 0.13 & 0.73 \\
\hline \multicolumn{2}{|c|}{ Number of other driveways } & 0 & 1 & 0.00 & 0.03 \\
\hline \multicolumn{2}{|c|}{ Roadside fixed object density (objects per mile) } & 0 & 250 & 75.28 & 31.44 \\
\hline \multicolumn{2}{|c|}{ Curb length with on-street parking (miles) } & 0 & 0 & 0 & 0 \\
\hline \multicolumn{2}{|c|}{ Categorical Variable } & \multicolumn{4}{|c|}{ Number of Segments } \\
\hline \multirow{2}{*}{ Speed Category } & $\leq 30 \mathrm{mph}$ & \multicolumn{4}{|c|}{48} \\
\hline & $>30 \mathrm{mph}$ & \multicolumn{4}{|c|}{1,036} \\
\hline \multirow[t]{2}{*}{ Presence of on-street parking } & Yes & \multicolumn{4}{|c|}{0} \\
\hline & No & \multicolumn{4}{|c|}{1,084} \\
\hline \multirow{2}{*}{ Presence of lighting } & Yes & \multicolumn{4}{|c|}{994} \\
\hline & No & \multicolumn{4}{|c|}{90} \\
\hline \multirow{2}{*}{$\begin{array}{l}\text { Presence of automated speed } \\
\text { enforcement }\end{array}$} & Yes & \multicolumn{4}{|c|}{5} \\
\hline & No & \multicolumn{4}{|c|}{1,079} \\
\hline
\end{tabular}


TABLE A-6 Descriptive Statistics for Urban and Suburban Three-Leg StopControlled Intersections (Number of Intersections = 317)

\begin{tabular}{|c|c|c|c|c|c|}
\hline \multicolumn{2}{|l|}{ Numeric Variable } & Minimum & Maximum & Average & $\begin{array}{l}\text { Standard } \\
\text { Deviation }\end{array}$ \\
\hline \multirow{8}{*}{ Major Road AADT (veh/day) } & 2005 & 500 & 65,000 & 20,690 & 13,354 \\
\hline & 2006 & 500 & 65,500 & 21,073 & 13,364 \\
\hline & 2007 & 500 & 67,000 & 21,181 & 13,432 \\
\hline & 2008 & 550 & 67,000 & 21,024 & 13,041 \\
\hline & 2009 & 550 & 76,500 & 20,094 & 12,909 \\
\hline & 2010 & 250 & 65,500 & 19,516 & 12,351 \\
\hline & 2011 & 550 & 62,000 & 19,474 & 12,710 \\
\hline & 2012 & 900 & 64,500 & 19,061 & 12,768 \\
\hline \multirow{8}{*}{ Minor Road AADT (veh/day) } & 2005 & 100 & 27,500 & 4,091 & 4,033 \\
\hline & 2006 & 100 & 25,000 & 3,885 & 3,663 \\
\hline & 2007 & 100 & 37,500 & 3,972 & 3,916 \\
\hline & 2008 & 100 & 21,500 & 3,932 & 3,427 \\
\hline & 2009 & 100 & 41,500 & 4,001 & 4,002 \\
\hline & 2010 & 100 & 21,500 & 3,801 & 3,274 \\
\hline & 2011 & 80 & 30,000 & 3,663 & 3,340 \\
\hline & 2012 & 120 & 30,000 & 3,309 & 3,329 \\
\hline \multicolumn{2}{|l|}{ Categorical Variable } & \multicolumn{4}{|c|}{ Number of Intersections } \\
\hline \multirow{2}{*}{ Presence of lighting } & Yes & \multicolumn{4}{|c|}{221} \\
\hline & No & \multicolumn{4}{|c|}{96} \\
\hline
\end{tabular}


TABLE A-7 Descriptive Statistics for Urban and Suburban Four-Leg Signalized Intersections (Number of Intersections $=397$ )

\begin{tabular}{|c|c|c|c|c|c|}
\hline \multicolumn{2}{|l|}{ Numeric Variable } & Minimum & Maximum & Average & $\begin{array}{l}\text { Standard } \\
\text { Deviation }\end{array}$ \\
\hline \multirow{8}{*}{ Major Road AADT (veh/day) } & 2005 & 6,300 & 82,000 & 34,341 & 15,005 \\
\hline & 2006 & 6,300 & 79,000 & 34,914 & 14,431 \\
\hline & 2007 & 6,700 & 88,500 & 35,087 & 14,639 \\
\hline & 2008 & 6,364 & 76,500 & 34,394 & 14,355 \\
\hline & 2009 & 1,500 & 77,000 & 33,784 & 14,842 \\
\hline & 2010 & 1,500 & 73,000 & 32,485 & 14,162 \\
\hline & 2011 & 1,500 & 74,500 & 31,991 & 14,275 \\
\hline & 2012 & 1,500 & 76,000 & 31,667 & 14,323 \\
\hline \multirow{8}{*}{ Minor Road AADT (veh/day) } & 2005 & 900 & 57,000 & 19,317 & 12,036 \\
\hline & 2006 & 1,200 & 58,500 & 19,765 & 12,206 \\
\hline & 2007 & 1,200 & 60,500 & 19,903 & 12,214 \\
\hline & 2008 & 1,100 & 56,500 & 19,148 & 11,375 \\
\hline & 2009 & 950 & 57,500 & 19,009 & 11,719 \\
\hline & 2010 & 1,100 & 59,500 & 18,689 & 11,601 \\
\hline & 2011 & 1,100 & 57,000 & 18,792 & 12,025 \\
\hline & 2012 & 950 & 55,000 & 18,272 & 11,550 \\
\hline \multicolumn{2}{|l|}{ Categorical Variable } & \multicolumn{4}{|c|}{ Number of Intersections } \\
\hline \multirow{2}{*}{ Presence of lighting } & Yes & \multicolumn{4}{|c|}{370} \\
\hline & No & \multicolumn{4}{|c|}{27} \\
\hline \multirow{2}{*}{ Presence of red-light camera } & Yes & \multicolumn{4}{|c|}{97} \\
\hline & No & \multicolumn{4}{|c|}{300} \\
\hline \multirow{2}{*}{ Presence of schools within $1,000 \mathrm{ft}$} & Yes & \multicolumn{4}{|c|}{67} \\
\hline & No & \multicolumn{4}{|c|}{330} \\
\hline \multirow{3}{*}{ Number of bus stops within $1,000 \mathrm{ft}$} & 0 & \multicolumn{4}{|c|}{127} \\
\hline & $1-2$ & \multicolumn{4}{|c|}{72} \\
\hline & $\geq 3$ & \multicolumn{4}{|c|}{198} \\
\hline \multirow{3}{*}{$\begin{array}{l}\text { Number of alcohol sales } \\
\text { establishments within } 1,000 \mathrm{ft}\end{array}$} & 0 & \multicolumn{4}{|c|}{40} \\
\hline & $1-8$ & \multicolumn{4}{|c|}{355} \\
\hline & $\geq 3$ & \multicolumn{4}{|c|}{2} \\
\hline
\end{tabular}


APPENDIX B

Regression Coefficients and Crash Modification Factors (CMFs) 
TABLE B-1 Coefficients of SPF for Multiple-Vehicle Non-Driveway Crashes on Urban and Suburban Arterial Segments

\begin{tabular}{|l|c|c|c|}
\hline Segment Type & $\boldsymbol{\alpha}_{\mathbf{0}}$ & $\boldsymbol{\alpha}_{\mathbf{1}}$ & $\begin{array}{c}\text { Overdispersion } \\
\text { Parameter }\end{array}$ \\
\hline Two-lane undivided & -15.22 & 1.68 & 0.84 \\
\hline Three-lane with a TWLTL & -12.40 & 1.41 & 0.66 \\
\hline Four-lane undivided & -11.63 & 1.33 & 1.01 \\
\hline Four-lane divided & -12.34 & 1.36 & 1.32 \\
\hline Five-lane with a TWLTL & -9.70 & 1.17 & 0.81 \\
\hline
\end{tabular}

TABLE B-2 Coefficients of SPF for Single-Vehicle Crashes on Urban and Suburban Arterial Segments

\begin{tabular}{|l|c|c|c|}
\hline Segment Type & $\boldsymbol{\beta}_{\mathbf{0}}$ & $\boldsymbol{\beta}_{\mathbf{1}}$ & $\begin{array}{c}\text { Overdispersion } \\
\text { Parameter }\end{array}$ \\
\hline Two-lane undivided & -5.47 & 0.56 & 0.81 \\
\hline Three-lane with a TWLTL & -5.74 & 0.54 & 1.37 \\
\hline Four-lane undivided & -7.99 & 0.81 & 0.91 \\
\hline Four-lane divided & -5.05 & 0.47 & 0.86 \\
\hline Five-lane with a TWLTL & -4.82 & 0.54 & 0.52 \\
\hline
\end{tabular}

Table B-3 Coefficients of SPF for Multiple-Vehicle Driveway-Related Crashes on Urban and Suburban Arterial Segments

\begin{tabular}{|l|c|c|c|c|c|}
\hline Driveway Type (j) & $\begin{array}{c}\text { Two-Lane } \\
\text { Undivided }\end{array}$ & $\begin{array}{c}\text { Four-Lane } \\
\text { Undivided }\end{array}$ & $\begin{array}{c}\text { Four-Lane } \\
\text { Divided }\end{array}$ & $\begin{array}{c}\text { Three-Lane } \\
\text { with a } \\
\text { TWLTL }\end{array}$ & $\begin{array}{c}\text { Five-Lane } \\
\text { with a } \\
\text { TWLTL }\end{array}$ \\
\hline \multicolumn{7}{|c|}{ Number of Driveway-Related Crashes per Driveway per Year $\left(\boldsymbol{N}_{\boldsymbol{j}}\right.$ ) } \\
\hline Major commercial & 0.158 & 0.182 & 0.033 & 0.102 & 0.165 \\
\hline Minor commercial & 0.050 & 0.058 & 0.011 & 0.032 & 0.053 \\
\hline Major industrial/institutional & 0.172 & 0.198 & 0.036 & 0.110 & 0.181 \\
\hline Minor industrial/institutional & 0.023 & 0.026 & 0.005 & 0.015 & 0.024 \\
\hline Major residential & 0.083 & 0.096 & 0.018 & 0.053 & 0.087 \\
\hline Minor residential & 0.016 & 0.018 & 0.003 & 0.010 & 0.016 \\
\hline Other & 0.025 & 0.029 & 0.005 & 0.016 & 0.027 \\
\hline \multicolumn{7}{|c|}{ AADT $(\boldsymbol{t})$} & 1.172 & 1.106 & 1.000 & 1.172 \\
\hline All driveways & 1.000 & Overdispersion Parameter & 1.10 & 0.10 \\
\hline
\end{tabular}


TABLE B-4 On-Street Parking Factor $\left(f_{p k}\right)$ Used in Determining CMF for On-Street Parking

\begin{tabular}{|l|c|c|c|c|}
\hline \multirow{2}{*}{ Segment Type } & \multicolumn{4}{|c|}{ Type of Parking and Land Use } \\
\cline { 2 - 5 } & \multicolumn{2}{|c|}{ Parallel Parking } & \multicolumn{2}{c|}{ Angle Parking } \\
\cline { 2 - 5 } & $\begin{array}{c}\text { Residential/ } \\
\text { Other }\end{array}$ & $\begin{array}{c}\text { Commercial } \\
\text { or Industrial/ } \\
\text { Institutional }\end{array}$ & $\begin{array}{c}\text { Residential/ } \\
\text { Other }\end{array}$ & $\begin{array}{c}\text { Commercial } \\
\text { or Industrial/ } \\
\text { Institutional }\end{array}$ \\
\hline Two-lane undivided & 1.465 & 2.074 & 3.428 & 4.853 \\
\hline Three-lane with a TWLTL & 1.465 & 2.074 & 3.428 & 4.853 \\
\hline Four-lane undivided & 1.100 & 1.709 & 2.574 & 3.999 \\
\hline Four-lane divided & 1.100 & 1.709 & 2.574 & 3.999 \\
\hline Five-lane with a TWLTL & 1.100 & 1.709 & 2.574 & 3.999 \\
\hline
\end{tabular}

TABLE B-5 Fixed-Object Offset Factor $\left(f_{\text {offset }}\right)$ Used in Determining CMF for Roadside Fixed Objects

\begin{tabular}{|c|c|}
\hline $\begin{array}{c}\text { Offset to Fixed Objects } \\
\text { (ft) }\end{array}$ & $\begin{array}{c}\text { Fixed-Object Offset Factor } \\
\left(\boldsymbol{f}_{\text {offset }}\right)\end{array}$ \\
\hline 2 & 0.232 \\
\hline 5 & 0.133 \\
\hline 10 & 0.087 \\
\hline 15 & 0.068 \\
\hline 20 & 0.057 \\
\hline 25 & 0.049 \\
\hline 30 & 0.044 \\
\hline
\end{tabular}

TABLE B-6 CMFs for Median Width on Urban and Suburban Four-Lane Divided Arterials

\begin{tabular}{|c|c|}
\hline $\begin{array}{c}\text { Median Width } \\
\text { (ft) }\end{array}$ & CMF \\
\hline 10 & 1.01 \\
\hline 15 & 1.00 \\
\hline 20 & 0.99 \\
\hline 30 & 0.98 \\
\hline 40 & 0.97 \\
\hline 50 & 0.96 \\
\hline 60 & 0.95 \\
\hline 70 & 0.94 \\
\hline 80 & 0.93 \\
\hline 90 & 0.93 \\
\hline 100 & 0.92 \\
\hline
\end{tabular}


TABLE B-7 Coefficients of SPF for Multiple-Vehicle Crashes on Urban and Suburban Intersections

\begin{tabular}{|l|c|c|c|c|}
\hline Intersection Type & $\gamma_{\mathbf{0}}$ & $\gamma_{\mathbf{1}}$ & $\boldsymbol{\gamma}_{\mathbf{2}}$ & $\begin{array}{c}\text { Overdispersion } \\
\text { Parameter }\end{array}$ \\
\hline Three-leg stop-controlled & -13.36 & 1.11 & 0.41 & 0.80 \\
\hline Four-leg signalized & -10.99 & 1.07 & 0.23 & 0.39 \\
\hline
\end{tabular}

TABLE B-8 Coefficients of SPF for Single-Vehicle Crashes on Urban and Suburban Intersections

\begin{tabular}{|l|c|c|c|c|}
\hline Intersection Type & $\boldsymbol{\delta}_{\mathbf{0}}$ & $\boldsymbol{\delta}_{\mathbf{1}}$ & $\boldsymbol{\delta}_{\mathbf{2}}$ & $\begin{array}{c}\text { Overdispersion } \\
\text { Parameter }\end{array}$ \\
\hline Three-leg stop-controlled & -6.81 & 0.16 & 0.51 & 1.14 \\
\hline Four-leg signalized & -10.21 & 0.68 & 0.27 & 0.36 \\
\hline
\end{tabular}

TABLE B-9 Coefficients of SPF for Vehicle-Pedestrian Crashes on Urban and Suburban Intersections

\begin{tabular}{|l|c|c|c|c|c|c|}
\hline Intersection Type & $\boldsymbol{a}$ & $\boldsymbol{b}$ & $\boldsymbol{c}$ & $\boldsymbol{d}$ & $\boldsymbol{e}$ & $\begin{array}{c}\text { Overdispersion } \\
\text { Parameter }\end{array}$ \\
\hline Three-leg signalized & -6.60 & 0.05 & 0.24 & 0.41 & 0.09 & 0.52 \\
\hline Four-leg signalized & -9.53 & 0.40 & 0.26 & 0.45 & 0.04 & 0.24 \\
\hline
\end{tabular}


APPENDIX C

Florida-Specific Crash Proportions 
TABLE C-1 Proportion of Total Crashes That Occurred at Night on Unlighted Segments $\left(p_{n r}\right)$ of Urban and Suburban Arterials

\begin{tabular}{|l|c|c|c|c|c|c|c|c|c|}
\hline \multirow{2}{*}{ Segment Type } & \multicolumn{6}{|c|}{ Florida-Specific Values by Years } & $\begin{array}{c}\text { HSM- } \\
\text { Default } \\
\text { Values }\end{array}$ \\
\cline { 2 - 10 } & $\mathbf{2 0 0 5}$ & $\mathbf{2 0 0 6}$ & $\mathbf{2 0 0 7}$ & $\mathbf{2 0 0 8}$ & $\mathbf{2 0 0 9}$ & $\mathbf{2 0 1 0}$ & $\mathbf{2 0 1 1}$ & $\mathbf{2 0 1 2}$ & $\begin{array}{c}\mathbf{2 0 1} \\
\text { Value }\end{array}$ \\
\hline Two-lane undivided & 0.215 & 0.237 & 0.243 & 0.240 & 0.250 & 0.233 & 0.224 & 0.248 & 0.316 \\
\hline $\begin{array}{l}\text { Three-lane with a } \\
\text { TWLTL }\end{array}$ & 0.124 & 0.100 & 0.123 & 0.144 & 0.112 & 0.228 & 0.104 & 0.126 & 0.304 \\
\hline Four-lane undivided & 0.111 & 0.114 & 0.179 & 0.067 & 0.027 & 0.091 & 0.036 & 0.000 & 0.365 \\
\hline Four-lane divided & 0.193 & 0.187 & 0.224 & 0.196 & 0.197 & 0.179 & 0.186 & 0.178 & 0.410 \\
\hline $\begin{array}{l}\text { Five-lane with a } \\
\text { TWLTL }\end{array}$ & 0.123 & 0.084 & 0.073 & 0.137 & 0.113 & 0.147 & 0.085 & 0.061 & 0.274 \\
\hline
\end{tabular}

TABLE C-2 Proportion of Total Nighttime Crashes That Involved a Fatality or Injury on Unlighted Segments $\left(p_{i n r}\right)$ of Urban and Suburban Arterials

\begin{tabular}{|l|c|c|c|c|c|c|c|c|c|}
\hline \multirow{2}{*}{ Segment Type } & \multicolumn{6}{|c|}{ Florida-Specific Values by Years } & $\begin{array}{c}\text { HSM- } \\
\text { Default } \\
\text { Values }\end{array}$ \\
\cline { 2 - 10 } & $\mathbf{2 0 0 5}$ & $\mathbf{2 0 0 6}$ & $\mathbf{2 0 0 7}$ & $\mathbf{2 0 0 8}$ & $\mathbf{2 0 0 9}$ & $\mathbf{2 0 1 0}$ & $\mathbf{2 0 1 1}$ & $\mathbf{2 0 1 2}$ & $\begin{array}{c}\mathbf{2 0 1} \\
\text { Value }\end{array}$ \\
\hline Two-lane undivided & 0.578 & 0.561 & 0.650 & 0.658 & 0.612 & 0.642 & 0.543 & 0.558 & 0.424 \\
\hline $\begin{array}{l}\text { Three-lane with a } \\
\text { TWLTL }\end{array}$ & 0.467 & 0.800 & 0.714 & 0.688 & 0.667 & 0.619 & 0.636 & 0.667 & 0.429 \\
\hline Four-lane undivided & 1.000 & 0.750 & 0.600 & 0.000 & 1.000 & 0.750 & 1.000 & 0.000 & 0.517 \\
\hline Four-lane divided & 0.584 & 0.603 & 0.587 & 0.615 & 0.575 & 0.557 & 0.542 & 0.536 & 0.364 \\
\hline $\begin{array}{l}\text { Five-lane with a } \\
\text { TWLTL }\end{array}$ & 0.684 & 0.500 & 0.700 & 0.632 & 0.692 & 0.647 & 0.636 & 0.667 & 0.432 \\
\hline
\end{tabular}

TABLE C-3 Proportion of Total Nighttime Crashes That Involved PDO on Unlighted Segments $\left(p_{p n r}\right)$ of Urban and Suburban Arterials

\begin{tabular}{|l|c|c|c|c|c|c|c|c|c|}
\hline \multirow{2}{*}{ Segment Type } & \multicolumn{7}{|c|}{ Florida-Specific Values by Years } & $\begin{array}{c}\text { HSM- } \\
\text { Default } \\
\text { Values }\end{array}$ \\
\cline { 2 - 11 } & $\mathbf{2 0 0 5}$ & $\mathbf{2 0 0 6}$ & $\mathbf{2 0 0 7}$ & $\mathbf{2 0 0 8}$ & $\mathbf{2 0 0 9}$ & $\mathbf{2 0 1 0}$ & $\mathbf{2 0 1 1}$ & $\mathbf{2 0 1 2}$ & $\begin{array}{c}\text { Value } \\
\text { Two-lane undivided }\end{array}$ \\
\hline & 0.422 & 0.439 & 0.350 & 0.342 & 0.388 & 0.358 & 0.457 & 0.442 & 0.576 \\
\hline $\begin{array}{l}\text { Three-lane with a } \\
\text { TWLTL }\end{array}$ & 0.533 & 0.200 & 0.286 & 0.313 & 0.333 & 0.381 & 0.364 & 0.333 & 0.571 \\
\hline Four-lane undivided & 0.000 & 0.250 & 0.400 & 1.000 & 0.000 & 0.000 & 0.000 & 0.000 & 0.483 \\
\hline Four-lane divided & 0.416 & 0.397 & 0.413 & 0.385 & 0.425 & 0.443 & 0.458 & 0.464 & 0.636 \\
\hline $\begin{array}{l}\text { Five-lane with a } \\
\text { TWLTL }\end{array}$ & 0.316 & 0.500 & 0.300 & 0.368 & 0.308 & 0.353 & 0.364 & 0.333 & 0.568 \\
\hline
\end{tabular}


TABLE C-4 Proportion of Total Crashes That Occurred at Night on Unlighted Intersections $\left(P_{n i}\right)$ on Urban and Suburban Arterials

\begin{tabular}{|l|c|c|c|c|c|c|c|c|c|}
\hline \multirow{2}{*}{ Intersection Type } & \multicolumn{6}{|c|}{ Florida-Specific Values by Years } & HSM- \\
\cline { 2 - 10 } & $\mathbf{2 0 0 5}$ & $\mathbf{2 0 0 6}$ & $\mathbf{2 0 0 7}$ & $\mathbf{2 0 0 8}$ & $\mathbf{2 0 0 9}$ & $\mathbf{2 0 1 0}$ & $\mathbf{2 0 1 1}$ & $\mathbf{2 0 1 2}$ & $\begin{array}{c}\text { Default } \\
\text { Values }\end{array}$ \\
\hline Three-leg stop-controlled & 0.137 & 0.208 & 0.176 & 0.150 & 0.118 & 0.179 & 0.171 & 0.134 & 0.238 \\
\hline Four-leg signalized & 0.105 & 0.082 & 0.095 & 0.095 & 0.068 & 0.116 & 0.100 & 0.084 & 0.235 \\
\hline
\end{tabular}

TABLE C-5 Bicycle Crash Adjustment Factors for Intersections $\left(f_{\text {bike }}\right)$ on Urban and Suburban Arterials

\begin{tabular}{|l|c|c|c|c|c|c|c|c|c|}
\hline \multirow{2}{*}{ Intersection Type } & \multicolumn{6}{|c|}{ Florida-Specific Values by Years } & HSM- \\
\cline { 2 - 10 } & $\mathbf{2 0 0 5}$ & $\mathbf{2 0 0 6}$ & $\mathbf{2 0 0 7}$ & $\mathbf{2 0 0 8}$ & $\mathbf{2 0 0 9}$ & $\mathbf{2 0 1 0}$ & $\mathbf{2 0 1 1}$ & $\mathbf{2 0 1 2}$ & $\begin{array}{c}\text { Default } \\
\text { Values }\end{array}$ \\
\hline Three-leg stop-controlled & 0.017 & 0.018 & 0.016 & 0.023 & 0.028 & 0.030 & 0.011 & 0.015 & 0.016 \\
\hline Four-leg signalized & 0.008 & 0.012 & 0.009 & 0.012 & 0.011 & 0.011 & 0.014 & 0.012 & 0.015 \\
\hline
\end{tabular}

TABLE C-6 Pedestrian Crash Adjustment Factors for Intersections $\left(f_{\text {bikei }}\right)$ on Urban and Suburban Arterials

\begin{tabular}{|l|c|c|c|c|c|c|c|c|c|}
\hline \multirow{2}{*}{ Intersection Type } & \multicolumn{6}{|c|}{ Florida-Specific Values by Years } & HSM- \\
\cline { 2 - 10 } & $\mathbf{2 0 0 5}$ & $\mathbf{2 0 0 6}$ & $\mathbf{2 0 0 7}$ & $\mathbf{2 0 0 8}$ & $\mathbf{2 0 0 9}$ & $\mathbf{2 0 1 0}$ & $\mathbf{2 0 1 1}$ & $\mathbf{2 0 1 2}$ & $\begin{array}{c}\text { Default } \\
\text { Values }\end{array}$ \\
\hline Three-leg stop-controlled & 0.030 & 0.018 & 0.020 & 0.010 & 0.020 & 0.009 & 0.010 & 0.012 & 0.021 \\
\hline
\end{tabular}

TABLE C-7 Proportion of Specific Crashes on Urban and Suburban Four-Leg Signalized Intersections

\begin{tabular}{|l|l|l|l|l|l|l|l|l|}
\hline Crash Type & $\mathbf{2 0 0 5}$ & $\mathbf{2 0 0 6}$ & $\mathbf{2 0 0 7}$ & $\mathbf{2 0 0 8}$ & $\mathbf{2 0 0 9}$ & $\mathbf{2 0 1 0}$ & $\mathbf{2 0 1 1}$ & $\mathbf{2 0 1 2}$ \\
\hline $\begin{array}{l}\text { Multiple-Vehicle Right-Angle } \\
\left(p_{r a}\right)\end{array}$ & 0.293 & 0.285 & 0.286 & 0.291 & 0.273 & 0.256 & 0.261 & 0.264 \\
\hline Multiple-Vehicle Rear-End $\left(p_{r e}\right)$ & 0.408 & 0.406 & 0.418 & 0.423 & 0.458 & 0.465 & 0.503 & 0.514 \\
\hline
\end{tabular}


VITA

\section{DIBAKAR SAHA}

\section{EDUCATION}

2003 - 2008 B.S., Civil Engineering

Bangladesh University of Engineering and Technology, Dhaka, Bangladesh

2008 - 2010 M.S., Civil Engineering (Transportation)

Bangladesh University of Engineering and Technology, Dhaka, Bangladesh

2010 - 2014 Graduate Research/Teaching Assistant

Department of Civil and Environmental Engineering

Florida International University, Miami, Florida

2012 - 2014 Doctoral Candidate

Department of Civil and Environmental Engineering

Florida International University, Miami, Florida

\section{PUBLICATIONS AND PRESENTATIONS}

1. Saha, D., P. Alluri, and A. Gan, "Prioritizing Highway Safety Manual's Crash Prediction Variables Using Boosted Regression Trees," Accident Analysis and Prevention (second review).

2. Alluri, P., D. Saha, and A. Gan, "Minimum Sample Sizes for Estimating Reliable Highway Safety Manual (HSM) Calibration Factors," Journal of Transportation Safety and Security (in press).

3. Suksawang, N., P. Alluri, A. Gan, K. Meneses, F. Cevallos, K. Haleem, and D. Saha, "Use of Movable Bus Stop Loading Pads: Feasibility and Design Alternatives", Journal of Public Transportation, Vol. 17, No. 4, pp. 14-30, 2014.

4. Alluri, P., D. Saha, and A. Gan, "Prioritization of Highway Safety Manual (HSM) Data Variables Using Random Forest Algorithm," in Proceedings of the 94th Annual Meeting of the Transportation Research Board of the National Academies, Paper No. 15-2257, Washington, D.C., January 2015.

5. Haleem, K., A. Gan, P. Alluri, and D. Saha, "Identifying Traffic Safety Practices and Needs of Local Transportation and Law Enforcement Agencies," Journal of the Transportation Research Forum, Vol. 53, No. 1, pp. 83-99, 2014.

6. Alluri, P., D. Saha, K. Liu, and A. Gan, "Improved Processes for Meeting the Data Requirements for Implementing the Highway Safety Manual (HSM) and 
SafetyAnalyst in Florida," Final Research Report Prepared for Florida Department of Transportation, March 2014.

7. Saha, D., P. Alluri, K. Haleem, and A. Gan, "Evaluation of Hybrid Static-Dynamic Sign Designs: A Focus Group Study," in Proceedings of the 93rd Annual Meeting of the Transportation Research Board of the National Academies, Paper No. 14-5616, Washington, D.C., January 2014.

8. Haleem, K., A. Gan, P. Alluri, and D. Saha, "Identification of Safety Practices, Issues, and Needs of Local Transportation and Law Enforcement Agencies," in Proceedings of the 93rd Annual Meeting of the Transportation Research Board of the National Academies, Paper No. 14-3440, Washington, D.C., January 2014.

9. Suksawang, N., P. Alluri, A. Gan, K. Meneses, F. Cevallos, K. Haleem, and D. Saha, "Feasibility and Design of Movable Bus Stop Boarding and Alighting Pads," in Proceedings of the 93rd Annual Meeting of the Transportation Research Board of the National Academies, Paper No. 14-4448, Washington, D.C., January 2014.

10. Alluri, P., K. Haleem, A. Gan, M. Lavasani, and D. Saha, "Comprehensive Study to Reduce Pedestrian Crashes in Florida," Final Research Report Prepared for Florida Department of Transportation, December 2013.

11. Suksawang, N., A. Gan, P. Alluri, K. Haleem, K. Meneses, F. Cevallos, and D. Saha, "Analysis of Movable Bus Stop Boarding and Alighting Areas," Final Research Report Prepared for Florida Department of Transportation, May 2013.

12. Saha, D., A. Gan, K. Haleem, P. Alluri, and F. H. Delgado, "Applications of Hybrid Static-Dynamic Signs for Active Traffic Management: A Worldwide State-of-thePractice Review," in Proceedings of the 92nd Annual Meeting of the Transportation Research Board of the National Academies, Paper No. 13-4307, Washington, D.C., January 2013.

13. Gan, A., D. Saha, K. Haleem, P. Alluri, and D. McCarthy, "Best Practices in the Use of Hybrid Static-Dynamic Signs," Final Research Report Prepared for Florida Department of Transportation, December 2012.

14. Saha, D., A. Gan, K. Haleem, and F. H. Delgado, "Use of Hybrid Static-Dynamic Signs: A Critical State-of-the-Practice Review," in Proceedings of the 53rd Annual Transportation Research Forum (online), Tampa, Florida, March 2012.

(URL: $\underline{\text { http://www.trforum.org/forum/proceedings.php?year=2012) }}$

15. Gan, A., K. Haleem, P. Alluri, and D. Saha, "Standardization of Crash Analysis in Florida," Final Research Report Prepared for Florida Department of Transportation, February 2012. 